\title{
INDEX OF FIGURED PLANT MEGAFOSSILS PERMIAN 1976-1980
}

\author{
M. BOERSMA ${ }^{1}$ and L.M. BROEKMEYER ${ }^{2}$ \\ ${ }^{1}$ Laboratory of Palaeobotany and Palynology, State University of Utrecht, Heidelberglaan 2, 3584 CS Utrecht \\ (The Netherlands) \\ ${ }^{2}$ Institute for the History of Science, State University of Utrecht, Janskerkhof 30,3512 BN Utrecht (The Netherlands)
}

(Received September 1, 1986; accepted December 16, 1986)

\section{Introduction}

When we started the IFPM Permian 19761980 our files held information on 545 possibly useful publications. This paper is the result of the investigation of 463 of them, i.e. $85 \%$. The remaining 82 papers, not available to us, are obviously hard to get at, since we did not get the expected response on a number of letters to colleagues abroad. On the other hand we are proud to present an extensive list of contributors to the IFPM. We would be very pleased to receive any additional information with regard to the inevitable mistakes and omissions.

The remarks made in this paper do not represent our personal opinion: they are meant to draw attention to conflicting opinions in the literature studied. A marginal "+" has the same function with regard to nomenclature.

From this paper onwards we will refrain from extensive explanatory notes to the text: we assume that the users are familiar with the system used. New users are requested to consult the previous issues. For reference works consulted the reader is referred to IFPM Triassic 1976-1980.

This paper holds information on 398 genera and 1128 species, among which 58 new genera, 337 new species and 3 new taxa of infraspecific rank. The plant megafossils have been described and figured from 37 countries (120 geographical units). The number of useful papers amounts to 259 .

\section{ABBREVIATIONS OF STRATIGRAPHICAL UNITS}

D

C

$\mathrm{P}$

G

$\mathrm{K}$

$\mathrm{T}$

L

$\mathrm{M}$

$\mathrm{U}$

$\begin{array}{ll}\text { UPBara } & \text { Barakar } \\ \text { UPKamt } & \text { Kamthi } \\ \text { UPKarh } & \text { Karharbari } \\ \text { UPKaza } & \text { Kazanian } \\ \text { UPKupf } & \text { Kupferschiefer } \\ \text { UPRani } & \text { Raniganj } \\ \text { UPTata } & \text { Tatarian } \\ \text { UPThur } & \text { Thuringian } \\ \text { UPZech } & \text { Zechstein } \\ \text { LPArti } & \text { Artinskian } \\ \text { LPAutu } & \text { Autunian } \\ \text { LPBara } & \text { Barakar } \\ \text { LPKung } & \text { Kungurian } \\ \text { LPRotl } & \text { Rotliegendes } \\ \text { LPSakm } & \text { Sakmarian } \\ \text { LPSaxo } & \text { Saxonian } \\ \text { LPUfim } & \text { Ufimian } \\ \text { LPWolf } & \text { Wolfcampian } \\ \text { LGBara } & \text { Barakar } \\ \text { LGBarM } & \text { Barren Measures }\end{array}$

Devonian

Carboniferous

Permian

Gondwana

Karroo

Triassic
Lower (Permian, Gondwana, etc.)

Middle (Permian, Gondwana, etc.)

Upper (Permian, Gondwana, etc.)

$0034-6667 / 87 / \$ 03.50$

(C) 1987 Elsevier Science Publishers B.V. 


\section{LGKamt Kamthi \\ LGKarh Karharbari \\ LGRani Raniganj \\ LGTalc Talchir \\ LKEcca Ecca}

\section{CONTRIBUTORS TO THE IFPM PERMIAN 1976-1980}

Anderson, J. (Pretoria)

Archangelsky, S. (Buenos Aires)

Asama, K. (Tokyo)

Banks, H.P. (Ithaca, N.Y.)

Barthel, M. (Berlin)

Bernardes de Oliveira-Babinski, M.E.C. (São Paulo)

Bohlin, B. (Uppsala)

Broutin, J. (Paris)

Cazzulo-Klepzig, M. (Porto Allegre)

Chaloner, W.G. (London)

Chandra, S. (Lucknow)

Daber, R. (Berlin)

Durante, M. (Moscow)

Fefilova, L.A. (Syktyvkar, U.S.S.R.)

Feng, S.-n. (Nanjing)

Fritz, A. (Klagenfurt)

Galtier, J. (Montpellier)

Gillespie, W.H. (Charleston, W.Va.)
Huang, B.-h. (Beiling)

Kerp, J.H.F. (Utrecht, Netherlands)

Kimura, T. (Tokyo)

Koeniguer, J.C. (Paris)

Krasilov, V.A. (Vladivostok)

Lacey, W.S. (Bangor, Wales)

Li, X.-x. (Nanjing)

Lyons, P.C. (Reston, W.Va.)

Maithy, P.K. (Lucknow)

Mamay, S.H. (Washington, D.C.)

Marguerier, J. (Paris)

Meien, S.V. (Moscow)

Pfefferkorn, H.W. (Heidelberg)

Prasad, M.N.V. (Shillong, India)

Remy, W. (Münster, G.F.R.)

Retallack, G. (Bloomington, Ind.)

Rigby, J.F. (Brisbane)

Rösler, O. (São Paulo)

Scheven, J. (Ennepetal, G.F.R.)

Shute, C.H. (London)

Storch, D. (Jena)

Taylor, T.N. (Columbus, Ohio)

Tenchov, Y.G. (Sofia)

Tian, B.-l. (Beijing)

Van Amerom, H.W.J. (Heerlen, Netherlands)

Vogellehner, D. (Freiburg i. Br., G.F.R.)

Wang, R.-n. (Handan, Hebei)

Zhao, X.-h. (Nanjing)

Zimina, V.G. (Vladivostok)

\section{INDEX OF FIGURED PLANT MEGAFOSSILS}

\section{Abietopitys Kräusel 1928}

A. perforata (Gothan 1908) Kräusel 1928

1978 Mussa (a), fig. 2A. B

(scheme from Kräusel)

\section{Abrotopteris Mo 1980}

A. guizhouensis (Gu et Zhi 1974) Mo 1980

1980 Zhao et al., pl.19. 1 7.8(?)

China (Guizhou/Yunnan)

UP

Acitheca Schimper 1891

A. sp.

1977 Remy et Remy, fig.56B

A. cupressoides Gu et Zhi 1974

1978 Zhang, pl.155, 4(?)

A. polymorpha (Brongniart 1834) Schimper 1891

1977 Remy et Remy, fig.123B (ex gr.)

A. unifurcata Yang et Chen ex Zhang 1978

1978 Zhang, pl.155, 7, 8

$\begin{array}{ll}\text { (scheme) } & \text { (C P) } \\ \text { China (Guizhou) } & \text { UP } \\ \text { (scheme) } & \text { (C.P) } \\ \text { China (Guizhou) } & \text { UP }\end{array}$




\section{Alethopteris Sternberg 1825}

A. sp.

1976 Balasundaram et al., pl.22, 5

1979 Yuasa, fig. 3,9

A. firmata Zhang 1978

1978 Zhang, pl.158, 3

A. grandini (Brongniart 1833) Goeppert 1836

1976 Barthel (a), pl.2, 5, 6

1978 Gillespie et al., pl.35, 7

1978 Gillespie et al., pl.35, 9

A. kaipingiana Stockmans et Mathieu 1939

1976 Huang, pl.217, 4-5 (cf.)

A. norinii Halle 1927

1976 Asama, fig.1 (10)

1976 Huang, pl.221, 4, 5

1979 Asama, figs.3 (10), 17C

1980 Asama, fig.5

A. pennsylvanica Lesquereux 1858

1978 Gillespie et al., pl.35, 2, 5

A. schneideri Sterzel 1918

1976 Barthel (a), fig.1; pl.1, 1-13; pl.2, 7

1976 Barthel (a), pl.2, 1-4

1976 Barthel (a), pl.2, 5, 6

1976 Barthel (b), pl.4, 8

1976 Barthel et al., figs.13, 14; pl.36, 1-5, 6-10 (cuti); pl.37, 1-10;

pl.39, 9 (cuti)

1976 Barthel et al., fig.15

A. serlii (Brongniart 1828) Goeppert 1836

1978 Gillespie et al., pl.35, 4, 6

1978 Gillespie et al., pl.36, 8

A. shengi Sze 1954

1977 Feng et al., pl.244, 5

A. subelegans (Potonié 1893) Franke 1912

1976 Barthel et al., pl.30, 7; pl.32, 10-12; pl.39, 3

A. virginiana Fontaine et White 1880

1978 Gillespie et al., pl.36, 2

A. yiyangensis Feng 1977

1977 Feng et al., pl.244, 3, 4

\section{Ammatophyllum Zalessky 1936}

A. sp.

1976 Bohlin, figs.301, 683; pl.17, 16 (all cf.)

\section{Amyelon Williamson 1874 ex Barnard 1962}

A. sp.

1980 Tian et Zhang, pl.25, 1-3; pl.26, $1 ;$ pl.27, 2-4

\section{Angaridium Zalessky 1933}

A. sp.

1976 Bohlin, fig.430; pl.21, 10-11 (all cf.)

1977 Zimina, pl.1, 9

\section{Angaropteridium Zalessky 1930}

A. sp.

1977 Zimina, pl.1, 10(?)

A. tyrganicum Zalessky 1936

1976 Zimina, fig. p.32; pl.5, 1-4

1977 Zimina, fig.13; pl.1, 8
India

(scheme)

(scheme from Halle, 1931)

China (Guizhou) UP

GDR (Suhl) LPAutu

(scheme) (UC-LP?)

USA (West Virginia) UC-LP?

China (Inner Mongolia) LP

(scheme)

China (Inner Mongolia) LP

China (Shanxi)

(scheme)

(scheme)

(UC-LP?)

GDR (Erfurt)

GDR (Karl-Marx-Stadt)

GDR (Suhl)

GDR (Karl-Marx-Stadt)

GDR (Karl-Marx-Stadt)

UPBara-Rani

(C-P)

(C-P)

P

$\mathrm{P}$

LPAutu

LPAutu

LPAutu

LPRotl

LPRot1

(from Sterzel, 1918)

(scheme)

(UC-LP?)

USA (West Virginia)

UC-LP?

China (Henan)

UP

GDR (Karl-Marx-Stadt)

LPRotl

USA (West Virginia)

UC-LP?

China (Henan)

LP

China (Chinghai)

UC-LP

China (Guizhou)

UP

China (Chinghai)

UC-LP

USSR (Primorskiy Kray)

LP

USSR (Primorskiy Kray) LP

USSR (Primorskiy Kray) UP

USSR (Primorskiy Kray) UP 
Ankyropteris (Stenzel 1889) Bertrand 1907

A. scandens (Stenzel 1889) Bertrand 1907

1976 Barthel et al., pl.2. 4, 5

LPRotl

Annularia Sternberg 1821

A. sp.

1976 Barthel et al., pl.27, 14

1976 Bohlin, fig.471 (cf.)

1976 Bohlin, fig.530(?); pl.1, 12(?)

1979 Asama, fig.13B

1979 Rösler, fig.4(1)

1979 Yuasa, fig.4.5

$1980 \mathrm{X}$. number " 2 " in fig.

A. asteris Bell 1924

1978 Gillespie et al.. pl.26, 5

A. carinata Gutbier 1837

1976 Barthel et al., pl.26, 5-8

1980 Barthel (a), fig.96(3.3A)

A. gracilescens Halle 1927

1977 Feng et al., pl.235, 8

A. hunanensis $\mathrm{Gu}$ et $\mathrm{Zhi} 1974$

1977 Feng et al., pl.235, 9

A. longissima Nejburg 1964

1977 Huang, pl.1, 12

A. mucronata Schenk 1883

1976 Huang, pl.211, 4

1977 Feng et al., pl.235, 10

A. pingloensis (Sze 1940) Gu et Zhi 1974

1977 Feng et al., pl.2338, 3, 4

1978 Chen et al., pl.150, 4

1978 Zhang, pl.152. 7

1980 Tian et Zhang, fig.17; pl.5, 1, 4, 5. 9

China (Chinghai)

LPRotl

China (Kansu)

UC-LP

$\mathrm{UC}$ - LP

(scheme from Abbott, 1958)

(C-P)

(scheme)

(scheme from Abbott, 1958)

(C-P)

(rec. from Bertrand. 1943)

USA (West Virginia)

UC $\cdot$ LP?

GDR (Karl-Marx-Stadt)

LPRotl

GDR (Gera)

LPRotl

China (Henan)

LP

China (Hunan)

UP

China (Heilongjiang)

LP

China (Inner Mongolia) LP

China (Hunan)

UP

China (Guangdong) UP

China (Sichuan) UP

China (Guizhou) UP

China (Guizhou) UP

China (Guizhou/Yunnan) UP

A. shirakii Kawasaki 1927

1978 Zhang, pl.152, 8

1980 Zhao et al., pl.2. 1012

A. sphenophylloides (Zenker 1833) Gutbier 1837

1976 Barthel et al., pl.24, 6; pl.26, 9

1977 Remy et Remy. fig. $227 d$

1978 Gillespie et al.. pl.26, 3, 6, 7

A. spicata (Gutbier 1849) Schimper 1869

1976 Barthel et al., pl.28. 199

1980 Barthel (a), fig.96(1/2)

A. stellata (Sternberg 1825) Wood 1860

1976 Barthel et al.. pl.24, 7; pl.25, 1, 2; pl.26, $1 ;$ pl.29, 11

1976 Huang, pl.211, 5, 6

1977 Krumbiegel et Walther, pl.48, 80

1977 Remy et Remy, fig.225b

1977 Remy et Remy, fig. $225 \mathrm{c}$

1978 Daber et Helms, figs. p.36, 39, 202, 206

1978 Gillespie et al.. pl.26, 1

1978 Remy et Remy (b), fig.1

1978 Strasburger, fig.737B

1980 Barthel (a), fig. $93(1 / 3)$

1980 Barthel et Haubold, pl.9, 3

1980 Bold et al., fig. 19-51

1980 El-Khayal et al., fig.2B

China (Guizhou)

UP

China (Guizhou/Yunnan)

UP

GDR (Karl-Marx-Stadt)

LPRotl

(scheme)

(C $\mathrm{P})$

USA (West Virginia)

$\mathrm{UC}-\mathrm{LP}$ ?

GDR (Karl-Marx-Stadt)

LPRotl

GDR (Gera)

L.PRotl

GDR (Karl-Marx-Stadt)

LPRotl

China (Inner Mongolia)

LP

GDR (Karl-Marx-Stadt)

LPRotl

(scheme)

(C. $\mathrm{P})$

(rec)

(C P)

(scheme)

USA (West Virginia)

(rec.)

(C P)

UC-LP?

(C) P)

(from Stur)

GDR (Gera)

$(\mathrm{C} \cdots \mathrm{P})$

L.PRotl

GDR (Suhl)

L.PAutu

$\mathrm{C} \cdots \mathrm{P}$

Saudi Arabia ('Unayzah)

UC-LP

A. tenuifolia Nejburg 1948

1979 Burago, pl.7, 4: pl.9, 1

USSR (Primorskiy Kray)

LP 
A. undulata Nejburg 1964

1976 Durante, pl.2, 1-3; pl.3, 2-4 (all cf.)

\section{Annulina Nejburg ex Rasskazova 1961}

+ A. planifolia fa. crassinervia Radchenko ex MS

1979 Burago, pl.6. 3, 4; pl.9, 2

USSR (Primorskiy Kray)

\section{Antarticoxylon Seward 1914}

A. sewardii Mussa 1980

1980 Mussa, pl.1, 1-4; pl.2, 5-8; pl.3, 9-12; pl.4, 13-17

Brazil (São Paulo)

$\mathbf{P}$

\section{Antevsia Harris 1937}

A. zeilleri (Nathorst 1908) Harris 1937

1979 Dilcher, fig.26

1979 Dilcher, fig.27 (pollen)

\section{Aphlebia Presl 1838}

A.erdmannii (Kunze in Germar 1849) Potonié 1893 1980 Barthel (c), pl.2, f.8, 9

\section{Araucaria Jussieu 1789}

A. sp.

1979 Lichter, fig.3(6)

(rec.)

\section{Araucarioxylon Kraus 1870}

A. loharense Agashe et Gowda 1978

1978 Agashe et Gowda, figs.1A-I, 2A-G

India (Maharashtra)

LG

\section{Araucarites Presl 1838}

A. permicus Mercklin 1855

1885 Quenstedt, pl.97, 15

A. saxonicus (Reichenbach 1836) Geinitz 1858

1885 Quenstedt, pl.97, 16

(from Ludwig)

\section{Arberia White 1908}

A. sp.

1978 Bernardes de Oliveira, pl.1, 2

A. brasiliensis Lundqvist 1919

1978 Bernardes de Oliveira, fig.10; pl.2, 2 (all aff.)

A. minasica White 1908

1976 Schopf, fig.11d

1977 Pant, fig.6G

1978 Bernardes de Oliveira, figs.1-9; pl.1, 1, 3, 4; pl.2,

$1,3,4 ; \mathrm{pl} .3,1-4 ; \mathrm{pl} .4,1$

$\begin{array}{ll}\text { Brazil (Santa Catarina) } & \text { L-MP } \\ \text { Brazil (Santa Catarina) } & \text { L-MP }\end{array}$

(from Rigby, 1972)

(rec. from Rigby, 1972)

Brazil (Santa Catarina) L-MP

\section{Arberiella Pant et Nautiyal 1960}

A. sp.

1976 Schopf, pl.3, 5; pl.4, 1-6

1977 Gould et Delevoryas, fig.8A-G

Remark: descr./ills. include anatomy and pollen grains.

1978 Van Dijk et al., fig.1

A. africana Pant et Nautiyal 1960

1977 Pant, fig.8F, G

1977 Pant, fig.11B

South Africa (Natal)

Australia (Queensland)

$\mathrm{P}$

UP

South Africa (Natal)

UP

(from Pant et Nautiyal, 1960)

India
LPRotl 
A. vulgaris Pant et Nautiyal 1960

1977 Chandra et Surange (d), fig.3, 5 (cuti, pollen); pl.1, 7 (pollen); pl.2, 8 (cuti), 9 (pollen), 10 (cuti), 13 (Faunipollenites)

India (West Bengal)

LGRani 1977 Pant, fig. $8 \mathrm{H}$

Remark: in situ pollen: Striatites.

(from Pant et Nautiyal, 1960)

\section{Arberiopsis Bernardes de Oliveira 1978}

A. sp.

1978 Bernardes de Oliveira, figs.11-13; pl.4. 2. 3, 5 (all ?)

A. boureaui Bernardes de Oliveira 1978

1978 Bernardes de Oliveira, figs. 14 16; pl.4, 4

Brazil (Santa Catarina)

L MP

Brazil (Santa Catarina)

I, -MP

\section{Archaeocycas Mamay 1973}

A. sp.

1979 Taylor et Millay, fig.3C

A. whitei Mamay 1973

1976 Mamay, figs.3. 7A, 8A

1976 Mamay, pl.1, 1 ; pl.2, 57

1980 Bold et al., fig. 2420

(from Mamay. 1976)

(rec.)

USA (Texas)

LP

\section{Arthropitys Goeppert 1864}

A. bistriata (Cotta 1832) Goeppert 1864

1976 Barthel et al., pl.28, 18.20

1977 Krumbiegel et Walther, pl.47. 78

1977 Marguerier, pl.2. 1. 2

A. communis (Binney 1868) Renault 1876

1978 Strasburger, fig. 735

1978 Strasburger, fig. 736

1980 Bold et al., fig. 1949

GDR (Karl-Marx-Stadt)

LPRotl

GDR (Karl-Marx-Stadt)

L.PRot]

France (Saône-et-Loire)

LP

(from Knoell)

(C-P)

(from Scott)

(C P)

(C $P$ )

\section{Artisia Presl 1838}

A. sp.

1978 Daber et Helms, figs. p.36, 241

1978 Mussa et al., pl.1, 14 (tipo)

A. approximata (Brongniart 1828) Corda 1838

1976 Barthel et al., pl.43, 5

(scheme)

Brazil (São Paulo)

GDR (Karl-Marx-Stadt)

India (West Bengal)

(scheme from Němejc)

China (Chinghai)

(scheme)

(C P)

LPRotl

A. sp.

1977 Feng et al. fig. 101

\section{Asterochlaena Corda 1845}

A. laxa Stenzel 1889

1976 Barthel et al., pl.2, 1; pl.11. 15 
A. sp.

1976 Bohlin, fig.667("')

China (Chinghai)

UC-LP

$1980 \mathrm{X}$, number " 3 " in fig.

(rec. from Bertrand, 1943)

A. dumasii Zeiller 1892

1980 Barthel (a), fig.95(2)

GDR (Gera)
GDR (Karl-Marx-Stadt)

LPRotl

A equisetiformis (Sternberg 1825) Brongniart 1828

1976 Barthel et al., pl.29, $6-8$

1978 Gillespie et al., pl.25, 3

LPRotl

USA (West Virginia)

UC-LP?

A. equisetiformis (Sternberg 1825) Brongniart 1828

fa. equisetiformis

1977 Remy et Remy, fig.229A

1980 Barthel (a), fig.94(4, 5)

A. longifolius (Sternberg 1825) Brongniart 1828

1978 Gillespie et al., pl.25, 5

GDR (Gera)

GDR (Gera)

LPAutu

LPRotl

USA (West Virginia)

UC-LP?

\section{Asterotheca Presl 1846}

A. sp.

1976 Bohlin, fig.501; pl.9, 15

1977 Remy et Remy, fig.56A

1979 Rösler, fig.4(6)

1979 Rösler, figs.7, 8

A. arborescens (Sternberg 1825) Stur 1877

1977 Remy et Remy, fig.119B

A. cottoni (Zeiller 1902) Hirmer 1927

1977 Feng et al., pl.242, 5

+ A. cyathea (Schlotheim 1804) Brongniart 1828

1977 Remy et Remy, fig.120A

1977 Remy et Remy, fig.120B

A. golondrinensis Herbst 1978

1978 Herbst, fig.1A-D; pl.1, 1-5

A. hemitelioides (Brongniart 1833) Stur 1877

1977 Remy et Remy, fig.121A

1977 Remy et Remy, fig.121B

A. primorskiensis Burago 1980

1980 Burago (a), fig.6; pl.9, 1-3 (all ?)

A. sternbergii (Goeppert 1836) Presl 1846

1976 Barthel et al., pl.18, 1-2

$\begin{array}{ll}\begin{array}{l}\text { China (Kansu) } \\ \text { (scheme) } \\ \text { (scheme) } \\ \text { Brazil (Paraná) }\end{array} & \begin{array}{l}\text { UC-LP } \\ \text { (C-P) }\end{array} \\ \text { GDR (Gera) } & \text { LP } \\ \text { China (Guangdong) } & \text { LPAutu } \\ \begin{array}{l}\text { GDR (Gera) } \\ \text { (scheme) }\end{array} & \text { UP } \\ \text { Argentina (Santa Cruz) } & \begin{array}{l}\text { LPAutu } \\ \text { (C-P) }\end{array} \\ \begin{array}{l}\text { GDR (Gera) } \\ \text { (scheme) }\end{array} & \text { LP } \\ \text { USSR (Primorskiy Kray) } & \begin{array}{l}\text { LPAutu } \\ \text { (C-P) }\end{array} \\ \text { GDR (Dresden) } & \text { UP }\end{array}$

\section{Australoxylon Marguerier 1973}

A. kanhargaoense Prasad et Chandra 1978

1978 Prasad et Chandra (b), figs.1-3

India (Maharashtra)

LGKamt

\section{Austroglossa Holmes 1974}

A. walkomii Holmes 1974

1976 Gould, fig.4A

1976 Schopf, fig.13a, b

1977 Pant, fig.10C

1980 Retallack, fig.21.5J ("'"; in situ)

(scheme from Holmes, 1974)

Australia (New South Wales) UP

(from Holmes, 1974)

Australia (New South Wales) L-UP

\section{Austroscleromedulloxylon Mussa 1980}

A. geraldinii Mussa 1980

Remark: no diagnosis given.

1980 Mussa et al., pl.1, 2-4

Brazil (São Paulo)

$\mathrm{P}$

Bachtia Nejburg 1960

$B . \mathrm{sp}$.

1976 Durante, pl.1, 1-4 
1978 Fefilova (b), fig. 17; pl.17, $1 \cdots 5$

1978 Fefilova (b), pl.17, 6

\section{Bageopitys Dohms 1976}

B. articulata Dohms 1976

1976 Dohms, figs.1-7; pls.1--5

\section{Bajdaievia Nejburg 1960}

B. longiuscula Fefilova 1978

1978 Fefilova (b), fig. $11 ;$ pl.10

B. speciosa Fefilova 1978

1978 Fefilova (b), fig.12; pl.11, 1,4

1978 Fefilova (b), pl.11, 2

1978 Fefilova (b), pl.11, 3

\section{Barakaroxylon Surange et Maithy 1962}

B. brasiliense Schrage 1973

1978 Guerra, fig.2A

B. jahriense (Surange et Sah 1956) Surange et Maithy 1962

1978 Guerra, fig.2D

B. lafoniense (Halle 1912) Schrage 1973

1978 Guerra, fig.2B

B. monocanalosum Kulkarni 1971

1978 Guerra, fig.2E

B. resiniferum Guerra 1978

1978 Guerra, figs.1A-D, 2C; pl.1, A-D; pl.2, A-F

\section{Bardocarpus Zalessky 1937}

+ B. iunguskaensis Verbitskaya

1976 Durante, pl.72, 12

\section{Belemnopteris Feistmantel 1877}

B. elongata Lacey, Van Dijk et Gordon-Gray 1975

1978 Rigby (b), figs.25, 26

1979 Van Dijk et al.. pl.47, 32-43

B. pellucida Pant et Choudhury 1977

1977 Pant et Choudhury, figs.4, 5; pl.5, 30-34; pl.6, 36-39

Remark: descr.iills. include cuticles.

B. sagittifolia Pant et Choudhury 1977

1977 Pant et Choudhury, figs.1-3; pl.1; pl.2, 7-12; pl.3; pl.4

Remark: descr./ills. include cuticles and anatomical details

\section{Bergeria Presl in Sternberg 1838}

B. sp.

1977 Feng et al., fig.101

1977 Feng et al., pl.233, 5

\section{Bicoemplectopteridium Asama 1959}

B. longifolium (Kodaira 1930) Asama 1959

1976 Asama, fig.1(6)

1976 Asama, pl.29, 3

1979 Asama, fig.3(6)

1980 Asama, fig.5

\section{Bicoemplectopteris Asama 1959}

B. hallei Asama 1959

1976 Asama, fig.1(3)
Mongolia

LP

USSR (Komi ASSR)

LPUfim

USSR (Komi ASSR)

UPKaza

Brazil (Rio Grande do Sul)

P

USSR (Komi ASSR)

UPKaza

USSR (Komi ASSR)

USSR (Komi ASSR)

USSR (Komi ASSR)

LPUfim

UPKaza

UPTata

$\begin{array}{ll}\text { Brazil (Rio Grande do Sul) } & \text { M - UP } \\ \text { India (Bihar) } & \text { LGBara } \\ \text { GB (Falkland Islands) } & \text { P } \\ \text { India (Bihar) } & \text { LGBara } \\ \text { Brazil (Rio Grande do Sul) } & \text { M U UP }\end{array}$

Australia (Queensland) U?P

South Africa (Natal) UP

India (West Bengal) UPRani

India (West Bengal) UPRani

(scheme) (C P)

China (Henan) UP

(scheme)

North? Korea (Kaisen.

Heian-Nando)

LP (scheme)

(scheme) 
1976 Asama, pl.28, 3, 3A

1976 Asama, pl.28, 3B

1976 Asama, pl.30, 6

1979 Asama, fig.2B

1979 Asama, figs.3(3), 4B

1979 Vozenin-Serra, pl.1, 4; pl.2, 5, 6

1980 Asama, fig.5

\section{Bifariusotheca Zhao 1980}

B. qinglongensis Zhao 1980

1980 Zhao, pl.12, 5-6

\section{Biscalitheca Mamay 1957}

B. dubius (Renault 1875) Galtier 1978

1978 Galtier, figs.A, B(5)

1978 Galtier, pl.1, 4; pl.2, 6, 8, 11-12 (spore)

\section{Bothrodendron Lindley et Hutton 1833}

$B$. henanense Xi 1977

1977 Feng et al., pl.235, 7

China (Henan)

LP

\section{Botrychiopsis Kurtz 1894}

B. sp.

1980 Andreis et al., pl.1, 6

B. ovata (McCoy 1847) Retallack 1980

1980 Retallack, fig.21.3G

B. plantiana (Carruthers 1860) Archangelsky et Arrondo 1971

1976 Gould, fig.3C, D

1980 Cazzulo-Klepzig et al. (b), pl.1, 4, 7, 13

1980 Retallack, fig. $21.3 \mathrm{~F}$

B. weissiana Kurtz 1894

1980 Retallack, fig.21.3E

\section{Botryopteris Renault 1875}

B. sp.

1977 Krumbiegel et Walther, pl.15, 25

B. burgkensis (Sterzel 1893) Barthel 1975

1976 Barthel (b), pl.1, 6; pl.2, 6, 7A, B; pl.3, 3, 3A, 6

1976 Barthel et al., pl.4, 1, 2; pl.5, 1-10; pl.6, 1-12,

13-19 (spores); pl.7, 1-14

\section{Bowmanites Binney 1871}

$B$. sp.

1976 Bohlin, fig.11; pl.1, 8-10

\section{Brachyphyllum Brongniart 1828}

B. sp.

1980 Retallack, fig.21.8I ("'))

Australia (New South Wales)

UP-LT

$\begin{array}{ll}\text { Brazil (Rio Grande do Sul) } & \text { LP } \\ \text { Australia (New South Wales) } & \text { UC-LP } \\ \text { (scheme) } & \text { (C-P) } \\ \text { Brazil (Rio Grande do Sul) } & \text { LPSakm } \\ \text { Australia (New South Wales) } & \text { UC-LP } \\ & \\ \text { Australia (New South Wales) } & \text { UC-LP }\end{array}$

(ill. on stamp)

GDR (Karl-Marx-Stadt)

LPRotl

GDR (Karl-Marx-Stadt)

LPRotl

China (Kansu)

UC-LP

\section{Brasilestiloxylon Mussa 1978}

B. piracicabense Mussa 1978

1978 Mussa (b), pl.1, 1-5

Brasilodendron Chaloner, Leistikow et Hill 1979

B. sp.

1979 Rösler, fig.4(4)

(scheme) 
B. africanum Lemoigne et Brown 1980

1980 Lemoigne et Brown (a), pl.3, 11 13, 1518

Namibia (Kaoko Veld)

U?P

B. pedroanum (Carruthers 1869) Chaloner, Leistikow et Hill 1979

1979 Chaloner et al., figs.1A C, $2 \mathrm{~A}-\mathrm{C}, 3 \mathrm{~A}, \mathrm{~B}, 4 \mathrm{~A}-\mathrm{C}^{\prime}$;

pl.1, 1-6; pl.2, 1 6; pl.3, 1-6; pl.4, 1 7, 8 (associated spore)

1980 Lemoigne et Brown (a), pl.3, 14 (cf.)

Brazil (Rio Grande do Sul)

L-MP

Namibia (Kaoko Veld)

$\mathrm{U}$ ?P

\section{Brongniartites Unger 1845}

B. sp.

1980 Durante, fig.3("')

China (Xizang)

UP

\section{Buriadia Seward et Sahni 1920}

B. sp.

1976 Balasundaram et al., pl.22, 15

1977 Maithy (c), pl.1, 2

1980 Guerra-Sommer et al., p1.1, 4

B. heterophylla Seward et Sahni 1920

1977 Correa da Silva et Arrondo, pl.2, 3

1980 Cazzulo-Klepzig et al. (a), pl.3, 1

1980 Cazzulo-Klepzig et al. (b), pl.2, 6

(scheme)

India (Bihar) UPKarh

Brazil (Rio Grande do Sul) IPSakm

Brazil (Rio Grande do Sul) LPSakm-Arti

Brazil (Rio Grande do Sul) LPSakm

Brazil (Rio Grande do Sul) LPSakm

\section{Calamariophyllum Hirmer 1927}

+ C. zeaeforme (Schlotheim 1820) Hirmer 1927

Remark: in text C. zeaeformis.

1980 Barthel (a), pl.97(3)

Remark: the specimen figured by Schlotheim (1820), pl.26, 2 .

GDR (Gera)

LPRotl

\section{Calamitea Cotta 1832}

C. bistriata Cotta 1832

1885 Quenstedt, pl.90, 10

GDR (Karl-Marx-Stadt)

LPRotl

\section{Calamites Brongniart 1828}

C. sp

1976 Barthel (b), pl.3, 2, 11

1976 Barthel et al., pl.27, 15

1976 Bohlin, figs.8, 527, 606, 607; pl.1, 6-7; pl.2, 12 ; pl.4,

$12-13$; pl.14, 8 (mostly cf., aff.)

1976 Bohlin, figs.286, 289-296, 575, 617, 618, 621, 679; pl.17, 1--5 (mostly cf.,?)

1976 Teixeira et Pais, figs.25A, B; 26A, B

1977 Feng et al., fig.104

1977 Feng et al., pl.236, 8

1977 Krumbiegel et Walther, fig.2.90.2

1977 Niklas, fig.4

1978 Gillespie et al., fig. 8

1978 Gillespie et al., fig.10C

1978 Ratkevich et La Fon, fig. p.49

1979 Asama, fig. 13A

1979 Major, fig.202

1979 Yuasa, fig.4.2

1980 Bold et al., fig. $19-48$ B;

1980 Bold et al., fig. $19 \cdot 50$

1980 Bold et al., fig. 19 52

1980 Tian et Zhang, fig. 3

$1980 \mathrm{X}$, numbers " 2,3 " in fig.

1980 Zhao et al., pl.4, 3

C. carinatus Sternberg 1824

1978 Gillespie et al., pl.23, 2, 5

$\begin{array}{ll}\text { GDR (Karl-Marx-Stadt) } & \text { LPRotl } \\ \text { GDR (Karl-Marx-Stadt) } & \text { LPRotl } \\ \text { China (Kansu) } & \text { UC-LP } \\ \text { China (Chinghai) } & \text { UC -LP } \\ \text { (rec.) } & \text { (C-P) } \\ \text { (scheme) } & \text { (C P) } \\ \text { China (Hunan) } & \text { UP } \\ \text { (scheme from Stur) } & \text { (C-P) } \\ \text { (scheme) } & \text { (C -P) } \\ \text { (rec.) } & \text { (UC-P) } \\ \text { (scheme) } & \text { (C-P) } \\ \text { USA (New Mexico) } & \text { LP } \\ \text { (scheme from Hirmer, 1927) } & \text { (C P) } \\ \text { (scheme) } & \text { (C-P) } \\ \text { (scheme from Hirmer, 1927) } & \text { (C - P) } \\ \text { (rec.) } & \text { (C-P) } \\ & \text { (C P) } \\ \text { (scheme from Eggert) } & \text { (C -P) } \\ \text { (rec.) } & \text { (C P) } \\ \text { (rec. from Bertrand, 1943) } & \text { (P) } \\ \text { China (Guizhou/Yunnan) } & \text { UP } \\ & \end{array}$

USA (West Virginia) UC LP? 
1980 Bold et al., fig.19-48A

C. cistii Brongniart 1828

1976 Huang, pl.218, 1

C. crassicaulis (Renault 1888) Kidston et Jongmans 1915

1976 Barthel et al., fig.9; pl.29, 1-3

C. gigas Brongniart 1828

1976 Barthel et al., pl.27, 8-11 (cf.), 12

1980 Barthel (a), fig.95(1, 3, 4, 6, 8)

1980 Barthel (a), fig.95(2)

1980 Barthel (a), fig.95(5)

1980 Barthel (a), fig.95(7)

C. infractus Gutbier 1835

1976 Barthel et al., pl.28, 17

C. multiramis Weiss 1884

1976 Barthel et al., pl.26, 2, 3

1980 Barthel (a), fig.93(1/9)

C. suckowii Brongniart 1828

1976 Barthel et al., pl.27, 13

1980 Barthel (a), fig.96(4)

\section{Calamitina Weiss 1876}

C. sp.

1978 Gillespie et al., figs.9, $10 \mathrm{I}$

1980 Barthel (a), fig.94(7)

\section{Calamitopsis Remy et Remy 1977 ex Remy et Remy 1978}

C. sp.

1977 Remy et Remy, fig.225C

C. multiramis (Weiss 1884) Remy et Remy 1977

1977 Remy et Remy, fig. 220

1978 Remy et Remy (b)

\section{Calamodendron Brongniart 1849}

C. sp.

1978 Daber et Helms, fig. p.206

C. striatum (Cotta 1832) Brongniart 1849

1976 Barthel et al., pl.26, 4; pl.29, 10

1976 Daber et Helms, fig. p.71

1978 Daber et Helms, fig. p.194

\section{Calamostachys Schimper 1869}

C. sp.

1976 Barthel et al., pl.26, 13

1977 Remy et Remy, fig.234C

1979 Yuasa, fig.4.4;

1979 Yuasa, fig. 4.6

C. binneyana (Carruthers 1867) Schimper 1869

1980 Bold et al., fig.19-53A, B

C. dumasii (Zeiller 1892) Jongmans 1911

1976 Barthel et al., pl.27, 1-4, 5-7 (spore), 8-11 (cf.), 16

1980 Barthel (a), fig.95(8)

C. tuberculata (Sternberg 1825) Weiss 1884

1976 Barthel (b), pl.1, 5; pl.2, 11-13

1976 Barthel et al., pl.25, 1-9, 10-13 (spore), 14; pl.26, 2

1977 Remy et Remy, fig.234D

1980 Barthel (a), figs.93(4-7, 8 (spore)); 97 (1, 1A, 2A-C)

Remark: fig. 97 is the specimen figured by Sternberg (1825), pl.45, 2 (rec. from Hirmer)

(C-P)

China (Inner Mongolia)

UC-LP

GDR (Karl-Marx-Stadt)

LPRotl

GDR (Karl-Marx-Stadt)

LPRotl

GDR (Erfurt)

LPRot]

GDR (Gera)

GDR (Karl-Marx-Stadt)

LPRotl

LPRotl

GDR (Suhl)

LPRotl

GDR (Karl-Marx-Stadt)

LPRotl

GDR (Karl-Marx-Stadt)

LPRotl

GDR (Gera)

LPRotl

GDR (Karl-Marx-Stadt)

LPRotl

GDR (Gera)

LPRotl

(scheme)

(C-P)

GDR (Gera)

LPRotl

(rec.)

(C-P)

GDR (Gera)

LPAutu

(rec.)

(C-P)

(scheme)

(C-P)

GDR (Karl-Marx-Stadt)

LPRotl

GDR (Karl-Marx-Stadt)

LP

GDR (Karl-Marx-Stadt)

LPRotl

LPRotl

(scheme)

(C-P)

(scheme from Williamson et Scott

1894)

(C-P)

(scheme from Schenk, 1883)

(scheme from Zimmermann) (C-P)

GDR (Karl-Marx-Stadt) LPRotl

GDR (Erfurt) LPRotl

GDR (Karl-Marx-Stadt) LPRotl

GDR (Karl-Marx-Stadt) LPRotl

GDR (Gera) LPAutu

GDR (Gera) LPRotl 


\section{Callipterianthus Roselt 1962}

C. arnhardtii Roselt 1962

1980 Haubold, pl.2, 2. 4

\section{Callipteridium Weiss 1870}

C. sp.

1978 Gillespie et al., pl.36, 4 (cf.)

USA (West Virginia)

UC LP

C. gigas (Gutbier 1849) Weiss 1870

1976 Barthel et al., pl.30, 24

GDR (Karl-Marx-Stadt)

LPRotl

1978 Gillespie et al., pl.38, 2

USA (West Virginia)

UC $\cdots P$

1978 Gillespie et al., pl.38, 3

+ C. pteridium (Schlotheim 1820) Zeiller 1888

(scheme)

(UC LP)

1978 Gillespie et al., pl.38, 1

USA (West Virginia)

UC- LP

C. tachingshanense (Sze 1934) Lee et al. 1974

1980 Huang, pl.223, 5 ; pl.224, 4-5

China (Liaoning)

$\mathrm{UC}-\mathrm{LP}$

\section{Callipteris Brongniart 1849}

C. sp.

1976 Durante, pl.44, 1; pl.47, 1, 4; pl.49, 7(?)

1976 Huang, pl.222, 6

1980 Durante, figs.2. 3

1980 Haubold, figs. $1,4,5 ;$ pl.1; pl.2, 3-5; pl.4, 1-5

1980 Haubold, fig. 2

1980 Haubold, fig. 3

$1980 \mathrm{X}$, number " 7 " in fig.

C. altaica Zalessky 1918

1976 Durante, pl.43, 12

C. angustata Zalessky 1939

1977 Huang, pl.29, 1; pl.43, 78

C. biforma Huang 1977

1977 Huang, fig. 12 ; pl.26, 3; pl.28, 1

C. conferta (Sternberg 1833) Brongniart 1849

1976 Barthel (b), pl.4, 3

1976 Barthel et al.. p].31, 5

1976 Denizot, pl.3, 14

1976 Mundlos et al., fig. p.89

1976 Mundlos et al. fig. p.90

1976 Rothe, fig.18

1976 Van Amerom et al., figs.2 (1-5), 3 ( 1 7)

1977 De la Peña et al., pl.1, 1, 2

1977 Krumbiegel et Walther, fig.2.95.5

1977 Remy et Remy, fig.160A

1977 Remy et Remy, fig.160B

1977 Sopeña et al., fig.4.1

1977 Tenchov (b), pl.1. 2; pl.2, 1, 2

1978 Daber et Helms, figs. p.11, p.152

1978 Gillespie et al., pl.39, 1-3

1979 Gillespie et Pfefferkorn, pl.3, 6 -8

1979 Richter, fig.171

1979 Schniepp, fig. 12

1980 Barthel et Haubold, fig.1

1980 Barthel et Haubold, figs.2 (A D) 12

1980 Barthel et Haubold, pl.1, 1; pl.4, 3; pl.13, 1 2

\begin{tabular}{|c|c|}
\hline Mongolia & $\mathrm{UP}$ \\
\hline China (Inner Mongolia) & UP \\
\hline China (Xizang) & UP \\
\hline GDR (Suhl) & LPAutu \\
\hline $\begin{array}{l}\text { CSSR (Ceské Zemé } \\
\text { "Bohemia" } \\
\text { (from Gothan, 1907) } \\
\text { (rec. from Mägdefrau, 1959) }\end{array}$ & LPAutu \\
\hline Mongolia & UP \\
\hline China (Heilongjiang) & UP \\
\hline China (Heilongjiang) & UP \\
\hline GDR (Karl-Marx-Stadt) & LPRotl \\
\hline GDR (Karl-Marx-Stadt) & LPRotl \\
\hline France $(\cdots)$ & LPAutu \\
\hline GFR (Saarland) & LPRotl \\
\hline GFR (Rheinland-Pfalz) & LPRotl \\
\hline GFR (Baden-Württemberg) & LPRotl \\
\hline Austria (Carinthia) & LP \\
\hline $\begin{array}{l}\text { Spain (Soria) } \\
\text { (scheme from Gothan et Remy) }\end{array}$ & LPAutu \\
\hline $\begin{array}{l}\text { GFR (Saarland) } \\
\text { CSSR (Ceské Zemé }\end{array}$ & LPAutu \\
\hline $\begin{array}{l}\text { CSSR (Ceské Zemé ... } \\
\text { "Bohemia" --) }\end{array}$ & LPAutu \\
\hline Spain (Guadalajara) & LPAutu \\
\hline $\begin{array}{l}\text { Bulgaria (Northwest) } \\
\text { (scheme) }\end{array}$ & LP \\
\hline USA (West Virginia) & LP? \\
\hline USA (West Virginia) & LP? \\
\hline France (Hérault) & LPAutu \\
\hline $\begin{array}{l}\text { GFR (Saarland) } \\
\text { (rec.) } \\
\text { (scheme) }\end{array}$ & LPRotl \\
\hline CSSR (Ceské Zemé & \\
\hline "Bohemia" ....) & LPAutu \\
\hline GFR (Saarland) & LPAutu \\
\hline GDR (Suhl) & LPAutu \\
\hline
\end{tabular}

1980 Barthel et Haubold, pl.1, 2; pl.2, 7; pl.3, 1; pl.14, 1; pl.15, 1-3 1980 Barthel et Haubold, pl.1, 3 5; pl.2, 4 6; pl.3, 2 7;

pl.4, 4; pl.9, 14 ; pl.12, 13

GDR (Suhl)

PAutu 
1980 Barthel et Haubold, pl.2, 1-3

1980 Haubold, pl.3, 1, 2

C. conferta (Sternberg 1833) Brongniart 1849 var. supaioides Haubold 1980

1980 Haubold, fig.1, 1; pl.2, fig.1

C. confluens Nejburg 1957

1976 Durante, pl.10, 6 (cuti); pl.41, 1-3; pl.42, 5, 6; pl.44, 4;

pl.45, 4; pl.46, 1; pl.48, 7 (ex gr.); pl.50, 5, 6; pl.53, 5 (ex gr.); pl.74, 4-8 (cuti)

1977 Huang, pl.23, 1 (cf.)

C. congermana Zalessky 1928

1980 Huang, pl.252, 5

C. flabellifera (Weiss 1879) Zeiller 1892

1976 Barthel (b), pl.4, 6

1976 Barthel et al, pl.31, 6

1977 Remy et Remy, fig. $162 \mathrm{~A}-\mathrm{C}$

1980 Remy et al., figs.1-8; pl.1, 1-3; pl.2, 1-3; pl.3, 1-3;

pl.4, 1-7; pl.5, 1-10

1980 Remy et al., pl.6, 1-3

C. heilongjiangensis Huang 1977

1977 Huang, pl.19, 2; pl.20, 1; pl.25, 2; pl.26, 2

C. hongshanensis Huang 1977

1977 Huang, fig. $13 ;$ pl.16, 4; pl.22, 3

1980 Huang, pl.256, 2

C. jerunakovensis Gorelova 1962

1976 Durante, pl.49, 1-2 (ex gr.)

1980 Huang, pl.256, 3-4 (cf.)

1980 Huang, pl.256, 5-6 (cf.)

C. lodevensis (Brongniart 1835) Zeiller 1898

1977 Tenchov (b), pl.2, 4

C. martinsii (Germar 1839) Zeiller 1906

1977 Schaumberg, fig.3

1978 Daber et Helms, fig. p.195

1980 Barthel et Haubold, pl.4, 1

1980 Barthel et Haubold, pl.4, 2

C. mongoliaensis Nejburg 1957

Remark: in text C. mongoliensis

1976 Durante, pl.43, 3; pl.44, 2, 3; pl.49, 4, 5

C. moorei (Elias 1936) Remy et Remy 1977

1977 Remy et Remy, fig.163A, B

C. naumannii (Gutbier 1849) Sterzel 1881

1976 Barthel (b), pl.4, 4, 5

1976 Barthel et al., pl.31, 7-8

1976 Barthel et al., pl.31, 9

1977 Remy et Remy, fig.161A

1977 Remy et Remy, fig.161B

1977 Tenchov (b), pl.2, 5; pl.3, 4

1980 Remy et al., fig.9

C. nicklesii Zeiller 1898

1977 Tenchov (b), pl.2, 3 (cf.)

1978 Remy et Remy (c), pl.8, 4--9 (cf.)

1980 Remy et al., fig. 10

C. obese Huang 1977

1977 Huang, pl.28, 2; pl.29, 2-3; pl.31, 3

1980 Huang, pl.256, 1

C. orientalis Zalessky 1928

1976 Durante, pl.45, 2; pl.46, 2-3; pl.47, 2, 5 (all cf.)

C. polymorpha (Sterzel 1879) Gothan 1910

1976 Barthel et al., pl.39, 13
France (Saône-et-Loire)

GDR (Gera)

LPAutu

LPAutu

GDR (Suhl)

LPAutu

Mongolia

UP

China (Heilongjiang) UP

China (Heilongjiang)

UP

GDR (Dresden)

GDR (Dresden)

GDR (Suhl)

USA (Kansas)

(from Weiss, 1879)

China (Heilongjiang) UP

China (Heilongjiang) UP

China (Heilongjiang) UP

Mongolia UP

China (Heilongjiang) UP

China (Jilin) UP

Bulgaria (Northwest) LP

GFR (Hessen) UPKupf

GDR (Halle) UPKupf

GDR (Halle) UPKupf

GFR (Hessen) UPZech

Mongolia UP

USA (Kansas) LPAutu

GDR (Dresden) LPRotl

GDR (Karl-Marx-Stadt) LPRotl

GDR (Leipzig) LPRotl

GDR (Halle) LPAutu

(scheme)

Bulgaria (Northwest) LP

(scheme from Gutbier, 1849)

Bulgaria (Northwest) LP

Italy (Brescia)

(scheme from Zeiller, 1898)

China (Heilongjiang) UP

China (Heilongjiang) UP

Mongolia UP

GDR (Leipzig) IPRotl 
C. pseudoshenshuensis Huang 1977

1977 Huang, pl.25, 1: pl.32, 1

China (Heilongjiang)

UP

1980 Huang, pl,254, $16 \mathrm{a}$

China (Heilongjiang)

UP

raymondii Zeiller 1906

1977 Sopeña et al., fig.4.2

Spain (Guadalajara)

LPAutu

C. sahnii Zalessky 1928

1976 Durante, pl.42, 1-4; pl.45, 3; pl.47, 3 (all ex gr.)

1980 Durante, fig. 3 (cf.)

Mongolia

UP

China (Xizang)

UP

C. scheibei Gothan 1907 var. scheibei

1977 Remy et .Remy, fig.164A-D

C. shenshuensis Huang ex Huang 1976

1976 Huang, pl.222, $7: \mathrm{pl} .223,1-5$

1977 Huang, fig.11; pl.21, 1-3; pl.22, 1-2; pl.23, 3; pl.26, 1

1980 Huang, pl.252, 1

1980 Huang, pl.252, 2- 4

C. tangwangheensis Huang 1977

1977 Huang, pl.23, 2; pl.30, 1-3; pl.31, 12

C. tatianaeana Zalessky 1934

1980 Huang, pl.258, 3

C. tongxingensis Huang 1980

1980 Huang, fig. $35 ;$ pl.255, $1-8$

C. weberi Sterzel 1896

1976 Barthel (a), pl.1, 3

C. zeilleri Zalessky 1918

1977 Huang, pl.26, 5; pl.34, 4; pl.42, 67

GDR (Erfurt)

LPAutu

China (Inner Mongolia) - UP

China (Heilongjiang) UP

China (Heilongjiang) UP

China (Liaoning) UP

China (Heilongjiang) UP

China (Heilongjiang) UP

China (Liaoning) UP

GDR (Karl-Marx-Stadt) LPAutu

China (Heilongjiang) UP

\section{Cardiocarpus Brongniart 1880}

C. sp.

1976 Balasundaram et al., pl.22, 14

1980 Tian et Zhang, pl.22, 5

C. cardiformis Verbitskaya 1980

1980 Verbitskaya (d), pl.17, 2-3(?)

C. elongatus Sterzel 1893

1976 Barthel et al., pl.39, 3

Remark: ? on Alethopteris subelegans

C. gutbieri Geinitz 1855

1977 Remy et Remy, fig. $40 \mathrm{~F}$

C. reniforme Geinitz 1858

Remark: in text C. reniformis

1976 Barthel et al, pl.43, 11

(scheme)

China (Guizhou)

UP

USSR (Krasnoyarskiy Kray

Tungusk Basin -..) LP

GDR (Karl-Marx-Stadt)

LPRot1

GDR (Gera)

LPAutu

GDR (Karl-Marx-Stadt)

LPRotl

\section{+Cardiolepis Nejburg 1965}

Remark: non Cardiolepis Wallroth 1822.

See Farr et al. (1979, p.290)

C. piniformis Nejburg 1965

1977 Meien, figs. $1 * 3 ;$ pl.7, 1-13; pl.8, 1-4

1977 Meien, fig.4

1978 Meien, figs. 13,4 (cuti), $5-10,10 \mathrm{k} \cdots 1$ (Vesicaspora);

pl.1, $1.8 ; \mathrm{pl} .2,10 \cdots 12$ (pollen in situ)

USSR (Komi ASSR - Pechora

Basin --)

UP

(rec.)

USSR (Komi ASSR - Pechora

Basin -)

UP

Cardioneura Zalessky 1934

C. pulchra (Nejburg 1948) Shvedov 1961

1979 Burago, pl.8, 2; pl.9, 5

USSR (Primorskiy Kray)

LP

C. tebenjkovii Nejburg 1961

1979 Burago, pl.7, 6; pl.8, 1, 3; pl.10, 1

USSR (Primorskiy Kray)

LP 


\section{+ Cardiopteris Schimper 1869}

Remark: non Cardiopteris Wallich ex Blume 1849 See Farr et al. (1979; p.290)

C. $\mathrm{sp.}$

1976 Bohlin, figs.396-398, 443-444, 453, 456, 458-459, 467, 486-487, 493-494, 654-657; pl.26, 5 (all cf.)

\section{Carpentiera Němejc et Augusta 1934}

C. frondosa (Göppert 1864-1865) Florin 1940

1976 Barthel (b), pl.4, 1

GDR (Dresden)

LPRotl

1976 Barthel et al., pl.42, 12, 13

\section{Carpolithes Brongniart 1822}

C. sp.

1976 Bohlin, figs.258, 260, 568, 703; pl.15, 8-9, 14; pl.16, 12;

pl.27, 21; pl.29, 1-2 (all cf.)

1976 Durante, pl.72, 11

1978 Chen et al., pl.153, 7

1978 Zhang, pl.164, 1

1980 Tian et Zhang, fig. $27 ;$ pl.5, 7, 8; pl.16, 3, 4; pl.22, 2-4

C. galopinii Stockmans et Mathieu 1939

1976 Bohlin, figs.245, 564; pl.15, 16 (all cf.)

C. glansiformis Mo 1980

1980 Zhao et al., pl.17, 3-4

C. minutus Zhao 1980

1980 Zhao et al., pl.22, 2, 2A

C. punclalus Zhao 1980

1980 Zhao et al., pl.1, 7-7b

C. speculatus Mo 1980

1980 Zhao et al., pl.15, $6 ;$ pl.17, $2 ;$ pl.18, 8, 9; pl.20, 6

$\begin{array}{ll}\text { China (Kansu) } & \text { UC-LP } \\ \text { Mongolia } & \text { UP } \\ \text { China (Sichuan) } & \text { UP } \\ \text { China (Guizhou) } & \text { UP } \\ \text { China (Guizhou) } & \text { UP } \\ \text { China (Kansu) } & \text { UC-LP } \\ \text { China (Guizhou/Yunnan) } & \text { UP } \\ \text { China (Guizhou/Yunnan) } & \text { UP } \\ \text { China (Guizhou/Yunnan) } & \text { UP } \\ \text { China (Guizhou/Yunnan) } & \text { UP } \\ & \\ & \\ \text { (scheme) } & \\ \text { China (Hunan) } & \text { UP } \\ \text { China (Guangdong) } & \text { UP } \\ \text { (scheme) } & \\ \begin{array}{l}\text { China (Shanxi) } \\ \text { Spain (Soria) } \\ \text { China (Henan) }\end{array} & \text { LP } \\ \text { (at) } & \end{array}$

\section{Cathaysiopteris Koidzumi 1934}

C. sp.

1977 Feng et al., fig.107, 1

1977 Feng et al., pl.246, 5

C. huapingensis Feng 1977

1977 Feng et al., pl.246, 2

C. whitei (Halle 1927) Koidzumi 1934

1976 Asama, fig.1(9)

1976 Asama, pl.29, 6, 6A

1977 De la Peña et al., pl.1, 3-5

1977 Feng et al., pl.253, 6

1979 Asama, fig.3(9)

1980 Asama, fig. 5

(scheme)

(scheme)

\section{Cathaysioxylon Vozenin-Serra 1978}

C. khmerinum (Serra 1966) Vozenin-Serra 1978

1978 Vozenin-Serra, pl.1. 1-6; pl.2, 1-7

Cambodia (-)

UPKaza

\section{Caulophyllites Pant et Singh 1979}

C. indica Pant et Singh 1979

1979 Pant et Singh, fig.1

1979 Pant et Singh, figs.2, 3; pl.1, 1-5; pl.2, 6-12; pl.3, 13-19

(rec.)

India (Bihar)

LGKarh

\section{Caulopteris Lindley et Hutton 1832}

C. sp.

$1980 \mathrm{X}$, number " 5 " in fig.

(rec. from Corsin, 1948) 
C. varians Zeiller 1888

1978 Daber et Helms, figs. p.36, p.191

Chansitheca Regè 1921

C. sp.

1980 Tian et Zhang, pl.7, 5 5a(?)

China (Guizhou)

UP

C. kidstonii Halle 1927

1980 Zhao et al., pl.7. 1, 1a

Chapchalia Bohlin 1976

C. sp.

1976 Bohlin, figs.665 666, 668-669; pl.24, 9; pl.26, 9 (all cf.,?)

China (Chinghai)

UC-LP

C. tsaidamensis Bohlin 1976

1976 Bohlin, figs.446 449, 466, 470, 472, 475 484, 663, 664, 672,

$729 ;$ pl.24, $38 ;$ pl.25, 1 $6 ;$ pl.26, 13

China (Chinghai)

$\mathrm{UC}-\mathrm{LP}$

\title{
Cheirophyllum Pant et Singh 1978
}

C. lacerata (Feistmantel 1886) Pant et Singh 1978

1978 Pant et Singh, figs.1, 2 (cuti); pl.1, 1 7; pl.2, 8, 9, 10 16 (cuti) India (Bihar)

LGKarh

\section{Chiropteris Kurr 1858}

C. sp.

1976 Bohlin, fig.196; pl.32, 13 (all cf.)

1980 Andreis et al, pl.2, 1.

1980 Cazzulo-Klepzig et al, (a), pl.3, 3

1980 Cazzulo-Klepzig et al. (b), pl.1, 10

UC-LP

China (Kansu)

LP

Brazil (Rio Grande do Sul)

LPSakm

Brazil (Rio Grande do Sul)

LPSakm

C. reniformis Kawasaki 1925

1977 Dolianiti, figs. 14

1977 Feng et al., pl.245, 1, 2

1980 Cazzulo-Klepzig et al. (a), pl.2, 4, 6

Brazil (Santa Catarina)

$P$

China (Henan)

UP

Brazil (Rio Grande do Sul)

LPSakm

\section{Chulakia Sal'menova 1979}

C. alveolata Sal'menova 1979

1979 Salmenova, pl.13. $5 \cdot 7$

C. pilifera Sal'menova 1979

1979 Sal'menova, pl.13. 8-10

\author{
USSR (Kazakhstan \\ Dzhungariya ...) \\ USSR (Kazakhstan \\ Dzhungariya ..)
}

UP

UP

\section{+ Cistella Plumstead 1958}

Remark: nom. rej. vs. Cistella Quélet 1886 (nom. cons.). See Farr et al. (1979, p.373).

C. sp.

1977 Bose et al., pl.2, 16 17(?)

C. ampla White 1963

1978 White, fig.65

1978 White, fig.66

C. stricta Plumstead 1958

1976 Schopf, fig. 1(".")

India (Madhya Pradesh)

UP LT

Australia (New South Wales)

$\mathrm{P}$

Australia (Queensland)

$\mathrm{P}$

(scheme)

\section{Cladophlebis Brongniart 1849}

C. sp.

1976 Bohlin, fig.A on p.44( $\left.{ }^{+*}\right)$

1976 Bohlin, figs.531, 545, 695; pl.11, 2, 10, 13; pl.12, 3, 12

(all cf., aff.,?,"')

1976 Durante, pl.20, 3

C. connexa Zhang 1978

1978 Zhang, pl.157, 57

(scheme)

China (Kansu) UC - LP

Mongolia UP

China (Guizhou) UP 
C. fuyuanensis Zhang 1980

1980 Zhao et al., pl.13, 3-4a

China (Guizhou/Yunnan)

UP

C. gorskii Vladimirovich 1980

1980 Vladimirovich, pl.11, 2

USSR (Komi ASSR - Pechora

Basin -)

UP

C. heishanensis Bohlin 1976

1976 Bohlin, figs.181, 183, 185, 187, 189, 190, 193, 195, 515; pl.4, 2;

pl.8, 11; pl.11, 12; pl.12, 5; pl.14, 2; pl.31, 11 (partly ef.)

China (Kansu) UC-LP

C. henanensis Zhang et Mo 1979

1979 Zhang et Mo, pl.2, 2-5

China (Henan) UP

C. licharevii Vladimirovich 1980

1980 Vladimirovich, pl.10, 4; pl.11, 1

USSR (Kemerovskaya oblast --

Kuzbass -)

UP

C. manchurica (Kawasaki 1934) Gu et Zhi 1974

1976 Huang, pl.217, 1, 2

1980 Huang, pl.235, 6, 7

C. melnikovii Burago 1980

1980 Burago (b), fig. 9 ; pl.13, 1, 2

China (Inner Mongolia)

LP

China (Liaoning)

L?-UP

C. mongolica Durante 1976

USSR (Primorskiy Kray)

UP

Remark: see also C. mongolica Durante 1971 in IFPM, Permian 1971-1975, p.12.

1976 Durante, fig.27; pl.19, 7, 8; pl.21, 3, 4

C. nebbensis (Brongniart 1828) Nathorst 1875

1976 Le Roux, pl.1, 6-7 (cf.)

C. nystroemii Halle 1927

1977 Feng et al., pl.243, 9 (cf.)

C. ozakii Yabe et Oishi 1938

1978 Zhang, pl.157, 4

1979 Vozenin-Serra, pl.3, 15, 16

1980 Zhao et al., pl.12, 11, 11A

C. parapermica Chan MS ex Zhang 1978

1978 Zhang, pl.156, 6

1980 Zhao et al., pl.13, 1-2A

C. permiensis Lee et Wang 1956

Remark: in text C. permica.

1977 Feng et al., pl.243, 11

1978 Zhang, pl.156, 2

1980 Huang, pl.228, 4-5; pl.229, 3

1980 Zhao et al., pl.13, 5

C. xiaohinganensis Huang 1980

1980 Huang, pl.261, 3, 3A

C. yongwolensis (Kawasaki 1927-1931) Stockmans et Mathieu 1939

1976 Huang, pl.219, 2-3(?)

1980 Huang, pl.229, 4(?)

C. yunnanica 1980

1980 Zhao et al., pl.13, 6, 6A

Mongolia

UP

South Africa (Transvaal) LP

China (Henan) LP

China (Guizhou) UP

Laos (-) P

China (Guizhou/Yunnan) UP

China (Guizhou) UP

China (Guizhou/Yunnan) UP

China (Henan) UP

China (Guizhou) UP

China (Liaoning) UP

China (Guizhou/Yunnan) UP

China (Heilongjiang) UP

China (Inner Mongolia) LP

China (Liaoning) UP

China (Guizhou/Yunnan) UP

\section{Cladostrobus Zalessky 1918}

C. sp.

1980 Goman'kov et Meien, fig.1(5) (scheme)

C. lutuginii Zalessky 1918

1976 Durante, pl.71, 13

Mongolia

UP

Comia Zalessky 1934

C. sp.

1976 Durante, pl.43, 4; pl.45, 1; pl.49, 6; pl.50, 1; pl.51, 4-6 (partly ?) Mongolia 
C. enisejevensis Shvedov 1960

1976 Durante, pl.48, 1 (aff.), 2 4, 5-6 (aff.); pl.50, 1-4

Mongolia

UP

C. major Shvedov 1961

1977 Huang, fig.8; pl.9. 4

C. microphylla Huang 1977

1977 Huang, pl.15, 1 2; pl.16, 1-3; pl.28, 3

C. multinervis Huang 1977

1977 Huang, fig.9; pl.13. 1

C. obese Huang 1977

1977 Huang, fig. 10; pl.19, 1

C. osinowskiensis (Khakhlov 1931) Radehenko 1955

1980 Huang, pl.257, 8 (cf.)

C. shenshuensis Huang, 1977

1977 Huang, pl.17, 3; pl.18, 12

C. tenueaxis Huang 1977

1977 Huang, pl.11, 8; pl.12, 1 3

1980 Huang, pl.257, I 6

China (Heilongjiang) UP

China (Heilongjiang) UP

China (Heilongjiang) UP

China (Heilongjiang) UP

China (Liaoning) UP

('hina (Heilongjiang) UP

China (Heilongjiang) UP

China (Liaoning) UP

China (Heilongjiang) UP

China (Heilongjiang) UP

China (Liaoning) UP

Huang, pl.260. 1

1980 Huang, pl.260. 4

\section{Compsopteris Zalessky 1934}

C.sp.

1976 Durante, pl.41, 4: pl.49, 3

1980 Durante, fig. 3

1980 Tian et Zhang, pl.13, 3(?)

C. adzvensis Zalessky 1934

1977 Huang, pl.33, 12 (cf.)

C. contracta Gu et Zhi 1974

1977 Feng et al., pl.245, 6, 7

1977 Huang. pl.19, 3 (cf.); pl.33, 3 (cf.)

Remark: in text $C$. contracus

1978 Chen et al., pl.152, 23

1978 Zhang, pl.159, 1 (cf.), 23

1980 Tian et Zhang, pl.14, 4, 4A, 5

C. guangdongensis Feng 1977

1977 Feng et al., pl.241, 7, 8

C. imparis Gu et Zhi 1974

1977 Feng et al., pl.241, 6

1978 Zhang, pl.158, 5

C.punctinervis Mo 1980

1980 Zhao et al., pl.18. 15

C. tchirkovae Zalessky 1934

1977 Huang, pl.34, 1 3; pl.35, 1-2, 3(?)

C. wongii (Halle 1927) Zalessky 1934

1977 Feng et al., pl.245, 3. 4

1978 Zhang, p1.158, 2

$\begin{array}{ll}\text { Mongolia } & \text { UP } \\ \text { China (Xizang) } & \text { UP } \\ \text { China (Guizhou) } & \text { UP } \\ & \text { UP } \\ \text { China (Heilongjiang) } & \\ \text { China (Guangdong) } & \text { UP } \\ \text { China (Heilongjiang) } & \text { UP } \\ \text { China (Sichuan) } & \text { UP } \\ \text { China (Guizhou) } & \text { UP } \\ \text { China (Guizhou) } & \text { UP } \\ & \\ \text { China (Guangdong) } & \text { UP } \\ & \\ \text { China (Henan) } & \text { UP } \\ \text { China (Guizhou) } & \text { UP } \\ \text { China (Guizhou/Yunnan) } & \text { UP } \\ \text { China (Heilongjiang) } & \text { UP } \\ \text { China (Henan) } & \text { UP } \\ \text { China (Guizhou) } & \text { UP }\end{array}$

\section{+Conchophyllum Schenk 1883}

Remark: non Conchophyllum Blume 1826.

See Farr et al. (1979, p.410)

C. sp.

1976 Bohlin, figs.31 33, 612 (all cf.)

1976 Bohlin, figs.423, 635; pl.19, 8 (all ?)

C. parvifolium Bohlin 1971

1976 Bohlin, fig.30 (cf.)

China (Chinghai)

UC LP

$\mathrm{UC}$ - LP

China (Kansu)

UC LP

C. tsaidamense Bohlin 1976

1976 Bohlin, figs.337, 340-365, 367--370, 583-592, 596, 630-634;

pl.19, 7(?), 11; pl.20, 1-6; pl.22, 1-5; pl.28, 3-4, 8(?); pl.29,

$68 ; \mathrm{pl} .32,5 \div 6$

China (Chinghai)

$\mathrm{UC} \cdots \mathrm{LP}$ 
Cordaianthus Grand'Eury 1877

C. sp.

1976 Bohlin, figs.265-267, 514 (all ?)

$\begin{array}{ll}\begin{array}{l}\text { China (Kansu) } \\ \text { (scheme) } \\ \text { (scheme) }\end{array} & \begin{array}{l}\text { UC-LP } \\ \text { (UC-T) } \\ \text { (C-P) }\end{array} \\ \text { GDR (Karl-Marx-Stadt) } & \text { LPRotl } \\ & \\ \text { GDR (Karl-Marx-Stadt) } & \text { LPRotl }\end{array}$

1978 Gillespie et al., pl.53, 8

1980 Keng, figs.2, 20-24

C. baccifer Grand'Eury 1877

1976 Barthel et al., pl.43, 9, 12

Remark: pl.43, 9 on axis of Cordaites sp.

C. gemmifer Grand'Eury 1877

1976 Barthel et al., pl.43, 1

Remark: on axis with leaves of Cordaites principalis-borassifolius type

C. penjoni Renault 1879

1980 Keng, fig.5

C. pseudofluitans Kidston 1922

1976 Harris, fig. $1 \mathrm{~A}$

1978 Grauvogel-Stamm, fig.44A

1980 Keng, figs.3, 4

C. zeilleri Renault 1879

1978 Grauvogel-Stamm, fig.44B

\author{
LPRotl
}

$\begin{array}{ll}\text { (scheme) } & \text { (C-P) } \\ \begin{array}{l}\text { (scheme) } \\ \text { (scheme from Schweitzer, 1963) } \\ \text { (scheme) }\end{array} & \begin{array}{l}\text { (C-P) } \\ \text { (C-LP) }\end{array} \\ \text { (c-P) } \\ \text { (scheme from Schweitzer, 1963) } & \text { (C-LP) }\end{array}$

Cordaicarpus Zeiller 1886-1888

C. sp.

1976 Balasundaram et al., pl.22, 14

1976 Bohlin, pl.27, 21

1977 Appert, pl.18, 8, 9; pl.32, 6, 7; pl.35, 6

(scheme from Schweitzer, 1963)

(scheme)

China (Kansu) UC-LP

$\begin{array}{ll}\text { Madagascar (Sakoa Basin) } & \text { LGKarh-Bara? } \\ \text { Brazil (Santa Catarina) } & \text { LPArti }\end{array}$

1977 Bernardes de Oliveira et Da Silva Pontes, figs.13-14; pl.4, 5

1980 Andreis et al., pl.2, 7-8(?)

Brazil (Santa Catarina)

Brazil (Rio Grande do Sul)

Brazil (Rio Grande do Sul)

1980 Guerra-Sommer et al., pl.1, 7

C. irapuensis Bernardes de Oliveira 1977

1977 Bernardes de Oliveira et Da Silva Pontes, pl.4, 6-8

C. madagascariensis Appert 1977

1977 Appert, figs.5-10; pl.36, 3-10; pl.37, 1-6; pl.38/40

Remark: attached to Dolianitia madagascariensis

C. rocha-camposii Bernardes de Oliveira 1977

1977 Bernardes de Oliveira et Da Silva Pontes,

figs.11-12; pl.4, 9-11

Brazil (Rio Grande do Sul)

$$
\text { LP }
$$

LPSakm

LPSakm

Brazil (Santa Catarina) LPArti

Madagascar (Sakoa Basin)

LGKarh-Bara?

Brazil (Santa Catarina)

LPArti

USSR (Krasnoyarskiy Kray Tungusk Basin -)

LP

C. zeilleri Maithy 1965

1977 Bernardes de Oliveira et Da Silva Pontes, figs. $7-10 ;$ pl.4, 1-4

Brazil (Santa Catarina)

LPArti

\title{
Cordaicladus Grand'Eury 1877
}

C. sp.

1976 Durante, pl.57, 9

1978 Gillespie et al., pl.53, 9

Mongolia

UP

(scheme)

(UC-T)

\section{Cordaites Unger 1850}

C. sp.

1976 Barthel (b), pl.2, 15; pl.3, 1, 2

1976 Barthel et al., figs.16, 17; pl.43, 1-4, 7; pl.44, 1,

$1 \mathrm{~A}-9 ; \mathrm{pl} .45,1 \mathrm{D}-11$

Remark: descr./ills. include cuticles

1976 Bohlin, figs.244, 246-250, 255, 257, 261-263, 268, 271,

550(?), 559-562("'”), 563(?), 611(?), 728('"'); pl.14, 7;

pl.15, 1-6; pl.29, 14; pl.32, 16

Remark: all plates with "'" and?

GDR (Karl-Marx-Stadt)

GDR (Karl-Marx-Stadt)

China (Kansu)

UC-LP
LPRotl

LPRotl 
1976 Bohlin, figs.389, 653 (all ?)

1976 Bohlin, fig.693

1976 Durante, pl.68, 4 6; pl.69, 4-7

1976 Escher et Watt, fig.425A

1976 Mundlos et al., fig. p.90

1976 Paliwal et al., fig. I

1976 Teixeira et Pais, fig.36

1977 Krumbiegel et Walther, pl.6, 10

1977 Zimina, fig.16A L, M-N(?); pl.18, $1-3(?), 4,5$;

1977 Zimina, pl.4, 3-4

1978 Belot, fig. p.74

1978 Gillespie et al., figs.14, 15

1978 Gillespie et al., pl.54, 2

1978 Strasburger, fig. $788 \mathrm{~A}$

1979 Asama, fig.12A-D

1979 Delevoryas, fig. 9

1979 Major, fig. 211

1979 Yuasa, fig.57A

1979 Yuasa, fig.57B

1979 Yuasa, fig.57C, D

1980 Andreis et al., pl.1, 5, 8

1980 Cazzulo-Klepzig et al. (a), pl.1, 3; pl.2, 7; pl.3, 12

1980 Goman'kov et Mejen, fig.1(2)

1980 Guerra-Sommer et al., pl.1, 2, 3

1980 Keng, figs. 1, 20. 24

$1980 \mathrm{X}$, number " 8 " in fig.

C. adleri (Radchenko 1934) Meien 1966

1976 Durante, pl.69, 1-3 (cf.)

C. buragoi Zimina 1977

1977 Zimina, pl.6, 6, 7

C. foliolatus Grand'Eury 1877

1976 Barthel et al., pl.43, 8

C. gracilentus (Gorelova 1960) Meien 1966

1976 Durante, pl.67, 1, 2; pl.68, 2-3 (aff.)

1978 Durante et Biterman, pl.23, 13 (ex gr.)

C. hislopii (Bunbury 1861) Seward 1907

1980 Cazzulo-Klepzig et al. (b), pl.2, 5(?)

C. kuznetskianus (Gorelova 1962) Meien 1966

1976 Durante, pl.68, 1

C. laevis Grand'Eury 1877

1978 Strasburger, fig. $788 \mathrm{~B}$

C. latifolius (Nejburg 1948) Meien 1966

1976 Durante, pl.66. 4.6

1977 Zimina, pl.10, 7 (aff.)

C. $\operatorname{minax}$ (Gorelova 1960) Meien 1966

1978 Durante et Biterman, pl.23, 4-6 (ex gr.)

C. odontophyllus Glukhova 1978

1978 Glukhova, pl.11, 4

C. primorskiensis Zimina 1976

1976 Zimina (b), pl.7, 2 7: pl.8, 1 5

1977 Zimina, pl.10, 4 6; pl.11, 13

C. principalis (Germar 1848) Geinitz 1855

1977 Feng et al., pl.252, 1. 2

1978 Chen et al., pl.155, 3

1980 El-Khayal et al., fig.2A (cf.)

1980 Huang, pl.222, 1

$\begin{array}{ll}\begin{array}{l}\text { China (Chinghai) } \\ \text { (from Florin, 1949) }\end{array} & \text { UC-LP } \\ \text { Mongolia } & \text { UP } \\ \text { Greenland (East) } & \text { C-P } \\ \text { GFR (Rheinland-Pfalz) } & \text { LPRotl } \\ \text { (scheme) } & \\ \text { (rec. from Jongmans, 1949) } & \text { (C-T) } \\ \text { GDR (Karl-Marx-Stadt) } & \text { LPRotl } \\ \text { USSR (Primorskiy Kray) } & \text { LP } \\ \text { USSR (Primorskiy Kray) } & \text { UP } \\ \text { (scheme) } & \text { (D-T) } \\ \text { (rec.) } & \text { (UC-T) } \\ \text { (scheme) } & \text { (UC-T) } \\ \text { (rec. from Grand'Eury) } & \text { (C-P) } \\ \text { (scheme) } & \text { (D-P) } \\ \text { (from Florin, 1951) } & \text { (C P) } \\ \text { (scheme) } & \text { (D-T) } \\ \text { (scheme from Grand'Eury. 1877) } & \text { (C-P) } \\ \text { (scheme from Fly, 1956) } & \text { (C-P) } \\ \text { (scheme from Florin, 1944) } & \text { (C-P) } \\ \text { Brazil (Rio Grande do Sul) } & \text { LP } \\ \text { Brazil (Rio Grande do Sul) } & \text { LPSakm } \\ \text { (scheme) } & \text { LPSakm } \\ \text { Brazil (Rio Grande do Sul) } & \text { (C-P) } \\ \text { (scheme) } & \\ \text { (rec from Bertrand, 1943) } & \end{array}$

(rec. from Bertrand, 1943)

Mongolia

UP

USSR (Primorskiy Kray) UP

GDR (Karl-Marx-Stadt) LPRotl

\begin{tabular}{|c|c|}
\hline Mongolia & $\mathrm{UP}$ \\
\hline $\begin{array}{l}\text { USSR (Yakutskaya ASSR - } \\
\text { Verkhoyansk -) }\end{array}$ & UP \\
\hline Brazil (Rio Grande do Sul) & LPSakm \\
\hline Mongolia & UP \\
\hline (from Grand'Eury) & $(\mathrm{C} \cdots \mathrm{P})$ \\
\hline Mongolia & LP \\
\hline USSR (Primorskiy Kray) & LP \\
\hline $\begin{array}{l}\text { USSR (Yakutskaya ASSR } \\
\text { Verkhoyansk - }\end{array}$ & UP \\
\hline $\begin{array}{l}\text { USSR (Krasnoyarskiy Kray } \\
\text { Tungusk Basin }\end{array}$ & L.P \\
\hline $\begin{array}{l}\text { USSR (Primorskiy Kray) } \\
\text { USSR (Primorskiy Kray) }\end{array}$ & $\begin{array}{l}\text { L - UP } \\
\mathrm{LP}\end{array}$ \\
\hline China (Hunan) & UP \\
\hline China (Sichuan) & UP \\
\hline Saudi Arabia ('Unayzah) & UC $\cdots$ LP \\
\hline China (Liaoning) & UP \\
\hline
\end{tabular}


C. spathulata (Dana 1849) Rigby, Maheshwari et Schopf 1980

Remark: in text C. spatulata

1980 Rigby et al., figs. $48-50$

C. tologoiensis Durante 1976

1976 Durante, pl.67, 3-5; pl.74, 2 (cuti)

C. torulosus Glukhova 1980

1980 Glukhova et Men'shikova, pl.13, 5-7

C. truncatus Meien 1966

1976 Durante, pl.70, 1-3

C. yujiaensis Huang 1980

1980 Huang, fig.45; pl.222, 2

Cornucarpus Arber 1914

C. sp.

1980 Zhao et al., pl.1, 6, 6A

C. cerquilhensis Millan 1977

1977 Millan, pl.1, 1-7; pl.2, 1; pl.3, 1

C. patagonicus (Feruglio) Correa da Silva et Arrondo 1977

1977 Correa da Silva et Arrondo, pl.1, 6

1980 Cazzulo-Klepzig et al. (b), pl.1, 6

\section{Cornuspermum Banerjee 1969}

C. pennatus Banerjee 1969

1979 Banerjee, pl.50, 11-14

1979 Banerjee, pl.54, 8

Remark: in situ from Senotheca murulidihensis

\section{Corynepteris Baily 1860}

C. sp.

1976 Bohlin, fig.38; pl.3, 8 (all ?)

1976 Bohlin, figs.372-373, 375, 605; pl.24, 2; pl.26, 4;

pl.28, 5-7 (mostly ?)

C. kansuensis Bohlin 1976

1976 Bohlin, fig.36; pl.3, 1; pl.32, 2 (cf.); 17(?)

C. parilobata Bohlin 1976

1976 Bohlin, figs.379-383, 717; pl.29, 13 (all ?e"))

C. plicata Bohlin 1976

1976 Bohlin, figs.371, 603 (cf.); pl.21, 1, 6, 7

C. sternbergii (Ettingshausen 1854) Arber 1907

1976 Bohlin, fig.37 (aff.); pl.2, 9 (cf.)

1976 Bohlin, figs.473-474, 488-492, 658 (all cf.)

C. tridentata Bohlin 1971

1976 Bohlin, figs.374, 376-378; pl.22, 6-8 (all ? aff.)

\section{Crassinervia Nejburg 1934}

C. sp.

1976 Durante, pl.70, 5-8, 12

1977 Zimina, pl.19, 11

1980 Goman'kov et Meien, fig.1(4)

1980 Huang, pl.231, 5

C. grammii Zimina 1977

1977 Zimina, fig.21; pl.17, 8

1978 Zimina, pl.1, 6

C. mishanensis Huang 1980

1980 Huang, pl.248, 18-20

C. neuburgiana Zimina 1973

1977 Zimina, fig.22; pl.10, 8; pl.17, 9-13 (all ?)

1978 Zimina, pl.1, 8(?)

Remark: in text Zamiopteris (Crassinervia?) neuburgiana

\begin{tabular}{|c|c|}
\hline Australia (New South Wales) & $P$ \\
\hline Mongolia & UP \\
\hline $\begin{array}{l}\text { USSR (Kemerovskaya oblast - } \\
\text { Kuzbass -) }\end{array}$ & UP \\
\hline Mongolia & UP \\
\hline China (Liaoning) & LP \\
\hline China (Guizhou/Yunnan) & UP \\
\hline Brazil (São Paulo) & LP \\
\hline $\begin{array}{l}\text { Brazil (Rio Grande do Sul) } \\
\text { Brazil (Rio Grande do Sul) }\end{array}$ & $\begin{array}{l}\text { LPSakm-Arti } \\
\text { LPSakm }\end{array}$ \\
\hline
\end{tabular}

India (West Bengal)

UPRani

(rec.)

$\begin{array}{lr}\text { China (Kansu) } & \text { UC-LP } \\ \text { China (Chinghai) } & \text { UC-LP } \\ \text { China (Kansu) } & \text { UC-LP } \\ \text { China (Chinghai) } & \text { UC-LP } \\ \text { China (Chinghai) } & \text { UC-LP } \\ \text { China (Kansu) } & \text { UC-LP } \\ \text { China (Chinghai) } & \text { UC-LP } \\ \text { China (Chinghai) } & \text { UC-LP }\end{array}$

$\begin{array}{ll}\begin{array}{l}\text { Mongolia } \\ \text { USSR (Primorskiy Kray) } \\ \text { (scheme) }\end{array} & \text { UP } \\ \text { China (Jilin) } & \text { UP } \\ & \\ \text { USSR (Primorskiy Kray) } & \text { LP } \\ \text { USSR (Primorskiy Kray) } & \text { LP } \\ \text { China (Heilongjiang) } & \text { UC-LP } \\ \text { USSR (Primorskiy Kray) } & \text { LP } \\ \text { USSR (Primorskiy Kray) } & \text { LP }\end{array}$


C. oblongifolia Radchenko 1966

1976 Durante, pl.70, 4

Mongolia

LP

C. ovifolia Nejburg 1960

Mongolia

UP

C. pentagonata Gorelova 1960

USSR (Primorskiy Kray)

UP

1977 Zimina, pl.17. 13 (aff.)

1978 Zimina, pl.1, 7 (aff.)

C. strelokensis Zimina 1977

1977 Zimina, fig. 23 ; pl.18, 6.10 (all ?)

USSR (Primorskiy Kray)

LP

USSR (Primorskiy Kray)

LP

1978 Zimina, pl.1, 913

USSR (Primorskiy Kray)

$L P$

Remark: in text C.() strelokensis

C. tunguscana Shvedov 1940

1977 Zimina, pl.17, 4.7

Remark: in IFPM. Permian 1971 1975 C.tungushana

USSR (Primorskiy Kray)

LP

\section{Crossotheca Zeiller 1883}

C. sp.

1976 Bohlin, figs.166, 177: pl.12, 12

1977 Schweitzer, fig. 14

1978 Gillespie et al., pl.35, 8A

1978 Schweitzer, fig.3

1978 Strasburger, fig.801A, B

C. crepinii Zeiller 1883

1978 Gillespie et al., pl.35. 8

C. sagittata (Lesquereux 1870) Carpentier 1907

1978 Gillespie et al., pl.35, 10

China (Kansu)

(rec.)

(scheme)

(scheme)

(scheme)

USA (West Virginia)

USA (West Virginia)

(scheme)

\section{Crucicalamites Gothan 1959}

C. sp.

1978 Gillespie et al., fig. $10 \mathrm{H}$

\section{Culmitzschia Ullrich 1964}

C. sp.

1978 Remy et Remy (c), pl.7, 13 15(?)

C. Alorinii Ullrich 1964

1977 Schaumberg, fig. 13

\section{Cupressites Brongniart 1828}

C. hulmannii Brongniart 1828

1885 Quenstedt, pl.98.9. 10

\section{Cycadospadix Schimper 1870}

C. yochelsonii Mamay 1976

1976 Mamay, pl.2. 3

\section{Cyclodendron Kräusel 1928}

C. brasiliensis Lejal-Nicol et Bernardes de Oliveira 1979 1979 Lejal-Nicol et Bernardes de Oliveira, fig.5; pl.1; pl.2: pl.3, 10 (cf.), 1113,14 (cf.), 15 C. leslii (Seward 1903) Kräusel 1928

1980 Retallack, figs.21, 5E, F

\section{Cyclopteris Brongniart 1828}

(. sp).

1976 Barthel (b), pl.2, 10

1976 Barthel et al., pl.33, 4 6; pl.34, 10, 11 (cuti); pl.39, 12 Remark: all of Neuropteris cordata
Brazil (Santa Catarina)

LP

Australia (New South Wales)

L. UP
(C. P)

UC LP

(C.P)

(UC LP?)

(C. P)

(C) P)

UC LP?

$\mathrm{UC} \cdot \mathrm{LP}$ ?

LPSaxo

UPKupf

GFR (Hessen)

$\mathrm{P}$

I.P

GDR (Karl-Marx-Stadt)

LPRotl

GDR (Karl-Marx-Stadt) 
1979 Doubinger et Alvarez-Ramis, pl.2, 3

France (Vosges)

LPAutu

C. rarinervia Goeppert 1864-1865

1976 Barthel et al., pl.30, 8

GDR (Dresden)

LPRotl

\section{Dactylotheca Zeiller 1883}

D. elaverica (Zeiller 1888) Barthel 1975

1976 Barthel (b), pl.1, 3; pl.2, 8

1976 Barthel et al., pl.10, 1-4B, 5 (spore), 6-8B;

pl.11, 1-4, 5-8 (spore)

GDR (Karl-Marx-Stadt)

LPRotl

GDR (Karl-Marx-Stadt)

LPRotl

\section{Dadoxylon Endlicher 1847}

D. sp.

1976 Balasundaram et al., pl.22, 13

1976 Barthel et al., fig.19E; pl.47, 1, 2; pl.48, 1-3

1977 Krumbiegel et Walther, pl.18, 31

1977 Marguerier, pl.1, 1, 5; pl.2, 4

1978 Broutin, pl.1, 1-6

1980 Marguerier et Pacaud, pl.3, 1, 2

D. arberi Seward 1919

1976 Erasmus, pl.1, 1-8

D. malaimbandense Marguerier 1976

1976 Marguerier, figs.1-4; pl.1-5

D. rollei (Unger 1858) Frentzen 1931

1980 Marguerier et Pacaud, pl.1; pl.2, 1, 2

(scheme)

GDR (Karl-Marx-Stadt) LPRotl

GDR (Karl-Marx-Stadt)

LPRotl

France (Saône-et-Loire)

LP

Spain (Sevilla)

$P$

France (Saône-et-Loire)

LPAutu

South Africa (Natal)

UK

Madagascar (Sakamena)

UP-LT

France (Saône-et-Loire)

LPAutu

\section{Damudosorus Pant et Misra 1977}

D. searsolensis Pant et Misra 1977

1977 Pant et Misra, fig.1; pl.1, 1-9; pl.2, 1-7

India (West Bengal)

UPRani

Remark: descr./ills. include cuticles and spores

\section{Damudoxylon Maheshwari 1967}

Remark: in text Damudoxylon (Maheshwari) Maheshwari 1972

See Farr et al. (1979, p.492)

D. iratiensis Guerra-Sommer 1977

1977 Guerra-Sommer, pls.1-3

Brazil (Rio Grande do Sul)

M-UP

\section{Danaeites Göppert 1836}

D. sp.

1980 Tian et Zhang, pl.9, 5(?); pl.10, 2, 5, 7

China (Guizhou)

UP

$D$. emersonii Lesquereux 1880

1978 Gillespie et al., pl.33, 1-7

D. guangdongensis Feng 1977

1977 Feng et al., pl.242, 9

USA (West Virginia)

$\mathrm{UC}-\mathrm{LP}$ ?

$D$. guangxingense Huang 1980

1980 Huang, pl.231, 3-4A

D. mirabilis Gu et Zhi 1974

1978 Zhang, pl.156, 3

1980 Tian et Zhang, pl.11, 1-3, 5

China (Guangdong)

UP

China (Liaoning) LP

China (Guizhou) UP

China (Guizhou) UP

1977 Feng et al., pl.242, 6

D. rigida (Yabe et Oishi 1938) Gu et Zhi 1974

1977 Feng et al., pl.243, 2

1978 Chen et al., pl.152, 6-7; pl.154, 4

China (Guangdong)

UP

China (Guangdong) UP

China (Sichuan) UP

China (Guizhou) UP

D. saraepontanus Stur 1883

1978 Zhang, pl.156, 5

China (Guizhou)

UP 


\section{Denkania Surange et Chandra 1973}

D. indica Surange et Chandra 1973

1977 Chandra et Surange (c), fig.2D; pl.1, 4

1977 Pant, fig.10D

1978 Surange et Chandra, pl.3, 6

1980 Daber (a), fig.1B

India (-)

$P$

(rec. from Surange et Chandra, 1975)

India (Orissa)

(scheme)

\section{Dicalamophyllum Sterzel 1880}

D. altendorfense Sterzel 1880

1976 Barthel et al., pl.28, 11

D. validum Florin 1939

1976 Barthel et al., pl.28, 10; pl.29, 9

GDR (Karl-Marx-Stadt)

LPRotl

GDR (Karl-Marx-Stadt)

LPRotl

Dichotomopteris Maithy 1974

D. sp.

1976 Lele (b), fig.7B

D. ovata Maithy 1977

1977 Maithy (b), fig.3; pl.2, 7

D. qubuensis Hsü 1976

1976 Hsü, fig.3; pl.3, 13-14

(scheme)

India (Bihar)

LGRani?

China (Xizang)

UP

\section{Dicksoniites Sterzel 1881}

Remark: commonly spelled Dicksonites.

See Farr et al. (1979, p.525)

D. pluckenetii (Sternberg 1825) Sterzel 1881

1976 Barthel et al., pl.39, 5-7, 8, 8A

1980 Barthel (c), pl.8, 3, 4

GDR (Karl-Marx-Stadt)

LPRotl

GDR (Gera)

LPRotl

\section{Dicranophyllum Grand'Eury 1877}

D. sp.

1976 Teixeira et Pais, fig.36

1977 Barthel, fig.2, pl.12-14A

D. gallicum Grand'Eury 1877

1976 Barthel et al., pl.42, 1, 2

1976 Bohlin, fig.661

1977 Barthel, fig. 1

1977 Barthel, pls.2, 3, 8, 9

1977 Barthel, pls.4 7

1977 Barthel, pl.10

1977 Barthel, pl.11

1977 Barthel, pl.15

1977 Barthel, pls.16 18

1977 Remy et Remy, fig.47A-C

D. hallei Remy et Remy 1959

1977 Remy et Remy, fig.48

D. striatum Grand'Eury 1877

1977 Barthel, pls.19, 20

(rec. from Jongmans, 1949)
GDR (Karl-Marx-Stadt)
GDR (Dresden)
(from Renault et Zeiller, 1888)
(rec.)
GDR (Gera)
GDR (Suhl)
GDR (Karl-Marx-Stadt)
GDR (Dresden)
France (Loire)
France (Saône-et-Loire)
GDR (Suhl)
GDR (Suhl)
France (Allier)

(C-P)

LPRotl

LPRotl

LPAutu

LPRotl

LPRotl

LPRotl

UCStep-LPAutu

UCStep-LPAutu

LPAutu

LPAutu

UCStep--LPAutu

\section{Dictyopteridium Feistmantel 1881}

D. sp.

1976 Balasundaram et al., pl.22, 11

1976 Benecke, fig. 2

D. feistmantelii Chandra et Surange 1976

1976 Chandra et Surange, figs. $1-3,5,6$; pls. $1-8$

1976 Chandra et Surange, fig. 7

Remark: descr./ills. include cuticles.

Organic connection with Glossopteris tenuinervis

(scheme)

(scheme)

India (West Bengal)

(rec.)
UPRani 
1977 Chandra et Surange (c), fig.2F

1977 Chandra et Surange (e); fig.14A

Remark: organic connection with Glossopteris tenuinervis

1978 Surange et Chandra, pl.1, 1

1979 Surange et Chandra, pl.14, 6; pl.22, 13

Remark: organic connection with Glossopteris tenuinervis

D. flabellatum Benecke 1976

1976 Benecke, fig.2

1976 Benecke, figs. 3, 7-24, 88

D. sporiferum Feistmantel 1881

1976 Benecke, figs.1, 4-6

Remark: fig. 4 possible association with

Glossopteris. Figs.5, 6 organic connection with seeds

1976 Schopf, fig.9B

1977 Pant, fig.10A

1978 White, figs.55-57

Remark: organic connection with seeds

1979 Banerjee, pl.51, 20-22

Remark: organic connection with

Glossopteris browniana

\section{Diphyllopteris Srivastava 1978}

D. verticillata Srivastava 1978

1978 Srivastava, fig.1; pl.1, 1-3

Diplocalamites Gothan 1959

$D$. sp.

1978 Gillespie et al., fig.10G

\section{Discinites Feistmantel 1879}

D. orientalis $\mathrm{Gu}$ et $\mathrm{Zhi} 1974$

1980 Zhao et al., pl.1, 5-5A (cf.)

\section{Distichotheca Gu et Zhi 1974}

D. crossothecoides Gu et Zhi 1974

1980 Zhao et al., pl.22, 6-8A

Dizeugotheca Archangelsky et De la Sota 1961

D. sp.

1976 Lele (b), fig.7C

1977 De la Peña et al., pl.1, 6

1978 Rösler, pl.1, 2-6(?)

D. bortoluzzii Cazzulo-Klepzig 1978

1978 Cazzulo-Klepzig, fig.5A-D; pl.1, 5, 6; pl.2, 3, 4

Remark: in text fig.5; pl.5, 1-2; pl.6, 1-2

D. qubuensis Hsü 1976

1976 Hsü, fig. $2 ;$ pl.2, 8-12

1978. Hsü, pl.2, 3-6

\section{Dolerotheca Halle 1933}

+ D. pennsylvanicum (Dawson 1890). White

1978 Gillespie et al., pl.37, 1, 2, 5, 11

\section{Dolianitia Millan 1967}

D. crassa Millan 1967

1977 Pant, fig.6H, I (scheme)

(rec.)

India (West Bengal)

LGRani

(rec.)

South Africa (Natal) UP

South Africa (Natal) UP

India (Orissa) $P$

(rec. from Surange et Chandra, 1975)

Australia (New South Wales) (U)P

India (West Bengal) UPRani

India (Bihar) LPBara

(scheme)

China (Guizhou/Yunnan)

UP

China (Guizhou/Yunnan)

UP

(scheme)

Spain (Soria) LPAutu

Brazil (Paraná) UP

Brazil (Santa Catarina) UPKaza-Tata

China (Xizang) UP

China (Xizang) UP

USA (West Virginia) UC-LP?

(from Millan, 1957) 
D. madagascariensis Appert 1977

1977 Appert, figs.5 10; pl.36, 1, 2; pl.37, 7-9; pl.38-40

Remark: with seeds Cordaicarpus madagascariensis in organic connection

\section{Dorycordaites Zeiller 1888}

D. sp.

1977 Krumbiegel et Walther, fig.2.100.3

(rec. from Grand'Eury)

\section{Dunedoonia Holmes 1977}

D. reticulata Holmes 1977

1977 Holmes, fig.1; pl.1

Australia (New South Wales) U?P

\section{Dzungariella Sal'menova 1979}

D. fucoides Sal'menova 1979

1979 Sal'menova, pl.13, 1-3

D. kizilkainarica Sal'menova 1979

1979 Sal'menova, pl.13, 4

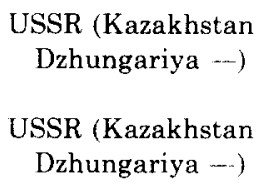

UP

UP

\section{+ Eichwaldia Zalessky 1927}

Remark: non Eichwaldia Ledebour 1833. See Farr et al. (1979:599)

E. sp.

1976 Durante, pl.24, 36

\section{Elatra Appert 1977}

E. bella Appert 1977

1977 Appert, pl.32, 14

Madagascar (Sakoa Basin)

LGKarh-Bara?

\section{Emplectopteridium Kawasaki 1934}

E. alatum Kawasaki 1934

1976 Asama, fig.1(5)

1976 Asama, pl.29, 2

1977 Feng et al., pl.245, 5

1979 Asama, fig.3(5)

1980 Asama, fig.5

Mongolia

UP

\section{Emplectopteris Halle 1927}

\section{E. sp.}

1976 Bohlin, fig.B (p.44)

1979 Asama, fig.5 (1 4)

1979 Yuasa, fig.3.7

E. neimongolensis Huang 1976

1976 Huang, pl.209, 1, 2

E. sinensis Huang 1976

1976 Huang, pl.220, 1 5

E. triangularis Halle 1927

1976 Asama, fig.1(1)

1976 Asama, pl.28, 1, 1A

1976 Asama, pl.28, 1B

1976 Huang, pl.221, 3

1977 Feng et al., pl.244, 1, 2

1977 Remy et Remy, fig.35I

1977 Schweitzer, fig. 19E

1979 Asama, fig.2D (scheme)

South Korea (Seizen) UP

China (Henan) LP

(scheme)

(C?-P) (scheme)

(scheme)

(scheme from Halle, 1931)

China (Inner Mongolia)

China (Inner Mongolia)

(scheme)

China (Shanxi) LP

China (-)

China (Inner Mongolia)

China (Henan)

China (Shanxi)

(rec.)

China (Shanxi)
(UC $\cdot$ LP)

(C-P)

L.P

LP

LP

LP

LP

LP

(C P)

LP 
1979 Asama, figs.3(1), 4D

1979 Asama, fig.17E

1980 Asama, fig.5A

\section{Equisetites Sternberg 1833}

E. sp.

1976 Bohlin, fig.9(?)

E. elongatus Fontaine et White 1880

1977 De la Peña et al., pl.1, 7

\section{Equisetum Linnaeus 1753}

E. sp.

1976 Durante, pl.18, 5

1976 Gordon-Gray et al., pls.1-4(?)

\section{Eremopteris Schimper 1869}

$E$. crenulata Lesquereux 1876

1978 Gillespie et al., pl.52, 4

E. missouriensis Lesquereux 1880

1978 Gillespie et al., pl.51, 4

\section{Eretmonia Du Toit 1932}

$E . \mathrm{sp}$.

1976 Schopf, fig.10; pl.1, 5

1976 Schopf, pl.4, 1-6

1977 Chandra et Surange (d), figs.1E-I, 2, (cuti), 4 (cuti); pl.1, 1-2 (cuti); pl.2, 11-12 (cuti)

1977 Pant, fig.8C

1978 White, figs.7 (type), 50, 51

1978 White, fig.54

Remark: organic connection with sporangial cluster 1980 Daber (a), fig.1E

E. cooyalensis Holmes 1974

1976 Gould, fig.4C

1976 Schopf, fig.9C

1980 Retallack, fig. $21.5 \mathrm{~K}$

E. emarginata (Surange 1957) Chandra et Surange 1977

1977 Chandra et Surange (c), fig.1G, H; pl.1, 2

1978 Surange et Chandra, pl.1, 4

E. hinjridaensis Surange et Maheshwari 1970

1977 Chandra et Surange (c), fig.1F

1978 Surange et Chandra, pl.1, 3

E. karanpurensis Surange et Maheshwari 1970

1978 Surange et Chandra, pl.2, 2

E. natalensis Du Toit 1932

1978 White, figs.45-49

1978 White, fig. 54

Remark: organic connection with sporangial cluster(s) 1979 Van Dijk et al., pl.46, 23-25

$+E$. ovoides Surange et Chandra 1974

Remark: in IFPM. Permian 1971-1975 p.20: E. ovata 1977 Chandra et Surange (c), fig.1D; pl.1, 6

1978 Surange et Chandra, pl.3, 2

E. utkalensis Surange et Maheshwari 1970

1976 Schopf, fig.9D

1977 Chandra et Surange (c), fig.1E

1978 Surange et Chandra, pl.3, 3 (scheme)

China (Shanxi)

$\mathrm{P}$

(scheme)

\author{
China (Kansu)
}

UC-LP

Spain (Soria)

LPAutu

Mongolia

UP

South Africa (Natal)

$\mathrm{P}$
UC-LP?

USA (West Virginia) UC-LP?

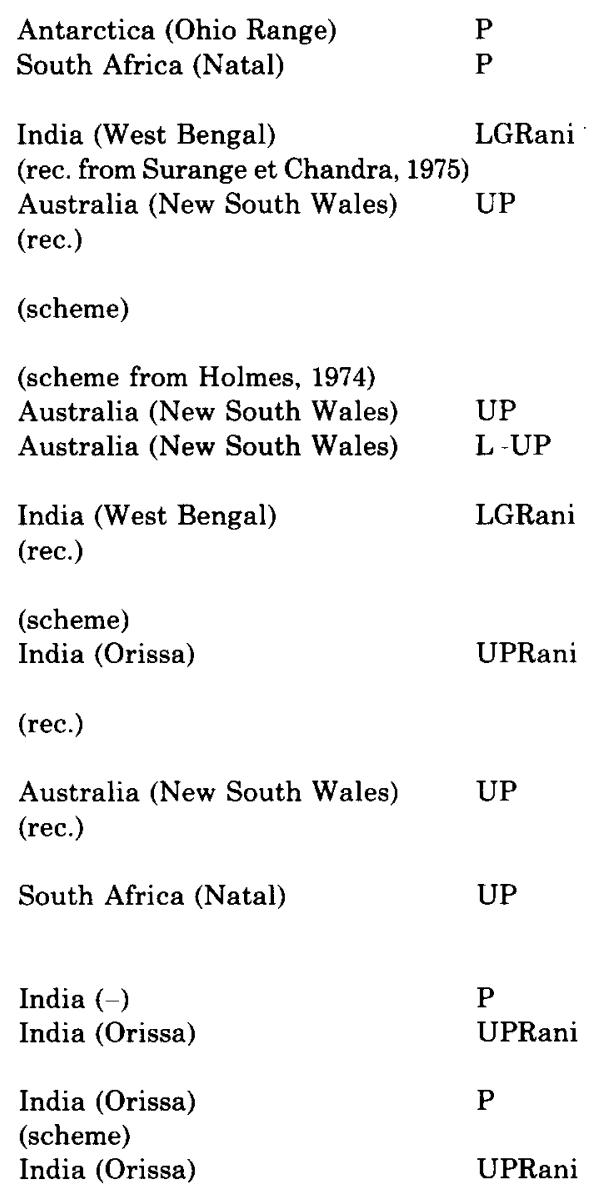


Ernestiodendron Florin 1934

$E$. sp

1976 Grzimek, fig.8.25B

1979 Asama, fig. $12 \mathrm{G}$

E. filiciformia (Schlotheim ex Sternberg 1825) Florin 1934

Remark: commonly spelled $E$. filiciforme

1976 Barthel et al., pl.41, 1

1977 Krumbiegel et Walther, fig.2.101.2

1977 Tenchov (b), pl.3, 6

Remark: in text E. filiciformis

1978 Doubinger et al., pl.1, 2; pl.2, 6

1978 Grauvogel-Stamm, fig.44D

1978 Miller, fig.1C

(scheme)

(scheme from Florin, 1944)

GDR (Karl-Marx-Stadt)

LPRotl

GDR (Karl-Marx-Stadt) LPRotl

Bulgaria (Northwest)

Spain (Gerona)

LPAutu (scheme from Schweitzer, 1963) (UC-P)

\section{Etapteris Bertrand 1907}

E. lacattei (Renault 1869) Bertrand 1907

1978 Galtier, pl.1, 1

France (Saône-et-Loire)

LPAutu

\section{Eucordaites Grand'Eury 1890}

E. sp.

1977 Krumbiegel et Walther, fig.2.100.2

(rec. from Grand'Eury)

\section{Europhyllites Glukhova 1978}

Remark: type specimen is Cordaites crassus

(Renault 1879 as figured by Harms et Leisman (1963)

from the Pennsylvanian of USA (Iowa).

\section{Euryphyllum Feistmantel 1879}

E. sp.

1976 Lele (b), fig.2

E. whittianum Feistmantel 1879

1977 Srivastava (a), fig. $7 ;$ pl.1, 4

1979 Leguizamon, pl.1, 1-5

(scheme)

India (Bihar)

LGKarh

Argentina (San Luis)

LP

\section{Eusphenopteris (Schimper 1879) Kidston 1882}

E. obtusiloba (Brongniart 1829) Novik 1952

1978 Gillespie et al., pl.52, 7

E. striata (Gothan 1913) Novik 1952

1978 Gillespie et al., pl.52. 1

(scheme)

(UC . LP?)

USA (West Virginia)

$\mathrm{UC}$ LP?

\section{Fascipteridium Zhang et Mo 1979}

F. ellipticum Zhang et Mo 1979

1979 Zhang et Mo, pl.3, 1 5

China (Henan)

UP

\section{Fascipteris Gu et Zhi 1974}

$F$. densata Gu et Zhi 1974

1976 Huang, pl.222, 4

1978 Zhang, pl.156, 1

1980 Zhao et al., pl.12, 7, 8

F. hallei (Kawasaki 1931) Gu et Zhi 1974

1976 Huang, pl.222, 13

1977 Feng et al., pl.242, 3

1980 Huang, pl.238, 5, 5A

1980 Zhao et al., pl.12, 9

China (Inner Mongolia)

China (Guizhou)

China (Guizhou/Yunnan)

China (Inner Mongolia)

China (Guangdong)

China (Liaoning)

China (Guizhou/Yunnan)
UP

UP

UP

UP

UP

UP

UP 
F. kaishantunensis Huang 1980

1980 Huang, fig.27; pl.234, 1-5

$F$. robusta (Kawasaki 1934) Huang 1980

1980 Huang, pl.234, 6-7

$F$. sinensis (Stockmans et Mathieu 1939) Gu et Zhi 1974

1980 Huang, pl.235, 1-4

F. stena $\mathrm{Gu}$ et Zhi 1974

1977 Feng et al., pl.242, 7, 8

1978 Zhang, pl.156, 4

1980 Tian et Zhang, fig.19; pl.10, 3, 3A, 6, 8, 8A; pl.11, 7

1980 Zhao et al., pl.12, 10, 10A

$\begin{array}{lc}\text { China (Jilin) } & \text { UP } \\ \text { China (Jilin) } & \text { UP } \\ \text { China (Liaoning) } & \text { UP } \\ & \\ \text { China (Guangdong) } & \text { UP } \\ \text { China (Guizhou) } & \text { UP } \\ \text { China (Guizhou) } & \text { UP } \\ \text { China (Guizhou/Yunnan) } & \text { UP }\end{array}$

\section{Fertiliger Schopf 1976}

F. sp.

Remark: not proposed by Schopf (1976) as genus name

1980 Cazzulo-Klepzig et al. (a), pl.3, 16

Brazil (Rio Grande do Sul)

LPSakm

Brazil (Rio Grande do Sul)

LPSakm

1980 Millan et Dolianiti (a), fig.1; pl.1, 1-3 (all"")

Brazil (São Paulo)

LP

\section{Fetura Benecke 1976}

F. natalensis (Lacey, Van Dijk et Gordon-Gray 1975) Benecke 1976

1976 Benecke, fig.3

1976 Benecke, figs.25-41

(rec.)

South Africa (Natal)

Remark: fig.34 association with Glossopteris

\section{Filicites Schlotheim ex Brongniart 1822}

F. aquilinus Schlotheim 1804

1885 Quenstedt, pl.91, 16

GDR (Gera)

LP

\section{Gamophyllites Radchenko 1962}

G. sp

1977 De la Peña et al., pl.1, 8

Spain (Soria)

LPAutu

\section{Gangamopteris McCoy 1875}

$G$. sp.

1976 Anderson et McLachlan, pl.1, 5

1976 Balasundaram et al., pl.22, 7

1976 Lele (b), figs.1, 2

1976 Lele (b), fig. 3

1977 Appert, pl.4, 10(?); pl.9, 4-5 (cf.)

1977 Zimina, pl.4, 6

1980 Bohlin, pl.1, 5, 7-13, 16, 18, 19, 21

1980 Bohlin, pl.2, 1-20

1980 Cazzulo-Klepzig et al. (a), pl.2, 3; pl.3, 8, 11

1980 Guerra-Sommer et al., pl.1, 5

G. angustifolia (McCoy 1847) McCoy 1875

1977 Correa da Silva et Arrondo, pl.1, 4

1977 Maithy (c), pl.1, 3

1978 Correa da Silva, pl.5, 6

1980 Cazzulo-Klepzig et al. (a), pl.1, 8; pl.3, 14

1980 Cazzulo-Klepzig et al. (b), pl.2, 1, 10

G. buriadica Feistmantel 1879

1977 Correa da Silva et Arrondo, pl.1, 5

1978 Correa da Silva, pl.5, 3

1980 Cazzulo-Klepzig et al. (a), pl.1, 1, 2, 4, 5, 7

1980 Cazzulo-Klepzig et al. (b), pl.2, 11

\begin{tabular}{ll}
$\begin{array}{l}\text { South Africa (Cape Province) } \\
\text { (scheme) }\end{array}$ & K \\
(scheme) \\
(rec. from Plumstead, 1966) \\
$\begin{array}{l}\text { Madagascar (Sakoa Basin) } \\
\text { USSR (Primorskiy Kray) } \\
\text { (scheme) }\end{array}$ & LGKarh-Bara? \\
$\begin{array}{l}\text { Australia (-) } \\
\text { Brazil (Rio Grande do Sul) } \\
\text { Brazil (Rio Grande do Sul) }\end{array}$ & UP \\
& LPSakm \\
Brazil (Rio Grande do Sul) & LPSakm-Arti \\
$\begin{array}{l}\text { India (Bihar) } \\
\text { Brazil (Rio Grande do Sul) } \\
\text { Brazil (Rio Grande do Sul) } \\
\text { Brazil (Rio Grande do Sul) }\end{array}$ & $\begin{array}{l}\text { LGKarh } \\
\text { LPSakm }\end{array}$ \\
$\begin{array}{l}\text { Brazil (Rio Grande do Sul) } \\
\text { Brazil (Rio Grande do Sul) } \\
\text { Brazil (Rio Grande do Sul) } \\
\text { Brazil (Rio Grande do Sul) }\end{array}$ & LPSakm \\
\hline
\end{tabular}


G. clarkeana Feistmantel 1878

1977 Srivastava (a), fig. $3 ;$ pl.2, 9

G. cyclopteroides Feistmantel 1876

1976 Gould, fig. $3 \mathrm{H}, \mathrm{I}$

1977 Appert, pl.1, 3 5; pls.2 3; pl.4, 1, 2, $3.9(?)$

1977 Srivastava (a), fig. 2 ; pl.1, 1,2

1977 Zimina, pl.3, 1 4; pl.4, 1-2 (all aff.)

1980 Retallack, fig.21.3I

G. cyclopteroides Feistmantel 1876

var. attenuata Feistmantel 1879

1977 Kovács-Endrödy, pl.4, 1, 2

G. major Feistmantel 1879

1980 Retallack, fig.21.3J

G. oblanceolata Maithy 1970

1977 Appert, pl.5, 12 (cf.)

G. obovata (Carruthers 1869) White 1908

1977 Correa da Silva et Arrondo, pl.1, 3

1980 Cazzulo-Klepzig et al. (a), pl.1, 6 ; pl.3, 9

1980 Cazzulo-Klepzig et al. (b), pl.1, 2, 14

G. pacifica Zimina 1967

1977 Zimina, fig. 11: pl.2, 6

G. spathulata McCoy 1875

1980 Retallack, fig. $21.3 \mathrm{H}$

G. ussuriensis Zimina 1967

1977 Zimina, fig.12; pl.2, 7

$\begin{array}{ll}\text { India (Bihar) } & \text { LGKarh } \\ \begin{array}{l}\text { (scheme) } \\ \text { Madagascar (Sakoa Basin) }\end{array} & \begin{array}{l}\text { LGTalc--Karh? } \\ \text { LGKia (Bihar) }\end{array} \\ \begin{array}{l}\text { USSR (Primorskiy Kray) } \\ \text { Australia (New South Wales) }\end{array} & \text { UP UP } \\ & \\ \text { (from Feistmantel, 1879) } & \\ \text { Australia (New South Wales) } & \text { L-UP } \\ \text { Madagascar (Sakoa Basin) } & \text { LGTale Karh? } \\ \text { Brazil (Rio Grande do Sul) } & \text { LPSakm Arti } \\ \text { Brazil (Rio Grande do Sul) } & \text { LPSakm } \\ \text { Brazil (Rio Grande do Sul) } & \text { LPSakm } \\ \text { USSR (Primorskiy Kray) } & \text { UP } \\ \text { Australia (New South Wales) } & \text { L-UP } \\ \text { USSR (Primorskiy Kray) } & \text { UP }\end{array}$

\section{Gansucladus Bohlin 1976}

G. parvifolia Bohlin 1976

1976 Bohlin, figs.251 254; pl.14, 3, 4; pl.16, 2 7;

pl.27. 48

\section{Gaussia Nejburg 1934}

Remark: non Gaussia Wendland 1865.

See Farr et al. (1979, p.704)

G. scutellata Nejburg 1934

1977 Zimina, pl.22, 4

\section{Gigantonoclea Koidzumi 1936}

G. sp.

1977 Feng et al., fig. 107, 3

1977 Feng et al., pl.247, 6(?)

1977 Feng et al., pl.248, 3-4

G. acuminatiloba (Shimakura 1940) Gu et Zhi 1974

1977 Feng et al., pl.247. 1, 2

G. dictyophylloides $\mathrm{Gu}$ et Zhi 1974

1978 Zhang, pl.160, 3

G. guizhouensis Gu et Zhi 1974

1978 Chen et al., pl.152, 8

1978 Zhang, pl.160, 1 (cf.), 2

1980 Tian et Zhang, fig.20; pl.11, 4; pl.13, 2

1980 Zhao et al., pl.16, 56

G. hallei (Asama 1959) Gu et Zhi 1974

1978 Zhang, pl.160, 4

1980 Zhao et al., pl.15, 5; pl.20, 4

G. lagrelii (Halle 1927) Koidzumi 1936

1976 Asama, fig.1(2)

1976 Asama, pl.28, 2, 2A

1977 De la Peña et al., pl.2, 1, 2

1977 Feng et al., figs.107. 2; 108
USSR (Primorskiy Kray)

LP

$\begin{array}{ll}\begin{array}{ll}\text { (scheme) } \\ \text { China (Guangxi) } \\ \text { China (Hunan) }\end{array} & \text { UP } \\ \text { UP } & \\ \text { China (Guangdong) } & \text { UP } \\ \text { China (Guizhou) } & \text { UP } \\ \text { China (Sichuan) } & \text { UP } \\ \text { China (Guizhou) } & \text { UP } \\ \text { China (Guizhou) } & \text { UP } \\ \text { China (Guizhou/Yunnan) } & \text { UP } \\ & \\ \text { China (Guizhou) } & \text { UP } \\ \text { China (Guizhou/Yunnan) } & \text { UP } \\ & \\ \text { (scheme) } & \\ \text { China (Shanxi) } \\ \text { Spain (Soria) } \\ \text { (scheme) }\end{array}$

(scheme) 
1977 Feng et al., pl.247, 3

1978 Zhang, pl.159, 4

1979 Asama, fig.2C

1979 Asama, figs.3(2), 4C

1980 Asama, fig.5

1980 Zhang, pl.1, 1, 2

1980 Zhao et al., pl.17, 7

G. longifolia (Kodaira 1930) Gu et Zhi 1974

1978 Zhang, pl.160, 5(?)

G. microphylla Huang 1980

1980 Huang, pl.239, 1-7

G. minor Feng 1977

1977 Feng et al., pl.246, 4

G. plumosa Mo 1980

1980 Zhao et al., pl.16, 1-3

G. rosulata $\mathrm{Gu}$ et $\mathrm{Zhi} 1974$

1977 Feng et al., pl.247, 4, 5

1980 Tian et Zhang, pl.12, 2, 4; pl.13, 1

G. tieyingensis Huang 1980

1980 Huang, fig.38; pl.238, 1-4

G. unita Huang 1980

1980 Huang, fig.39; pl.236, 1-5

+ G. yabei (Kawasaki)

1977 Feng et al., pl.248, 1-2(?)

G. yujiaensis Huang 1980

1980 Huang, fig.37; pl.237, 1-3

\section{Gigantopteridium Koidzumi 1934}

G. americanum (White 1912) Koidzumi 1934

1976 Asama, pl.30, 1

\section{Gigantopteris Schenk 1883}

G. sp.

1976 Asama, pl.30, 2, 3

1976 Lele (a), fig.4

1977 Feng et al., pl.107, 4, 5

1979 Yuasa, fig.3.8

G. cordata Yabe et Oishi 1938

1980 Tian et Zhang, pl.15, 2

G. dictyophylloides $\mathrm{Gu}$ et $\mathrm{Zhi} 1974$

1977 Feng et al., pl.246, 3

1978 Chen et al., pl.153, 1-2

1980 Tian et Zhang, fig.21; pl.16, 1, 1A, 2, 2A

1980 Zhao et al., pl.16, 4

G. meganetes Tian 1980

1980 Tian et Zhang, fig.21; pl.17, 1, 3; pl.18, 2, 2A

G. nicotianaefolia Schenk 1883

1976 Asama, fig.1(7)

1976 Asama, pl.29, 4, 4A

1977 Feng et al., pl.246, 1 ; pl.253, 4

1978 Chen et al., pl.153, 3

1979 Asama, fig.3(7)

1980 Asama, fig.5

1980 Tian et Zhang, pl.15, 1, 1A, 1B

G. paradoxa Mo 1980

1980 Zhao et al., pl.15, 1-4

\section{Gigantospermum Jongmans et Gothan 1935}

G. sp.

1980 Tian et Zhang, figs.25, 26; pl. 4, 6, 8; pl.5, 6, 10; pl.19, 5, 6; pl.21, 4, 5; pl.22, 1, 10, 13
USA (Texas)

LP

China (Henan) UP

China (Guizhou) UP

China (Shanxi) UP

(scheme)

(scheme)

China (-)

China (Guizhou/Yunnan)

$P$

China (Guizhou) UP

China (Liaoning) UP

China (Guangdong) UP

China (Guizhou/Yunnan) UP

China (Guizhou) UP

China (Liaoning) UP

China (Liaoning) UP

China (Hunan) UP

China (Liaoning) LP

\begin{tabular}{|c|c|}
\hline $\begin{array}{l}\text { USA (Texas) } \\
\text { (scheme) } \\
\text { (scheme) } \\
\text { (scheme from Asama, 1959) }\end{array}$ & $(\mathrm{C}-\mathrm{P})$ \\
\hline China (Guizhou) & UP \\
\hline China (Guangdong) & UP \\
\hline China (Sichuan) & UP \\
\hline China (Guizhou) & UP \\
\hline China (Guizhou/Yunnan) & UP \\
\hline China (Guizhou) & UP \\
\hline (scheme) & \\
\hline North Korea (Tokusen) & LP \\
\hline China (Hunan) & UP \\
\hline $\begin{array}{l}\text { China (Sichuan) } \\
\text { (scheme) } \\
\text { (scheme) }\end{array}$ & UP \\
\hline China (Guizhou) & UP \\
\hline China (Guizhou/Yunnan) & UP \\
\hline
\end{tabular}

China (Guizhou)

UP
China (Henan) UP 


\section{Ginkgo Linnaeus 1771}

G. sp.

1979 Lichter, figs.3(7), 4

(rec.)

\section{Ginkgoites Seward 1919}

$G$. sp.

1976 Durante, pl.51, 2, 3; pl.52, 4

1977 Broutin, fig. 1; pl.2, 3

G. xinhuaensis Feng 1977

1977 Feng et al., pl.2449, 5

Mongolia

Spain (Sevilla)

China (Henan)

Spain (Sevilla)

China (Heilongjiang)

Brazil (Rio Grande do Sul)

Brazil (Rio Grande do Sul)

Brazil (Rio Grande do Sul)

Brazil (Rio Grande do Sul)

(scheme)

China (Guangdong)
UP

UCStep?-LPAutu?

UP

UCStep?--LPAutu?

$\mathrm{UP}$

LPSakm-Arti

LPSakm

LPSakm

LPSakm

UP

\section{+ Ginkgophyton Zalessky 1919}

Remark: non Ginkgophyton Matthew 1910.

Substitute name: Ginkgophytopsis Hoeg 1967

G. giganteum Burago 1976

1976 Burago, figs.1, 2

USSR (Primorskiy Kray)

UP

Remark: in Paleontol. Zh., 1977(1): 142 as new combination Ginkgophytopsis gigantea (Burago 1976) Burago 1977

+ G. komalarjunii (Asama 1966)

1976 Burago, fig.1

+ G. maiyaense (Asama 1967)

1976 Burago, fig. 1

Thailand ( )

Japan (-)

China (Xizang)

China (Heilongjiang)

UP

\section{Glossopteris Brongniart 1828}

$G$. sp.

1976 Anderson et McLachlan, pl.1, 7 -9

1976 Anderson et McLachlan, pl.2, 14

1976 Balasundaram et al., pl.22, 6

1976 Bohlin, figs.303 (aff.), 650 (cf., fruct.); pl.30,

$12 \cdot 14$ (cf.)

1976 Bohlin, figs.558 (aff.), 698 (cf., fruct.), 699 (aff.)

$\begin{array}{ll}\begin{array}{l}\text { South Africa (Cape Province) } \\ \text { Namibia (Gellap Ost) } \\ \text { (scheme) }\end{array} & \text { LKEcca } \\ & \text { K } \\ \text { (LP -LT) } \\ \text { China (Chinghai) } & \text { UC - LP } \\ \text { China (Kansu) } & \text { UC - - LP }\end{array}$


1976 Daber, fig.479(1)

1976 Grzimek, fig.p.52

1976 Lele (a), fig.4

1976 Lele (b), figs.1, 2

1976 Lele (b), fig.3

1976 Schopf, pl.5, 5, 6

1976 Teixeira et Pais, fig.35C, D

1977 Appert, pl.5, 3, 4

1977 Appert, pl.9, 1-3; pl.25, 1; pl.28, 5, 6; pl.30,

4,$5 ;$ pl.31, 1-12

1977 Bose et al., fig.5; pl.1, 10; pl.2, 19, 20

1977 Chandra et Surange (b), figs.1, 2, 3-4 (cuti);

pl.1, 1-4, 5 (cuti); fig. 8 (rec.)

Remark: organic connection with Venustostrobus indicus 1977 Gould et Delevoryas, fig.1

1977 Gould and Delevoryas, figs.2A-G, 4A-I, 5A, B, 6A-J, 7A-E

Remark: descr./ills. include anatomy. Association with female fructifications and Arberiella; seed with bisaccate pollen grains

1977 Kovács-Endrödy (b), fig.4

1977 Kovács-Endrödy (b), pl.5, 1, 2; pl.9, 2, 3; pl.10, 1-3; pl.13, 3, 4;

pl.14, 1, 2; pl.16, 2; pl.17, 1-3; pl.18, 1-3

1977 Minato, pl.4-1, 1, 2, 4; pl.4-2, 3, 4

1977 Pant, fig.1E-H (cuti)

1977 Pant, fig.2A

1977 Pant, fig.2B

1977 Pant, fig.5

1977 Pant, fig.10E

Remark: fig.10E organic connection with Ottokaria

1977 Schweitzer, fig. $7\left(7,7^{\prime}\right)$

1977 Wesley, fig. p.210

1977 Zimina, pl.4, 9; pl.9, 1 (all ?)

1978 Craddock, fig.2

1978 Jeyasingh, figs.1-2 (cuti)

1978 Strasburger, fig. $800 \mathrm{C}$

1978 Waterhouse, fig.11.4

1979 Kovács-Endrödy, figs.2.1, 3.1

1979 Kovács-Endrödy, pl.2,A; pl.11,A, B

1979 Kovács-Endrödy, pl.2,C

1979 Rösler, fig.4(3)

1979 Scheven, fig.40

1979 Srivastava, fig. $9 ;$ pl.1, 4, 5

1979 Steel et Harvey, fig.p.121

1979 Yuasa, fig.3.12

1980 Andrews, fig. p.242

1980 Boersma, fig.2

1980 Cazzulo-Klepzig et al. (a), pl.2, 2, 5

1980 Gould et Shibaoka, fig.6A

1980 Gould et Shibaoka, fig.6B

1980 Guerra-Sommer et al., pl.12, 9

G. acuta Dun 1897

1978 Banerjee (a), fig.21; pl.9, 21

G. ampla Dana 1849

1976 Kovács-Endrödy, figs.3, 13, 20; pl.1, A

1976 Mildenhall, figs.1, 2

1977 Kovács-Endrödy (b), pl.19, 1-3

1977 Srivastava (b), fig.6; pl.3, 15 (all cf.)

1978 White, fig.30

Remark: association with Squamella ampla (rec.)

South Africa (-) C C-P

(scheme)

(scheme)

(rec. from Pant et Singh, 1974)

Antarctica (Queen Alexandra

Range)

(scheme from Pant, 1962)

Madagascar (Sakoa Basin)

Madagascar (Sakoa Basin)

India (Madhya Pradesh)

$\mathrm{P}$

LGTalc-Karh?

LGKarh-Bara?

UP-LT

India (West Bengal)

LGRani

(rec.)

Australia (Queensland)

UP

(scheme)

South Africa (Transvaal) $\quad P$

Chile (Valdivia)

UC-L.P?

(from Pant et Singh, 1971)

(rec. from Pant et Singh, 1974)

(rec. from Rigby, 1969)

(scheme; anatomy)

(rec. from Pant et Nautiyal)

(rec.)

(scheme)

USSR (Primorskiy Kray) UP

Antarctica (Ellsworth Mts.) C-P

India (Bihar) LG

(scheme from Gothan) (C-P)

New Zealand (South Island) P

(scheme)

South Africa (Transvaal) $P$

South Africa (Orange Free State) $P$

(scheme)

Rhodesia

India (Bihar)

(scheme)

(scheme from Plumstead, 1956)

Antarctica (Coalsack Bluff) P

(scheme)

Brazil (Rio Grande do Sul)

(scheme from Raven, 1972)

(scheme)

Brazil (Rio Grande do Sul)

LPSakm

Australia (New South Wales) UP

South Africa (Transvaal) P

New Zealand (South Island) UPKaza

South Africa (Transvaal) P

India (Bihar)

PGBara

Australia (New South Wales) UP 
1979 Kovács-Endrödy, figs.3.5, 3.6; pl.3, A; pl.4, B;

pl.5, A; pl.6, A, B: pl.7. A, B; pl.9, A. B

1980 Rigby et al., figs.13, 14

G. andreanskyi Kovács-Endrödy 1977

1977 Kovács-Endrödy (a), figs. $1 \cdots 4$

Remark: new name for $G$. pseudocommunis Kovács-Endrödy 1976. a later homonym of $G$. pseudocommunis Pant et Gupta 1968

G. angusta Pant et Gupta 1971

1979 Chandra et Surange, figs.12B, 27F, G, 44C, 49R:

pl.1, 9; pl.6. 7 ; pl.17. 3, 8

1979 Chandra et Surange, pl.43, 7

G. anguslifolia Brongniart 1830

1976 Hsü, pl.3, 16, 17

1977 Bose et al., pl.1. 3-6

1978 Banerjee (a). fig. 1; pl.1, 1

1978 Hsü, pl.1, 5,6

1978 Sarkar. figs. 1, 2

1978 Sarkar, fig.3

1979 Chandra et Surange, figs.23B F, 25B, 25C, E, 46.J, M, N, S; pl.3, 6; pl.13, 5; pl.18, 7,11

1979 Chandra et Surange, fig.23G

1979 Chandra et Surange, fig. $23 \mathrm{H}$

1979 Chandra et Surange, figs.24D, 46P

1979 Chandra et Surange, pl.42, 2

1980 Rigby et al., figs.15-17. 19

G. arberi Srivastava 1956

1979 Chandra et Surange, figs.4D, 10A, 18A, 45G, H, 48F; pl.7.

4. $7 ; \mathrm{pl} .8,6 ; \mathrm{pl} .16,4.9 ; \mathrm{pl} .17 .2$

1979 Chandra et Surange, pl.40, 1

G. barakarensis Kulkarni 1971

1977 Srivastava (b), fig.2; pl.2, 11

1979 Chandra et Surange, figs.5B, 19G, I, 48E, I; pl.2, 4; pl.19, 7

1979 Chandra et Surange, pl.41, 3

$G$. bosei Chandra et Surange 1979

1979 Chandra et Surange, fig.11C

1979 Chandra et Surange, figs.11G, 12D, E, G, 22E, 45D, 46E, F, $53 \mathrm{I}$

1979 Chandra et Surange, pl.47, 5

G. brongniartii Pant et Gupta 1968

1979 Chandra et Surange, fig.21G; pl.23, 3. 16

G. brouniana Brongniart 1830

1885 Quenstedt, pl.92,10

1976 Gould, fig. $3 \mathrm{~F}$

1976 Kovács-Endrödy, fig.1A

1976 Kovács-Endrödy, figs.1B, 5; pl.4, E

1976 Lele (b), fig. $\bar{x}$

Remark: in text $G$. brownii

1977 Appert, pl.29, 46

1977 Kovács-Endrödy (b), fig.1

1977 Kovács-Endrödy (b), pl.14, 3; pl.16, 1

1977 Srivastava (b), pl.2, 12

1978 Sarkar, fig. 4

1978 Sarkar, fig. 5

1979 Banerjee, pl.51, 15, 16, 20

Remark: organic connection with Dictyopteridium sporiferum and Scutum leslium

1979 Banerjee et al., pl.36, 2

1979 Chandra et Surange, figs.14B D, 47D

1979 Chandra et Surange, pl.38, 1

South Africa (Transvaal)

$\mathrm{P}$

Australia (New South Wales)

South Africa (Transvaal)

P T?

India (Bihar)

LGKarh

(rec.)

China (Xizang)

UP

India (Madhya Pradesh)

India (West Bengal)

China (Xizang)

(from Feistmantel, 1881)

Australia (New South Wales)

India (West Bengal)

India ( )

India (Bihar)

India (Bihar)

(rec.)

Australia (New South Wales)

UP-LT

UPRani

UP

LG

LGRani

LG

LGRani

LGBara

P

India (West Bengal)

LGRani

(rec.)

India (Bihar)

India (Bihar)

LGBara

LGBara

(rec.)

India (West Bengal)

LGRani

India (Madhya Pradesh)

LGRani

(rec.)

India (West Bengal)

LGRani

Australia ()

$\mathrm{P}$

(scheme from Rigby, 1972)

(from Brongniart, 1830)

South Africa (Transvaal)

(scheme)

Madagascar (Sakoa Basin)

(scheme)

South Africa (Transvaal)

India (Bihar)

India (West Bengal)

Australia (New South Wales)

India (West Bengal)

India (Assam)

Australia (New South Wales)

$\mathrm{P}$

(rec.) 
1979 Srivastava, fig.5; pl.2, 7

1980 Rigby et al., figs.7-11

G. browniana Brongniart 1830 var. indica Bunbury 1861

1976 Kovács-Endrödy, pl.6, A, B

Remark: pl.6, A is a photograph of the specimen figured on pl.67, 2 in Brongniart (1830)

G. churiensis Srivastava 1977

1977 Srivastava (b), fig. $9 ;$ pl.2, 8,9

1979 Chandra et Surange, figs.9E, 22C, 48J, 54H; pl.1, 10; pl.17, 14

1979 Chandra et Surange, pl.26, 5

G. clarkei Feistmantel 1878

1978 Banerjee (a), fig.22; pi.9, 22

1979 Banerjee, pl.50, 10, pl.54, 8-10

Remark: organic connection with Senotheca murulidihensis 1980 Rigby et al., figs.20, 22-24 (cuti), 25

G. communis Feistmantel 1876

1976 Banerji et al., fig.2C; pl.1, 9

1976 Hsü, pl.1, 1B, 5B; pl.3, 15; pl.4, 18-23

1976 Kovács-Endrödy, fig.10D

1976 Kovács-Endrödy, figs.7, 10A-C, 11; pl.1, B

1977 Appert, pls.22-24; 25, 1, 3-5

1977 Bose et al., pl.1, 13; pl.2, 22

1977 Srivastava (a), pl.2, 12

1977 Srivastava (b), pl.1, 7

1978 Banerjee (a), fig.23; pl.2, 23

1978 Hsü, pl.1, 1-4

1978 Rigby (b), figs.25, 26

1979 Banerjee et al., pl.37, 4, 5

1979 Chandra et Surange, figs.1B, 4A, B, 10C, 44E, 45E,

53D, J; pl.1, 2, 3; pl.13, 7; pl.15, 1, 6

1979 Chandra et Surange, figs.9C, 44G

1979 Chandra et Surange, figs.1C, D, 53 F

1979 Chandra et Surange, pl.26, 1

1979 Kovács-Endrödy, fig.3.4; pl.5, B

1979 Srivastava, fig. $1 ;$ pl.1, 1, 2

1980 Rigby et al., figs. $26,27-28$ (cuti), 29,30

G. conspicua Feistmantel 1881

1976 Lele (b), fig.5

1977 Srivastava (b), fig.5; pl.1, 5

1978 Banerjee (a), fig.4; pl.2, 4

1978 White, fig. 58

Remark: organic connection with Scutum sahnii

1979 Chandra et Surange, figs.27B, E, 49G, 50H; pl.5, 5;

pl.7, $1 ;$ pl.21, 6

1979 Chandra et Surange, pl.41, 6

1979 Srivastava, pl.2, 11

1980 Retallack, fig.21.5J

Remark: organic connection with fructifications

G. contracta Pant et Gupta 1971

1977 Chandra et Surange (c), figs.1, 7, 15B

1977 Chandra et Surange (c), figs.3, 6, 8; pl.1, 3, 4; pl.2, 5;

pl.3, 14; pl.5, 24, 25

Remark: descr./ills. include cuticles. Organic connection with Venustostrobus diademus

G. cordata Dana 1849

1980 Rigby et al., figs.31-33

G. cordiformis Pant et Singh 1971

1979 Chandra et Surange, figs.36A, B, 37C, 52B; pl.11, 5; pl.21, 9

1979 Chandra et Surange, pl.35, 1
India (Bihar)

UPRani

Australia (New South Wales)

$\mathbf{P}$

(from Brongniart, 1830)

India (Bihar)

LGBara

LGBara

(rec.)

Australia (New South Wales)

LP

India (West Bengal)

UPRani

Australia (New South Wales)

$P$

India (Madhya Pradesh)

China (Xizang)

(from Feistmantel, 1876)

South Africa (Transvaal)

Madagascar (Sakoa Basin)

India (Madhya Pradesch)

India (Bihar)

India (Bihar)

India (West Bengal)

China (Xizang)

Australia (Queensland)

India (Assam)

India (Bihar)

India (Bihar)

India (Madhya Pradesh)

(rec.)

South Africa (Transvaal)

India (Bihar)

Australia (New South Wales)

(scheme)

India (Bihar)

India (West Bengal)

Australia (New South Wales)

LGBara

UPRani

$P$

India (West Bengal)

LGRani

(rec.)

India (Bihar)

UPRani

Australia (New South Wales)

L-UP

(rec.)

India (West Bengal)

UPRani

Australia (New South Wales)

India (West Bengal)

LGRani
UP-LT?

P

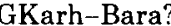

LGKarh

LGBara

UP

$\mathrm{U} ? \mathrm{P}$

LGKarh

LGBara

$P$

UPRani

$\mathrm{P}$ 
G. cyclopteroides (Feistmantel 1876) Kovács-Endrödy 1977

1977 Kovács-Endrödy (b), pl.8, 1, 2

1977 Kovács-Endrödy (b), pl.8, 3

1977 Kovács-Endrödy (b), pl.9, 1

G. damudica Feistmantel 1879

1976 Kovács-Endrödy, figs.9, 18, 19; pl.4, B-D

1977 Appert, pl.30, 13

1977 Kovács-Endrödy (b), pl.14, 4; pl.15, 1, 2

1977 Srivastava (a), fig. $4 ;$ pl.2, 10, 11

1978 Banerjee (a), fig.5; pl.3, 5

1979 Chandra et Surange, fig.34A; pl.4, 2; pl.20, 1

1979 Chandra et Surange, pl.36, 1

G. decipiens Feistmantel 1879

1978 Banerjee (a), fig.20; pl.9, 20

1979 Chandra et Surange, figs.21F, 46Q; pl.1, 5, 6; pl.15, 4

1979 Chandra et Surange, pl.26, 4

1979 Srivastava, fig. $7 ;$ pl.3, 13

G. divergens Feistmantel 1881

1976 Kovács-Endrödy, figs.27, 28; pl.5, A-C

1978 Banerjee (a), fig.6; pl.4, 6

1979 Chandra et Surange, figs.36C, D, 38d, 51C

1979 Chandra et Surange, figs.37B, 51A; pl.4, 3; pl.20, 2

1979 Chandra et Surange, pl.41, 2

1979 Srivastava, fig. $3 ;$ pl. 2,8

G. elegans Feistmantel 1879

1978 Banerjee (a), fig. $23 ;$ pl.9, 23

G. elongata Dana 1849

1980 Rigby et al., figs. $34 \cdots 35$

G. emarginata Maheshwari et Prakash 1965

1979 Chandra et Surange, figs.2C, 18E; pl.1, 11, 12; pl.15, 7, 12

1979 Chandra et Surange, pl.41, 4

G. euryneura Maheshwari 1965

1979 Chandra et Surange, figs.29D, 50D; pl.9, 2, 3; pl.19, 5

1979 Chandra et Surange, pl.39, 1

G. feistmantelii Rigby 1964

1978 Banerjee (a), fig. 7 ; pl.4, 7

1979 Chandra et Surange, figs.20A, B, 44D, 48B, pl.2, 3; pl.5, 3;

pl.10, 3 ; pl.16, 10; pl.19, 1

1979 Chandra et Surange, pl.38, 2

G. fibrosa Pant 1958

1977 Pant, fig.1B C

G. formosa Feistmantel 1881

1978 Banerjee (a), fig. 8 ; pl.4, 8

1979 Chandra et Surange, fig.28B, C; pl.4, $5 ;$ pl.21, 4

1979 Chandra et Surange, pl.44, 6

1980 Rigby et al., figs. $36-37$

G. fuchsii Plumstead 1962

1977 Srivastava (b), fig.4; pl.3, 14

G. fusa Kulkarni 1971

1979 Srivastava, fig. $8 ;$ pl.2, 9

G. ghusikensis Pant et Gupta 1971

1977 Chandra et Surange (e), fig.10; pl.4, 19, 20

1977 Chandra et Surange (e), fig.15A

Remark: descr./ills. include cuticles. Organic connection with Jambadostrobus pretiosus

G. gigas Pant et Singh 1971

1979 Chandra et Surange, figs.39C, 40d, 53C; pl.12, 1;

pl.16, 6

1979 Chandra et Surange, pl.25, 1

\begin{tabular}{|c|c|}
\hline Australia (Victoria) & $\mathbf{P}$ \\
\hline South Africa (Transvaal) & $\mathbf{P}$ \\
\hline South Africa (Transvaal) & $\mathrm{P}$ \\
\hline Madagascar (Sakoa Basin) & LGKarh-Bara? \\
\hline South Africa (Transvaal) & $\mathrm{P}$ \\
\hline India (Bihar) & LGKarh \\
\hline India (West Bengal) & LG \\
\hline $\begin{array}{l}\text { India (West Bengal) } \\
\text { (rec.) }\end{array}$ & LGBarM \\
\hline India (Bihar) & LRKarh \\
\hline $\begin{array}{l}\text { India (Bihar) } \\
\text { (rec.) }\end{array}$ & LGKarh \\
\hline India (Bihar) & UPRani \\
\hline South Africa (Transvaal) & $\mathrm{P}$ \\
\hline India (West Bengal) & UPRani \\
\hline India (Bihar) & LGRani \\
\hline $\begin{array}{l}\text { India (West Bengal) } \\
\text { (rec.) }\end{array}$ & LGRani \\
\hline India (Bihar) & UPRani \\
\hline Australia (New South Wales) & LP \\
\hline Australia (New South Wales) & $\mathrm{P}$ \\
\hline $\begin{array}{l}\text { India (Bihar) } \\
\text { (rec.) }\end{array}$ & LGBara \\
\hline $\begin{array}{l}\text { India (West Bengal) } \\
\text { (rec.) }\end{array}$ & LGRani \\
\hline India (Madhya Pradesh) & UPRani \\
\hline $\begin{array}{l}\text { India (Madhya Pradesh) } \\
\text { (rec.) }\end{array}$ & LGRani \\
\hline (from Pant, 1958) & \\
\hline India (West Bengal) & UPRani \\
\hline $\begin{array}{l}\text { India (West Bengal) } \\
\text { (rec.) }\end{array}$ & LGRani \\
\hline Australia (New South Wales) & $\mathrm{P}$ \\
\hline India (Bihar) & LGBara \\
\hline India (Bihar) & UPRani \\
\hline $\begin{array}{l}\text { India (West Bengal) } \\
\text { (rec.) }\end{array}$ & UPRani \\
\hline $\begin{array}{l}\text { India (West Bengal) } \\
\text { (rec.) }\end{array}$ & LGRani \\
\hline
\end{tabular}

South Africa (Transvaal)

Madagascar (Sakoa Basin

South Africa (Transvaal)

ngal)

India (Bihar)

India (Bihar

South Africa (Transvaal)

India (West Bengal)

UPRani

LGRani

India (West Bengal)

(rec.)

Australia (New South Wales)

Australia (New South Wales)

India (Bihar)

LGBara

India (West Bengal)

LGRani

$$
\text { Ind }
$$


G. giridihensis Pant et Gupta 1971

1979 Chandra et Surange, figs.10D, 45C

1979 Chandra et Surange, pl.47, 4

G. gondwanensis Pant et Gupta 1971

1979 Chandra et Surange, figs.29E, 41A, B, 50P, 54G; pl.13,

3,$6 ; \mathrm{pl} .22,7 ; \mathrm{pl} .23,4,10$

1979 Chandra et Surange, pl.44, 1, 3

G. guizhouensis $\mathrm{Gu}$ et Zhi 1974

1978 Zhang, pl.161, 5

G. indica Schimper 1869

1976 Hsü, pl.4, 24

1976 Kovács-Endrödy, figs.2, 4, 6, 21; pl.1, C

1976 Lele (b), fig. 5

1977 Appert, pl.25, 1, 2; pl.26; pl.27; pl.28, 1-4, 7-8(?); pl.29, 1-3

1977 Bose et al., fig.3B; pl.1, 14; pl.2, 15

1977 Correa da Silva et Arrondo, pl.1, 1

1977 Srivastava (b), fig.1; pl.1, 1, 2

1977 Zimina, pl.4, 7 (cf.)

1978 Banerjee (a), figs.9, 10; pl.5, 9; pl.6, 10

1978 Cazzulo-Klepzig, fig.4C, D; pl.3, 1

Remark: in text fig.4; pl.9, 2

1978 Correa da Silva, pl.4, 5; pl.5, 1

1978 Hsü, pl.2, 1

1978 Rigby (b), figs.25, 26

1978 Sarkar, fig.6

1978 Sarkar, fig.7

1978 Sarkar, figs. $8-10$

1979 Chandra et Surange, figs.1A, 5F, 6D, 9B, 45I, 54A

1979 Chandra et Surange, figs.2A ,7A, 45N; pl.5, 1; pl.10, 4;

pl.15, 11

1979 Chandra et Surange, figs.2B, 15C, 45J, L; pl.3, 4;

pl.10, 2; pl.16, 5; pl.18, 12

1979 Chandra et Surange, figs.2D, 53B

1979 Chandra et Surange, figs.3A, 5C, 10B, 45M

1979 Chandra et Surange, figs.6A, B, 47C, 54P

1979 Chandra et Surange, fig.17C

1979 Chandra et Surange, pl.28, 1; pl.29, 1, 2

1980 Cazzulo-Klepzig et al. (a), pl.3, 7, 15

1980 Cazzulo-Klepzig et al. (b), pl.1, 11; pl.2, 12

1980 Rigby et al., figs.38-41

G. intermedia (Feistmantel 1880) Maheshwari 1965

1978 Banerjee (a), fig.11; pl.5, 11

1979 Chandra et Surange, figs.27D, 50Q

1979 Chandra et Surange, figs.28A, 500

1979 Chandra et Surange, figs.28E, G, 29B, 50J, K, N;

pl. 6,$10 ; \mathrm{pl} .18,10$

1979 Chandra et Surange, pl.27, 2; pl.43, 1

G. intermittens Feistmantel 1881

1977 Srivastava (b), fig.8; pl.1, 3 (all cf.)

1978 Banerjee (a), fig.12; pl.7, 12

1979 Chandra et Surange, figs.11E, F, 44L; pl.3, 5; pl.17, 9

1979 Chandra et Surange, pl.41, 5

1979 Kovács-Endrödy, fig.3.3

1980 Rigby et al., figs. 42,43

G. jonesii Walkom 1922

1976 Gould, fig.3G

G. karanpurensis Kulkarni 1971

1979 Chandra et Surange, figs.33d, 41C, 52A; pl.2, 5; pl.22, 1, 2

1979 Chandra et Surange, pl.30, 1
India (Bihar) LGKarh

(rec.)

India (West Bengal) LGRani

(rec.)

China (Guizhou) UP

China (Xizang) UP

South Africa (Transvaal) P

(scheme)

Madagascar (Sakoa Basin) LGKarh-Bara?

India (Madhya Pradesh) UP-LT

Brazil (Rio Grande do Sul) LPSakm-Arti

India (Bihar)

USSR (Primorskiy Kray)

India (West Bengal)

LGBara

UP

UPRani

Brazil (Santa Catarina)

UPKaza-Tata

Brazil (Rio Grande do Sul)

China (Xizang)

LPSakm

UP

Australia (Queensland) U?P

India (Uttar Pradesh) LG

Australia (New South Wales) LG

Antarctica LG

India (Bihar) LGBara

India (Uttar Pradesh) LGKamt

India (West Bengal) LGRani

India (Bihar) LGRani

India (Maharashtra) LGKamt

India (Madhya Pradesh) $P$

India (Madhya Pradesh) LGKarh?

(rec.)

Brazil (Rio Grande do Sul) LPSakm

Brazil (Rio Grande do Sul) LPSakm

Australia (New South Wales)

India (West Bengal) UPRani

India (Bihar) LGRani

India (Bihar) LGBara

India (West Bengal) LGRani

(rec.)

India (Bihar)

LGBara

India (West Bengal)

LPBara

LGBara

(rec.)

South Africa (Transvaal)

$\mathbf{P}$

Australia (New South Wales)

$\mathbf{P}$

(scheme)

India (Bihar)

LGBara

(rec.) 
G. karharbariensis Chandra et Surange 1979

1979 Chandra et Surange, figs.26A, 49N, pl.47, 3

G. kashmirensis (Seward 1905) Kovács-Endrödy 1979

1979 Kovács-Endrödy, pl.3. B

G. lanceolatus Pant et Singh 1971

1979 Chandra et Surange, figs.5A, 36F, 38A, 40C, 47A.

52D, F; pl.7, 2; pl.19. 2

1979 Chandra et Surange, pl.40, 2

G. leptoneura Bunbury 1861

1977 Bose et al., fig. $6 \mathrm{~A}:$ pl.1, 12

1977 Srivastava (b), fig. $3:$ pl.2, 10

1979 Srivastava, pl.3, 14

G. linearis McCoy 1847

1978 White, figs. 3.4

1978 White, figs. 33.34

1978 White, fig. 35

1980 Retallack, fig.21.5I

1980 Rigby et al., figs. $18,44 \cdots 45$ (cf.)

G. longicaulis Feistmantel 1881

1977 Bose et al., fig.3A; pl.1, 11

1978 Banerjee (a), fig. $13 ;$ pl.7, 13

1979 Chandra et Surange, figs.21A, C, 46U, V; pl.1, 4; pl.15, f.13

G. maccoyi Kovács-Endrödy 1977

1977 Kovács-Endrödy (b), fig.5

1977 Kovács-Endrödy (b), pl.11, 1 2A; pl.12: pl.13, 1

1977 Kovács-Endrödy (b), pl.11, 3, 4; pl.13, 2

1979 Kovács-Endrödy. fig. 2, 2; pl.2, B

G. mackayi (Tate 1867) Kovács-Endrödy 1977

Basionym: Rubidgea mackayi Tate 1867 in

Q.J. Geol. Soc. London, 23: 141

G. maculata Pant et Singh 1974

1977 Pant, fig. 1D

1979 Chandra et Surange, figs.32A, C. 49L. M: pl.9,

6. 7 : pl.19, 3,8

1979 Chandra et Surange, figs.43D, 54B; pl.13, 2; pl.22, 8

1979 Chandra et Surange, pl.27, 3

G. major Pant et Singh 1971

1977 Kovács-Endrödy (b), figs.3, 8

1977 Kovács-Endrödy (b), pl.3, 1; pl.4, 3

1977 Kovács-Endrödy (b), pl.3, 2

Remark: in text $G$. major (Feistmante] 1879)

Kovács-Fndrödy 1977, a later homonym. New name required.

1977 Pant, fig. $1 \mathrm{~A}$

1979 Chandra et Surange, fig.42A

1979 Chandra et Surange, pl.45, 2

G. mohudaensis Chandra et Surange 1979

1979 Chandra et Surange, figs.39B, 51G; pl.11, 2; pl.18, 14

1979 Chandra et Surange, pl.46. 2

G. nakkarea Chandra et Surange 1979

1979 Chandra et Surange, figs.11D, 22H, 24A, 25I, 48M, N

1979 Chandra et Surange, figs.22G, $46 \mathrm{I}$

1979 Chandra et Surange, pl.47, 2

G. nautivalii Pant et Singh 1971

1979 Chandra et Surange, figs.6C, 16A C, 466, 47G, O): pl.8,

4: pl.18, $1 ;$ pl.23, 8,14

1979 Chandra et Surange, pl.46. 1

G. nidpurensis Srivastava 1971

1979 Srivastava, fig. $2 ;$ pl.2, 6 (all of.)

$\begin{array}{ll}\text { India (Bihar) } & \text { LGKarh } \\ \text { South Africa (Transvaal) } & \\ & \\ \text { India (West Bengal) } & \text { LGRani } \\ \text { (rec.) } & \\ \text { India (Madhya Pradesh) } & \text { UP..LT } \\ \text { India (Bihar) } & \text { LGBara } \\ \text { India (Bihar) } & \text { UPRani } \\ & \\ \text { Australia (New South Wales) } & \text { UP } \\ \text { Australia (New South Wales) } & \text { P } \\ \text { (rec) } & \\ \text { Australia (New South Wales) } & \text { L UP } \\ \text { Australia (New South Wales) } & \text { P } \\ & \\ \text { India (Madhya Pradesh) } & \text { UP..LT } \\ \text { India (Bihar) } & \text { LPKarh } \\ \text { India (Bihar) } & \text { LGKarh } \\ & \\ \text { (scheme) } & \text { P } \\ \text { Australia (Victoria) } & \text { Pouth Africa (Transvaal) } \\ \text { South Africa (Transvaal) } & \text { P }\end{array}$

(from Pant et Singh, 1974)

India (West Bengal) LGRani

India (Orissa) IJGRani

(rec)

(scheme)

(from Feistmantel. 1879)

South Africa (Transvaal)

$\mathrm{P}$

(from Pant et Singh, 1971)

India (West Bengal)

LGRani

(rec)

India (West Bengal)

LGRani

(rec.)

India (Bihar)

LGBara

India (West Bengal)

(rec)

LGBara

India (West Bengal)

LGRani

(rec)

India (Bihar)
UPRani 
G. nimishea Chandra et Surange 1979

1979 Chandra et Surange, figs.17A, B, 43C, 47K, 48C, 53A; pl. $2,2,7 ; \mathrm{pl} .16,7,8$

1979 Chandra et Surange, figs.35A, D, 52H; pl.4, 1; pl.6, 5;

pl.18, $4 ; \mathrm{pl} .19,10$

1979 Chandra et Surange, figs.35B, $51 \mathrm{~F}$

1979 Chandra et Surange, pl.45, 11

G. obliqua (McCoy 1875) Kovács-Endrödy 1977

1977 Kovács-Endrödy (b), fig.6

1977 Kovács-Endrödy (b), pl.6, 1; pl.7, 20

1977 Kovács-Endrödy (b), pl.6, 2

1977 Kovács-Endrödy (b), pl.6, 3

1979 Kovács-Endrödy, pl.8, B

G. obovata (Carruthers 1869) Kovács-Endrödy 1977

1977 Kovács-Endrödy (b), figs.2, 7

1977 Kovács-Endrödy (b), pl.1, 1, 2

1977 Kovács-Endrödy (b), pl.2, 1

1977 Kovács-Endrödy (b), pl.2, 2, 3

G. oldhamii Pant et Singh 1974

1979 Chandra et Surange, figs.36E, 52G; pl.8, 3; pl.19, 9

1979 Chandra et Surange, pl.37, 1

G. orbicularis Feistmantel 1881

1978 Banerjee (a), figs.14, 15; pl.7, 14, 15

1979 Chandra et Surange, figs.7B, 18D, 49C; pl.3,

1,$2 ; \mathrm{pl} .21,7,8$

1979 Chandra et Surange, pl.26, 2

G. orientalis Zimina 1967

1977 Zimina, fig.10; pl.2, 1-5

G. pandurata Pant et Gupta 1971

1977 Srivastava (b), fig. 7 ; pl.1, 6

1979 Chandra et Surange, figs.22A, 46T; pl.8, 2; pl.17, 6

1979 Chandra et Surange, pl.43, 6

G. pantii Chandra et Surange 1979

1979 Chandra et Surange, figs.3B, 18B, 21D, 23I, 30D, 47N,

48D, G, 54C, F; pl.13, 4; pl.14, 1; pl.22, 6, 10, 14

1979 Chandra et Surange, figs.18C, $53 \mathrm{~K}$

1979 Chandra et Surange, figs. $30 \mathrm{H}, 47 \mathrm{M}$

1979 Chandra et Surange, pl.31, 1

G. parallela Feistmantel 1878

1978 Banerjee (a), fig.24; pl.10, 24

1979 Banerjee et al., pl.36, 1

G. pseudocommunis Kovács-Endrödy 1976

1976 Kovács-Endrödy, figs.12, 22-26; pl.2, A-D

Remark: a later homonym of G.pseudocommunis

Pant et Gupta 1968. New name:

G. andreanskyi Kovács-Endrödy 1977

G. radiata Pant et Singh 1971

1979 Chandra et Surange, figs.32B, 49K; pl.9, 4; pl.22, 5

G. raniganjensis Chandra et Surange 1979

1979 Chandra et Surange, figs.4E, 8A,B, 44K; pl.3, 3; pl.10,

$1 ;$ pl.16, 2, 3

1979 Chandra et Surange, figs.8C, $44 \mathrm{~F}$

1979 Chandra et Surange, fig.9A

1979 Chandra et Surange, figs.9D, 44H

1979 Chandra et Surange, fig.40B

1979 Chandra et Surange, pl.3, 8; pl.16, 1

1979 Chandra et Surange, pl.4, 4; pl.5, $2 ;$ pl.15, 2

1979 Chandra et Surange, pl.24, 1

$\begin{array}{ll}\text { India (Bihar) } & \text { LGBara } \\ \begin{array}{l}\text { India (West Bengal) } \\ \text { India (-) } \\ \text { (rec.) }\end{array} & \begin{array}{l}\text { LGBara } \\ \text { (scheme) }\end{array} \\ \begin{array}{l}\text { Australia (Victoria) } \\ \text { (from Feistmantel, 1881) }\end{array} & \\ \begin{array}{l}\text { South Africa (Transvaal) } \\ \text { (from McCoy) }\end{array} & \text { P } \\ \begin{array}{l}\text { (scheme) } \\ \text { Brazil (Rio Grande do Sul) } \\ \text { South Africa (Transvaal) } \\ \text { (from Feistmantel, 1879) }\end{array} & \text { P } \\ \begin{array}{l}\text { India (West Bengal) } \\ \text { (rec.) }\end{array} & \text { P } \\ \begin{array}{l}\text { India (West Bengal) } \\ \text { India (Assam) }\end{array} & \text { LGRani } \\ \begin{array}{l}\text { India (West Bengal) } \\ \text { (rec.) }\end{array} & \text { Pouth Africa (Transvaal) } \\ \begin{array}{l}\text { India (Bihar) } \\ \text { (rec.) }\end{array} & \text { LG } \\ \begin{array}{l}\text { USSR (Primorskiy Kray) } \\ \text { India (Bihar) }\end{array} & \text { UPRani } \\ & \text { LGRani } \\ & \\ & \end{array}$

India (West Bengal)

LGRani

India (West Bengal)

LGRani

India (Uttar Pradesh)

LGKamt

India (Bihar)

LGBara

LGRani

LGBarM

India (Bihar)

India (Bihar)

LGRani

India (Maharashtra)

LGKamt 
G. rectinervis Dun 1897

1978 Banerjee (a), fig.25; pl.10, 25

G. recurva Pant et Singh 1974

1979 Chandra et Surange, figs.13A, 24B, 25, 47B, 54N; pl.6, 8; pl.17, 11

G. retifera Feistmantel 1881

1976 Banerji et al., fig. $3 \mathrm{E} ;$ pl.1, 10

1977 Bose et al., fig.4; pl.2, 21

1978 Banerjee (a), fig.16; pl.8, 16

1979 Chandra et Surange, figs.27A, 30B, 31A, C, D, E,

49D, F, H, J, 50F; pl.5, 6; pl.21, 1, 5; pl.23, 5, 13

1979 Chandra et Surange, figs.28F, $50 \mathrm{I}$

1979 Chandra et Surange, pl.26, 3

1979 Srivastava, fig. $4 ;$ pl.2, 10

G. retusa Maheshwari 1965

1979 Chandra et Surange, figs.22B, D, 48H; pl.6, 6; pl.7, 8; pl.15, 3

1979 Chandra et Surange, pl.43, 8

1980 Rigby et al., figs.46, 47

G. rewaensis Chandra et Surange 1979

1979 Chandra et Surange, fig.19E

1979 Chandra et Surange, figs.26I, 50G

1979 Chandra et Surange, figs.33C, 50C

1979 Chandra et Surange, pl.47, 6

G. rhabdotaenioides Pant et Singh 1971

1979 Chandra et Surange, figs.34B, 35C, 37A, 39A,

$51 \mathrm{E}, \mathrm{H}, 52 \mathrm{E}, 53 \mathrm{H}$; pl.9, 5; pl.13, 1 ; pl.18, 6 ; pl.20, 3

1979 Chandra et Surange, pl.33, 1

G. sahnii Srivastava 1956

1979 Chandra et Surange, figs.37d, 38C, 42d, 52C, I;

pl.11, 1, 4; pl.20, 4, 5

1979 Chandra et Surange, pl.34, 1

G. saksenae Chandra et Surange 1979

1979 Chandra et Surange, figs.11h, 26J, 54I

1979 Chandra et Surange, figs.15D, 54M

G. sastrii Pant et Singh 1974

1977 Pant, fig. 4

1979 Chandra et Surange, figs.29A, 50E; pl.9, 1; pl.19, 4

1979 Chandra et Surange, pl.39, 2

G. searsolensis Pant et Singh 1974

1979 Chandra et Surange, figs.29C, 50M; pl.23, 6, 12

1979 Chandra et Surange, pl.47, 1

G. spathulato-cordata Feistmantel 1878

1978 Banerjee (a), fig.26; pl.7, 26

G. spatulata Pant et Singh 1971

1979 Chandra et Surange, figs.14A, 25F, 45A, 46W; pl.8.

1; pl.12, 5; pl.17, 7; pl.18, 1

1979 Chandra et Surange, figs.30G, 54E, L

1979 Chandra et Surange, pl.27, 1

G. srivastavae Surange et Maheshwari 1962

1979 Chandra et Surange, figs.32D, 50A; pl.11, 3; pl.19. 6

1979 Chandra et Surange, pl.47, 7

G. stenoneura (Feistmantel 1881) Banerji et al. 1976

1976 Banerji et al., figs.3A-D, 4A-B; pl.2, 16-18

1979 Chandra et Surange, figs.11A, 44I; pl.1, 8; pl.15, 8; pl.17, 4

1979 Chandra et Surange, figs.11B, 12A, 44J; pl.1, 7; pl.17. 1

1979 Chandra et Surange, pl.41, 1

G. stricta Bunbury 1861

1978 Banerjee (a), figs.17, 18; pl.8, 17, 18
Australia (New South Wales)

UP

India (Bihar)

LGKarh

India (Madhya Pradesh)

India (Madhya Pradesh)

India (West Bengal)

India (West Bengal)

India (Bihar)

(rec.)

India (Bihar)

UP-LT?

UP--LT

UPRani

LGRani

LGBara

UPRani

India (West Bengal)

LGRani

(rec.)

Australia (New South Wales)

$\mathrm{P}$

India (Madhya Pradesh)

India (Bihar)

India (West Bengal)

(rec.)

India (West Bengal)

LGRani

(rec.)

India (West Bengal)

LGRani

(rec.)

India (Bihar)

India (Madhya Pradesh)

LGBara

LGBara

India

LG

India (West Bengal)

LGRani

(rec.)

India (West Bengal)

LGRani

(rec.)

Australia (New South Wales)

UP

India (West Bengal)

LGRani

India (Madhya Pradesh)

(rec.)

India (West Bengal)

LGRani

(rec.)

India (Madhya Pradesh)

India (Bihar)

India (West Bengal)

(rec.)

India (Maharashtra)
UP LT?

LGBara

LGBara

LGKarh?

UPRani 
1979 Banerjee et al., pl.36, 3

1979 Chandra et Surange, fig.33A

1979 Chandra et Surange, figs.33B, 38B; pl.5, 4

1979 Chandra et Surange, fig.51J

1979 Chandra et Surange, pl.32, 1

1979 Srivastava, fig.6; pl.1, 3

G. subtilis Pant et Gupta 1971

1979 Chandra et Surange, figs.15A, B, 17d, 20C, D,

47I, J, 48A; pl.3, 7; pl.8, 5, 7; pl.14, 3, pl.17, 16;

pl.21, $2 ; \mathrm{pl} .22,3,12$

1979 Chandra et Surange, pl.37, 2

G. syaldiensis Chandra et Surange 1979

1979 Chandra et Surange, figs.40A, 51B; pl.2, 1; pl.15, 5

1979 Chandra et Surange, pl.43, 4

G. taeniensis Chandra et Surange 1979

1979 Chandra et Surange, figs.19A, 27C, 47I, 49A; pl.23, 7

1979 Chandra et Surange, figs.19B, 47E; pl.23, 9

1979 Chandra et Surange, pl.43, 5

G. taenioides Feistmantel 1882

1977 Chandra et Surange (a), figs.1, 2A, B; pl.1,

$1-4 ; \mathrm{pl} .2,5,6 ; \mathrm{pl} .3,10-11$ (cuti)

1977 Chandra et Surange (a), figs.6, 7

Remark: organic connection with Plumsteadiostrobus ellipticus

1977 Chandra et Surange (e), fig.14B

Remark: organic connection with Plumsteadiostrobus ellipticus

1977 Pant, fig.9L

1978 Banerjee (a), fig.19; pl.8, 19

1979 Chandra et Surange, figs.26D, 49O; pl.4, 6; pl.18, 5

1979 Chandra et Surange, pl.43, 3

G. taeniopteroides Feistmantel 1878

1976 Kovács-Endrödy, figs.8, 14-17; pl.2, E;

pl.3, A-C; pl.4, A

1978 Banerjee (a), fig.27; pl.10, 27

1979 Chandra et Surange, fig.43A (cf.)

G. talchirensis Chandra et Surange 1979

1979 Chandra et Surange, figs.4C, 5D, E, 44A, B; pl.1, 1

G. tenuifolia Pant et Gupta 1968

1979 Chandra et Surange, figs.12C, F, H, 13B, C, 14E,

21B, 23A, J-L, 25A, D, 44M, 45B, 46A-D, R, 54J, Q; pl.6,

1,$2 ; \mathrm{pl} .7,6 ; \mathrm{pl} .14,4,7 ; \mathrm{pl} .15,10 ; \mathrm{pl} .17,10,12 ; \mathrm{pl} .22,9 ; \mathrm{pl} .42,1,6$

G. tenuinervis Pant et Gupta 1971

1976 Chandra et Surange, figs.1-4; pls.1-8

1976 Chandra et Surange, fig.7

Remark: descr./ills. include cuticles.

Organic connection with

Dictyopteridium feistmantelii

1977 Chandra et Surange (e), fig.14A

Remark: organic connection with Dictyopteridium feistmantelii

1979 Chandra et Surange, figs.13E, 19F, 30A, 42B, 47L, 51D,

54D; pl.6, 3; pl.7, 3; pl.14, 6; pl.18, 2; pl.22, 4, 13; pl.23,

1,15

1979 Chandra et Surange, pl.46, 3

G. tortuosa Zeiller 1902

1979 Chandra et Surange, figs.19H, 49E; pl.2, ô; pl.18, 9

1979 Chandra et Surange, figs.42c, 43B, 54K; pl.14, 5; pl.22, 11

1979 Chandra et Surange, pl.43, 2

1979 Kovács-Endrödy, fig.3.2; pl.4, A; pl.8, A; pl.10, A, B
India (Assam)

LG

India (Bihar)

India (Uttar Pradesh)

LGKamt

LGKamt

LGRani

(rec.)

India (Bihar) UPRani

India (West Bengal) LGRani

(rec.)

India (Bihar) LGBara

(rec.)

India (Bihar) LGBara

India (Bihar) LGKarh

(rec.)

India (West Bengal) LGRani

(rec.)

(rec.)

(from Pant et Singh, 1974)

India (Madhya Pradesh) LPKarh

India (Bihar) LGKarh

(rec.)

South Africa (Transvaal) P

Australia (New South Wales) UP

India (Uttar Pradesh) LG

India (Bihar) TGTalc

India (West Bengal) LGRani

India (West Bengal) UPRani

(rec.)

(rec.)

India (West Bengal) LGRani

(rec.)

India (Bihar)

LGBara

India (West Bengal) LGRani

(rec.)

South Africa (Transvaal) P 
+ G. tunguscana (Nejburg) Zimina

1977 Zimina, pl.4, 8 (cf.)

G. varia Pant et Gupta 1968

USSR (Primorskiy Kray)

UP

1979 Chandra et Surange, figs.24C, E, N, 25h, J,

$48 \mathrm{~K}, \mathrm{~L} ;$ pl.7, 5 ; pl.17, f.5

1979 Chandra et Surange, pl.25, 2, 11

G. vulgaris Pant et Gupta 1968

1979 Chandra et Surange, figs.13F, 460; pl.6, $9 ;$ pl.15, 9

(rec.)

1979 Chandra et Surange, pl.42, 5

G. wilkinsonii Feistmantel 1878

1978 Banerjee (a), fig.28; pl.10, 28

G. zeilleri Pant et Gupta 1968

1979 Chandra et Surange, figs.13D, 45K; pl.6, 4; pl.17, 13

1979 Chandra et Surange, pl.42, 3

$\begin{array}{ll}\begin{array}{l}\text { India (Bihar) } \\ \text { (rec.) }\end{array} & \text { LGRani } \\ \begin{array}{l}\text { India (Bihar) } \\ \text { (rec.) }\end{array} & \text { LGRani } \\ \text { Australia (New South Wales) } & \text { UP } \\ \text { India (Bihar) } & \text { LGKarh }\end{array}$

\section{Glossotheca Surange et Maheshwari 1970}

G. sp.

1976 Schopf, fig.9F

1977 Pant, fig.8E

1977 Schweitzer, fig.8A

G. immanis Chandra et Surange 1977

1977 Chandra et Surange (c), fig.1A; pl.1, 2, 5

1978 Surange et Chandra, pl.1, 1

G. orissiana Surange et Chandra 1974

1977 Chandra et Surange (c), fig.1B

1978 Surange et Chandra, pl.3, 1

1980 Daber (a), fig.1D

G. utkalensis Surange et Maheshwari 1970

1976 Schopf, fig.9E

1977 Chandra et Surange (c), fig.1C

1978 Surange et Chandra, pl.1, 2

\section{Glottophyllum Zalessky 1912}

G. sp.

1976 Durante, pl.55, 3, 4; pl.57, 4-5 (?)

G. cuneatum Zalessky 1912

1976 Durante, pl.55, 5-7; pl.56, 6

G. karpovii Radchenko 1960

1976 Durante, pl.55, 8; pl.56, 7

1978 Miller, fig.2C

G. keuperiana (Goeppert 1848) Schimper 1870-1872

1978 Miller, fig. $2 \mathrm{E}$

\section{Gnetopsis Renault et Zeiller 1884}

G. sp.

1979 Taylor et Millay, fig.3D

\section{Gomphostrobus Marion 1890}

G. bifidus (Geinitz 1873) Potonié 1891

1977 Tenchov (b), pl.4, 2

Bulgaria (Northwest)

LP

\section{Gondwanidium Gothan 1927}

G. sp.

1976 Anderson et McLachlan, pl.1, 3 (cf.) (rec. from Florin, 1951)

(from Renault et Zeiller, 1884)
Mongolia

Mongolia

Mongolia

(rec. from Florin, 1951)
LGRani

LG

$\mathrm{p}$

LG

UP

UP

UP

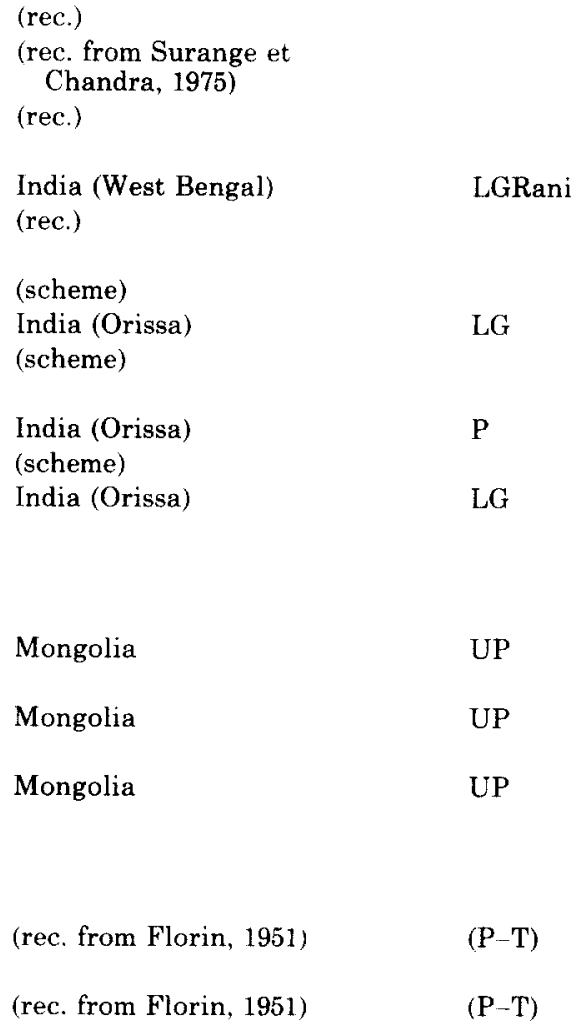


1976 Anderson et McLachlan, pl.2, 13 (cf.)

1976 Balasundaram et al., pl.22, 3

1976 Bohlin, figs.223 (cf.), 713 (cf.), 716; pl.14, 1 (cf.)

1979 Kovács-Endrödy, pl.1, A

\section{Gothanopteris Koidzumi 1936}

G. bosschana (Jongmans et Gothan 1935) Koidzumi 1936 1976 Asama, pl.30, 5

Indonesia (Sumatra)

LP

\section{Grammatopteris Renault 1891}

G. baldauffii (Beck 1920) Hirmer 1927

1976 Barthel et al., pl.2, 3

GDR (Karl-Marx-Stadt)

LPRotl

\section{Guizhoua Zhao 1980}

G. gregalis Zhao 1980

1980 Zhao et al., pl.23, 1, 2, 3-4 (spores in situ)

China (Guizhou/Yunnan)

UP

\section{Hagiophyton Corsin 1948}

H. sp.

$1980 \mathrm{X}$, number " 6 " in fig.

(rec. from Corsin, 1948)

\section{Henanophyllum Xi et Feng 1977}

H. palamifolium Xi et Feng 1977

1977 Feng et al., pl.248, 6, 7

China (Henan)

UP

\section{Heterangium Corda 1845}

$H$. sp.

1980 Tian et Zhang, pl.25, 6(?)

China (Guizhou)

UP

\section{Hirsutum Plumstead 1958}

$H$. sp.

1977 Pant, fig.9I-K

(from Plumstead, 1958)

\section{Holcospermum Nathorst 1914}

$H$. sp.

1978 Gillespie et al., pl.37, 8; pl.47, 9, 10

H. sulcatum (Presl 1838) Seward 1917

1976 Barthel et al., pl.39, 4; pl.40, 10

\section{Huihuipuia Bohlin 1976}

H. reticulata Bohlin 1976

1976 Bohlin, fig.222; pl.13, 9, 10

Indocarpus Surange et Chandra 1974

I. $\mathrm{sp}$.

1977 Pant, fig.6F

USA (West Virginia)

UC-LP?

GDR (Karl-Marx-Stadt)

LPRotl

China (Kansu)

UC-LP

Iniopteris (Zalessky 1918) Zalessky 1934

I. sp.

1976 Durante, pl.52, 5

Mongolia

UP

1980 Durante, fig.4

China (Xizang)

UP

I. sibirica Zalessky 1934

1977 Huang, fig.7; pl.9, 1-3; pl.10, 2-3

China (Heilongjiang)

UP 


\section{Intia Nejburg 1958}

I. falciformis Nejburg 1956 ex Nejburg 1960

1978 Fefilova (b), fig.6; pl.5, 1, 2

1978 Fefilova (b), pl.5, 3 (aff.)

I. variabilis Nejburg 1956

1978 Fefilova (b), figs.3, 4; pls.2, 3

I. vermicularis Nejburg 1958

1978 Fefilova (b), fig.2; pl.1

1979 Lacey, fig.1(3)

I. vicaria Fefilova 1978

1978 Fefilova (b), fig. 5 , pl.4

$\begin{array}{ll}\text { USSR (Komi ASSR) } & \begin{array}{l}\text { LPUfim } \\ \text { UPTata }\end{array} \\ \text { USSR (Komi ASSR) } & \text { LPUfim } \\ \text { USSR (Komi ASSR) } & \text { LPUfim } \\ \begin{array}{l}\text { USSR (Komi ASSR) } \\ \text { (from Nejburg, 1960) }\end{array} & \\ \text { USSR (Komi ASSR) } & \text { LPUfim }\end{array}$

Isodictyopteridium Rigby 1972

I. costatum Holmes 1974

1976 Schopf, fig.9A

I. walkomii Rigby 1972

1978 Rigby (a), figs.17, 18

\section{Jaganovia Radchenko et Verbitskaya 1980}

J. ziminkensis Verbitskaya 1980

1980 Verbitskaya (a), fig.4; pl.4, 7, 8; pl.5, 1

\section{Jambadostrobus Chandra et Surange 1977}

$J$. pretiosus Chandra et Surange 1977

1977 Chandra et Surange (e), figs.2, 4, 5, 6; pl.1;

pl.2; pl.3, 13, 15-18; pl.5, 24

1977 Chandra et Surange, figs.7, 15B

Remark: descr./ilis. include cuticles and seeds Platycardia bengalensis

Organic connection with Glossopteris ghusikensis

1978 Surange et Chandra, pl.4, 2

1979 Chandra et Surange, pl.14, 4, 7; pl.22, 9

Remark: organic connection with Glossopteris tenuifolia

\section{Junjagia Nejburg 1960}

J. glottophylla Nejburg 1960

1978 Fefilova (b), fig.18; pl.18

\section{Kamaraspermum Kern et Andrews 1946}

$K . \mathrm{sp}$.

1976 Bohlin, fig.567

Remark: in text Camarospermum

\section{Kashmiropteris Kapoor 1979}

K. meyenii Kapoor 1979

1979 Kapoor, pl.169, 1, 2

India (Jammu and Kashmir)

$\mathrm{P}$

Kawizophyllum Kapoor 1979

K. dunpathriensis Kapoor 1979

1979 Kapoor, pl.170, 2

Kendostrobus Surange et Chandra 1974

$K$. sp.

1977 Pant, fig.8A
Australia (New South Wales)

UP

Australia (Queensland)

UP

USSR (Kemerovskaya oblast -

Kuzbass --)

LP

India (West Bengal)

UPRani (rec.)

(rec.)

India (West Bengal)

LGRan

USSR (Komi ASSR)

LPUfim (from Kern et Andrews, 1946)

India (Jammu and Kashmir)

$\mathbf{P}$ 
K. cylindricus Surange et Chandra 1974 1978 Surange et Chandra, pl.3, 4

\section{Knorria Sternberg 1825}

$K . \mathrm{sp}$.

1977 Feng et al., fig.101

1977 Feng et al., pl.233, 6

1979 He et al., pl.50, 5, 6

(scheme)
China (Henan)
China (Guizhou)

(C-P)

UP

UP

(scheme)

China (-) UP

China (-)

(scheme)

$\mathbf{P}$

1979 Asama, fig.3(8)

1980 Asama, fig.5

\section{Koretrophyllites Radchenko 1955}

$K$. sp.

1980 Andreis et al., pl.1, 1, 3, 4; pl.2, 2, 4

1980 Cazzulo-Klepzig et al. (b), pl.2, 8

K. crassinervis Broutin 1974

1977 De la Peña et al., pl.2, 3

$K$. gracilis Verbitskaya 1980

1980 Verbitskaya (b), pl.6, 2, 3

K. latifolius Verbitskaya et Radchenko 1980 1980 Verbitskaya (b), pl.6, 4, 5

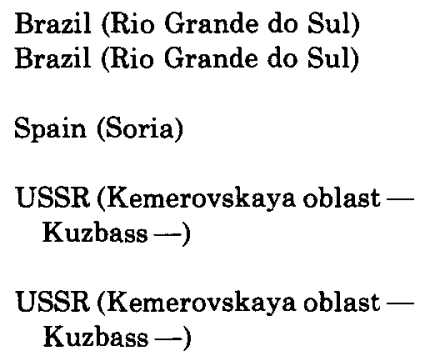

LP

LPSakm

LPAutu

LP

LP

\section{Kosjunia Fefilova 1973}

K. polyedra Fefilova 1973

1978 Fefilova (b), fig.13a, v; pl.12, 2, 3

USSR (Komi ASSR)

USSR (Komi ASSR)

LPUfim

1978 Fefilova (b), fig.13b, g; pl.12, 1

K. retusa Fefilova 1973

1978 Fefilova (b), fig.14; pl.13

USSR (Komi ASSR)

UPKaza

L.PUfim

\section{Lanceolatus Plumstead 1952}

L. sp.

1977 Pant, fig.9H

L. lerouxides Plumstead 1952

1976 Schopf, fig.1("')')

(from Plumstead, 1958)

(scheme)

\section{+ Laxostrobus}

L. sp.

1976 Durante, pl.71, 10-12

1978 Durante et Biterman, pl.23, 7

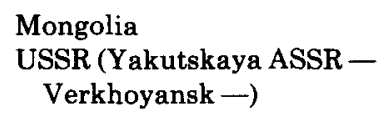

UP

UP

\section{Lebachia Florin 1938}

L. sp.

1976 Barthel (b), pl.3, 8

1976 Barthel et al., pl.41, 2 (cuti)

1978 Bariand et Pajaud, figs. p.18, 19

Remark: occurrence according to text Carboniferous 1979 Major, fig.212

1979 Yuasa, fig.5.6

$1980 \mathrm{X}$, number " 9 " in fig.

GDR (Karl-Marx-Stadt)

GDR (Karl-Marx-Stadt)

France

LPRotl

LPRotl

$\mathrm{P}$

(scheme)

(C-P)

(rec. from Florin, 1938) 
L. frondosa (Renault 1885) Florin 1939-1945

1976 Barthel et al., pl.42, 1

1977 Tenchov (b), pl.4, 1, 6

GDR (Dresde

LPRotl

Bulgaria (Northwest)

LP

1976 Barthel (b), pl.4. 2

1976 Barthel et al., pl.41, 3

1980 Bold et al., fig. 24 - 31B

L. hypnoides (Brongniart 1849) Florin 1939-1945

1978 Remy et Remy (c), pl.8, 15

L. parvifolia Florin 1939 1945

1977 Tenchov (b), pl.3, 8; pl.4, 8

L. piniformis (Sternberg 1825) Florin 1938

1976 Harris, fig.2G, H

1976 Mundlos et al., fig. p.89

1976 Rothe, fig.23

1976 Teixeira et Pais, fig. 39

1977 De la Peña et al., pl.2, 4, 5

1977 Krumbiegel et Walther, fig.2.101.1

1977 Krumbiegel et Walther, fig.2.101.3

1977 Remy et Remy, fig.43B

1977 Tenchov (b), pl.3, 3

1977 Vogellehner (a), fig. p.57

1977 Vogellehner (b), fig.2

1978 Doubinger et al., pl.2, 1-3

1978 Grauvogel-Stamm, fig.44C

1978 Miller, fig.1A

1978 Strasburger, fig.792A-C

1979 Asama, fig.12E, F

1979 Delevoryas, fig. 10

1979 Richter, fig. 170

1979 Richter, fig. 174

1980 Kämpfe, fig.7.13.D

L. piniformis (Sternberg 1825) Florin 1938

var. solmsii Florin 1938

1980 Bold et al., fig. 24 31A

L. speciosa Florin 1939-1945

1976 Daber et Helms, fig. p.75

GDR (Leipzig)

LPRot]

GDR (Leipzig)

LPRotl

(scheme from Florin)

Italy (Brescia)

LPSaxo

Bulgaria (Northwest)

LP

(scheme)

(C-P)

GFR (Saarland)

LPRotl

GDR (Erfurt)

LPRotl

(from Emberger, 1968)

LPAutu

Spain (Soria)

LPRotl

(rec. from Gothan et Weyland)

GDR (Suhl)

Bulgaria (Northwest)

LPAutu

LP

(from Mägdefrau, 1968)

Spain (Gerona)

$\mathrm{P}$

(scheme from Schweitzer, 1963)

LPAutu

(rec. from Florin, 1951)

(scheme from Florin)

(scheme from Florin, 1944)

(from Florin, 1944)

France (Hérault)

France (Aveyron)

LPAutu

(scheme from Zimmermann, 1959)

(rec. from Florin)

GFR (Saarland)

UC $\mathbf{L P}$

\section{Lelstotheca Maheshwari 1972}

Remark: substitute name for Stellotheca Surange et Prakash 1962 non Stellotheca Danzé-Corsin 1956. See Farr et al. (1979 p.951)

L. robusta (Feistmantel 1880) Maheshwari 1972 1980 Retallack, fig.21.5D

Australia (New South Wales)

L--UP

\section{Lepeophyllum Zalessky 1933}

L. sp.

1976 Durante, pl.71, 9

1978 Remy et Remy (c), pl.7, 13-15(?)

1980 Goman'kov et Meien, fig.1(4)

L. actaeonelloides (Geinitz 1871) Zalessky 1933

1976 Durante, pl.71, 2, 3

L. belovoense Gorelova 1962

1976 Durante, pl.71, 48

L. gemmatum Zalessky 1933

1980 Huang, pl.259, 14 15; pl.260, 5-6

$L$. liaoningense Huang 1980

1980 Huang, pl.244, 8-9; pl.259, 13, 13A

Mongolia

Italy (Brescia)

(scheme)

Mongolia

Mongolia

China (Liaoning)

China (Liaoning)
UP

LPSaxo

UP

UP

UP

UP 
L. ovatum $\mathrm{Hu} 1980$

$1980 \mathrm{Hu}, \mathrm{pl} .1,5,5 \mathrm{~A}$

China (Xinjiang)

UP

L. trigonum Nejburg 1965

China (Heilongjiang)

UP

1977 Huang, pl.25, 4-5

USSR (Primorskiy Kray)

LP

\section{Lepidodendron Sternberg 1820}

L. sp.

1976 Bohlin, fig.495

1976 Bohlin, fig.701

1976 Bohlin, fig.730

1976 Bohlin, pl.30, 5-6, 9-11

1976 Teixeira et Pais, fig. 17

1976 Teixeira et Pais, fig. 18

1977 Feng et al., fig. 100

1978 Gillespie et al., fig.6

1978 Ratkevich et La Fon, fig. p.49

1978 Yuasa, fig.5.3

1980 Babin, fig.8.6A,B, 8.7

1980 Huang, pl.233, 5-7

1980 Tian et Zhang, fig. 1

1980 Tian et Zhang, pl.2, 3; pl.3, 1, 4, 4A

L. aculeatum Sternberg 1820

1978 Gillespie et al., pl.11, 1

1978 Gillespie et al., pl.11, 2

L. acutangulum (Halle 1927) Stockmans et Mathieu 1957

1977 Feng et al., pl.232, 7, 8

1978 Zhang, pl.150, 4

1980 Tian et Zhang, pl.2, 2, 4-7; pl.3, 2, 2A

1980 Zhao et al., pl.2, 1, 2; pl.5, 7

L. carinum Lee 1963

1976 Huang, pl.204, 2, 2A

L. cervicisum Sze 1954

1977 Feng et al., pl.233, 3

L. gaudryi Renault 1888-1890

1976 Bohlin, pl.30, 7, 8

L. henanense Feng 1977

1977 Feng et al., pl.232, 4, 5

L. incertum Sze et Lee 1946

1977 Feng et al., pl.234, 6

L. latiligulatum Bohlin 1976

1976 Bohlin, figs.304-312, 317-334, 338, 339,

$431,433,435,436,438,441,629$; pl.18, 3-9; pl.19,

$1-6 ;$ pl.31, 7, 8

L. lepidophloides Yao 1980

1980 Zhao et al., pl.3, 1, 1A, 2, 6

L. oculus-felis (Abbado 1900) Zeiller 1901

1976 Bohlin, pl.30, 3-4

1977 Feng et al., pl.232, 6

1978 Zhang, pl.150, 6, 7

1980 Tian et Zhang, pl.2, 1

1980 Zhao et al., pl.2, 4-6

L. pingdingshanense $\mathrm{Xi} 1977$

1977 Feng et al., pl.233, 8

L. polygonale Gu et Zhi 1974

1977 Feng et al., pl.233, 1

L. posthumii Jongmans et Gothan 1935

1977 De la Peña et al., pl.2, 6; pl.3, 1, 2

\begin{tabular}{|c|c|}
\hline (from Gothan) & $(\mathrm{C}-\mathrm{P})$ \\
\hline (scheme) & $(\mathrm{C}-\mathrm{P})$ \\
\hline China (Chinghai) & $\mathrm{UC}-\mathrm{LP}$ \\
\hline China $(-)$ & UC-LP \\
\hline (rec.) & $(\mathrm{C}-\mathrm{P})$ \\
\hline (scheme) & $(\mathrm{C}-\mathrm{P})$ \\
\hline (rec.) & $(\mathrm{C}-\mathrm{P})$ \\
\hline (rec.) & (UC-LP?) \\
\hline USA (New Mexico) & LP \\
\hline (scheme from Hirmer, 1927) & $(\mathrm{C}-\mathrm{P})$ \\
\hline (scheme) & $(\mathrm{D}-\mathrm{P})$ \\
\hline $\begin{array}{l}\text { China (Liaoning) } \\
\text { (rec.) }\end{array}$ & LP \\
\hline China (Guizhou) & UP \\
\hline $\begin{array}{l}\text { USA (West Virginia) } \\
\text { (scheme) }\end{array}$ & $\begin{array}{l}\text { UC-LP? } \\
\text { (UC-LP?) }\end{array}$ \\
\hline China (Guangdong) & UP \\
\hline China (Guizhou) & UP \\
\hline China (Guizhou) & UP \\
\hline China (Guizhou/Yunnan) & $\mathrm{UP}$ \\
\hline China (Inner Mongolia) & $\mathrm{LP}$ \\
\hline China (Henan) & UP \\
\hline (from Halle, 1927) & \\
\hline China (Henan) & LP \\
\hline China (Henan) & LP \\
\hline China (Chinghai) & UC-LP \\
\hline China (Guizhou/Yunnan) & UP \\
\hline (from Halle, 1927) & \\
\hline China (Guangdong) & UP \\
\hline China (Guizhou) & UP \\
\hline China (Guizhou) & UP \\
\hline China (Guizhou/Yunnan) & UP \\
\hline China (Henan) & LP \\
\hline China (Henan) & UP \\
\hline Spain (Soria) & LPAutu \\
\hline
\end{tabular}


L. tachingshanense Lee 1963

1976 Huang, pl.206, 1-2A

L. worthenii Lesquereux 1866

1978 Gillespie et al., pl.11, 7; pl.12, 2 (all cf.)

L. xingningense Feng 1977

1977 Feng et al., pl.233, 9, 10

L. xuanweiense Yao 1980

1980 Zhao et al., pl.2, 3

$\begin{array}{ll}\text { China (Inner Mongolia) } & \text { LP } \\ \text { USA (West Virginia) } & \text { UC-LP? } \\ \text { China (Guangdong) } & \text { UP } \\ \text { China (Guizhou/Yunnan) } & \text { UP }\end{array}$

Lepidodendropsis Lutz 1933

L. sp.

1978 Bohlin, figs.1-15(??)

Canada (-)

C? - P?

\section{Lepidophyllum Brongniart 1828}

Remark: non Lepidophyllum Cassini 1816. See Farr et al. (1979, p.959)

L. sp.

1977 Huang, pl.23, 4-6

China (Heilongjiang)

UP

\section{Lepidopteris Schimper 1869}

L. ottonis (Goeppert 1836) Schimper 1869

1979 Delevoryas, fig.3A

(from Delevoryas, 1962)

\section{Lepidostrobus Brongniart 1828}

L. sp.

1980 Babin, fig.8.9

L. acutisquamus Yao 1980

1980 Zhao, pl.2, 7, 8

(scheme)

(D-P)

China (Guizhou/Yunnan)

UP

\section{Lescuropteris Schimper 1869}

L. moorii (Lesquereux 1858) Schimper 1869

1977 Remy et Remy, fig.129A

1977 Remy et Remy, fig.129B

1978 Gillespie et al., pl.49, 1, 2

\section{Lesleya Lesquereux 1880}

L. sp.

1977 Appert, pl.6 (cf.)

L. eckhardtii (Germar ex Kurtze 1839) Remy et Remy 1977

1977 Remy et Remy, fig.69

1978 Remy et Remy (c), pl.8, 10-11 (cf.)

L. gimmii Remy et Remy 1978

1978 Remy et Remy (a), fig.1; pl.16, 1-3

\section{Lidgettonia Thomas 1958}

L. sp.

1976 Schopf, fig.8B, C; pl.3, 2-5; pl.4, 6

1976 Schopf, fig.8D

1977 Pant, fig.8I, J

1978 White, figs.52, 53

1978 White, fig.54

Remark: organic connection with cupules

L. africana Thomas 1958

1976 Schopf, fig.8A; pl.3, 1

1977 Pant, fig.9F

1979 Van Dijk et al., pl.46, 20-22
USA (Pennsylvania)

(scheme)

USA (West Virginia)

LPAutu

UC-LP?

Madagascar (Sakoa Basin)

LGTalc - Karh?

(scheme)

Italy (Brescia) LPSaxo

GFR (Bayern)

LPAutu

\author{
South Africa (Natal) \\ (rec.) \\ (rec. from Surange et \\ Chandra, 1975)
}

$\mathrm{P}$

Australia (New South Wales) UP

(rec.)

South Africa (Natal)

$\mathrm{P}$

(from Thomas, 1958)

South Africa (Natal) 
L. mucronata Surange et Chandra 1974 1977 Chandra et Surange (c), fig.2A

(scheme)

1978 Srivastava, pl.2, 1

India (Orissa)

UPRani

1980 Daber (a), fig.1A

(scheme)

\section{Lilpopia Conert et Schaarschmidt 1970}

L. crockensis (Remy et Remy 1961) Conert et Schaarschmidt 1970 1977 Remy et Remy, fig.248A-F, G (spore)

\section{Linophyllum Zhao 1980}

L. xuanweiense Zhao 1980

1980 Zhao et al., pl.22, 5, 5A

China (Guizhou/Yunnan)

UP

\section{Linopteris Presl 1838}

L. sp.

1976 Bohlin, figs.242, 269; pl.12, 14; pl.14, 5 (all ?)

1980 Huang, pl.240, 6

L. semianastomosa Tenchov 1977

1977 Tenchov (a), pl.2, 2, 3

China (Kansu)

China (Liaoning)

Bulgaria (Northwest)
UC-LP

LP

LP

\section{Lobatannularia Kawasaki 1927}

L. sp.

1977 Feng et al., fig.105

1978 Gillespie et al., pl.28, 5

1979 Asama, fig.5 (9-12)

L. cathaysiana Yao 1980

1980 Zhao et al., pl.3, 3; pl.5, f.1-6

L. ensifolia (Halle 1927) Halle 1928

1977 Feng et al., pl.238, 13

1978 Chen et al., pl.151, 2

L. fusiformis Zhang 1978

1978 Zhang, pl.152, 2, 3

L. heianensis (Kodaira 1924) Kawasaki 1927

1976 Asama, fig.1(21)

1977 Feng et al., pl.238, 10, 11

1977 Feng et al., pl.238, 12

1977 Huang, pl.40, 2-3

1978 Chen et al., pl.150, 2; pl.152, 1

Remark: in text $L$. peianensis

1978 Zhang, pl.152, 6

1979 Asama, fig.3(21)

1980 Asama, fig.5

1980 Huang, pl.225, 8-9 (cf.)

L. huapingensis Feng 1977

1977 Feng et al., pl.237, 7

L. lingulata (Halle 1927) Halle 1928

1976 Asama, fig.1(20)

1977 Feng et al., pl.238, 1, 2

1978 Chen et al., pl.150, 3

1979 Asama, fig.3(20)

1980 Asama, fig.5

1980 Huang, pl.219, 1-5

1980 Tian et Zhang, pl.5, 3; pl.6, 1, 4 (all cf.)

1980 Zhao et al., pl.5, 8

L. longifolia Feng 1977

1977 Feng et al., pl.237, 5, 6

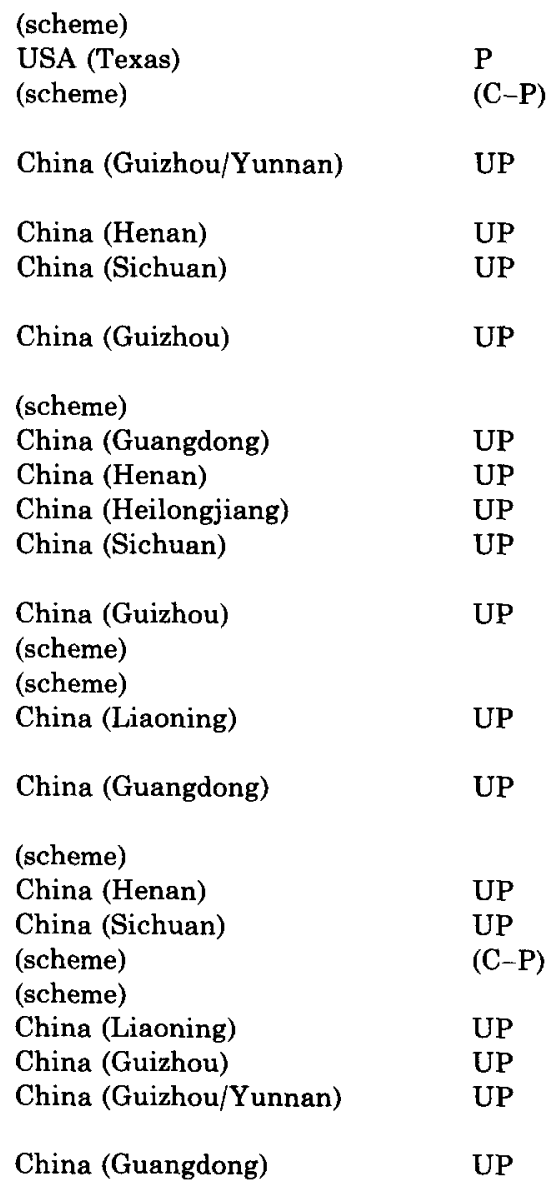


L. multifolia Konno et Asama 1950

1977 Feng et al., pl.237, 14

1977 Huang, pl.40, 46

1978 Chen et al., pl.151, 1

1978 Zhang, pl.152, 1

1980 Tian et Zhang. pl.5, 11 (cf.)

1980 Zhao et al., pl.6. 7 9

L. sinensis (Halle 1927) Halle 1928

1976 Asama, fig.1(19)

1977 Feng et al., pl.238, 8

1979 Asama, fig.3(19)

1980 Asama, fig.o

China (Guangdong)

China (Heilongjiang) UP

China (Sichuan) UP

China (Guizhou) UP

China (Guizhou) UP

China (Guizhou/Yunnan) UP

(scheme)

China (Henan) UP

\section{Lopadiangium Zhao 1980}

L. acmodontum Zhao 1980

1980 Zhao et al., pl.23. 914

China (Guizhou/Yunnan)

UP

Lophotheca Zhao 1980

L. panxianensis Zhao 1980

1980 Zhao et al., pl.23. 5, 6, 7.8 (spores in situ)

China (Guizhou/Yunnan)

UP

\section{Lycopodiopsis Renault 1890}

L. sp.

1980 Lemoigne et Brown (b), figs.1, 2

1980 Lemoigne et Brown, pl.1, 1- 7; pl.2, 1 4;

pl.5, 1 7: pl.7, 1

1980 Lemoigne et Brown, pl.6, 1 4; pl.7, 2, 3, 5

(scheme)

(scheme)

Remark: descr./ills, include cuticles

L. costilatus Lemoigne et Brown 1980

1980 Lemoigne et Brown (a), pl.2, 610

1980 Lemoigne et Brown (b), pl.8, 2- 3

L. derbyi Renault 1890

1979 Arrondo et Petriella, fig.1C; pl.1, 1-6

1980 Lemoigne et Brown (a), pl.1, 1 5

1980 Lemoigne et Brown (b), pl.3, 1, 2;

1980 Lemoigne et Brown, pl.3, 3, 4; pl.4, 13 ;

pl.7. 4, 6; pl.8, 1, 6

Remark: descr./1lls. include cuticles and spores

L. millanii Arrondo et Petriella 1979

1979 Arrondo et Petriella, fig.1D; pl.2, 1-5

L. pedroanus (Carruthers 1869) Edwards 1952

1979 Arrondo et Petriella, fig.1A, B; pl.3. 15

(scheme)

South Africa

South Africa (Transvaal)

LG

LG

Namibia (Kaoko Veld) U?P

South Africa LG

Argentina (Chubut; La Rioja) C? P

Namibia (Kaoko Veld) U?P

South Africa LG

South Africa (Transvaal) LG

Argentina (Chubut; La Rioja) MC--LP

Argentina (Chubut; La Rioja; San Juan)

MC-LP

\section{Macrostachya Schimper 1869}

$M$. sp.

1976 Bohlin, figs.13-16. 29; pl.1, 11; pl.2, 11:

pl.27. 1 (all cf.)

China (Kansu)

UC-LP

M. carinata (Germar 1851) Zeiller 1878

1976 Barthel et al, fig.9, pl.29, 15

Remark: organic connection with

GDR (Karl-Marx-Stadt)

LPRotl Calamites crassicaulis

\section{Marattiopsis Schimper 1870}

M. sp.

1980 El-Khayal et al., figs.2D, E

Saudi Arabia ('Unayzah)

UC $\mathrm{LP}$

M. orientalis Burago 1977

1977 Burago, pl.23, 1 4; pl.24, 1 4; pl.25, 2 .

4. $5:$ pl.26, 2,3

USSR (Primorskiy Kray)

UP 


\section{Mariopteris Zeiller 1879}

M. crassipinnulata Bohlin 1976

1976 Bohlin, figs.182, 186, 188, 191, 194; pl.11, 3,

$11,16,17 ; \mathrm{pl} .13,6,7 ; \mathrm{pl} .27,11 ; \mathrm{pl} .32,9,18$

China (Kansu)

UC-LP

M. yuemenensis Bohlin 1976

1976 Bohlin, fig.449; pl.12, 13 (all ?)

China (Kansu)

UC-LP

\section{Masculostrobus Seward 1911}

$M$. sp.

1977 Jung, pl.18, 2, 5

\section{Medullosa Cotta 1832}

$M$. sp.

1976 Grzimek, fig.p.51

1976 Grzimek, fig. 8.22

(scheme)

(UC-P)

1976 Stewart, fig. 2

(scheme)

1976 Stewart, figs.7-8, 10

(diagram)

M. elegans Cotta 1832

1885 Quenstedt, pl.96, 4

GDR (Karl-Marx-Stadt) LPRotl

M. leuckartii Göppert et Stenzel 1881

1977 Krumbiegel et Walther, pl.51, 86

M. stellata Cotta 1832

1885 Quenstedt, pl.96, 5

1976 Barthel (b), pl.4, 10

1976 Barthel et al., pl.30, 5, 6

1976 Daber et Helms, fig. p.72

1977 Krumbiegel et Walther, pl.18, 31

1977 Remy et Remy, fig.33B (cf.)

1978 Daber et Helms, fig. p.174

GDR (Karl-Marx-Stadt)

LPRotl

GDR (Karl-Marx-Stadt)

LPRotl

GDR (Karl-Marx-Stadt)

LPRotl

GDR (Karl-Marx-Stadt)

LPRotl

GDR (Karl-Marx-Stadt)

LP

GDR (Karl-Marx-Stadt)

LPRot1

GDR (Karl-Marx-Stadt)

LPAutu

GDR (Karl-Marx-Stadt)

LPRotl

\section{Mesocalamites Hirmer 1927}

+ M. gigas (Brongniart 1828)

1977 Remy et Remy, fig.215

GFR (Saarland)

LPAutu

\section{Millaya Mapes et Schabilion 1979}

M. tularosana Mapes et Schabilion 1979

1978 Ratkevich et La Fon, fig. p.49

USA (New Mexico)

LP

\section{Mixoneura Weiss 1870}

M. sp.

1976 Bohlin, figs.221, 224-226 (cf.); pl.11,

$$
1 \text { (cf.), } 2,4
$$

China (Kansu)

UC-LP

\section{Mooia Lacey, Van Dijk et Gordon-Gray 1975}

M. lidgettonioides Lacey, Van Dijk et Gordon-Gray 1975

1977 Pant, fig.6D, E

1978 Surange et Chandra, pl.2, 3

(from Lacey et al., 1975)

South Africa (Natal)

UP

\section{Moyliostrobus Miller et Brown 1973}

M. texanum Miller et Brown 1973

1978 Miller, fig.1B

(rec. from Miller et Brown, 1973) 
M. brasiliense Mussa 1978

1978 Mussa (c), pl.7. 39-45; pl.8, 46-48

Brazil (São Paulo)

M-UP

M. vittii Mussa 1978

1978 Mussa (c), fig.3B

1978 Mussa (c), fig.5; pl.4, 18-23; pl.5, 24-29;

pl.6, 30-37

(rec.)

Brazil São Paulo)

M-UP

\section{Myeloxylon Brongniart 1849}

$M$. sp.

1977 Remy et Remy, fig.33C, D

1980 Rumpel, figs.1, 2

M. elegans (Cotta 1832) Brongniart 1849

1976 Barthel et al., pl.40, 8

GDR (Karl-Marx-Stadt)

GFR (Bayern)

GDR (Karl-Marx-Stadt)

GDR (Karl-Marx-Stadt)

GDR (Karl-Marx-Stadt)

China (Kansu)

(rec. from Barthel)

GDR (Karl-Marx-Stadt)

(scheme)

(scheme)

GDR (Dresden)

GDR (Gera)

GDR (Halle)

GFR (Hessen)

India (Bihar)

India (Bihar)

India (Orissa)

India (Madhya Pradesh)

Australia (New South Wales)

Australia (New South Wales)

India (Maharashtra)

N. polymorpha (Feistmantel 1876) Maithy 1974

1979 Chitnis et Vagyani, figs.1, 2; pl.1, 1

\section{Nephropsis Zalessky 1912}

N. sp.

1977 Correa da Silva et Arrondo, pl.1, 2 (cf.)

1977 Zimina, pl.19, 10; pl.20, 8 10

1980 Cazzulo-Klepzig et al. (a), pl.3, 2 (cf.)

N. acuminata Radchenko 1962

1977 Zimina, pl.20, 7 (aff.)

N. asiatica Zimina 1969

1977 Zimina, pl.18, 16; pl.19, 12-14

1978 Zimina, pl.2, 13
Brazil (Rio Grande do Sul)

Brazil (Rio Grande do Sul)

USSR (Primorskiy Kray)

USSR (Primorskiy Kray)

USSR (Primorskiy Kray)
USSR (Primorskiy Kray)
LPAutu

UC-P

LPRotl

LPRotl

LPRotl

UC-LP

LPRotl

(C--P)

(C-P)

C-P

LPRotl

UPKupf UPKupf

LPBara

LGKarh

UPKamt

UP-LT

L-UP

$\mathrm{P}$

UP-LT

LPSakm-Arti

LP

LPSakm

LP

LP

LP 
N. cochlearis Nejburg 1965

1980 Huang, pl.248, 17

China (Heilongjiang) LP

N. elegans Huang 1980

1980 Huang, pl.248, 15

N. integerrima (Schmalhausen 1876) Zalessky 1912

1977 Zimina, pl.20, 5-6, 11-12 (aff.)

N. lampadiformis (Gorelova 1962) Zimina 1969

1977 Zimina, pl.18, 15

N. pospeloviensis Zimina 1969

1977 Zimina, pl.15, 11; pl.18, 11, 12

1978 Zimina, pl.2, 6

N. pseudosimilis Burago 1979

1979 Burago, pl.9, 4; pl.10, 2

N. rhomboidea Nejburg 1934

1980 Huang, pl.248, 16

China (Heilongjiang) LP

USSR (Primorskiy Kray) LP

USSR (Primorskiy Kray) LP

USSR (Primorskiy Kray) LP

USSR (Primorskiy Kray) LP

USSR (Primorskiy Kray) LP

China (Heilongjiang) LP

\section{Nesowalesia Pant 1977}

N. edwardsii Pant 1977

1977 Pant, fig.11A

Australia (New South Wales)

LG

\section{Neurodontopteris Potonié 1893}

$N$. sp.

1976 Bohlin, fig.220; pl.11, 8

China (Kansu)

UC-LP

\section{Neurophyllites Zhang 1980}

$N$. pecopteroides Zhang 1980

1980 Zhao et al., pl.14, 1-2B

China (Guizhou/Yunnan)

UP

\section{Neuropteridium (Schimper 1869) Weiss 1869}

$N$ coreanicum Koiwai 1927

1977 Feng et al., pl.243, 10

1978 Chen et al., pl.152, 4-5

1978 Zhang, pl.158, 4

N. guizhouense Zhang 1980

1980 Zhao et al., pl.14, 3, 3A

Remark: Same species as $N$. guizhouensis Zhang 1978?

N. guizhouensis Zhang 1978

1978 Zhang, pl.158, 1

N. nervosum Halle 1927

1977 Feng et al., pl.243, 3-4(?)

N. polymorphum Halle 1927

1977 Feng et al., pl.243, 1

N. tieyingensis Huang 1980

1980 Huang, fig.32; pl.224, 1-3; pl.225, 1-2

$\begin{array}{lr}\text { China (Guangdong) } & \text { UP } \\ \text { China (Sichuan) } & \text { UP } \\ \text { China (Guizhou) } & \text { UP } \\ \text { China (Guizhou/Yunnan) } & \text { UP } \\ & \\ \text { China (Guizhou) } & \text { UP } \\ \text { China (Guangxi) } & \text { UP } \\ \text { China (Guangdong) } & \text { UP } \\ \text { China (Liaoning) } & \text { UP }\end{array}$

\section{Neuropteris Sternberg 1825}

$N$. sp.

1976 Bohlin, figs.208-211, 214, 216-219, 220 bis(?), $227,516,552,556,720,722 ;$ pl.10,6-11; pl.11, 7,

14 (cf.); pl.12, 10-11

1976 Bohlin, figs.228 (aff.), 390, 399-422, 442, 457, 637-640; pl.22,

9-11; pl.23, 7-8; pl.24, 10-13; pl.29, 9 (cf.); pl.30, 16

1977 Minato, pl.4-1, 3; 4-2, 1, 2

1979 Major, fig.206

China (Kansu)

Chile (Valdivia)

(scheme)

LP
LP
LP
LP
LP
LP
LP

LP
$\mathrm{LP}$
$\mathrm{LP}$
$\mathrm{LP}$


1979 Yuasa, fig.s.1011

(scheme from Stewart et
Delevoryas, 1956)

Remark: in text Nouropteris N. auriculata Bronsiniart 1830

1977 Tenchov (a). pl.3, 1

N. cordata Brongniart 1830

1976 Barthel (b), pl.2, 9. 10

1976 Barthel et al.. fig.11: pl.32, $16,7 \cdot 10$ (cuti):

pl.34, 1 10.11 (cuti)

N. dagangensis Huang 1980

1980 Huang. fig. $30:$ pl.221. 13

N. daheshenensis Huang 1980

1980 Huang, fig. 31

1980 Huang, pl.250. $13 ;$ pl.251, 14

1980 Huang, pl.251, 5, 5A

N. fimbriata Lesquereux 1860

1978 Gillespie et al., pl.43, 3, 4

N. neuropteroides (Goeppert 1836) Zeiller 1907

Remark: in text $N$. neuropteroides (Goeppert 1836) Barthel

1976 Barthel (b), pl.4. 9

1976 Barthel et al., fig. 12 : pl.35, 14 (cuti), 512

1979 Doubinger et Alvarez-Ramis, pl.2. 2, $2 \mathrm{~A}$

N. ovata Hoffmann 1826

1978 Gillespie et al., pl.41, 1

1978 Gillespie et al., pl.43, 1, 2

N. planchardii Zeiller 1888

1976 Barthel et al.. pl.32, 12 (cuti), 3, 4 (cuti); pl.39, 10 (cuti)

1977 Tenchov (a), pl.3, 4 (cf.)

N. pseudovata Gothan et Sze 1933

1976 Bohlin, figs.207, 212, 213, 215: pl.10, 4 5:

pl.11, ; 6: pl.12. + (all $\mathrm{cf}$.)

N. scheuchzeri Hoffmann 18231829

1978 Gillespie et al., pl.2. 1; pl.42, 1 8; pl.46. 8

\section{Niazonaria Radchenko 1933}

N. sp.

1976 Durante, pl.71, 1(?)

\section{Nilsonia Brongniart 1825}

Remark: commonly spelled Nilssonia. See Farr et al. (1979, p.1177)

N. sp.

1980 Tian et Zhang, pl.14, 3

N. hongshanensis Huang 1977

1977 Huang, fig. 17 ; pl.14, 1.4

N. huabeiensis Gu et 7hi 1974

1980 Huang, pl.239. $\times 10 \mathrm{~A}$

\section{Noeggerathia Sternberg 1821}

N. zamitoides Sterzel 1909

1976 Barthel et al., pl.30, 11, 12

\section{Noeggerathiopsis Feistmantel 1879}

N.sp.

1976 Balasundaram et al., pl.22, 12

1976 Lele (b), fig. 2

1977 Bernardes de Oliveira et Da Silva Pontes. figs.4 5(?); pl.3, 2 3(?)
Mongolia

UP

GDR (Karl-Marx-Stadt)

LPRotl

Bulgaria (Northwest) LP

GDR (Karl-Marx-Stadt) IPRot

(iDR (Karl-Marx-Stadt) LPRot

(hina (Jilin) LP

(scheme)

(hina (Jilin) LP

China (Heilongjiang) LP

USA (West Virginia) UC LP?

GDR (Karl-Marx-Stadt) LPRot]

GDR (Karl-Marx-Stadt) LPRotl

France (Vosges) LPAutu

(scheme)

USA (West Virginial

GDR (Karl-Marx-Stadt) LPRotl

Bulgaria (Northwest) LP

China (Kansu)

UC LP

USA (West Virginia)

UC IP?

(China (Guizhou)

UP

China (Heilongjiang)

UP

China (Liaoning)

L.P

(scheme)

(scheme)

Brazil (Santa Catarına)
LPArti 
N. aequalis (Göppert 1845) Zalessky 1918

1980 Huang, pl.249, 7-8 (cf.)

$\begin{array}{ll}\text { China (Heilongjiang) } & \text { LP } \\ \begin{array}{ll}\text { China (Heilongjiang) } \\ \text { China (Heilongjiang) }\end{array} & \text { UP } \\ \text { China (Heilongjiang) } & \text { UP } \\ \text { China (Heilongjiang) } & \text { LP } \\ & \text { LP } \\ \text { China (Heilongjiang) } & \\ \text { India (Bihar) } & \text { LP } \\ \text { China (Heilongjiang) } & \text { LGKarh } \\ \text { China (Heilongjiang) } & \text { LP } \\ \text { China (Heilongjiang) } & \text { LP }\end{array}$

N. angustifolia Nejburg 1948

1977 Huang, pl.26, 4 (cf.)

1980 Huang, pl.249, 10-11 (cf.)

N. batschatensis Radchenko 1955

1977 Huang, pl.43, 4-5

1980 Huang, pl.249, 9 (cf.)

Remark: in text $N$. batshatensis

N. brevifolia Gorelova 1960

1980 Huang, pl.248, 9

N. densinervis Maithy 1965

1977 Srivastava (a), fig.6; pl.1, 3

$N$. derzavinii (Nejburg 1936) Nejburg 1948

1977 Huang, pl.42, 1-3; pl.43, 3

1980 Huang, pl.249, 1-2

N. ensiformis Zalessky 1937

1980 Huang, pl.249, 3-5

$+N$. hislopii (Bunbury 1881) Feistmantel 1879

1976 Gould, fig.4F, G

1977 Appert, pl.33; pl.34, 1-3

1977 Bernardes de Oliveira et Da Silva Pontes,

figs.2, 3; pl.1, 1-5; pl.2, 1-5; pl.3, 1, 4

1977 Correa da Silva et Arrondo, pl.2, 4

1978 Cazzulo-Klepzig, pl.3, 6

Remark: in text pl.11, 1.

$N$. insignis Radchenko 1940

1977 Huang, pl.32, 2; pl.41, 3-5

N. kryshtofovichii Radchenko 1955

1980 Huang, pl.248, 11-12 (cf.)

N. latifolia Nejburg 1948

1977 Huang, pl.8, 4-5 (cf.)

N. lianzhushanensis Huang 1980

1980 Huang, pl.248, 7, 13-14

N. mishanensis Huang 1980

1980 Huang, fig.46; pl.248, 10

N. obovata Nejburg 1965

1977 Huang, pl.33, 4

N. spathulata (Dana 1849) Maithy 1965

Remark: in text $N$. spatulata

1980 Retallack, fig. $21.5 \mathrm{~N}$

N. synensis Zalessky 1930

1980 Huang, pl.258, 4

$N$. theodori Zalessky et Chirkova 1930

1980 Huang, pl.247, 5

N. tieliensis Huang 1977

1977 Huang, pl.25, 3; pl.43, 1-2

N. xinganensis Huang 1977

1977 Huang, fig. 16 ; pl.43, 6

(scheme from Walkom, 1921)

Madagascar (Sakoa Basin)

LGKarh-Bara?

Brazil (Santa Catarina)

Brazil (Rio Grande do Sul)

LPArti

Brazil (Santa Catarina)

LPSakm-Arti

UPKaza-Tata

China (Heilongjiang) UP

China (Heilongjiang) LP

China (Heilongjiang) LP

China (Heilongjiang) UC-LP

China (Heilongjiang) P

China (Heilongjiang) LP

Australia (New South Wales) L-UP

China (Heilongjiang) UP

China (Heilongjiang) LP

China (Heilongjiang) UP

China (Heilongjiang) UP

\section{Nucicarpus Nejburg 1965}

N. piniformis Nejburg 1965

1977 Mejen, pl.7, 14, 15

USSR (Komi ASSR -- Pechora Basin -)

UP

\section{Nummulospermum Walkom 1921}

$N$. sp.

1976 Schopf, pl.5, 1-2(?)

Antarctica (Queen Alexandra Range)
$\mathrm{P}$ 
Odontopteris (Brongniart 1822) Sternberg 1825

O. aequalis Lesquereux 1866

1978 Gillespie et al., pl.48, 1, 5

USA (West Virginia)

$\mathrm{UC}-\mathrm{P}$

O. brardii (Brongniart) 1825

1978 Gillespie et al., pl.48, 2

1978 Gillespie et al., pl.48, 3, 4, 6-8

$O$. chui Lee 1963

1980 Huang, pl.241, 5, 5A

O. gimmii Remy et Remy 1958

1977 Sopeña et al., fig. 4.4

O. henanensis Xi 1977

1977 Feng et al., pl.240, 2

O. laceratifolia Halle 1927

1976 Bohlin, figs.285, 578; pl.15, 15 (all cf.,?)

O. lingulata (Göppert 1846) Schimper 1869

(scheme)

(UC-P)

USA (West Virginia)

$\mathrm{UC}-\mathrm{P}$

China (Liaoning)

LP

Spain (Guadalajara)

LPAutu

China (Henan)

LP

China (Chinghai)

UC $-\mathrm{LP}$

1976 Barthel et al., pl.30,

1976 Barthel et al., pl.38, 10

1977 Remy et Remy, fig.169A

1977 Remy et Remy, fig.169B, C

O. orbicularis Halle 1927

1977 Feng et al., pl.240, 3, 4

+ O. osmundaeformis (Schlotheim 1804) Zeiller 1879-1880

1977 Remy et Remy, fig.170A, B

O. schlotheimii Brongniart 1828

1885 Quenstedt, pl.90, 19

$O$. xinganensis Huang 1977

1977 Huang, fig.24; pl.24, 2 (all ""?)

O. yiyangensis Feng 1977

1977 Feng et al., pl.240, 7

GDR (Leipzig)

LPRotl

GDR (Karl-Marx-Stadt)

LPRotl

GFR (Rheinland-Pfalz)

LPAutu

(scheme from Gothan et Remy. 1957)

China (Henan)

LP

GDR (Gera)

LPAutu

GDR (Gera)

LPRotl

China (Heilongjiang)

UP

China (Henan)

LP

\section{Oligocarpia Göppert 1841}

$O$. sp.

1977 Remy et Remy, fig.58

(scheme)

(C-P)

\section{Osmundacaulis Miller 1967}

O. stumkei Erasmus 1977(?)

1979 Heron, fig. p. 2

South Africa (Zululand)

$$
\mathrm{P}
$$

\section{Otofolium Gu et Zhi 1974}

O. ovatum Gu et Zhi 1974

1977 Feng et al., pl.252, 4

1977 Feng et al., pl.252, 5

1978 Zhang, pl.165, 12, 13

1980 Zhao et al., pl.22, 3, 3A

O. polymorphum Gu et Zhi 1974

1977 Feng et al., pl.252, 6

China (Guangdong)
China (Henan)
China (Guizhou)
China (Guizhou/Yunnan)
China (Guangdong)

(scheme)
(rec.)
(rec. from Pant et Nautiyal)
(rec. from Surange et Chandra,
$\quad 1975$ )
India
(rec.)

UP

UP

UP

UP

UP

\section{Ottokaria Zeiller 1902}

$O$. sp.

1976 Balasundaram et al., pl.22, 10

1976 Schopf, fig.2("')

1977 Pant, fig.10E-G

1977 Pant, fig. 10H I

1977 Pant, fig.11C - F (cuti; pollen)

Remark: organic connection with Glossopteris

1977 Schweitzer, fig.8B 
1978 Banerjee (b), fig.19A-C

1978 White, figs.63, 64

O. bengalensis Zeiller 1902

1976 Schopf, fig.1("')

1976 Schopf, fig.2

1976 Schopf, fig.3F

1976 Schopf, fig.4A-B (cf.)

1977 Pant, fig.12A-C

Remark: organic connection with seeds and

Glossopteris indica

Fig.12A is Zeiller's holotype

1978 Banerjee (b), figs.1A-C, $2-9$

Remark: descr./ills. include cuticles and associated pollen grains: Virkkipollenites obscurus Lele 1964

1979 Chandra et Surange, pl.13, 7

Remark: organic connection with Glossopteris communis

O. biharensis Srivastava 1978

1978 Srivastava, fig. $3 ;$ pl.2, 9-11

O. raniganjensis Banerjee 1978

1978 Banerjee (b), figs.10A-F, 11-18

Remark: descr./ills. include cuticles and seeds. Seeds contain monosaccate pollen grains of Virkkipollenites Lele 1964

1979 Banerjee, pl.49, 1-3, 4-5 (seed), 6-8; pl.53,

$1-3,4-5$ (seed)

1979 Banerjee, pl.53, 6-7

Remark: descr./ills. includes cuticles.

According to text $O$. ranigenjensis Banerjee 1975

\section{Pachytesta Brongniart 1874}

$P$. sp.

1976 Barthel et al., pl.39, 1

\section{Palaeogoniopteris Koidzumi 1936}

P. menkarangensis (Jongmans et Gothan 1935) Koidzumi 1936 1976 Asama, pl.30, 4

\section{Palaeosmunda Gould 1970}

P. sp.

1980 Gould et Shibaoka, fig.6H

$P$. playfordii Gould 1970

1980 Bold et al., fig.19-67

\section{Palaeospiroxylon Prasad et Chandra 1980}

$P$. heterocellularis Prasad et Chandra 1980

1980 Prasad et Chandra, fig.1A-L; pl.1, 1-13

\section{Palaeostachya Weiss 1876}

P. sp.

1977 Feng et al., fig.106

1977 Remy et Remy, fig.234A

1979 Yuasa, fig.4.3

P. superba (Weiss 1884) Jongmans et Kukuk 1913

1976 Barthel et al., pl.26, 11, 12 (spore)
Indonesia (Sumatra)

LP

(rec.)

Australia (New South Wales)

UP

(scheme)

(rec.)

(from Mukherjee et al., 1966)

Argentina (Buenos Aires)

$\mathrm{P}$

India

LGKarh

India (Bihar)

LGKarh

India (Bihar)

LGKarh

India (Bihar)

LPBara

India (West Bengal)

UPRani

India (West Bengal)

UPRani

(rec.)

GDR (Leipzig)

LPRotl

(scheme)

(scheme from Gould)

India (West Bengal)

UPRani

(rec.)

(C-P)

(scheme)

(C-P)

(scheme from Baxter, 1955)

(C-P)

GDR (Karl-Marx-Stadt)

LPRotl 
P. thuringiaca (Weiss 1876) Barthel 1980 1980 Barthel (a), fig. 92

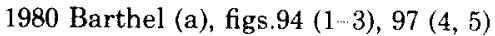

(scheme)

Remark: fig.97(4) is the specimen originally figured by

Schlotheim (1804: pl.1, 2) as Calamites interruptus.

Fig.97(5), the lectotypus of Palaeostachya thuringiaca, was originally figured by Weiss (1876: pl.2, 4) as "Stachannularia (?) thuringiaca"

\section{Palaeovittaria Feistmantel 1877}

P. sp.

1976 Balasundaram et al., pl.22, 8

1976 Lele (b), fig.2

(scheme)

(scheme)

USSR (Primorskiy Kray)
1977 Zimina, pl.3, 5, 6

UP

\section{Palaeoxylon Brongniart 1849}

Remark: non Palaeoxylon Hartig 1848. See Farr et al. (1979, p.1245)

P. sp.

1977 Marguerier, pl.1, 3

\section{Palmatophyllites Maithy 1965}

P. wardii (Zeiller 1902) Maithy 1977

1977 Maithy (c), pl.1, 1

India (Bihar)

UPKarh

\section{Paracalamites Zalessky 1932}

\section{P. sp.}

1977 Brown, figs. 1 - 3

1979 Rösler, fig.4(7)

1980 Tian et Zhang, fig. 17

P. australis Rigby 1966

1977 Correa da Silva et Arrondo, pl.2, 5 (cf.)

1978 Cazzulo-Klepzig, fig.4A, B; pl.3, 2

Remark: in text fig. $4 \mathrm{~A}, \mathrm{~B}$; pl.4, 1-2

1978 Correa da Silva, pl.5, 7

1980 Cazzulo-Klepzig et al. (a), fig.1 (cf.); pl.3, 13

1980 Cazzulo-Klepzig et al. (b), pl.2, 9

1980 Guerra-Sommer et al., pl.1, 1 (cf.)

P. crassus Gorelova 1973

1979 Burago, pl.6, 1, 2; pl.7, 1

P. decoratus (Eichwald 1860) Zalessky 1927

1979 Burago, pl.7, 5

$P$. stenocostatus $\mathrm{Gu}$ et Zhi 1974

1977 Feng et al., pl.236, 10

1978 Chen et al., pl.150, 5

1978 Zhang, pl.151, 4

1980 Tian et Zhang, pl.5, 2; pl.6, 2, 3, 5

1980 Zhao et al., pl.3, 4, 4A; pl.4, 1, 2

\section{Paracalamostachys Weiss 1884}

P. heterospora Remy et Remy 1958

1980 Barthel (a), fig.96(6, 7-8 (spores))

\section{Paracordaites}

P. sp.

1977 De la Peña et al., pl.3, 3
GDR (Suhl)

LPRotl

$\begin{array}{ll}\begin{array}{l}\text { Namibia (-) } \\ \text { (scheme) } \\ \text { China (Guizhou) }\end{array} & \text { P } \\ \begin{array}{l}\text { Brazil (Rio Grande do Sul) } \\ \text { Brazil (Santa Catarina) }\end{array} & \begin{array}{l}\text { LPSakm-Arti } \\ \text { UPKaza-Tata }\end{array} \\ \begin{array}{l}\text { Brazil (Rio Grande do Sul) } \\ \text { Brazil (Rio Grande do Sul) } \\ \text { Brazil (Rio Grande do Sul) } \\ \text { Brazil (Rio Grande do Sul) }\end{array} & \text { LPSakm } \\ & \text { LPSakm } \\ \text { USSR (Primorskiy Kray) } & \text { LPSakm } \\ & \text { LP } \\ \text { USSR (Primorskiy Kray) } & \text { LP } \\ \text { China (Guangdong) } & \text { UP } \\ \text { China (Sichuan) } & \text { UP } \\ \text { China (Guizhou) } & \text { UP } \\ \text { China (Guizhou) } & \text { UP } \\ \text { China (Guizhou/Yunnan) } & \text { UP }\end{array}$

Spain (Soria)

LPAutu 


\section{Paranocladus Florin 1940}

P. fallax Florin 1940

1978 Fittipaldi et Rösler, pl.1, 1-7(?)

Brazil (Paraná)

LP

Remark: descr./ills. include cuticles

\section{Paraschizoneura Radchenko 1956}

P. sibirica (Nejburg 1948) Radchenko 1956

1980 Huang, pl.220, 1-4

China (Jilin)

UP

Parasphenophyllum Asama 1970

P. crenulatum Maithy 1978

1978 Maithy, pl.2, 11, 12

India (West Bengal)

UPRani

Parataxopitys Maniero 1951

P. americana Maniero 1951

1978 Mussa (a), fig.2E

(scheme from Maniero, 1951)

$\mathrm{P}$

\section{Paratordoxylon Mussa 1978}

P. camposi Mussa 1978

1978 Mussa (c), fig.3C

1978 Mussa (c), fig.6; pl.8, 49-51; pl.9, 52-55;

pl.10, 56-60; pl.11, 61-64

(rec.)

Brazil (São Paulo)

M-UP

\section{Paratrizygia Asama 1970}

P. rhodesii (Rigby 1966) Maithy 1978

1978 Maithy, pl.2, 10

India (-)

LPBara

\section{Partha Surange et Chandra 1973}

$P$. sp.

1977 Pant, fig.8D

P. belmontensis White 1978

1978 White, figs.36-40

1978 White, fig. 44

Remark: organic connection with cupulate seeds

$P$. indica (Surange et Maheshwari 1970) Surange et Chandra 1973 1977 Chandra et Surange (c), fig.2C

1980 Daber (a), fig.1C

$P$. sessilis White 1978

1978 White, fig. 41

1978 White, fig.44

Remark: organic connection with cupulate seed

$P$. spathulata Surange et Chandra 1973

1977 Chandra et Surange (c), fig.2B; pl.1, 3

Remark: in text $P$. spatulata

1978 Surange et Chandra, pl.2, 4

(rec. from Surange et Chandra, 1975)

Australia (New South Wales)

UP

(rec.)

(scheme)

(scheme)

Australia (New South Wales)

UP

(rec.)

India (-)

$P$

India (Orissa)

UPRani

\section{Pecopteris (Brongniart 1822) Sternberg 1825}

$P$. sp.

1976 Barthel (b), pl.3, 5

GDR (Karl-Marx-Stadt)

LPRotl

1976 Barthel et al., pl.11, 13

1976 Bohlin, figs.45-104, 105-165, 167-175, 180, 204, 259, 496, 500,

GDR (Karl-Marx-Stadt)

LPRotl

$503,504,506-513,517,532,533,535-539,542-544,546-549,551$,

$553-555,557,610,613,615,616,684-686,688,689,696,697,706$,

$712,718,721,725 ; \mathrm{pl} .4,3-5,7-9 ; 5-8$; pl.9, 1-2, 10-14;

pl.10, 1-3, 12; pl.11, 9; pl.12, 15; pl.28, 1, 2; pl.29, 3-5; pl.30, 1;

pl.31, 1, 2, 4, 5, 9, 10, 12-16,18, 19; pl.32, 19.20 (partly cf.?)

China (Kansu)

UC- $-\mathbf{L P}$ 
1976 Bohlin, figs.283, 284, 385, 455, 460, 468, 579-581,

$604,624,625,652,676 ;$ pl.17, $11 ;$ pl.21, $2 ;$ pl.26, 8

pl.31, 17; pl.32, 15 (partly cf.?)

1976 Lele (a), fig.4

1978 Cazzulo-Klepzig, fig.7B, C; pl.2, 5; pl.3, 4A, 5B

Remark: in text fig.7B,C; pl.8, 1A; pl.9, 1

1978 Daber et Helms, fig. p.36

1979 Kapoor, pl.170, 1

1979 Rösler, fig.4(2)

1979 Yuasa, fig.3.5

1980 El-Khayal et al., fig. $2 \mathrm{C}$

$P$. anbangensis Huang 1977

1977 Huang, fig.4; pl.5, 1-4; pl.27, 4

$P$. anderssonii Halle 1927

1976 Huang, pl.218, 3

1977 Feng et al., pl.243, 12

P. anthriscifolia (Göppert 1845) Zalessky 1918

1976 Durante, pl.22, 1-6 (aff.); pl.23, 2 (ex gr.)

1976 Huang, pl.216, 4, 4A

$P$. arborescens Sternberg 1825

1977 Feng et al., pl.242, 1, 2

1977 Krumbiegel et Walther, fig.2.96.12

1978 Gillespie et al., pl.31, 10; pl.32, 9

P. arcuata Halle 1927

1976 Huang, pl.218, 2, 2A

1980 Tian et Huang, pl.8, 1-3, 5; pl.9, 2, 3

P. bobrovii Bobrov et Nejburg 1957

1976 Durante, fig.28; pl.19, 1-4; pl.20, 1, 2

$P$. cambuhyensis Read 1941

Remark: in text $P$. cambuyensis

1979 Rösler, fig. 6

P. candolleana Brongniart 1828

1977 Krumbiegel et Walther, fig.2.96.15

1978 Gillespie et al., pl.31, 2

1978 Gillespie et al., pl.32, 3, 6

1980 Huang, pl.230, 18

+ P. cyathea (Schlotheim 1804) Brongniart 1828

1977 Huang, pl.3, 3 (cf.)

1978 Gillespie et al., pl.31, 11

1978 Zhang, pl.155, 3 (cf.)

1980 Huang, pl.228, 2-3A

$P$. deducans Huang 1977

1977 Huang, fig.5; pl.20, 2-3

P. densifolia (Göppert 1864) Weiss 1869

1980 Huang, pl.229, 5.6

$P$. dissimilaris Burago 1980

1980 Burago (b), fig.10; pl.13, 3

P. echinata $\mathrm{Gu}$ et $\mathrm{Zhi} 1974$

1978 Zhang, pl.155, 1

1980 Zhao et al., pl.8, 1, 1A

P. elegantula Zhang 1980

1980 Zhao et al., pl.9, 1--2B

+ P. feminaeformis (Schlotheim 1804) Sterzel 1880

1976 Huang, pl.215, 3, 4

1978 Gillespie et al., pl.31, 9; pl.32, 4

$P$. fuyuanensis Zhang 1980

1980 Zhao et al., pl.9, 3; pl.10, 1

+ P. gigantea (Schlotheim 1820) Brongniart 1828

1885 Quenstedt, pl.91. 17

\begin{tabular}{|c|c|}
\hline $\begin{array}{l}\text { China (Chinghai) } \\
\text { (scheme) }\end{array}$ & $\mathrm{UC}-\mathrm{LP}$ \\
\hline Brazil (Santa Catarina) & UPKaza-Tata \\
\hline (scheme) & $(\mathrm{C} P \mathrm{P})$ \\
\hline $\begin{array}{l}\text { India (Jammu and Kashmir) } \\
\text { (scheme) }\end{array}$ & $\mathrm{P}$ \\
\hline (scheme from Kon'no, 1970) & $(\mathrm{C}-\mathrm{P})$ \\
\hline Saudi Arabia ('Unayzah) & UC-LP \\
\hline China (Heilongjiang) & UP \\
\hline China (Inner Mongolia) & LP \\
\hline China (Guangdong) & UP \\
\hline Mongolia & UP \\
\hline China (Inner Mongolia) & UP \\
\hline $\begin{array}{l}\text { China (Guangdong) } \\
\text { (scheme) }\end{array}$ & UP \\
\hline USA (West Virginia) & $\mathrm{UC}-\mathrm{LP} ?$ \\
\hline China (Inner Mongolia) & LP \\
\hline China (Guizhou) & UP \\
\hline Mongolia & UP \\
\hline Brazil (Paraná) & LP \\
\hline $\begin{array}{l}\text { (scheme) } \\
\text { (scheme) }\end{array}$ & \\
\hline USA (West Virginia) & $\mathrm{UC}-\mathrm{LP} ?$ \\
\hline China (Liaoning) & $\mathrm{L} \cdot \mathrm{UP}$ \\
\hline China (Heilongjiang) & UP \\
\hline USA (West Virginia) & UC-LP? \\
\hline China (Guizhou) & UP \\
\hline China (Liaoning) & UC-LP \\
\hline China (Heilongjiang) & UP \\
\hline China (Liaoning) & LP \\
\hline USSR (Primorskiy Kray) & UP \\
\hline China (Guizhou) & UP \\
\hline China (Guizhou/Yunnan) & UP \\
\hline China (Guizhou/Yunnan) & UP \\
\hline $\begin{array}{l}\text { China (Inner Mongolia) } \\
\text { USA (West Virginia) }\end{array}$ & LP \\
\hline USA (West Virginia) & UC-LP? \\
\hline China (Guizhou/Yunnan) & UP \\
\hline GFR (Saarland) & LP \\
\hline
\end{tabular}


P. guizhouensis Chang MS ex Zhang 1978

1978 Zhang, pl.154, 4, 5

1980 Zhao et al., pl.8, 2-3; pl.10, 2-5

China (Guizhou) UP

Remark: in text $P$. guizhouensis Zhang 1980

$P$. hemitelioides Brongniart 1833

1977 Feng et al., pl.242, 4

1977 Krumbiegel et Walther, fig.2.96.16

1978 Gillespie et al., pl.31, 4

1978 Gillespie et al., pl.32, 8

1978 Zhang, pl.154, 1

1979 Vozenin-Serra, pl.2, 7, 8

1980 El-Khayal et al., fig.2F

P. heteropinna (Yabe et Shimakura 1940) Gu et Zhi 1974

1978 Zhang, pl.154, 3

$P$. hirta Halle 1927

Remark: in text $P$. hiata

1977 Feng et al., pl.240, 10

$P$. hongshanensis Huang 1977

1977 Huang, pl.2, 4; pl.3, 4-6; pl.4, 1-5

$P$. integra (Andrae 1849) Schimper 1869

1976 Barthel et al., pl.11, 9, 10

$P$. julii Radchenko 1936

1976 Durante, pl.24, 1-2 (cf.)

$P$. lamuriana Heer 1865

1977 Broutin, pl.2, 2

1978 Gillespie et al., pI.31, 8

$P$. lativenosa Halle 1927

1976 Huang, pl.216, 8

1978 Zhang, pl.154, 2

$P$. laxifolia Nejburg 1948

1976 Durante, pl.20, 6 (aff.)

P. leninskiensis (Khakhlov 1931) Radchenko 1936

1976 Durante, pl.57, 8 (cf.)

Remark: according to text $P$. leninskiensis Gorelova

$P$. lingulata Zhang 1980

1980 Zhao et al., pl.8, 4-6

$P$. longifolioides Zhang 1980

1980 Zhao et al., pl.7, 3-5A

$P$. marginata Gu et Zhi 1974

1978 Zhang, pl.153, 8, 9

1980 Tian et Zhang, pl.10, 1, 1A, 4, 4A; pl.11, 6

1980 Zhao et al., pl.7, 2

$P$. mentiens Sterzel 1880

1976 Barthel et al., pl.14, 22

$P$. monyi Zeiller 1888

1980 Barthel (b), pl.4, B-E

$P$. mucronata Durante 1976

1976 Durante, fig.29; pl.19, 5, 6

$P$. nervosa (Halle 1927) Wang 1979

1979 Wang, pl.1, 1-4

$P$. niamdensis Zalessky et Chirkova 1937

fa. makuchinae Burago 1976

1976 Burago, pl.7, 1, 2

$P$. norinii Halle 1927

1978 Zhang, pl.155, 2

P. opposita Cazzulo-Klepzig 1978

1978 Cazzulo-Klepzig, fig.6C; pl.1, 4; pl.3, 5A

Remark: in text fig.6C; pl.7, 1

China (Guizhou/Yunnan)

UP

China (Guangdong) , UP

(scheme)

(scheme)

USA (West Virginia)

China (Guizhou)

Laos (-)

Saudi Arabia ('Unayzah)

China (Guizhou)

(UC-LP?)

$\mathrm{UC}-\mathrm{LP}$ ?

UP

$P$

UC-LP

UP

China (Guangdong) UP

China (Heilongjiang) UP

GDR (Karl-Marx-Stadt) ～LPRotl

Mongolia UP

Spain (Sevilla)

USA (West Virginia)

UCStep?-LPAutu?

UC-LP?

China (Inner Mongolia) L-UP

China (Guizhou) UP

Mongolia UP

Mongolia UP

China (Guizhou/Yunnan) UP

China (Guizhou/Yunnan) UP

China (Guizhou) UP

China (Guizhou) UP

China (Guizhou/Yunnan) UP

GDR (Karl-Marx-Stadt) ～LPRotl

GDR (Gera) IPRot

Mongolia UP

China (Jiangsu) UP

USSR (Primorskiy Kray) UP

China (Guizhou) UP

Brazil (Santa Catarina) UPKaza-Tata

China (Inner Mongolia) LP 
1977 Feng et al., pl.240, 5

1978 Chen et al., pl.151, 4-5A

P. pectinata Bertrand 1927

1977 Sopeña et al., fig.4.3

P. pedrasica Read 1941

1978 Cazzulo-Klepzig, fig.7A; pl.2, 6

Remark: in text fig.7A; pl.7, $1 ;$ pl.8, $1 \mathrm{~B}$

$P$. pluckenetii Sternberg 1825

1977 Krumbiegel et Walther, fig.2.96.19

1977 Schweitzer, fig. 19C

1978 Strasburger, fig. 8021

$P$. plumsteadiae Huard-Moine 1964

1976 Le Roux, pl.1, 4 5 (cf.)

P. polymorpha Brongniart 1828

1976 Huang, pl.215, 2 2B

1978 Gillespie et al., pl.31, 5

1980 Tian et Zhang, pl.9, 1, 1A, 4, 6 (all cf.)

P. polypodioides (Presl 1838) Němejc 1948

Remark: in text P. polypodioides (Sternberg) Barthel

1979 Doubinger et Alvarez-Ramis, pl.1, 2, 2A

1980 Barthel (b), pl.A C; pl.8, C

$P$. potoniei (Němejc 1940) Remy et Remy 1959

1980 Barthel (b), pl.6; pl.7; pl.8, A, B

$P$. punicoides Zhang et Mo 1979

1979 Zhang et Mo, pl.2, 67

$P$. qingyunensis Zhang 1980

1980 Zhao et al., pl.11, 1. 1A

P. sahnii Hsü 1952

1978 Chen et al., pl.151, 6

1978 Zhang, pl.153, 2

1980 Huang, pl.232, 4, 4A

1980 Tian et Zhang, fig.18; pl.7, 2, 2A, 4; pl.8, 4, 4A

1980 Zhao et al., pl.11, 6, 6A

P. saraepontana (Stur 1885) Jongmans et Gothan 1935

1976 Bohlin, fig.505 (cf.)

P. shuichengensis Zhang 1978

1978 Zhang, pl.155, 6

P. singeri Archangelsky 1958

1976 Le Roux, pl.1, 13

P. taiyuanensis Halle 1927

1978 Zhang, pl.155, 5, (cf.)

$P$. tangwangheensis Huang 1980

1980 Huang, pl.258, 1 2A

$P$. tenuicostata Halle 1927

1978 Chen et al., pl.155, 4

P. tuberculata Halle 1927

1976 Huang, pl.216, 7

$P$. tumenjiangensis Huang 1980

1980 Huang, pl.231, 12 ; pl.232, 1-2A

P. unita Brongniart 1828

1977 Krumbiegel et Walther, fig.2.96.17

1978 Gillespie et al., pl.31, 3, 7

1978 Gillespie et al., pl.32, 5, 10

P. wongii Halle 1927

1976 Huang, pl.216, 13

1977 Feng et al., pl.240, 8

1980 Huang, pl.232, 3

P. yabei Kawasaki 1934

1977 Huang, pl.3, 1 2; pl.8, 6 7

1980 Huang, pl.233, 14
China (Guangdong) UP

China (Sichuan) UP

Spain (Guadalajara) LPAutu

Brazil (Santa Catarina) UPKaza-Tata

(scheme)

(rec.) (C P)

(scheme from Arnold) (C-P)

South Africa (Transvaal) LP

China (Inner Mongolia) L. UP

USA (West Virginia) UC LP?

China (Guizhou)

UP

France (Vosges) LPAutu

GDR (Gera) LPRotl

GDR (Gera) LPRotl

China (Henan) LP

China (Guizhou/Yunnan) UP

China (Sichuan) UP

China (Guizhou) UP

China (Liaoning) LP

China (Guizhou) UP

China (Guizhou/Yunnan) UP

China (Kansu) UC $\cdots$ LP

China (Guizhou) UP

South Africa (Transvaal) LP

China (Guizhou) UP

China (Heilongjiang) UP

China (Sichuan) UP

China (Inner Mongolia) LP

China (Jilin) UP

(scheme)

(scheme)

USA (West Virginia)

(UC-LP?)

UC LP?

China (Inner Mongolia) LP

China (Guangdong) UP

China (Liaoning) LP

China (Heilongjiang) UP

China (Jilin) UP 
Pelourdea Seward 1917

$P$. contracta Mo 1980

1980 Zhao et al., pl.21, 5-7

China (Guizhou/Yunnan)

UP

Peltaspermum Harris 1937

$P$. sp.

1979 Goman'kov et Meien, fig.4 (cuti); pl.14, 1-2, 3 (cuti)

USSR (Vologda - Aristovo -) $\quad$ UPTata

1980 Dobruskina, pl.22, 18-20

1980 Durante, fig. 1 ; pl.10, 1-6

USSR (Krasnoyarskiy Kray

- Tungusk Basin -) UP-LT

China (Xizang) UP

P. buevichae Goman'kov et Meien 1979

1979 Goman'kov et Meien, figs.3 (d: cuti), 5 (cuti);

pl.14, 4, 5-6 (cuti)

$P$. thomasii Harris 1937

1979 Dilcher, fig. 16

USSR (Vologda - Aristovo -) $\quad$ UPTata

(rec. from Townrow, 1960)

Peltostachys Barthel et al. 1976

$P$. altendorfense Barthel et al. 1976

1976 Barthel et al., fig.10; pl.28, 12-14, 15 (spore), 16

GDR (Karl-Marx-Stadt)

LPRotl

\title{
Petschoria Korde 1951
}

Remark: in text Petscheria. Petschoria is a Rhodophyceae genus $P$. sp.

1977 Huang, pl.17, 6

China (Heilongjiang)

UP

\section{Phasmatocycas Mamay 1973}

P. kansana Mamay 1973

1980 Bold et al., fig.24-41

(rec. from Mamay, 1976)

\section{Phylladoderma Zalessky 1914}

P. sp.

1976 Burago, fig.3; pl.7, 4-10

$P$. aequalis Meien 1977

1977 Meien, fig.5; pl.8, 5-10

Remark: descr./ills. include cuticles

P. arberi Zalessky 1914

1976 Bohlin, figs.17-22; pl.3, 7 (all cf.)

$P$. stenuifolia Sal'menova 1978

1978 Sal'menova, pl.12, 7, 8

\author{
USSR (Primorskiy Kray) UP \\ USSR (Vologda - Aristovo - ) UPTata \\ China (Kansu) \\ UC-LP \\ USSR (Kazakhstan) \\ UP
}

\section{Phyllocladopitys Kräusel 1928}

$P$. capensis Kräusel 1928

1978 Mussa (a), fig.2D

(scheme from Kräusel)

Remark: in text $P$. capense and $P$. capensis

\section{Phyllotheca Brongniart 1828}

P. sp.

1976 Bohlin, fig.10 (cf.)

China (Kansu)

UC-LP

1979 Asama, fig.13C

(scheme from Rasskazova, 1961)

(scheme)

$P$. australis Brongniart 1828

1979 Millan et Dolianiti, fig.1

1979 Van Dijk et al., pl.45, 1

1980 Retallack, figs.216.5A-C

1980 Rigby et al., fig.1

Brazil (São Paulo)

South Africa (Natal)

Australia (New South Wales)

Australia (New South Wales) 
P. equisetitoides Schmalhausen 1879

Remark: in text $P$. equisetoides

1980 Bold et al., fig. $19-57$

(rec. from Rasskazova)

(P-T)

$P$. etheridgei Arber 1905

1978 Zhang, pl.152, f.5 (cf.)

+ P. turnaensis Gorelova 1956 ex Gorelova et Radchenko 1962?

1978 Durante et Biterman, pl.23, 8; pl.24, f.5

Remark: in text P. turnaensis (Gorelova) Meien

China (Guizhou)

UP

USSR (Yakutskaya ASSR -Verkhoyansk -)

UP

\section{Plagiozamites Zeiller 1894}

P. sp.

1976 Bohlin, figs.24, 35, 273; pl.2, 3; pl.3, 9 (all cf.?)

1976 Bohlin, figs.281, 298; pl.29, 11-12 (all ?)

1978 Remy et Remy (a), pl.16, 6, 7

1978 Zhang, pl.153. 5

P. linearis Yabe et Oishi 1938

1978 Zhang, pl.153, 6 7 (cf.)

P. oblongifolius Halle 1927

1976 Bohlin, fig.23; pl.2, 5 8; pl.3, 5 (all cf.)

1977 Feng et al., pl.238, 5

1978 Zhang, pl.152, 4

1980 Zhao et al., pl.17, 5; pl.20, 3

P. planchardii (Renault 1890) Zeiller 1894

1977 Remy et Remy, fig.75A-B (cf.)

1978 Remy et Remy (a), fig.2; pl.16, 4-5 (all cf.)

$\begin{array}{ll}\text { China (Kansu) } & \text { UC -.-LP } \\ \text { China (Chinghai) } & \text { UC--LP } \\ \text { GDR (Halle) } & \text { LPAutu } \\ \text { China (Guizhou) } & \text { UP } \\ \text { China (Guizhou) } & \text { UP } \\ \text { China (Kansu) } & \text { UC-LP } \\ \text { China (Henan) } & \text { LP } \\ \text { China (Guizhou) } & \text { UP } \\ \text { China (Guizhou/Yunnan) } & \text { UP } \\ \text { GDR (Halle) } & \\ \text { GDR (Halle) } & \text { LPAutu } \\ \end{array}$

\section{Platycardia Pant et Nautiyal 1960}

P. sp.

1977 Pant, fig. $7 \mathrm{~A} C$

P. bengalensis Pant et Nautiyal 1960

1977 Chandra et Surange (e), fig.5; pl.3, 16-18

1977 Chandra et Surange (e), fig.7

Remark: descr./ills. include cuticles

1977 Pant, fig.6B

(rec.)

India (West Bengal) UPRani

(rec.)

(from Pant et Nautiyal, 1960)

\section{Pluma Plumstead 1958}

P. sp.

1977 Pant, fig.9D,E

(from Plumstead, 1958)

\section{Plumsteadia Rigby 1963}

$P$. sp.

1977 Pant, fig.9G

1979 Van Dijk et al., pl.46, 27-30 (cf.)

P. ampla (White 1964) Rigby 1969

1978 Rigby (a), figs. 512

P. lanceolata Srivastava 1978

1978 Srivastava, fig. $4 ; \mathrm{pl} .1,4,5$

P. microsacca Rigby 1963

1976 Gould, fig.4B

1978 Rigby (a), figs.2, 3

$P$. natalensis Lacey, Van Dijk et Gordon-Gray 1975

1979 Van Dijk et al., pl.46, 26

$P$. ovata Kyle 1974

1978 Rigby (a), fig.13(?)

$P$. semnes Rigby 1978

1978 Rigby (a), figs. $14 \cdot 17$

(from Plumstead, 1958)

South Africa (Natal)

UP

Australia (Queensland)

UP

India (Bihar)

UPRani

(scheme from Rigby, 1971)

Australia (Queensland)

UP

South Africa (Natal)

UP

Australia (Queensland)

UP

Australia (Queensland)
LP 
$P$. sp.

1979 Chandra et Surange, pl.22, 7

India (West Bengal)

LGRani

Remark: organic connection with Glossopteris gondwanensis P. ellipticus Chandra et Surange 1977

1977 Chandra et Surange (a), figs.2-5; pl.1, 1-4; pl.2,

$5-8 ; \mathrm{pl} .3,9,12-13 ; \mathrm{pl} .4,14-16 ; \mathrm{pl} .5,18 ; \mathrm{pl} .6,21$

1977 Chandra et Surange (a), figs.6, 7

Remark: descr./ills. include cuticles.

Organic connection with Glossopteris taenioides

1977 Chandra et Surange (e), fig.14B

Remark: organic connection with Glossopteris taenioides

1978 Surange et Chandra, pl.4, 4

1979 Chandra et Surange, pl.13, 3, 6

Remark: organic connection with Glossopteris gondwanensis

$P$. pretiosus Chandra et Surange 1977

1977 Chandra et Surange (c), fig.2E

India (West Bengal)

LGRani

(rec.)

(rec.)

(rec.)

India (West Bengal)

LGRani

(scheme)

\section{Poa-Cordaites Grand'Eury 1872}

Remark: commonly spelled Poacordaites

P. sp.

1976 Barthel (b), pl.2, 14 (cuti)

1976 Barthel et al., pl.45, 1A-D (cuti)

1977 Krumbiegel et Walther, fig.2.100.1

1979 Doubinger et Alvarez-Ramis, pl.1, 3

GDR (Karl-Marx-Stadt)

GDR (Karl-Marx-Stadt)

(rec. from Grand'Eury)

France (Vosges)

Brazil (São Paulo)

Brazil (Rio Grande do Sul)

Brazil (Rio Grande do Sul)

Brazil (Rio Grande do Sul)

LPRotl

LPRotl

LPAutu

(from Nejburg, 1960)

Brazil (Santa Catarina)

LPArti-Kung

France (Saône-et-Loire)

LP

(scheme from Chauveau)

China (Guizhou/Yunnan)

UP
$\mathrm{P}$

$\mathrm{P}$

$\mathbf{P}$

$\mathrm{P}$

\section{Polyssaievia Nejburg 1960}

1979 Lacey, fig.1(6)

\section{Ponsotheca Bernardes de Oliveira 1980}

$P$. roesleri Bernardes de Oliveira 1980

\section{Poroxylon Renault 1879}

1977 Marguerier, pl.1, 2

P. edwardsii Renault

1978 Mussa (a), fig.1J

\section{Prionophyllopteris Mo 1980}

$P$. spiniformis Mo 1980

1980 Zhao et al., pl.19, 9, 10

\section{Protoblechnum Lesquereux 1880}

P. wongii Halle 1927

1976 Asama, fig.1(11)

(scheme) 
1977 Broutin, pl.1, 1 2; pl.3, A-B (all cf.)

1979 Asama, fig.3(11)

1980 Asama, fig.5

\section{Protophyllocladoxylon Kräusel 1939}

P. sp.

1977 Marguerier, pl.2, 6, 7

Protopodocarpitys Mussa 1976

P. roesleri Mussa 1976

1976 Mussa, figs.1 6; pls.1 5

\section{Protosphagnum Nejburg 1960}

P. nervatum Nejburg 1956 ex Nejburg 1960

1978 Fefilova (b), fig.21; pls.21, 22

1979 Lacey, fig.1(1, 4)

\section{Prynadaeopteris Radchenko 1955}

P. anthriscifolia (Göppert 1845) Radchenko 1955

1977 Huang, pl.1, 3 6; pl.2, 1 3

$P$. minuta Vladimirovich 1980

1980 Vladimirovich, pl.10, 3(?)

P. rhomboidea Vladimirovich 1980

1980 Vladimirovich, pl.10, 12 2(?)

P. tunguscana (Schmalhausen 1879) Radchenko 1955

1979 Burago, pl.6, 5; pl.7, 2, 3; pl.8, 4

\section{Psaliangium Remy 1953}

Remark: in text Psalangium

$P$. sp.

1976 Bohlin, fig. 176

\section{Psaronius Cotta 1832}

P. sp.

1976 Grzimek, fig.p.52

1977 Krumbiegel et Walther, pl.6, 11

1977 Remy et Remy, fig. 53

1977 Remy et Remy, fig.55C

1978 Gillespie et al., fig.11

1979 Asama, fig.21A

1979 Steel et Harvey, fig. p.38

1979 Yuasa, fig.3.4

1980 Barthel et Fischer, figs.1, 2

1980 Bold et al., fig. 19 11

1980 Bold et al., fig. 1965

1980 Tian et Zhang, pl.24, 1, 1A, 1B

$1980 \mathrm{X}$. number " 4 " in fig.

P. asterolithus Cotta 1832

1885 Quenstedt, pl.93, 9

P. brasiliensis Brongniart 1872

1976 Fischer et Gayrard-Valy, figs.2, 39

$P$. hexagonus Gu et Zhi 1974

1978 Chen et al., pl.154, 5

$P$. infarctus Unger 1841

1976 Rothe, fig. 10
Namibia (--)

K

Brazil (São Paulo)

P

Spain (Sevilla)

(scheme)

(scheme)

UCStep?-LPAutu?

USSR (Komi ASSR)

(from Nejburg, 1960)

LPUfim

China (Heilongjiang)

UP

USSR (Komi ASSR - Pechora

Basin -..)

UP

USSR (Komi ASSR Pechora Basin ---)

UP

USSR (Primorskiy Kray)

LP

China (Kansu)

UC-LP

$\mathrm{P}$

GDR (Karl-Marx-Stadt) LPRotl

GDR (Karl-Marx-Stadt) LPAutu

GDR ("Sachsen")

LPAutu

(C-P)

(C-P)

(C-P)

(C-P)

LPRotl

$\mathrm{C}-\mathrm{P}$

$(\mathrm{C}-\mathrm{P})$

UP

China (Guizhou)

(rec. from Morgan, 1959)

GDR (Karl-Marx-Stadt)

LP

Brazil (Piaui)

$\mathrm{p}$

China (Sichuan)

UP

GDR (Karl-Marx-Stadt)

LPRotl 
1977 Krumbiegel et Walther, pl.51, 85

1978 Daber et Helms, fig. p.174

1978 Daber et Helms, fig. p.191

P. polyphyllus Feistmantel 1873

1976 Barthel et al., pl.11, 14

$P$. simplex Unger 1841

1976 Daber et Helms, fig. p.73

$P$. sinensis Sze 1942

1978 Zhang, pl.162, 1-3

$P$. ungeri Corda 1845

1976 Barthel et al., pl.11, 11

$P$. weberi Sterzel 1887

1977 Krumbiegel et Walther, pl.18, 31

\section{+ Pseudomariopteris Danzé-Corsin 1953}

$P$. cordato-ovata

1978 Gillespie et al., pl.49, 4

Remark: probably new combination?

P. ribeyronii (Zeiller 1888) Danzé-Corsin 1953

1976 Barthel et al., pl.31, 1-3

\section{Pseudorhipidopsis P'an 1937}

P. brevicaulis (Kawasaki et Kon'no 1932) P'an 1937

1977 Feng et al., pl.250, 5

\section{Pseudovoltzia Florin 1927}

P. sp.

1977 Schaumberg, fig.14(?)

1979 Yuasa, fig.5.8

P. liebeana (Geinitz 1862) Florin 1927

1976 Harris, fig.1D-L

1977 Krumbiegel et Walther, fig.2.103.2-5

1977 Remy et Remy, fig. 45

1977 Schaumberg, fig.12

1977 Vogellehner (b), fig.3A

1978 Grauvogel-Stamm, fig.45

1978 Miller, fig.2A,B

\section{Psygmophyllum Schimper 1870}

P. kidstonii Seward 1903

1976 Le Roux, pl.3, 11, 12

P. multipartitum Halle 1927

1976 Asama, fig.1(12)

1977 Feng et al., pl.253, 1, 2

1979 Asama, fig.3(12)

1980 Asama, fig.5

P. ussuriense Burago 1976

1976 Burago, pl.7, 3

\section{+ Pterispermostrobus Stopes 1914}

Remark: in text Pteridospermostrobus.

See Farr et al. (1979, p.1453)

$P$. wanderianus Florin

1976 Barthel et al., pl.16, 13

\section{Pterophyllum Brongniart 1825}

$P$. blechnoides Sandberger 1864

1977 Remy et Remy, fig.70A, B

$\begin{array}{ll}\begin{array}{l}\text { GDR (Karl-Marx-Stadt) } \\ \text { GDR (Karl-Marx-Stadt) } \\ \text { (scheme) }\end{array} & \begin{array}{l}\text { LPRotl } \\ \text { LPRotl }\end{array} \\ \text { GDR (Karl-Marx-Stadt) } & \text { LPRotl } \\ \text { GDR (Karl-Marx-Stadt) } & \text { LP } \\ \text { China (Guizhou) } & \text { UP } \\ \text { GDR (Karl-Marx-Stadt) } & \text { LPRotl } \\ \text { GDR (Karl-Marx-Stadt) } & \text { LPRotl }\end{array}$

USA (West Virginia)

UC-LP?

GDR (Karl-Marx-Stadt)

LPRotl

China (Henan)

UP

GFR (Hessen)

UPKupf

(scheme from Florin, 1944)

(scheme)

(from Mägdefrau)

GFR (Hessen)

GFR (Hessen)

(from Schweitzer, 1968)

(scheme from Schweitzer, 1963)

(rec. from Schweitzer, 1963)

South Africa (Transvaal)

LP

(scheme)

China (Henan)

UP

(scheme)

(scheme)

USSR (Primorskiy Kray)

UP

GDR (Dresden)

LPRotl

GFR (Baden-Württemberg)

LPRotl 
$P$. cotteanum Gutbier 1835 1976 Barthel (b), pl.4, 12 1976 Barthel et al., pl.38, 11 P. daihoense Kawasaki 1931 1976 Huang, pl.224, 3, 4

P. eratum Gu et Zhi 1974

1978 Zhang, pl.162, 4

1980 Huang, fig.42; pl.253, $2.5 \mathrm{~A}$

1980 Zhao et al., pl.20, 1 2

P. fromagetii Vozenin-Serra 1979

1979 Vozenin-Serra, pl.1, 13

GDR (Dresden)) LPRotl

GDR (Dresden) LPRotl

$+P$. slobodskiensis Shvedov

1977 Huang, pl.14, 5 (cf.)

China (Inner Mongolia)

L-UP

China (Guizhou)

UP

China (Liaoning)

UP

China (Guizhou/Yunnan)

UP

$\operatorname{Laos}()$

$P$

China (Heilongjiang)

UP

\section{Pterygospermum Pant et Nautiyal 1960}

P. raniganjense Pant et Nautiyal 1960

1977 Chandra et Surange (a), figs.2B, E G, 3A-B, E, 4A, E F, 5; pl.2, 5; pl.4, 17; pl.5, 18 20; pl.6, 21 26

India (West Bengal)

LGRani

Remark: descr./ills. include cuticles.

Organic connection with Plumsteadiostrobus ellipticus

Containing Faunipollenites pollen grains

1977 Pant, fig. $6 \mathrm{C}$

(from Pant et Nautiyal, 1960)

\section{Ptychocarpus Weiss 1869}

P. sp.

1977 Remy et Remy, fig.56C

P. malaianus Konno 1970

1979 Asama, fig.17B

P. unitus (Brongniart 1828) Zeiller 1888

1977 Remy et Remy, fig.124C

1978 Daber et Helms, fig. p.36

(scheme)

(C P)

Malaysia ( )

$\mathrm{P}$

(scheme)

(C $P$ )

(scheme)

(C $\mathrm{P})$

Pursongia Zalessky 1933

P. sp.

1980 Durante, pl.10.7(?), 8-10

China (Xizang)

UP

\section{Quadrocladus Mädler 1957}

Q. sp.

1978 Remy et Remy (c), pl.8, 21(?)

Italy (Brescia)

LPSaxo

Q. duinensis Meien 1978

1978 Meien, figs.14 19; pl.2, 13, 14

USSR (Vologda -- Aristovo...) UPTata

\section{Radicites Potonié 1893}

Remark: substitute name for Pinnularia

Lindley et Hutton 1833, non Ehrenberg 1843

(nom.cons.). See Farr et al. (1979, p.1480)

$R . \mathrm{sp}$.

1977 Huang, pl.27, 3

China (Heilongjiang)

UP

Rajahia Kon'no 1970

R. guizhouensis Zhang 1980

1980 Zhao et al., pl.11, 24

$R$. linggiuensis Kon'no 1970

1979 Asama, fig.17A

China (Guizhou/Yunnan)

UP

$R$. major Zhang 1980

1980 Zhao et al., pl.12, 2

Malaysia ( )

$\mathrm{P}$

China (Guizhou/Yunnan)

UP 
$R$. minor Zhang 1980

1980 Zhao et al., pl.12, 3-4A

China (Guizhou/Yunnan) UP

$R$. mirabilis (Gu et Zhi 1974) Zhang 1980

1980 Zhao et al., pl.11, 5, 5A

China (Guizhou/Yunnan) UP

$+R$. rigida (Yabe et Oishi) Zhang 1980

1980 Zhao et al., pl.12, 1, $1 \mathrm{~A}$

China (Guizhou/Yunnan)

UP

\section{Raniganjia Rigby 1963}

R. qubuensis Hsü 1976

1976 Hsü, fig.1; pl.1, 4-7; pl.4, f.25

China (Xizang)

UP

1978 Hsü, pl.2, 2

China (Xizang)

UP

\section{Renaultia Zeiller 1883}

$R$. sp.

1977 Remy et Remy, fig.61

(scheme)

(C-P)

\section{Reticulopteris Gothan 1941}

R. germari (Giebel 1857) Gothan 1941

1976 Barthel (b), pl.1, 2

1976 Barthel et al., pl.32, 5 (cuti), 6-9

GDR (Karl-Marx-Stadt)

LPRot]

GDR (Karl-Marx-Stadt)

LPRotl

\section{Rhabdocarpos Göppert et Berger 1848}

Remark: commonly spelled Rhabdocarpus. See Farr et al. (1979: 1498).

$R$. sp.

1976 Bohlin, pl.21, 12 (cf.)

R. olivaeformis Mo 1980

1980 Zhao et al., pl.17, 1

China (Chinghai)

UC-LP

China (Guizhou/Yunnan)

UP

\section{Rhabdotaenia Pant 1958}

$R$. sp.

1976 Lele (b), fig. 2

(scheme)

\section{Rhacopteris Schimper 1869}

$R$. sp.

1976 Bohlin, pl.27, 9(?)

$R$. bertrandii Stockmans et Mathieu 1939

1976 Huang, pl.211, 1

China (Kansu)

China (Inner Mongolia)

USSR (Komi ASSR)
UC-LP

LP

UPKaza

R. praecursoria Meien 1979

1979 Meien, figs. 1-4; pl.1, 1-8; pl.2, 1-8

Remark: descr./ills. include cuticles

\section{Rhipidopsis Schmalhausen 1879}

$R . \mathrm{sp}$.

1980 Tian et Zhang, pl.21, 6

$R$. ginkgoides Schmalhausen 1879

1978 Zhang, pl.163, 2 (cf.), 7

1980 Zhao et al., pl.21, 1-2 (cf.)

$R$. gondwanensis Seward 1919

1979 Chitnis et Vagyani, fig. 3 ; pl.1, 2

1980 Tian et Zhang, pl.19, 1, 2; pl.22, 6, 11

R. guizhouensis Tian 1980

1980 Tian et Zhang, pl.14, 1, 2, 6

$\begin{array}{ll}\text { China (Guizhou) } & \text { UP } \\ \text { China (Guizhou) } & \text { UP } \\ \text { China (Guizhou/Yunnan) } & \text { UP } \\ \text { India (Maharashtra) } & \text { UP-LT } \\ \text { China (Guizhou) } & \text { UP } \\ \text { China (Guizhou) } & \text { UP }\end{array}$


R. hongshanensis Huang 1977

1977 Huang, fig.19: pl.10, 4

R. lobulata Mo 1980

1980 Zhao et al., pl,19, 11, 12

China (Heilongjiang)

UP

R. minor Feng 1977

1977 Feng et al., pl.250. 2

China (Guizhou/Yunnan)

UP

China (Hunan) UP

China (Guizhou) UP

R. multifurcata Tian 1980

1980 Tian et Zhang, pl.23, 2, 2A

China (Guizhou) UP

$R$. palmata Zalessky 1932

China (Guizhou) UP

1976 Durante, pl.52, 2 (aff.)

Mongolia

UP

$+R$. panii Chow

1977 Feng et al., pl.251, 6

1978 Chen et al., pl.154, 1 2; pl.155, 12

China (Guangdong)

UP

China (Sichuan) UP

China (Guizhou) UP

China (Guizhou) UP

1980 Tian et Zhang, fig.22; pl.17, 4

China (Guizhou/Yunnan) UP

China (Heilongjiang) UP

1980 Huang, fig.44; pl.258, 6-7

$R$. shuichengensis Tian 1980

1980 Tian et Zhang, fig.23; pl.20, 1-6; pl.21, 1--3

$R$. tangwangheensis Huang 1980

1980 Huang, fig.43; pl.253, $6 ;$ pl.255, 9

China (Guizhou)

UP

China (Heilongjiang)

UP

$R$. xinganensis Huang 1977

1977 Huang. fig. 18; pl.17, 4; pl.29, 4.5

China (Heilongjiang)

UP

\section{Rhizomopsis Gothan et Sze 1933}

$R$. gemmifera Gothan et Sze 1933

1978 Zhang, pl.164. 7

1980 Tian et Zhang, pl.22, 7, 8, 12

1980 Zhao et al.. pl.20. 5

China (Guizhou)

China (Guizhou)

UP

China (Guizhou/Yunnan)

$\mathrm{UP}$

UP

\section{Rhodea Presl 1838}

R. sp.

1976 Bohlin, figs.391 395; pl.21, 3, 6 7, 8 (all cf.)

China (Chinghai)

UC LP

\section{Ricciopsis Lundblad 1954}

R. anakitensis Verbitskaya 1980

1980 Verbitskava (a), fig. 2 ; pl.4, 34 (all ?)

R. parva Verbitskaya 1980

1980 Verbitskaya (a). fig. 3 ; pl.4, 5 . 6 (all ?)

USSR (Krasnoyarskiy Kray

USSR (Kemerovskaya oblast ---

Kuzbass -..)

UP

Rigbya Lacey, Van Dijk et Gordon-Gray 1975

R. arberioides Lacey. Van Dijk et Gordon-Gray 1975

1978 Rigby (a), figs.22, 23

1978 Surange et Chandra, pl.2, 5

1978 White, figs. 6972

Remark: organic connection with seeds

1979 Van Dijk et al.. pl.46, 1719

$\begin{array}{ll}\text { Australia (Queensland) } & \text { UP } \\ \text { South Africa (Natal) } & \text { UP } \\ \text { Australia (New South Wales) } & \text { UP } \\ & \\ \text { South Africa (Natal) } & \text { UP }\end{array}$

Rubidgea Tate 1867

$R . \mathrm{sp}$.

1976 Lele (b), fig. 2

1977 Schweitzer, fig.8C

1980 Andreis et al., pl.1. 2

(scheme)

(rec.)

Brazil (Rio Grande do Sul) LP 
1980 Cazzulo-Klepzig et al. (a), pl.2, 1; pl.3, 5

1980 Cazzulo-Klepzig et al. (b), pl.1, 3, 8; pl.2, 2

1980 Guerra-Sommer, pl.1, 8, 10

$R$. emarginata Maithy 1977

1977 Maithy (c), pl.1, 4, 5

\section{Rufloria Meien 1963}

$R$. sp.

1976 Durante, pl.61, 6

1976 Lele (a), fig. 4

1977 Zimina, pl.13, 6

1980 Goman'kov et Meien, fig.1(3)

R. bakanasica Sal'menova 1978

1978 Sal'menova, pl.12, 4-6

R. brevifolia (Gorelova 1960) Meien 1966

1976 Durante, pl.65, 1-4

R. delicata Durante 1971

1976 Durante, pl.45, 5; pl.63, 1-5, 6 (aff.); pl.64, 9, 10; pl.74, 3

Remark: descr./ills. include cuticles

R. derzavinii (Nejburg 1936) Meien 1966

1976 Durante, pl.60, 1-3 (aff.), 4-7

1976 Zimina (b), pl.5, 6, 7; pl.6, 1-5; pl.7, 1 (all aff.)

1977 Zimina, fig.17; pl.12, 1-4; pl.13, 1-5, 7-8 (aff.);

pl.14, 1-8 (aff.)

R. ilimpejica Glukhova 1976

1976 Glukhova, fig.1; pl.10, 4-6

R. kerulenica Durante 1976

1976 Durante, pl.59, 1-7

R. lepeophylloides Durante 1976

1976 Durante, pl.65, 5-12

R. magnifolia Durante 1976

1976 Durante, pl.62, 1-8

R. marginata Men'shikova 1980

1980 Glukhova et Men'shikova

R. mira Glukhova et Men'shikova 1980

1980 Glukhova et Men'shikova, pl.14, 6-9

R. mirabilis Meien 1966

1977 Zimina, pl.7, 9 (aff.)

R. rasskasovae Meien 1966

1976 Durante, pl.58, 3-5

R. recta (Nejburg 1965) Meien 1966

1977 Zimina, pl.15, 1-8 (aff.)

$R$. seljatitskii Men'shikova 1980

1980 Glukhova et Men'shikova, pl.14, 3-5

R. sublanceolata Zimina 1976

1976 Zimina (b), pl.5, 5

1977 Zimina, pl.10, 9; pl.15, 9

1978 Zimina, pl.2, 5

$R$. theodorii (Chirkova et Zalessky 1930) Meien 1964

1977 Zimina, fig.18; pl.7, 3-8 (all aff.)

1978 Zimina, pl.1, 1-4 (aff.)

R. tomentella Glukhova 1976

1976 Glukhova, pl.10, 1-3

R. tuberculosa Glukhova 1971

1976 Durante, pl.61, 1-5
Brazil (Rio Grande do Sul)

Brazil (Rio Grande do Sul)

LPSakm

LPSakm

LPSakm

India (Bihar)

UPKarh

Mongolia

LP

(scheme)

USSR (Primorskiy Kray)

LP

(scheme)

USSR (Kazakhstan)

LP

Mongolia

UP

Mongolia

UP

Mongolia

LP

USSR (Primorskiy Kray)

L-UP

USSR (Primorskiy Kray)

LP

USSR (Krasnoyarskiy Kray Tungusk Basin - )

UP

Mongolia

LP

Mongolia

UP

Mongolia

UP

USSR (Kemerovskaya oblast Kuzbass -)

UP

USSR (Kemerovskaya oblast Kuzbass -)

UP

USSR (Primorskiy Kray)

LP

Mongolia LP

USSR (Primorskiy Kray) LP

USSR (Kemerovskaya oblast Kuzbass -)

UP

USSR (Primorskiy Kray)

USSR (Primorskiy Kray)

USSR (Primorskiy Kray)

L-UP

LP

LP

USSR (Primorskiy Kray) LP

USSR (Primorskiy Kray)

LP

USSR (Krasnoyarskiy Kray - Tungusk Basin -)

UP

Mongolia

LP 
R. ulannurica Durante 1976

1976 Durante, pl.63, 7-10, 11 (aff.); pl.64, 1-2 (aff.),

3-4 (cuti), 6-8 (aff.); pl.74, f.1 (cuti)

Mongolia

UP

R. ussurica Zimina 1977

1977 Zimina, figs.19, 20; pl.8, 1-6, 7(?); pl.9, 2, 3;

pl.10, 1-2 (ex gr.), 3; pl.11, 4 - 7 (ex gr.), 8, 9

1978 Zimina, pl.1, 5; pl.2, 4, 7

R. vassilyevii Zimina 1977

1977 Zimina, pl.16, 1 3(?), 4 (ex gr.)

USSR (Primorskiy Kray)

LP

USSR (Primorskiy Kray)

LP

USSR (Primorskiy Kray)

$\mathrm{UP}$

\section{Rusangea Lacey, Van Dijk et Gordon-Gray 1975}

Remark: in text Russangea

R. elegans Lacey, Van Dijk et Gordon-Gray 1975

1978 Surange et Chandra, pl.3, 5

1978 White, figs. 42,43

1978 White, fig. 44

Remark: organic connection with seeds

South Africa (Natal

UP

Australia (New South Wales)

UP

\section{Sakoarota Appert 1977}

S. polyangiata Appert 1977

1977 Appert, figs.2, 3; pl.10, 2-4, 11-17; pl.18,

1 . 7,$9 ; \mathrm{pl} .19,1-3$ (cf.) $4 ; \mathrm{pl} .20 ; \mathrm{pl} .21$

Madagascar (Sakoa Basin)

LGKarh-Bara?

\section{+ Sagittapteris Zhang et Xiao}

+ S. belemnopteroides Zhang et Xiao

Remark: paper by Zhang et Xiao not studied by the compilers of the IFPM

1980 Zhang, pl.1, 36

China (Shanxi)

$\mathrm{P}$

\section{Salairia Nejburg 1960}

S. confinis Fefilova 1976

1978 Fefilova (b), fig.7; pl.6

S. singularis Fefilova 1976

1978 Fefilova (b), fig.8; pl.7

USSR (Komi ASSR)

LPUfim

USSR (Komi ASSR)

LPUfim

\section{Samaropsis Göppert 1864}

S. sp.

1976 Bohlin, fig.231; pl.15, 13

1976 Durante, pl.72, 13, 14

1977 Bernardes de Oliveira et Da Silva Pontes,

figs. 15,16 ; pl.5. 4,5

1978 Zhang, pl.164, 6

1980 Guerra-Sommer, pl.1, 6

1980 Rigby et al., fig. 52

1980 Tian et Zhang, fig.24; pl.19, 3, 4

S. dolianitii Millan 1977

1977 Millan, pl.3, 2-5

S. ganjrensis Saksena 1950 ex Saksena 1955

1977 Srivastava (a), fig.8; pl.1, 5

$S$ goraiensis Surange et Lele 1957

1980 Rigby et al., fig.51 (cf.)

+ S. kureikaensis Sukhov

1976 Durante, pl.72, 1

China (Kansu)

Mongolia

UC-LP

LP

$\begin{array}{ll}\text { Brazil (Santa Catarina) } & \text { LPArti } \\ \text { China (Guizhou) } & \text { UP } \\ \text { Brazil (Rio Grande do Sul) } & \text { LPSakm } \\ \text { Australia (New South Wales) } & \text { P } \\ \text { China (Guizhou) } & \text { UP } \\ \text { Brazil (São Paulo) } & \text { LP } \\ \text { India (Bihar) } & \text { LGKarh } \\ \text { Australia (New South Wales) } & \text { P } \\ \text { Mongolia } & \text { UP } \\ & \\ \text { Brazil (Santa Catarina) } & \text { L.PArti } \\ \text { Brazil (São Paulo) } & \text { LP }\end{array}$

1977 Bernardes de Oliveira et Da Silva Pontes,

fig. 16; pl.5, 13

Brazil (São Paulo)

LP 
S. sancti-marci Bernardes de Oliveira 1977

1977 Bernardes de Oliveira et Da Silva Pontes,

figs.17-23; pl.5, 6-8

S. seixasi (White 1908) Seward 1917

1977 Correa da Silva et Arrondo, pl.2, 6, 7

1978 Correa da Silva, pl.5, 2

1980 Cazzulo-Klepzig et al. (a), pl.3, 4

1980 Cazzulo-Klepzig et al. (b), pl.1, 1

S. taiyuanensis Halle 1927

1978 Chen et al., pl.153, 6

1978 Mamay, pl.1, 1-3 (cf.), 4, 5-6 (cf.)

Remark: new ills. of the holotype

\section{Santhalea Maithy 1977}

S. bansloiensis Maithy 1977

1977 Maithy (b), fig.2; pl.2, 3-6

\section{Sashinia Meien 1978}

S. aristovensis Meien 1978

1978 Meien, figs.11, 12, 13 (cuti); pl.2, 15

\section{Schimperia Remy et Remy 1975}

S. sp.

1977 Remy et Remy, fig.234B

\section{Schizoneura Schimper et Mougeot 1844}

Remark: correct genus name Convallarites Brongniart 1828. See Farr et al. (1979, p.1572)

S. sp.

1976 Balasundaram et al., pl.22, 1

1976 Bohlin, fig.619; pl.17, 6

1979 Asama, fig.13F

1979 Asama, fig.13G

1979 Asama, fig. $13 \mathrm{H}$

1979 Yuasa, fig.4.7

1979 Yuasa, fig.4.8

S. brevifolia Yao 1980

1980 Zhao et al., pl.4, 8, 9, 9A

S. gondwanensis Feistmantel 1876

1977 Bose et al., pl.1, 1

1978 Cazzulo-Klepzig, fig.3A-D; pl.1, 1-3

Remark: in text fig. $3 ;$ pl.2, $1-2 ;$ pl.3, $1-2$

1979 Van Dijk et al., pl.46, 13, 14

S. lingshanensis Feng et Meng 1977

1977 Feng et al., pl.239, 1

S. manchuriensis Kon'no 1942

1977 Huang, pl.12, 4-5 (cf.)

1978 Chen et al., pl.151, 3

1980 Huang, pl.242, 1-5

1980 Zhao et al., pl.4, 4 (cf.), 5

\section{Schizostachys Grand'Eury 1877}

S. spiciformis (Andrae 1851) Barthel 1975

1980 Barthel (c), pl.8, 2

Brazil (Santa Catarina)
Brazil (Santa Catarina)

Brazil (Rio Grande do Sul)

Brazil (Rio Grande do Sul)

Brazil (Rio Grande do Sul)

Brazil (Rio Grande do Sul)

China (Sichuan)

USA (Texas)

LP

LPArti

LPSakm-Arti

LPSakm

LPSakm

LPSakm

UP

India (Bihar)

LGRani?

USSR (Vologda - Aristovo - ) UPTata

(scheme) (scheme)

China (Chinghai)

(scheme from Feistmantel, 1880)

(scheme from Nejburg, 1948)

(scheme from Mägdefrau, 1956)

(scheme from Feistmantel, 1880)

(scheme from Kon'no, 1960)

China (Guizhou/Yunnan)

India (Madhya Pradesh)

South Africa (Natal)

China (Guangxi)

China (Heilongjiang)

China (Sichuan)

China (Liaoning)

China (Guizhou/Yunnan)
(P--T)

UC-LP

(C-T)

(C-T)

(C-T)

(C-P)

(C-P)

UP

UP-LT

UPKaza-Tata

UP

UP

UP

UP

UP

UP 


\section{Schuetzia Geinitz 1863}

S. anomala Geinitz 1863

1979 Millay et Taylor, fig.2E

1979 Millay et Taylor, fig.15A

(rec. from Remy et Remy, 1959)

(scheme from Göppert, 1864 1865)

\section{Scirroma Chandra et Surange 1977}

S. sp.

1977 Chandra et Surange (d), fig. $1 \mathrm{~A}, \mathrm{C}$

S. angusta Chandra et Surange 1977

1977 Chandra et Surange (d), fig.1D; pl.1, 3-5 (cuti)

S. ventilebra Chandra et Surange 1977

1977 Chandra et Surange (d), fig.1B; pl.1, 6 (cuti)

India (West Bengal)

India (West Bengal)

India (West Bengal)
LGRani

LGRan

LGRani

\section{Scleromedulloxylon Doubinger et Marguerier 1975}

S. batoviense Guerra 1976

Remark: Guerra (1976) non vidi

1978 Guerra-Sommer, pl.1, 1-7; pl.2, 1-9; pl.3, 1-7

1980 Guerra-Sommer, pl.2, 1-5

S. varollense (Renault 1894) Marguerier 1975

1980 Marguerier et Pacaud, pl.4, 1, 2; pl.5, 1, 2

\section{Scolecopteris Zenker 1837}

S. arborescens (Sternberg 1825) Stur 1883

Remark: in text S. arborescens (Schlotheim) Stur 1976 Barthel et al., fig. 6

1980 Barthel (c), pl.5; pl.6; pl.8, 7, 8

1980 Barthel (c), pl.7

S. candolleana (Brongniart 1828) Stur 1883

1976 Barthel et al., pl.17, 1-4, 5-10 (spore), 11.

1980 Barthel (b), pl.1, A F

+ S. cyathea (Schlotheim 1804) Stur 1883

1980 Barthel (c), fig. p.278

1980 Barthel (c), pl.3, 1

1980 Barthel (c), pl.3, 2-9; pl.4; pl.8, 5, 6

Remark: pl.3, 1 is the specimen originally figured by Schlotheim (1804: pl.7)

S. densifolia (Göppert 1864) Barthel, Götzelt et Urban 1976

1976 Barthel et al., pl.15, 1, 2, 3 (spore)

1980 Barthel (b), pl.2, A C

G. hemitelioides (Brongniart 1833) Stur 1883

1976 Barthel et al., pl.14, 1, 2, 3-13 (spore)

1980 Barthel (b), pl.4, A

S. lepidorachis (Brongniart 1834) Barthel, Götzelt et Urban 1976

Remark: in text $S$. lepidorhachis

1976 Barthel (b), pl.2, 5

Barthel et al., pl.12, 1-12; pl.13, 1-8,

9. 24 (spore); pl.14, 14-21

S. oreopteridia (Sternberg 1825) Barthel 1980

Remark: in text S. oreopteridia (Schlotheim) Barthel 1980

1980 Barthel (c), pls.1, 2

S. permica (Němejc 1948) Barthel, Götzelt et Urban 1976

1976 Barthel et al., pl.15, 4-7, 8-10 (spore)

S. polymorpha (Brongniart 1828) Stur 1883

Brazil (Rio Grande do Sul)

$P$

Brazil (Rio Grande do Sul)

$\mathrm{P}$

France (Saône-et-Loire)

LPAutu

GDR (Karl-Marx-Stadt)

LPRotl

GDR (Gera)

GDR (Karl-Marx-Stadt)

LPRotl

LPRotl

GDR (Karl-Marx-Stadt) LPRotl

GDR (Gera)

LPRotl

(from Stur, 1883)

GDR (-)

LPRotl

GDR (Gera)

LPRotl

$\begin{array}{ll}\text { GDR (Karl-Marx-Stadt) } & \text { LPRotl } \\ \text { GDR (Gera) } & \text { LPRotl } \\ \text { GDR (Karl-Marx-Stadt) } & \text { LPRotl } \\ \text { GDR (Gera) } & \text { LPRotl }\end{array}$

GDR (Karl-Marx-Stadt) LPRotl

GDR (Karl-Marx-Stadt) ～LPRotl

GDR (Gera)

LPRotl

GDR (Karl-Marx-Stadt)

LPRotl

GDR (Gera)

LPRotl 
S. polypodioides (Presl 1838) Barthel, Götzelt et Urban 1976

1976 Barthel et al., fig.7A-C; pl.15, 11, 12-17

(spore); pl.16, 1-12

GDR (Karl-Marx-Stadt)

GDR (Gera)

LPRotl

1980 Barthel (b), pl.3

\section{Scopus Benecke 1976}

S. confertus Benecke 1976

1976 Benecke, fig.5

1976 Benecke, figs.46-54

Remark: organic connection and association

with Glossopteris sp.

S. didiscus Benecke 1976

1976 Benecke, fig.6

1976 Benecke, figs.69, 81-84, 93, 94

Remark: association with Glossopteris sp.

$$
\text { S. gibbosus Benecke } 1976
$$

1976 Benecke, fig.4

1976 Benecke, figs.42-45, 55-81, 85, 93, 94

Remark: association and (?) organic

connection with Glossopteris sp.

S: obscurus Benecke 1976

1976 Benecke, fig. 7

1976 Benecke, figs.86, 87, 89-94

Remark: organic connection with Glossopteris sp.

\section{Scutum Plumstead 1952}

S. sp.

1977 Pant, fig.8B

1977 Pant, fig.9A-C

1978 White, figs.59, 60

1978 White, figs. 61,62

Remark: organic connection with Glossopteris $\mathrm{sp}$.

S. dutoitides Plumstead 1953

1976 Schopf, fig.1("')

S. leslium Plumstead 1952

1979 Banerjee, pl.51, 15-19

Remark: organic connection with Glossopteris browniana

S. rubidgeum Plumstead 1953

1976 Schopf, fig.1("'")

S. sahnii Surange et Chandra 1974

1978 White, fig.58

Remark: organic connection with Glossopteris conspicua

1979 Chandra et Surange, pl.13, 2; pl.22, f.8

Remark: organic connection with Glossopteris maculata

Selaginella Palisot de Beauvois 1805 (nom. cons.)

S. harrisiana Townrow 1968

1980 Retallack, fig.21.5G

\section{Senftenbergia Corda 1845}

S. saxonica Barthel, Götzelt et Urban 1976

1976 Barthel (b), pl.1, 4 (spore); pl.2, 2, 2A (rec.)

South Africa (Natal)

UP

(rec.)

South Africa (Natal)

UP

(rec.)

South Africa (Natal)

UP

(rec.)

South Africa (Natal)

UP

(rec. from Surange et

Chandra, 1975)

(from Plumstead, 1958)

Australia (New South Wales) UP

Australia (New South Wales)

(scheme)

India (West Bengal)

UPRani

(scheme)

Australia (New South Wales)

P

India (Orissa)

LGRani

Australia (New South Wales)

L-UP

GDR (Karl-Marx-Stadt)

LPRotl 
1976 Barthel et al., pl.8, 1 5A, 6, 6A, B (spore);

pl.9, 1, 2, 3-7 (spore), 8, 9 (spore)

\section{Senotheca Banerjee 1969}

Remark: according to Farr et al. (1979, p.1602) possibly Senotheca Banerjee 1967

S. sp.

1976 Schopf, fig. $7 \mathrm{C}$

1979 Banerjee, pl.50, 9

S. murulidihensis Banerjee 1969

1976 Schopf, fig.1(".)'

1977 Pant, fig. 10B

1978 Rigby (a), figs.19, 20

1979 Banerjee, pl.50. 10

1979 Banerjee, pl.54. 8-10

Remark: organic connection with

$$
\text { Glossopteris clarkei }
$$

\section{Sigillaria Brongniart 1822}

S. sp.

1977 Feng et al., fig. 103

1977 Feng et al., pl.234, 14

1978 Gillespie et al., fig.7

1978 Ratkevich et La Fon, fig. p.49

1979 Yuasa, fig.5.4

1979 Yuasa, fig.5.5

1980 Tian et Zhang, pl.26, 2, 2A, 2B; pl.27. 1, 1A

1980 X. number " 1 " in fig.

S. brardii Brongniart 1828

1976 Barthel et al., pl.29, 12, 13

1978 Gillespie et al., pl.17, 1, 6

1978 Gillespie et al., pl.19, 3

S. ichthyolepis (Presl 1838) Corda 1845

1977 Feng et al., pl.233, 2

S. tessellata Brongniart 1828

1977 Feng et al., fig. 102

(from Banerjee, 1969)

India (West Bengal)

UPRani

(scheme)

(from Banerji, 1969)

Australia (New South Wales) UP

India (West Bengal)

UPRani

(rec.)

\begin{tabular}{|c|c|}
\hline (scheme) & $(\mathrm{C}-\mathrm{P})$ \\
\hline China (Hunan) & LP \\
\hline (rec.) & (UC $\cdot$ LP? \\
\hline USA (New Mexico) & LP \\
\hline (scheme from Hirmer, 1927) & $(\mathrm{C}-\mathrm{P})$ \\
\hline (scheme from Zeiller, 1914) & (C P) \\
\hline $\begin{array}{l}\text { China (Guizhou) } \\
\text { (rec. from Bertrand, 1943) }\end{array}$ & UP \\
\hline GDR (Karl-Marx-Stadt) & LPRotl \\
\hline USA (West Virginia) & UC LP? \\
\hline (scheme) & $(\mathrm{UC}-\mathrm{LP} ?$ \\
\hline China (Guangxi) & UP \\
\hline (scheme) & $(\mathrm{C} \cdot \mathrm{P})$ \\
\hline
\end{tabular}

Skokia Radchenko 1969

+ S. elongata Sukhov

1976 Durante, pl.72, 9, 10

Mongolia

L UP

Slivkovia Meien 1969

S. petschoriensis Meien 1969

1978 Meien, figs.20 23; pl.2, 9

USSR (Komi ASSR -- Pechora

$$
\text { Basin -...) }
$$

UP

Solenobrasilioxylon Mussa 1978

S. irinei Mussa 1978

1978 Mussa (b), pl.2, 610

Brazil (São Paulo)

$\mathrm{P}$

Sparsistomites Meien 1969

S. communis Glukhova 1980

1980 Glukhova et Men'shikova, fig.1; pl.13, 1-4 (cuti)

USSR (Kemerovskaya oblast .

Kuzbass …)

UP

Sphenobaiera Florin 1936

S. biloba Feng 1977

1977 Feng et al., pl.250, 1

China (Hunan)

UP 
S. digitata (Brongniart 1828) Florin 1936 1976 Daber et Helms, fig. p.75

1977 Krumbiegel et Walther, fig.2.103.7

1977 Krumbiegel et Walther, fig.2.108.1

1977 Remy et Remy, fig.39

1977 Schaumberg, fig.7

1980 Daber (b), figs.101, 102

1980 Daber (b), fig.103

S. macrofolia Burago 1977

1977 Burago, pl.25, 1, 3, 6; pl.26, 1, 4

S. spirata Sze 1974

1976 Huang, pl.224, 5-6(?)

S. tenuistriata (Halle 1927) Florin 1936

1978 Chen et al., pl.154, 3

$\begin{array}{ll}\text { GDR (Halle) } & \text { P } \\ \text { GDR (Halle) } & \text { UPKupf } \\ \text { (scheme from Mägdefrau) } & \\ \text { GDR (Halle) } & \text { UPKupf } \\ \text { GFR (Hessen) } & \begin{array}{l}\text { UPKupf } \\ \text { GDR (Halle) }\end{array} \\ \text { GDR (Erfurt) } & \text { LPRotl } \\ \text { USSR (Primorskiy Kray) } & \text { UP } \\ \text { China (Inner Mongolia) } & \text { L-UP } \\ \text { China (Sichuan) } & \text { UP }\end{array}$

Sphenopecopteris Zhang et Mo 1979

S. beaniata Zhang et Mo 1979

1979 Zhang et Mo, pl.1, 1-5; pl.2, 1

China (Henan)

LP

\section{Sphenophyllostachys Seward 1896}

Remark: genus name is Bowmanites Binney 1871. See Farr et al. (1979, p.1647)

S. sp.

1980 Babin, fig.8.15

(scheme from Hirmer)

(D-T)

\section{Sphenophyllum Brongniart 1828}

S. sp.

1976 Balasundaram et al., pl.22, 2

1976 Barthel (b), pl.3, 4

1976 Bohlin, figs.3, 4

1976 Bohlin, figs.313-315, 384, 386, 387, 464(?),

622-623; pl.19, 9, 10; pl.24, 7(?)

1976 Grzimek, fig.8.15

1976 Paliwal et al., fig.1

1977 Huang, pl.17, 5

1977 Zimina, pl.1, 7

1979 Major, fig.204

1979 Yuasa, fig.4.13

1980 Babin, fig.8.14A, B

1980 Zhao et al., pl.4, 6, 7

S. angustifolium (Germar 1845) Göppert 1848

1976 Barthel et al., pl.23, 1-10, 11-15 (spore)

1977 Remy et Remy, fig.244A

1977 Remy et Remy, fig.244B

1978 Gillespie et al., pl.29, 6, 7

1980 Storch, pl.12, 1

S. elagatiefolium Xi 1977

1977 Feng et al., pl.236, 1, 2

S. emarginatum (Brongniart 1822) Brongniart 1828

1976 Bohlin, figs.2, 5-7; pl.1, 5; pl.2, 10 (all cf.)

1978 Gillespie et al., pl.28, 2

1978 Gillespie et al., pl.28, 8; pl.29, 1

$S$. gongxianense Xi 1977

1977 Feng et al., pl.235, 11

S. kawasakii Stockmans et Mathieu 1957

1976 Huang, pl.207, 3; pl.209, 5

S. koboense Kobatake 1957

1977 Feng et al., pl.236, 9

1980 Huang, pl.217, 1-4; pl.218, 4

$\begin{array}{ll}\text { (scheme) } & \\ \text { GDR (Karl-Marx-Stadt) } & \text { LPRotl } \\ \text { China (Kansu) } & \text { UC-LP } \\ \text { China (Chinghai) } & \text { UC-LP } \\ \text { (rec.) } & \\ \text { (scheme) } & \\ \text { China (Heilongjiang) } & \text { UP } \\ \text { USSR (Primorskiy Kray) } & \text { LP } \\ \text { (scheme) } & \text { (D-T) } \\ \text { (scheme from Hirmer, 1927) } & \text { (C-P) } \\ \text { (scheme) } & \text { (D-T) } \\ \text { China (Guizhou/Yunnan) } & \text { UP } \\ & \\ \text { GDR (Karl-Marx-Stadt) } & \text { LPRotl } \\ \text { GDR (Gera) } & \text { LPAutu } \\ \text { (scheme) } & \text { (C-P) } \\ \text { USA (West Virginia) } & \text { UC-LP? } \\ \text { GDR (Gera) } & \text { LP } \\ \text { China (Henan) } & \\ \text { China (Kansu) } & \text { LP } \\ \text { (scheme) } & \\ \text { USA (West Virginia) } & \text { UC-LP } \\ \text { China (Henan) } & \text { UC-LP? } \\ \text { China (Inner Mongolia) } & \text { LP } \\ \text { China (Henan) } & \\ \text { China (Liaoning) } & \text { UC-LP } \\ & \\ & \text { UP } \\ \text { UP } & \end{array}$


1980 Huang, pl.217, 5 $6 ;$ pl.218, 56

1980 Tian et Zhang, pl.4, 3 5, 7. 7A; pl.23, 1

1980 Zhao et al., pl.3, 5

S. majus (Bronn 1828) Bronn 1835

1978 Gillespie et al., pl.29, 3

S. meyenii Zimina 1977

1977 Zimina, pl.1, 13

S. minor (Sterzel 1895) Gu et Zhi 1974

1976 Huang, pl.210, 6, 7

S. oblongifolium (Germar et Kaulfuss 1831) Germar 1845

1976 Barthel (b), pl.1, 1; pl.2, 3, 4

1976 Barthel et al., fig.8A C; pl.19, 1-4A, 5 15: pl.20, $1 \cdots$;

pl.21. 1 9; pl.22, 1 3; pl.24, 1 5; pl.30, 9, 10

Remark: descr./ills. include cuticles and spores

1976 Huang, pl.208, 2.4

1977 Krumbiegel et Walther, fig.2.92.8 9

1977 Remy et Remy, fig. $246 \mathrm{C}$

1978 Gillespie et al.. pl.29, 4, 5

1980 Huang, pl.217, 7

1980 Huang, pl.217, 89

1980 Storch et Barthel, pl.3, 1.8

1977 Zimina, fig. $9 ;$ pl.1, 46

S. sakoense Appert 1977

1977 Appert, pl.7, 2 5; pl.8

S. scopulatum Sze 1974

1976 Huang, pl.210, 13

S. sino-coreanum Yabe 1920

1978 Zhang, pl.150, 10

S. sino-coreanum Yabe $1920 \mathrm{ssp}$. dentatum Zhang 1978

1978 Zhang, pl.151, 7

1980 Zhao et al., pl.6. 1, 2

S. spathulatum Sze 1974

1976 Huang, pl.210, 4, 5

S. speciosum (Royle 1833) McClelland 1850

1976 Hsü, pl.1, 1A, 2. 3

1978 Rösler. pl.1, 7 (cf.)

1978 Zhang, pl.150, 3

1979 Van Dijk et al.. pl.45, 7-12

1980 Huang, pl.217. 11 (cf.)

S. thonii Mahr 1868

1976 Barthel (b), pl.4, 7

1976 Barthel et al, pl.24, 8, 9

1976 Le Roux, pl.2, 810 (cf.)

1977 Feng et al., pl.235, 12

1977 Krumbiegel et Walther, fig.2.92.10

1977 Remy et Remy, fig.247A, B

1977 Remy et Remy, fig.247C

1980 Huang, pl.237. 6

$S$. verticillatum Zeiller 1885

1977 Feng et al., pl.235, 6

S. yiyangense $\mathrm{Xi} 197 \%$

1977 Feng et al., pl.236, 36

$S$ yujicense Huang 1980

1980 Huang, pl.218. 1 ?
China (Jilin)

UP

China (Guizhou)

UP

China (Guizhou/Yunnan)

USA (West Virginia)

$\mathrm{UC} \mathrm{LP}$ ?

USSR (Primorskiy Kray)

LP

China (Inner Mongolia)

L. UP

GDR (Karl-Marx-Stadt)

LPRotl

GDR (Karl-Marx-Stadt)

LPRotl

China (Inner Mongolia)

(scheme)

(scheme)

USA (West Virginia)

China (Liaoning)

China (Jilin)

GDR (Karl-Marx-Stadt)

USSR (Primorskiy Kray)

Madagascar (Sakoa Basin)

China (Inner Mongolia)

China (Guizhou)

China (Guizhou)

China (Guizhou/Yunnan)

China (Inner Mongolia)

China (Xizang)

Brazil (Paraná)

China (Guizhou)

South Africa (Natal)

China (Jilin)

GDR (Karl-Marx-Stadt)

GDR (Karl-Marx-Stadt)

South Africa (Transvaal)

China (Henan)

(scheme)

GDR (Gera)

(scheme)

China (Jilin)

China (Henan)

('hina (Henan)

China (Liaoning)

China (Inner Mongolia)

$\mathrm{UC} \cdots \mathrm{LP}$

(C $\mathrm{P})$

UC LP?

UC LP

UC LP

[SPRot]

LP

LP

UP

UP

UP

L.UP

UP

UP

UP

UP

UP

LPRotl

LPRotl

LP

LP

LPAutu

$L P$

L.P

LP

LP
S. osipoviense Zimina 1977

LGKarh Bara?

\section{Sphenopteridium Schimper 1874}

S. pseudogermanicum (Halle 1927) Gu et Zhi 1974

1976 Huang, pl.213. 13 
1977 Feng et al., pl.241, 5

1980 Tian et Zhang, pl.7, 3, 3A

\section{Sphenopteris (Brongniart 1822) Sternberg 1825}

$S$. sp.

1976 Balasundaram et al., pl.22, 4

1976 Bohlin, figs.138bis, 197, 198, 203(?), 206bis(?), 534 (cf.),

pl.12, 6(?), 8, 9; pl.13, 1, 3; pl.31, 6 (cf.)

1976 Bohlin, figs. $202,445,450(?), 452,454,461(?)$,

462-463, 485, 677(?), 690; pl.20, 7; pl.21, 4-5 ("');

pl.22, 13 (cf.); pl.23, 6(?); pl.26, 6, 7; pl.28, 12(?)

1976 Huang, pl.214, 2

1977 Appert, pl.9, 6-12

1977 Bose et al., fig.2B; pl.1, 9 (all ?)

1979 Van Dijk et al., pl.45, 10, 16(?)

1979 Yuasa, fig. 3.6

1980 Tian et Zhang, pl.7, 1, 1A

S. alata (Brongniart 1829) Presl 1838

1978 Cazzulo-Klepzig, fig.6A; pl.2, 1-2

Remark: in text fig.6; pl.10, 1-2

1979 Van Dijk et al., pl.46, 15

S. damesi (Stur 1883) Zeiller 1907

1976 Bohlin, fig.205 (cf.)

S. dichotoma Althaus 1846

1977 Schaumberg, fig.4

S. firmata Sze 1974

1976 Huang, pl.212, 2, 2A; pl.215, 1

$S$. fossorum Zeiller 1888

1977 Tenchov (a), pl.2, 1

S. germanica Weiss 1879

1976 Alvarez-Ramis et Doubinger, fig.5

1976 Barthel et al., pl.31, 4

1977 Remy et Remy, fig.87A-E

1978 Remy, fig.1

1980 Pfefferkorn et Resnik, pl.1, 1-4

S. gongxianensis Xi 1977

1977 Feng et al., pl.241, 1, 2

S. grabaui Halle 1927

1976 Durante, pl.23, 3 (aff.), 4-5 (ex gr.)

$S$. guetzoldii Gutbier 1849

1976 Barthel et al., pl.10, 9, 10

S. heilongjiangensis Huang 1977

1977 Huang, fig.6; pl.9, 5; pl.10, 1; pl.11, 1-7

S. hughesii (Feistmantel 1881) Arber 1905

1976 Lele (b), fig.6

S. huihuipuensis Bohlin 1976

1976 Bohlin, figs.200, 201, 206 (cf.); pl.12, 7; pl.13, 2, 4

S. incrassata Nejburg 1948

1977 Huang, pl.7, 4; pl.8, 2-3

S. interrupte-pinnata Kutorga 1838

1978 Remy et Remy (c), fig.1

1978 Remy et Remy, pl.7, 9 (cf., "’)

S. kukukiana Gothan et Nagalhard 1922

1977 Remy et Remy, fig.89A, B

1978 Remy et Remy (c), pl.7, 4--5,6 (?, cf.)

S. lobifolia Morris 1845

1978 Cazzulo-Klepzig, fig.6B; p1.3, 3

Remark: in text fig.6; pl.7, 2

S. mathetii Zeiller 1888

1976 Barthel (b), pl.1, 7

1976 Barthel et al., pl.40, 1-7
China (Guangdong)

UP

China (Guizhou)

UP

(scheme)

China (Kansu)

UC-LP

China (Chinghai)

$\mathrm{UC}-\mathrm{LP}$

China (Inner Mongolia)

Madagascar (Sakoa Basin)

India (Madhya Pradesh)

South Africa (Natal)

(scheme from Halle, 1931)

China (Guizhou)

UP

LGKarh-Bara?

UP-LT

UP

(C-P)

$\mathrm{UP}$

Brazil (Santa Catarina)

UPKaza-Tata

South Africa (Natal) UP

China (Kansu)

UC-LP

GFR (Hessen)

UPKupf

China (Inner Mongolia)

L-UP

Bulgaria (Northwest)

LP

France (Loire)

GDR (Leipzig)

GDR (Suhl)

(rec.)

USA (Colorado)

China (Henan)

Mongolia

GDR (Karl-Marx-Stadt)

China (Heilongjiang)

(scheme)

China (Kansu)

China (Heilongjiang)

USSR (West Ural)

Italy (Brescia)

Italy (Brescia)

Italy (Brescia)

Brazil (Santa Catarina)

GDR (Karl-Marx-Stadt)

GDR (Karl-Marx-Stadt)
UP

LPRotl

UP

UC-LP

LP

UPKupf

LPSaxo

LPSaxo

LPSaxo

UPKaza-Tata

UCStep-LPAutu

LPRotl

LPAutu

$\mathbf{P}$

$P$

LPRotl

LPRotl 
S. patens (Althaus 1846) Geinitz 1848

1977 Schaumberg, fig. 5

1978 Remy et Remy (c), pl.7, 7, 8

S. polymorpha Feistmantel 1876

1976 Lele (b), fig. $7 \mathrm{~A}$

S. rotundata Sze 1974

Remark: in text $S$. rotunda

1976 Huang, pl.212, 3, 3A

S. sellardsii Baxter 1978

1978 Baxter, figs. 14

S. suessii Geinitz 1869

1977 Remy et Remy, fig.88

1978 Remy et Remy (c), pl.7, 1 3

S. taiyuanensis Halle 1927

1976 Durante, fig.30: pl.20, 4, 5; pl.46, 4

S. tenuis Schenk 1883

1977 Feng et al., pl.240, 1

1978 Zhang, pl.153, 3

1979 Asama, fig. 17D

S. tunguscana (Schmalhausen 1879) Zalessky 1918

1976 Durante, pl.18, 1 4; pl.23, 1

S. yichunensis Huang 1977

1977 Huang, pl.8, 1

$\begin{array}{ll}\begin{array}{l}\text { GFR (Hessen) } \\ \text { Italy (Brescia) }\end{array} & \begin{array}{l}\text { UPKupf } \\ \text { LPSaxo }\end{array} \\ \text { (scheme) } & \\ \text { China (Inner Mongolia) } & \text { L-UP } \\ \text { USA (Kansas) } & \text { LP } \\ \text { Italy (Brescia) } & \text { LPSaxo } \\ \text { Italy (Brescia) } & \text { LPSaxo } \\ \text { Mongolia } & \text { UP } \\ \text { China (Henan) } & \\ \text { China (Guizhou) } & \text { LP } \\ \text { China (Shanxi) } & \text { UP } \\ \text { Mongolia } & \text { P } \\ \text { China (Heilongjiang) } & \text { LP }\end{array}$

Sphenozamites (Brongniart 1849) Miquel 1851

S. sp.

1979 Vozenin-Serra, pl.2, 9; pl.3, 10-14 (cuti) (all ?)

S. permicus Burago 1977

1977 Burago, pl.27, 1. 2

\section{Sphyropteris Stur 1883}

S. sp.

1977 Remy et Remy, fig.59

\section{Sporophyllites Chalyshev ex Fefilova 1978}

S. petschorensis (Schmalhausen 1879) Fefilova 1978 1978 Fefilova (a), figs.1 7; pl.1, 1 5; pl.2, f.1 5

Remark: descr./ills. include cuticles and spores (Vitreisporites)

\section{Squamella White 1978}

S. ampla White 1978

1978 White, figs.27. 31

1978 White, fig. 32

Remark: association with Glossopteris ampla

S. australis White 1978

1978 White, fig.1

1978 White, figs.3 25

1978 White, fig. 35

Remark: organic connection with Glossopteris australis and sporangia.

The species includes "Lidgettonia" australis White 1964

S. ovulifera White 1978

1978 White, fig.26

Remark: organic connection with leaf fragment and seed
Australia (New South Wales) (scheme)

(scheme)

Australia (New South Wales) (rec.)

Australia (New South Wales) 


\section{Stellotheca Surange et Prakash 1962}

Remark: non Stellotheca Danzê-Corsin 1956. Substitute name Lelstotheca Maheshwari 1972. See Farr et al. (1979, p.951)

S. robusta (Feistmantel 1880) Surange et Prakash 1962 1978 Maithy et Mandal, figs.1-3; pl.1, 1-3; pl.2, 5-9

\section{Stephanophyllites Millan et Dolianiti 1977}

Remark: mentioned as new genus by Millan et Dolianiti (1979, p.1). 1977 paper unknown to the compilers of IFPM

S. sanpaulensis Millan et Dolianiti 1977 1979 Millan et Dolianiti, fig.2

1980 Millan et Dolianiti, fig.1; pl.1, 1-4;

pl.2, 5-11; pl.3, 12-17

1980 Millan et Dolianiti, fig.2

\section{+ Stephanostoma Pant et Nautiyal 1960}

Remark: non Stephanostoma (Mitten 1873) Kindberg 1899 See Farr et al. (1979, p.1677)

S. crystallinus Pant et Nautiyal 1960

1977 Pant, fig.6A

(from Pant et Nautiyal, 1960

\section{Stigmaria Brongniart 1822}

S. sp.

1980 Tian et Zhang, pl.24, 2-4A; pl.25, 4

S. ficoides (Sternberg 1820) Brongniart 1822

1978 Chen et al., pl.150, 1

1978 Gillespie et al., pl.19, 1; pl.21, 3-5

1978 Tian et Zhang, pl.3, 3

1978 Zhang, pl.150, 1

1978 Zhao et al., pl.2, 9

S. rugulosa Gothan 1923

1977 Feng et al., pl.234, 5

1978 Zhang, pl.150, 2

1980 Tian et Zhang, pl.4, 1, 2

$\begin{array}{ll}\text { China (Guizhou) } & \text { UP } \\ \text { China (Sichuan) } & \text { UP } \\ \text { USA (West Virginia) } & \text { C-LP? } \\ \text { China (Guizhou) } & \text { UP } \\ \text { China (Guizhou) } & \text { UP } \\ \text { China (Guizhou/Yunnan) } & \text { UP } \\ & \\ \text { China (Guangdong) } & \text { UP } \\ \text { China (Guizhou) } & \text { UP } \\ \text { China (Guizhou) } & \text { UP }\end{array}$

\section{Stockmansia Bohlin 1979}

S. salviniaefolia (Stockmans et Mathieu 1939) Bohlin 1976 1976 Bohlin, figs.178, 179, 687, 705, 727; pl.9,

$3-9 ; \mathrm{pl} .31,3$; pl.32, 10-12

China (Kansu)

UC-LP

\section{Strigillotheca Gu et Zhi 1974}

S. fasciculata $\mathrm{Gu}$ et $\mathrm{Zhi} 1974$ 1978 Zhang, pl.164, 8, 9

1980 Zhao et al., pl.22, 4
China (Guizhou)

China (Guizhou/Yunnan)
UP

UP

\section{Stylocalamites Weiss 1884}

S. sp.

1978 Gillespie et al., fig.10F (scheme)

(C-P) 


\section{Subsigillaria Mercenier 1913}

S. sp

1977 Krumbiegel et Walther, fig.2.89.2

(scheme from Potonie)

\section{Supaia White 1929}

S. linearifolia White 1929

1976 Doubinger et Heyler, fig.1

1980 Huang, fig.34

S. serrata $\mathrm{Hu} 1980$

$1980 \mathrm{Hu}$, fig.1; pl.1, 4 (all ?)

S. shenshuensis Huang 1977

1977 Huang, fig. 15; pl.27, 1; pl.40. 1

S. thinnfeldioides White 1929

1976 Doubinger et Heyler, pl.1, 13 (aff.)

S. tieliensis Huang 1977

1977 Huang, fig. 14 ; pl.36, 1 3; pl.37, 1 2; pl.38, 1 - 5

1980 Huang, pl.253, 1 ; pl.261, 12

Remark: in text $S$. teiliensis

(rec. from White, 1929)

(rec.)

China (Xinjiang)

UP

China (Heilongjiang)

UP

France (Hérault)

LPAutu?

China (Heilongjiang)

China (Heilongjiang)

UP

UP

China (Kansu)

China (Xizang)

UC LP

UP

(from Zalessky)

1976 Bohlin, fig. 694

Syniopteris Zalessky 1929

$S$. sp.

1980 Durante, fig. 4

China (Xizang)

UP

\section{Syrjagia Fefilova 1973}

S. lituata Fefilova 1973

1978 Fefilova (b), fig.15; pls.14, 15

S. rectiserialis Fefilova 1973

1978 Fefilova (b), fig.16; pl.16

USSR (Komi ASSR)

UPKaza

USSR (Komi ASSR)

LPUfim

\section{Taeniopteris Brongniart 1828}

T. sp.

1976 Bohlin, fig.184 (cf.)

1976 Bohlin, fig.300; pl.17, 9, 10

1976 Durante, pl.57, 1, 6

1978 Burago, fig. $3 ;$ pl.9, 7, 8, 10, 11

1979 He et al., pl.56, 1

1980 Tian et Zhang. pl.12, 3; pl.17, 2; pl.18, 1

T. abnormis Gutbier 1835

Remark: according to Barthel correct

name for specimens commonly identified

as T. multinervis

1976 Barthel (b), pl.4, 11

Barthel et al., pl.38, 1\%5

T. crassinervis Mo 1980

1980 Zhao et al., pl.18, 6, 7

$T$. densissima Halle 1927

1980 Huang, pl.226, 8: pl.240, 7 (all cf.)
China (Kansu)

China (Chinghai)

Mongolia

USSR (Primorskiy Kray)

China (Guizhou)

China (Guizhou)

GDR (Karl-Marx-Stadt)

GDR (Karl-Marx-Stadt)

LPRotl

LPRotl

China (Guizhou/Yunnan)

UP

China (Liaoning)

UP
UC LP

UC-LP

UP

UP

UP

UP 
T. doubingeri Remy et Remy 1975

1977 Remy et Remy, fig.67A, B

T. eckardtii Germar MS ex Kurtze 1839

1977 Krumbiegel et Walther, fig.2.103.6

1977 Schaumberg, fig.6

T. guangxingensis Huang 1980

1980 Huang, pl.240, 4

T. hunanensis $\mathrm{Gu}$ et $\mathrm{Zhi} 1974$

1977 Feng et al., pl.249, 1, 2

T. integra Stockmans et Mathieu 1957

1980 Huang, fig.41; pl.240, 1

$T$. jejunata Brongniart 1828

1976 Barthel et al., pl.38, 6, 7, 8-9 (cuti)

1977 Krumbiegel et Walther, fig.2.96.10

1977 Remy et Remy, fig.65A

1977 Remy et Remy, fig.65C

T. multinervis Weiss 1869

1977 Remy et Remy, fig.66A, B

Remark: in text T. multinervia

1978 Chen et al., pl.153, 4

T. multinervis Weiss 1869 fa. fallax Göppert 1864-1865

1979 Doubinger et Alvarez-Ramis, pl.1, 1; pl.2, 1, 3

T. norinii Halle 1927

1976 Huang, pl.221, 6

1978 Chen et al., pl.153, 5

T. nystroemii Halle 1927

1978 Zhang, pl.161, 3 (cf.)

1980 Tian et Zhang, pl.19, 7

T. primorjensis Burago 1978

1978 Burago, pl.9, 1-6

T. punctifera Appert 1977

1977 Appert, pl.34, 4-9; pl.35, 1-6

T. rarinervis Zhao 1980

1980 Zhao et al., pl.22, 1-1A(?)

T. sinegorkiensis Burago 1978

1978 Burago, pl.9, 9

$+T$. szei Chow

1980 Huang, fig.40; pl.222, 3-7; pl.240, 5

T. taiyuanensis Halle 1927

1977 Feng et al., pl.249, 3

1978 Chen et al., pl.155, 5

1980 Huang, pl.240, 2-3A (cf.)

T. tingii Halle 1927

1976 Huang, pl.211, 5

1977 Feng et al., pl.249, 4

\begin{tabular}{|c|c|}
\hline France (Saône-et-Loire) & LPAutu \\
\hline GDR (Halle) & UPKupf \\
\hline GFR (Hessen) & UPKupf \\
\hline China (Liaoning) & UP \\
\hline China (Henan) & UP \\
\hline China (Liaoning) & LP \\
\hline $\begin{array}{l}\text { GDR (Karl-Marx-Stadt) } \\
\text { (scheme) }\end{array}$ & LPRotl \\
\hline $\begin{array}{l}\text { GDR (Gera) } \\
\text { (from Remy, 1953) }\end{array}$ & $\begin{array}{l}\text { LPAutu } \\
\text { (C-P) }\end{array}$ \\
\hline GFR (Saarland) & LPAutu \\
\hline China (Sichuan) & UP \\
\hline France (Vosges) & LPAutu \\
\hline China (Inner Mongolia) & LP \\
\hline China (Sichuan) & UP \\
\hline China (Guizhou) & UP \\
\hline China (Guizhou) & UP \\
\hline USSR (Primorskiy Kray) & UP \\
\hline Madagascar (Sakoa Basin) & LGKarh-Bara? \\
\hline China (Guizhou/Yunnan) & UP \\
\hline USSR (Primorskiy Kray) & UP \\
\hline China (Liaoning) & L-UP \\
\hline China (Henan) & UP \\
\hline China (Sichuan) & UP \\
\hline China (Jilin) & LP \\
\hline China (Inner Mongolia) & LP \\
\hline China (Henan) & UP \\
\hline
\end{tabular}

\section{Tatarina Meien 1969}

T. conspicua Goman'kov et Meien 1979

1979 Goman'kov et Meien, figs.1, 2G,D, 6-9 (cuti);

pl.14, 7; pl.15, 1-2 (cuti)

T. lobata Meien 1980

1980 Meien et Goman'kov, figs.8, 9-10 (cuti); pl.13, 1, 2, 3-5 (cuti) USSR (Krasnoyarskiy Kray

T. pinnata Goman'kov et Meien 1979

1979 Goman'kov et Meien, fig.2A, V, B; pl.15, 3 (cuti)

T. sadounikovii Meien 1980

1980 Meien et Goman'kov, figs.11-13; pl.13, 6-10

$$
\text { - Tungusk Basin -) }
$$

UP

USSR (Vologda - Aristovo --) UPTata

USSR (Krasnoyarskiy Kray

- Tungusk Basin -)

Remark: descr./ills. include cuticles 
T. sinuosa Goman'kov 1980

1980 Meien et Goman'kov, figs. $-5 ;$ pl.12, 1-7

USSR (Vologda -... Aristovo - ?)

UP

Remark: descr./ills. include cuticles

T. verrucosa Goman'kov 1980

1980 Meien et Goman'kov, figs.6-7 (cuti); pl.12, 8-9 (cuti)

USSR (Vologda -- Aristovo - ?) UP

\section{Taxopitys Kräusel 1928}

T. africana Kräusel 1928

1978 Mussa (a), fig.2C

T. indica Prasad et Chandra 1978

1978 Prasad et Chandra (a), figs. 1.4

T. jollyi Kräusel 1928

1978 Mussa (a), fig.3A, B; pl.1, 1 3

(scheme from Kräusel)

India (Maharashtra)

UPKamt

\section{Tchernovia Zalessky 1930}

Remark: commonly spelled Tschernovia. See Farr et al. (1979, p.1727)

T. sp.

1976 Durante, pl.56, 5; pl.57, 7

Mongolia

\section{Telangium Benson 1904}

$T . \mathrm{sp}$.

1978 Remy et Remy (c), pl.8, 12-14 (aff.)

Italy (Brescia)

LPSaxo

\section{Thallites Kidston ex Walton 1925}

T. sp.

1980 Zhao et al., pl.1, 4

China (Guizhou/Yunnan)

\section{Thamnopteris Brongniart 1849}

T. sp.

1976 Fischer et Gayrard-Valy, fig.81

Australia (.)

$\mathrm{P}$

T. schlechtendalii (Eichwald 1842) Brongniart 1849

1980 Bold et al., fig.19-66

(from Delevoryas)

\section{Thinnfeldia Ettingshausen 1852}

T. callipteroides Carpentier 1935

1976 Gould, fig. $4 \mathrm{H}\left({ }^{\prime \prime \prime \prime}\right)$

1977 Retallack, fig.6(1-3)(")')

1980 Retallack, fig.21.6B-C("'), fig.21.6D("'", cuti)

(scheme)

(P-T)

Australia (New South Wales)

UP

UP

\section{Tingia Halle 1925}

T. sp.

1976 Bohlin, fig.627(?)

T. carbonica (Schenk 1883) Halle 1925

1976 Asama, fig.1(14)

1977 De la Peña et al., pl.3, 4

1977 Feng et al., pl.239, 8

1979 Asama, fig.3(14)

1980 Asama, fig.5

1980 Huang, pl.226, $1-5$

T. crassinervis Halle 1925

1976 Asama, fig.1(15)

1979 Asama, fig.3(15)

1980 Asama, fig. 5

T. hamaguchii Kon'no 1929

1976 Huang, pl.211, $1-3$

1980 Huang, pl.226, 7

China (Chinghai)

UC-LP

(scheme)

Spain (Soria)

China (Guangdong)

(scheme)

(scheme)

China (Liaoning)

(scheme)

(scheme)

(scheme)

China (Inner Mongolia)

China (Liaoning)
LPAutu

UP

(C-P)

LP

LP 
T. partita Halle 1927

1976 Asama, fig.1(13)

1979 Asama, fig.3(13)

1980 Asama, fig. 5

1980 Huang, pl.226, 6; pl.228, 1

T. yichuanensis Feng 1977

1977 Feng et al., pl.238, 9

\section{Tingiostachya Kon'no 1929}

T. sp.

1977 Feng et al., pl.240, 9

\section{Tordoxylon Kräusel 1956}

T. sanpaulense Mussa 1978

1978 Mussa (c), figs.2A, B, 4; pl.1, 1-5; pl.2, 6-10;

pl.3, 11-17; pl.6, 38

1978 Mussa, fig.3A

T. steynspruitense Kräusel 1956 .

1978 Mussa (c), fig.2C

Remark: in text $T$. steinprutense

\section{Trichopitys Saporta 1875}

T. gracilis (Boersma et Visscher 1969) Remy et Remy 1977 1977 Remy et Remy, fig.38

T. heteromorpha Saporta 1875

1976 Bohlin, fig.692

Remark: in text $T$. heterophylla

1977 Remy et Remy, fig.37A

1977 Remy et Remy, fig.37B

\section{Tricoemplectopteris Asama 1959}

T. taiyuanensis Asama 1959

1976 Asama, fig.1(4)

1976 Asama, pl.29, f.1

1979 Asama, fig.2A

1979 Asama, figs.3(4), 4A

1980 Asama, fig.5

\section{Trigonocarpum Brongniart 1828 ex Endlicher 1837}

Remark: commonly spelled Trigonocarpus.

$$
\text { See Farr et al. (1979, p.1795) }
$$

$T$. sp.

1976 Barthel et al., pl.39, 2

1978 Gillespie et al., pl.37, 3, 4, 6, 7, 9

T. laguncula Bohlin 1976

1976 Bohlin, figs.229, 232, 234, 235(?), 238-241,

$243,275,521,523 ;$ pl.15, 7, 10-12; pl.16, 1 .

13 (cf.); pl.27, 14-18

$T$. noeggerathii Brongniart 1828

1976 Barthel et al., pl.40, 9

\section{Trithecopteris Pant et Misra 1977}

$T$. gondwanensis Pant et Misra 1977

1977 Pant et Misra, figs.3, 4; pl.3, 1-6; pl.4, 1-11

Remark: descr./ills. include cuticles and spores
China (Henan)

LP

(scheme)

(scheme)

(scheme)

China (Liaoning) LP

China (Henan) UP

Brazil (São Paulo) M-UP

(rec.)

South Africa (Orange Free State) Ecca

France (Var)

LPSaxo-UP

(from Florin, 1949)

GDR (Erfurt)

LPAutu

France (Hérault)

LPAutu

(scheme)

China (Shanxi) UP

China (Shanxi) UP

(scheme)

(scheme)

GDR (Karl-Marx-Stadt)

LPRotl

USA (West Virginia)

UC-LP?

China (Kansu)

UC-LP

GDR (Karl-Marx-Stadt)

LPRotl

India (West Bengal)

UPRani 


\section{Trizygia Royle 1834}

+T. grandifolia (Kobatake 1957)

1976 Asama, fig.1(18)

1979 Asama, fig.3(18)

1979 Yuasa, fig.4.14

$+T$. oblongifolia (Germar et Kaulfuss 1831)

1976 Asama, fig.1(16)

1979 Asama, fig. $3(16)$

T. sino-coreana (Yabe 1922) Asama 1970

1976 Asama, fig.1(18)

1979 Asama, fig.3(18)

$T$. speciosa Royle 1834

1976 Asama, fg.1(17)

1978 Maithy, figs.1 4; pl.1, 1 6; pl.2, 79

1978 Maithy, fig.5

Remark: descr./ills. include cuticles and anatomical details

1979 Asama, fig.3(17)

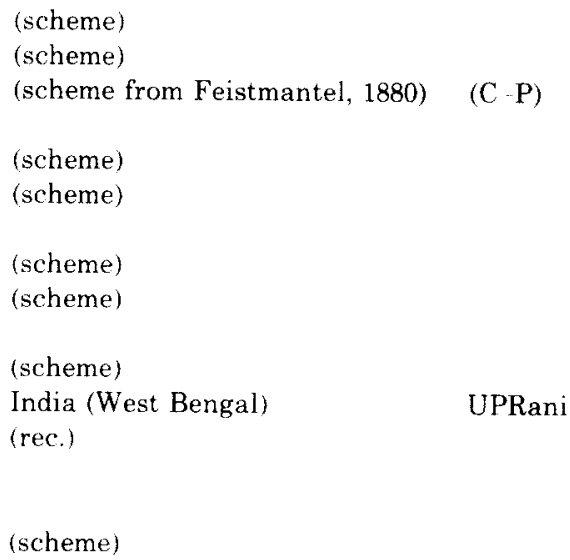

\section{Tubicaulis Cotta 1832}

T. solenites (Sprengel 1828) Cotta 1832

1976 Barthel et al., pl.2. 2

GDR (Karl-Marx-Stadt)

LPRot1

\section{Tungussocarpus Sukhov 1969}

T. sp.

1980 Goman'kov et Meien, fig.1(6)

T. tychtensis (Zalessky 1937) Sukhov 1969

1976 Durante, pl.72, 28

(scheme)

Mongolia

UP

\section{Tychtopteris Zalessky 1930}

$T$. arunensis Huang 1980

1980 Huang, fig. 36 ; pl.258, 5

T. cuneata Zalessky 1930

1976 Durante, pl.55. 1, 2

1977 Huang, pl.22, 4

$T$. densinervis $\mathrm{Hu} 1980$

$1980 \mathrm{Hu}, \mathrm{pl} .2,46$

T. obesa Hu 1980

$1980 \mathrm{Hu}$, pl.2, $13 \mathrm{~A}$

China (Heilongjiang)

Mongolia

UP

China (Heilongjiang)

China (Xinjiang)

China (Xinjiang)

\section{Tylodendron Weiss 1870}

T. speciosum Weiss 1870

1980 Guerra-Sommer, pl.1, 1-3

1980 Guerra-Sommer, pl.1, 4, 5

Remark: in text T. speciosum (Weiss) Florin 1940.

Brazil (Paraná)

UC LP See Farr et al. (1979, p.1818)

\section{Ullmannia Göppert 1850}

$U . \mathrm{sp}$.

1977 Jung, fig.2

1979 Asama, fig.12I

1979 Yuasa, fig.5.9

Remark: in text Ulmannia

U. bronnii Göppert 1850

1977 Feng et al., pl.251, 34

1977 Feng et al., pl.251. 5

1977 Jung, pl.17, 3

Italy (Bolzano)

(scheme)

(scheme from Florin. 1944)

China (Guangxi)

UP

China (Guangdong)

UP

Italy (Bolzano) 
1977 Krumbiegel et Walther, fig.2.103.1

1977 Remy et Remy, fig.46C

1977 Schaumberg, fig.11

1977 Vogellehner (b), fig.3C, D

1978 Miller, fig.2D

1978 Zhang, pl.165, 14

1980 Kämpfe, fig. $7.13 \mathrm{~F}$

1980 Zhao et al., pl.17, 6-6A (cf.)

$+U$. frumentaria (Schlotheim 1820) Göppert 1850

1977 Feng et al., pl.251, 1, 2

1977 Jung, pl.18, 4 (cf.)

1977 Krumbiegel et Walther, fig.2.102

1977 Remy et Remy, fig.46A

1977 Remy et Remy, fig.46B

1977 Schaumberg, figs. $8-10$

1978 Daber et Helms, fig. p.196

1978 Doubinger et al., pl.1, 3-5; pl.2, 7-9

1978 Remy et Remy (c), pl.8, 17, 18, 19-20 (cf.)

1980 Zhao et al., pl.6, 5-6 (cf.)

U. lycopodioides (Brongniart 1828) Göppert 1850

1979 Schniepp, fig. 14

\section{Umbellaphyllites Rasskazova 1961}

Remark: in text Umbelliphyllites

$U$. annularioides Rasskazova 1961

1977 De la Peña et al., pl.3, 5, 6

$U$. nacumilensis Verbitskaya 1980

1980 Verbitskaya (c), pl.8, 2-5

\section{Urnatopteris Kidston 1884}

$U$. sp.

1977 Remy et Remy, fig.60

\section{Uskatia Nejburg 1960}

U. conferta Nejburg 1960

1979 Lacey, fig.1(5)

U. dentata Feflova 1978

1978 Fefilova (b), fig.9A, G; pl.9, 1-2

1978 Fefilova (b), fig.9B, V, D; pl.9, 3

U. vicina Fefilova 1978

1978 Fefilova (b), fig.10; pl.8, 1, 2, 3 (cf.)

\section{Validopteris Bertrand 1932}

$V$. sp.

1976 Bohlin, fig.287("')

1976 Bohlin, fig.715; pl.30, 2("')

$V$. kokonoriensis Bohlin 1976

1976 Bohlin, figs.280, 576, 680; pl.17, 8; pl.18, 1-2 (all "'”)

\section{Vannus Plumstead 1963}

Remark: in text Vannus Plumstead 1962. See Farr et al. (1979, p.1837)

V. sp.

1978 White, figs, 67,68

Remark: organic connection with sporangial clusters

$\begin{array}{ll}\begin{array}{l}\text { GFR (Rheinland-Westfalen) } \\ \text { (from Weigelt, 1928) }\end{array} & \text { UPKupf } \\ \begin{array}{l}\text { GFR (Hessen) } \\ \text { (from Zimmermann, 1959) } \\ \text { (rec. from Florin, 1951) }\end{array} & \text { UPKupf } \\ \begin{array}{l}\text { China (Guizhou) } \\ \text { (scheme from Zimmermann, 1959) }\end{array} & \text { UP } \\ \text { China (Guizhou/Yunnan) } & \text { UP } \\ & \\ \text { China (Guangdong) } & \text { UP } \\ \text { Italy (Bolzano) } & \text { UP } \\ \text { GFR (Hessen) } & \text { UPKupf } \\ \text { - } & \text { UPKupf } \\ \text { (from Weigelt, 1928) } & \\ \text { GFR (Hessen) } & \text { UPKupf } \\ \text { GDR (Halle) } & \text { UPKupf } \\ \text { Spain (Gerona) } & \text { LPAutu } \\ \text { Italy (Brescia) } & \text { LPSaxo } \\ \text { China (Guizhou/Yunnan) } & \text { UP } \\ & \\ \text { GDR (Suhl) } & \text { UPZech }\end{array}$

Spain (Soria)

LPAutu

USSR (Krasnoyarskiy Kray Tungusk Basin -)

UP

(scheme)

(C-P)

(from Nejburg, 1960)

USSR (Komi ASSR)

USSR (Komi ASSR)

LPUfim

UPKaza

USSR (Komi ASSR)

LPUfim

China (Chinghai)

UC-LP

China (Kansu)

UC-LP

China Chinghai)

UC-LP 
Venustostrobus Chandra et Surange 1977

$V$. sp.

1977 Chandra et Surange (c), fig.2G,H; pl.1, 7, 8

India $(-)$

$\mathrm{P}$

$V$. diademus Chandra et Surange 1977

1977 Chandra et Surange (e), figs.9-13; pl.4, 19-23; pl.5, 26

1977 Chandra et Surange (e), fig.15A

Remark: descr./ills. include cuticles and seeds. Organic connection with Glossopteris contracta

1978 Surange et Chandra, pl.4, 3

1979 Chandra et Surange, pl.13, $4 ; \mathrm{pl} .22,10$

Remark: organic connection with Glossopteris pantii

$V$. indicus Chandra et Surange 1977

1977 Chandra et Surange (b), figs.1, 2, 5-7; pl.1, 6; pl.2, 714

1977 Chandra et Surange (b), fig.8

Remark: descr./ills. include cuticles.

Organic connection with Glossopteris sp.

1979 Chandra et Surange, pl.14, I

Remark: organic connection with Glossopteris pantii

India (West Bengal) UPRani

(rec.)

(rec.)

India (West Bengal) LGRani

India (West Bengal) LGRani

(rec.)

India (West Bengal) LGRani

\section{Vertebraria Royle 1839 ex McCoy 1847}

$V . \mathrm{sp}$.

1976 Balasundaram et al., pl.22, 9

1976 Banerji et al., pl.2, 24, 25

1976 Teixeira et Pais, fig.35A, D

1977 Gould et Delevoryas, fig.1F, G

1977 Pant, fig. 4

1978 Mussa (c), fig. 1C

1980 Gould et Shibaoka, fig.6C-F

$V$. indica Royle 1839

1976 Gould, fig. $4 \mathrm{D}, \mathrm{E}$

1976 Guerra, figs.1, 2; pls.1-5

1977 Guerra, pl.1, 1-14

1977 Pant, fig.2C

1977 Pant, fig.2D

1977 Pant, fig.2E

1977 Pant, fig. 3A-G

1977 Srivastava (a), fig.5; pl.1, 6

1977 Srivastava (b), pl.2, 13

1980 Retallack, fig.21.5L, M

(scheme)

India (Madhya Pradesh)

UP-LT?

(scheme from Pant, 1962)

(scheme)

India

LG

(rec.)

(scheme)

(scheme)

Brazil (Rio Grande do Sul)

Brazil (Rio Grande do Sul)

LP

(from Pant, 1962)

(from Pant, 1956)

(from Walton et Wilson, 1932)

(from Pant et Singh, 1968)

India (Bihar)

India (Bihar)

LGKarh

Australia (New South Wales)

L-UP

\section{Viatcheslavia Zalessky 1936}

Remark: in text Viatscheslavia. See Farr et al, (1979, p.1847)

$V . \mathrm{sp}$.

1977 Huang, fig. $3 ;$ pl.24, 4

China (Heilongjiang)

$V$. vorcutensis Zalessky 1936

1977 Huang, pl.6, 4-5; pl.39, 1-4

China (Heilongjiang)

UP

\section{Vojnovskya Nejburg 1955}

V. sp.

1976 Durante, pl.56, 3, 4

V. elegans Zimina 1977

1977 Zimina, pl.20, 1--3

1978 Zimina, pl.2, 8

$V$. minima (Khakhlov et Pollak 1935) Nejburg 1961

1976 Durante, pl.56, 2

$V$. pacifica Zimina 1967

1977 Zimina, pl.18, 13, 14; pl.19, 1-9

Mongolia

USSR (Primorskiy Kray)

USSR (Primorskiy Kray)

Mongolia

USSR (Primorskiy Kray)
LP

LP

LP

LP

LP 
V.paradoxa Nejburg 1955

1978 Mamay, fig.1

USSR (Komi ASSR - Pechora

Basin -)

LP

1979 Delevoryas, fig.13

(from Andrews, 1961)

\section{Voltzia Brongniart 1828}

$V . \mathrm{sp}$

1977 Jung, pl.17, 11

Italy (Bolzano)

UP

$V$. hungarica Heer 1876

1977 Jung, pl.17, 2; pl.18, 1, 3

Italy (Bolzano)

UP

$V$. liebeana Geinitz 1862

1978 Pawlowska, fig.3

Poland (Promnik Basin)

UPZech

\section{Voltziopsis Potonié 1899}

V. africana Seward 1934

1976 Gould, fig.4I

1978 Miller, fig. $2 \mathrm{H}$

1980 Retallack, fig. $21.8 \mathrm{H}$

V. angusta (Walkom 1925) Townrow 1967

1980 Retallack, fig.21.8G

$V$. wolganensis Townrow 1967

1980 Retallack, fig.21.8E-F

$\begin{array}{ll}\begin{array}{ll}\text { (scheme from Townrow, 1967) } \\ \text { (rec. from Florin, 1951) }\end{array} & \begin{array}{l}\text { (P-T) } \\ \text { Australia (New South Wales) }\end{array} \\ \begin{array}{ll}\text { UP-LT } \\ \text { Australia (New South Wales) }\end{array} & \text { UP-LT } \\ \text { Australia (New South Wales) } & \text { UP-LT }\end{array}$

\section{Vorcutannularia Pogorevich ex Nejburg 1960}

V. laevis Fefilova 1978

1978 Fefilova (b), fig.20B, G; pl.20, 1; pl.22, 3

1978 Fefilova (b), fig.20A, V, D; pl.20, 2; pl.22, f.4

USSR (Komi ASSR)

UPTata

USSR (Komi ASSR)

UPKaza

$V$ plicata Pogorevich ex Nejburg 1960

1978 Fefilova (b), fig.19; pl.19

1979 Lacey, fig.1(2)

1980 Bold et al., fig.10-47A, B

USSR (Komi ASSR)

LPUfim

(from Nejburg, 1960)

(from Nejburg)

\section{Walchia Sternberg 1825}

W. sp.

1976 Bohlin, fig.614; pl.16, 14 (all cf.)

1978 Gillespie et al., fig. 16

1978 Gillespie et al., pl.54, 1

1978 Gillespie et al., pl.54, 3

W. filiciformia Sternberg 1825

Remark: commonly spelled $W$. filiciformis

1977 Remy et Remy, fig.41A, B

W. geinitzii Florin 1939

1977 Remy et Remy, fig.44A, B

1978 Remy et Remy, (c), pl.7, 10-12; pl.8, 1

W. germanica Florin 1939

1976 Harris, fig. $1 \mathrm{C}$

1977 Remy et Remy, fig.42

1978 Doubinger et al., pl.1, 1; pl.2, 4, 5

1978 Remy et Remy (c), pl.8, 3 (cf.)

1980 Kämpfe, fig.7.13C

$W$. piniformis Sternberg 1825

1976 Daber, fig.475(1)

1976 Denizot, pl.6, 24A-C

1977 Fischer et Gayrard-Valy, fig.61

1979 Schniepp, fig.13

W. stricta Florin 1939

1978 Remy et Remy (c), pl.8, 16 (cf.)

\begin{tabular}{|c|c|}
\hline $\begin{array}{l}\text { China (Kansu) } \\
\text { (rec) }\end{array}$ & UC-LP \\
\hline $\begin{array}{l}\text { USA (West Virginia) } \\
\text { (scheme) }\end{array}$ & $\begin{array}{l}\mathrm{LP} ? \\
(\mathrm{UC}-\mathrm{P})\end{array}$ \\
\hline . & \\
\hline GDR (Gera) & LPAutu \\
\hline Italy (Brescia) & LPSaxo \\
\hline Italy (Brescia) & LPSaxo \\
\hline (scheme) & $(\mathrm{C}-\mathrm{P})$ \\
\hline GDR (Erfurt) & LPAutu \\
\hline Spain (Gerona) & LPAutu \\
\hline $\begin{array}{l}\text { Italy (Brescia) } \\
\text { (scheme from Zimmermann, 1959) }\end{array}$ & LPSaxo \\
\hline (scheme) & \\
\hline $\begin{array}{l}\text { France }(-) \\
\text { (scheme) }\end{array}$ & $\begin{array}{l}\text { LPAutu } \\
\text { (C-P) }\end{array}$ \\
\hline GFR (Saarland) & LPRotl \\
\hline taly (Brescia) & LPSaxo \\
\hline
\end{tabular}


Walchiopremnon Florin 1940

W. sp.

1976 Barthel et al., pl.42, 9

\section{Walchiostrobus Florin 1940}

W. sp.

1976 Harris, fig.1B

1978 Grauvogel-Stamm, fig.44E

1978 Remy et Remy (c), pl.8, 2

1980 Keng, fig. 6

(scheme)

(scheme from Schweitzer. 1963)

Italy (Brescia)

(scheme)

Australia (New South Wales)

UP

1980 Retallack, fig.21.8B, C

\section{Weissites Göppert 1836}

W. pinnatifidus (Gutbier 1835) Remy 1954

1976 Barthel et al., pl.18, 4

1976 Barthel et al., pl.18, 5

\section{Wumengopteris Tian 1980}

W. crassirachis Tian 1980

1980 Tian et Zhang, pl.12, f.1, 5; pl.13, 4, 4A

China (Guizhou)

UP

\section{Xinganphyllum Huang 1977}

$X . \mathrm{sp}$.

1977 Huang, fig.22; pl.6, 3

$X$ aequale Huang 1977

1977 Huang, fig. $20 ;$ pl.6, $1-2 ;$ pl.7, 1-3

$X$. inaequale Huang 1977

1977 Huang, fig.21; pl.27, 2

\section{Yavorskyia Radchenko 1936}

Y. hebetata (Radchenko 1936) Gorelova et Radchenko 1962 1976 Durante, pl.57.2

\section{Zalesskyoxylon Lepekhina et Yatsenko-Khmelevsky 1966}

Z. zambesiensis Maithy 1977

1977 Maithy (a), fig.1; pl.1, 1- 7

Remark: in text Zalesskioxylon

\section{Zamiopteris Schmalhausen 1879}

$Z$. sp.

1976 Bohlin, fig.673(?)

1976 Durante, pl.52, 1

1977 Zimina, pl.7, 1, 2

Z. daubichites Burago 1979

1979 Burago, pl.9, 3; pl.10, 3

Z. dubia Zimina 1974

1976 Durante, pl.53, 24 (ex gr.)

1977 Zimina, fig.15; pl.6, 1-5

Z. glossopteroides Schmalhausen 1879

1977 Huang, pl.41, 2 (cf.)

1977 Zimina, pl.5, 1(?), 3.6

1980 Huang, pl.243, 5 - 6 (cf.)
GDR (Karl-Marx-Stadt)

GDR (Dresden)

LPRotl

LPRotl
Mongolia

UP

Mozambique (Zambesi Basin)

$\mathrm{P}$

$\begin{array}{ll}\text { China (Chinghai) } & \text { UC . LP } \\ \text { Mongolia } & \text { UP } \\ \text { USSR (Primorskiy Kray) } & \text { LP } \\ \text { USSR (Primorskiy Kray) } & \text { LP } \\ \text { Mongolia } & \text { UP } \\ \text { USSR (Primorskiy Kray) } & \text { UP } \\ & \\ \text { China (Heilongjiang) } & \text { LP } \\ \text { USSR (Primorskiy Kray) } & \text { LP } \\ \text { China (Heilongjiang) } & \text { UC-LP }\end{array}$

(C - P)

(UC $\cdots$ P)

LPSaxo 
Z. gorelovae Zimina 1978

1978 Zimina, pl.3, 1-7

1978 Zimina, pl.3, 8-9

Z. lanceolata (Khakhlov et Pollak 1935) Nejburg 1948

1977 Huang, pl.41, 1

1977 Zimina, pl.6, 9 (cf.)

$Z$. rotunda Zimina 1977

1977 Zimina, pl.6, 8(?)

Z. schmalhausenii Shvedov 1940

1979 Burago, pl.10, 4, 5

Z. subglossopteroides Zimina 1974

1977 Zimina, fig.14; pl.5, 2

Z. tailuganensis Gorelova 1962

1977 Huang, pl.24, 3; pl.42, 4-5

\section{Zamites Brongniart 1828}

+Z. kryshtofovichii (Prinada et Radchenko)

1980 Huang, pl.259, 1-12A

\section{Zygopteris Corda 1845}

Z. lacattii Renault 1869

Remark: in text $Z$. lacattei

1980 Bold et al., fig.16-64B

Z. primaria (Cotta 1831) Stenzel 1889

Remark: in text $Z$. primaria (Cotta) Corda

1976 Barthel et al., pl.1, 1-2

\section{Systematic arrangement of the genera}

\section{BRYOPHYTA}

\section{Bachtia (-)}

Bajdaievia (2)

Intia (4)

Jaganovia (1)

Junjagia (1)

Kosjunia (2)

Polyssaievia (1)

Protosphagnum (1)

Ricciopsis (2)

Salairia (2)

Syrjagia (2)

Thallites (-)

Uskatia (3)

Vorcutannularia (2)

\section{EQUISETOPHYTA}

\author{
Annularia (13) \\ Annulina (1) \\ Arthropitys (2) \\ Asterophyllites (3) \\ Bowmanites (-) \\ Calamariophyllum (1) \\ Calamitea (1) \\ Calamites (7)
}

$\begin{array}{ll}\text { USSR (Primorskiy Kray) } & \text { L-UP } \\ \text { USSR (Primorskiy Kray) } & \text { UP } \\ \text { China (Heilongjiang) } & \text { LP } \\ \text { USSR (Primorskiy Kray) } & \text { LP } \\ \text { USSR (Primorskiy Kray) } & \text { LP } \\ \text { USSR (Primorskiy Kray) } & \text { LP } \\ \text { USSR (Primorskiy Kray) } & \text { LP } \\ \text { China (Heilongjiang) } & \text { LP }\end{array}$

China (Heilongjiang)

UP

(rec. from Wettstein)

(C-P)

GDR (Karl-Marx-Stadt)

LPRotl
Calamitina (-)

Calamitopsis (1)

Calamodendron (1)

Calamostachys (3)

Crucicalamites (-)

Dicalamophyllum (2)

Diplocalamites (-)

Equisetites (-)

Equisetum (-)

Gamophyllites (-)

Koretrophyllites (3)

Lelstotheca (1)

Lilpopia (1)

Lobatannularia (9)

Macrostachya (1)

Mesocalamites (1)

Neocalamites (1)

Palaeostachya (2)

Paracalamites (4)

Paracalamostachys (1)

Paraschizoneura (1)

Parasphenophyllum (1)

Paratrizygia (1)

Peltostachys (1)

Phyllotheca (4)

Raniganjia (1)

Sakoarota (1)

Schimperia (-)

Schizoneura (4)

Sphenophyllostachys (-) 
Sphenophyllum (20)

Stellotheca (1)

Stephanophyllites (1)

Stylocalamites (-)

Tchernovia ( )

Trizygia (4)

Umbellaphyllites (2)

\section{LYCOPODIOPHYTA}

Asolanus (2)

Aspidiaria (...)

Bergeria (-)

Bothrodendron (1)

Brasilodendron (2)

Cyclodendron (2)

Knorria (.)

Lepidodendron (17)

Lepidodendropsis ( $\cdots$ )

Lepidophyllum (-)

Lepidostrobus (1)

Lycopodiopsis (4)

Selaginella (1)

Sigillaria (3)

Stigmaria (2)

Subsigillaria (-)

Viatcheslavia (1)

PTEROPHYTA + "PTERIDOPHYLLA"

Abrotopteris (1)

Acitheca (3)

Alethopteris (11)

Angaridium (-)

Angaropteridium (1)

Ankyropteris (1)

Aphlebia (1)

Asansolia (1)

Asterochlaena (1)

Asterotheca (7)

Belemnopteris (3)

Bicoemplectopteridium (1)

Bicoemplectopteris (1)

Bifariusotheca (1)

Biscalitheca (1)

Botrychiopsis (3)

Botryopteris (1)

Brongniartites (-)

Callipteridium (3)

Callipteris (98)

Cardioneura (2)

Cardiopteris (-)

Cathaysiopteris (2)

Caulopteris (1)

Chansitheca (1)

Chiropteris (1)

Cladophlebis (17)

Comia (10)

Compsopteris (7)

Corynepteris (5)
Cyclopteris (1)

Dactylotheca (1)

Damudosorus (1)

Danaeites (7)

Dichotomopteris (2)

Dicksoniites (1)

Dictyopteridium (3)

Diphyllopteris (1)

Discinites (1)

Dizeugotheca (2)

Dunedoonia (1)

Emplectopteridium (1)

Emplectopteris (3)

Eremopteris (2)

Etapteris (1)

Eusphenopteris (2)

Fascipteridium (1)

Fascipteris (6)

Filicites (1)

Gigantonoclea (14)

Gigantopteridium (1)

Gigantopteris (5)

Gondwanidium ( )

Gothanopteris (1)

Grammatopteris (1)

Hagiophyton (-)

Heterangium (-)

Huihuipuia (1)

Iniopteris (1)

Isodictyopteridium (2)

Kashmiropteris (1)

Konnoa (1)

Lepidopteris (1)

Lescuropteris (1)

Lesleya (2)

Linophyllum (1)

Linopteris (1)

Lophotheca (1)

Marattiopsis (1)

Marginopteris (1)

Mariopteris (2)

Millaya (1)

Mixoneura ( $\ldots)$

Nemejcopteris (1)

Neomariopteris (5)

Neurodontopteris ( )

Neurophyllites (1)

Neuropteridium (6)

Neuropteris (10)

Odontopteris (12)

Oligocarpia (-)

Osmundacaulis (1)

Otofolium (2)

Palaeogoniopteris (1)

Palaeosmunda (1)

Palaeovittaria (..)

Pecopteris (60)

Ponsotheca (1)

Prionophyllopteris (1)

Protoblechnum (1) 
Prynadaeopteris (4)

Psaronius (9)

Pseudomariopteris (2)

Psygmophyllum (3)

Ptychocarpus (2)

Pursongia (-)

Rajahia (6)

Renaultia (-)

Reticulopteris (1)

Rhacopteris (1)

Rhaphidopteris (1)

Rhodea (-)

Sagittapteris (1)

Santhalea (1)

Schizostachys (1)

Scolecopteris (11)

Senftenbergia (1)

Sphenopecopteris (1)

Sphenopteridium (1)

Sphenopteris (26)

Sphyropteris (-)

Sporophyllites (1)

Stockmansia (1)

Supaia (5)

Syniopteris (-)

Taeniopteris (19)

Tatarina (6)

Thamnopteris (1)

Thinnfeldia (1)

Tingia (5)

Tricoemplectopteris (1)

Trithecopteris (1)

Tychtopteris (4)

Urnatopteris (-)

Validopteris (1)

Weissites (1)

Wumengopteris (1)

Zamiopteris (9)

Zygopteris (2)

\section{"PTERIDOSPERMAE"}

(incl. Caytoniales, Glossopterales, Pentoxylales)

Antevsia (1)

Arberia (2)

Austroglossa (1)

Callipterianthus (1)

Cistella (2)

Cornuspermum (1)

Crossotheca (2)

Denkania (1)

Dolerotheca (1)

Elatra (1)

Eretmonia (7)

Fertiliger (-)

Fetura (1)

Gangamopteris (10)

Gansucladus (1)

Glossopteris (95)

Glossotheca (3)

\author{
Gnetopsis (-) \\ Hirsutum (-) \\ Indocarpus (-) \\ Jambadostrobus (1) \\ Kendostrobus (1) \\ Lanceolatus (1) \\ Lidgettonia (2) \\ Medullosa (3) \\ Nesowalesia (1) \\ Ottokaria (3) \\ Pachytesta (-) \\ Partha (4) \\ Peltaspermum (2) \\ Pluma (-) \\ Plumsteadia (6) \\ Plumsteadiostrobus (2) \\ Psaliangium (-) \\ Pterispermostrobus (1) \\ Rhabdocarpos (1) \\ Rhabdotaenia (-) \\ Rubidgea (1) \\ Rusangea (1) \\ Scirroma (2) \\ Scopus (4) \\ Scutum (4) \\ Senotheca (1) \\ Squamella (3) \\ Telangium (-) \\ Vannus (-) \\ Venustostrobus (2) \\ Vertebraria (1) \\ CYCADOPHYTA \\ Archaeocycas (1) \\ Cheirophyllum (1) \\ Cycadospadix (1) \\ Nilsonia (2) \\ Noeggerathiopsis (19) \\ Phasmatocycas (1) \\ Plagiozamites (3) \\ Pterophyllum (6) \\ Sphenozamites (1) \\ Yavorsyia (1) \\ Zamites (1)

\section{GINKGOPHYTA} \\ Chapchalia ((1) \\ Ginkgo (-) \\ Ginkgoites (1) \\ Sphenobaiera (5) \\ Trichopitys (2) \\ CONIFEROPHYTA \\ (incl. Cordaitales) \\ Abietopitys (1) \\ Amyelon (-) \\ Araucaria (-)
}


Araucarites (2)

Artisia (1)

Brachyphyllum ()

Cardiocarpus (4)

Carpentiera (1)

Conchophyllum (2)

Cordaianthus (5)

Cordaicladus ( )

Cordaites (17)

Crassinervia (8)

Culmitzschia (1)

Cupressites (1)

Dadoxylon (3)

Distichotheca (1)

Dolianitia (2)

Dorycordaites (..)

Ernestiodendron (1)

Eucordaites ( )

Euryphyllum (1)

Glyptolepis (2)

Gomphostrobus (1)

Henanophyllum (1)

Kamaraspermum ( )

Lebachia (6)

Lepeophyllum (6)

Masculostrobus ( )

Moyliostrobus (1)

Paracordaites ( )

Paranocladus (1)

Phyllocladopitys (1)

Poa-Cordaites ( )

Protopodocarpitys (1)

Pseudovoltzia (1)

Quadrocladus (1)

Rhipidopsis (14)

Rufloria (21)

Sashinia (1)

Schuetzia (1)

Scleromedulloxylon (2)

Sparsistomites (1)

Strigillotheca (1)

Taxopitys (3)

Tylodendron (1)

Ullmannia (3)

Voltzia (2)

Voltziopsis (3)

Walchia (5)

Walchiopremnon (")

Walchiostrobus ( )

Walkomiella (1)

\section{SPERMATOPHYTA INCERTAE SEDIS AND PROBLE- MATICA}

Ammatophyllum( )

Antarticoxylon (1)

Araucarioxylon (1)

Arberiella (2)

Arberiopsis (1)

Australoxylon (1)
Austroscleromedulloxylon (1)

Bageopitys (1)

Barakaroxylon (5)

Bardocarpus (1)

Brasilestiloxylon (1)

Buriadia (1)

Cardiolepis (1)

Carpolithes (5)

Cathaysioxylon (1)

Caulophyllites (1)

Chulakia (2)

Cladostrobus (1)

Cordaicarpus (5)

Cornucarpus (2)

Damudoxylon (1)

Dicranophyllum (3)

Dzungariella (2)

Eichwaldia (.)

Gaussia (1)

Gigantospermum (.)

Ginkgophyllum (3)

Ginkgophyton (3)

Ginkgophytopsis (2)

Glottophyllum (2)

Guizhoua (1)

Holcospermum (1)

Kawizophyllum (1)

Laxostrobus (-)

Lopadiangium (1)

Mooia (1)

Myelontordoxylon (2)

Myeloxylon (1)

Nephropsis (9)

Niazonaria (-)

Noeggerathia (1)

Nucicarpus (1)

Nummulospermum (-)

Palaeospiroxylon (1)

Palaeoxylon (.)

Palmatophyllites (1)

Parataxopitys (1)

Paratordoxylon (1)

Pelourdea (1)

Phylladoderma (3)

Platycardia (1)

Polysolenoxylon (3)

Poroxylon (1)

Protophyllocladoxylon ()

Pseudorhipidopsis (1)

Pterygospermum (1)

Radicites (.)

Rhizomopsis (1)

Rigbya (1)

Samaropsis (9)

Sandrewia (1)

Skokia (1)

Slivkovia (1)

Solenobrasilioxylon (1)

Squamocarpus (1)

Stephanostoma (1) 
Sylvella $(\cdots)$

Synia (1)

Tingiostachya (-)

Tordoxylon (2)

Trigonocarpum (2)

\section{Systematic arrangement of new taxa}

\section{BRYOPHYTA}

Bajdaievia longiuscula Fefilova 1978 speciosa Fefilova 1978

Intia vicaria Fefilova 1978

\section{Jaganovia Radchenko et Verbitskaya 1980}

Jaganovia ziminkensis Verbitskaya 1980

Ricciopsis anakitensis Verbitskaya 1980 parua Verbitskaya 1980

Salairia confinis Fefilova 1976 singularis 1976

Uskatia dentata Fefilova 1978 vicina Fefilova 1978

Vorcutannularia laevis Fefilova 1978

\section{EQUISETOPHYTA}

\section{Calamitopsis Remy et Remy ex Remy et Remy 1978}

Koretrophyllites gracilis Verbitskaya 1980 latifolius Verbitskaya et Radchenko 1980

Lobatannularia cathaysiana Yao 1980 fusiformis Zhang 1978 huapingensis Feng 1977 longifolia Feng 1977

Parasphenophyllum crenulatum Maithy 1978

\section{Peltostachys Barthel, Götzelt et Urban 1976}

Peltostachys altendorfense Barthel, Götzelt et Urban 1976 Raniganjia qubuensis Hsü 1976

\section{Sakoarota Appert 1977}

Sakoarota polyangiata Appert 1977

Schizoneura brevifolia Yao 1980 lingshanensis Feng et Meng 1977

Sphenophyllum elagatiefolium Xi 1977 gongxianense Xi 1977 meyenii Zimina 1977 osipoviense Zimina 1977 sakoense Appert 1977 sino-coreanum Yabe 1920 ssp. dentatum Zhang 1978 yiyangense Xi 1977 yujiaense Huang 1980
Tubicaulis (1)

Tungussocarpus (1)

Vojnouskya (4)

Xinganphyllum (2)

Zalesskyoxylon (1)

\section{Stephanophyllites Millan et Dolianiti 1977}

Stephanophyllites sanpaulensis Millan et Dolianiti 1977 Umbellaphyllites nacumilensis Verbitskaya 1980

\section{LYCOPODIOPHYTA}

Asolanus chapchaliensis Bohlin 1976 Bothrodendron henanense Xi 1977

Brasilodendron africanum Lemoigne et Brown 1980

Cyclodendron brasiliensis Lejal-Nicol et Bernardes de Oliveira 1979

Lepidodendron henanense Feng 1977 latiligulatum Bohlin 1976 lepidophloides Yao 1980 pingdingshanense Xi 1977 xingningense Feng 1977 xuanweiense Yao 1980

Lepidostrobus acutisquamus Yao 1980

Lycopodiopsis costilatus Lemoigne et Brown 1980

PTEROPHYTA + "PTERIDOPHYLLA"

\section{Abrotopteris Mo 1980}

Acitheca unifurcata Yang et Chen ex Zhang 1978 Alethopteris firmata Zhang 1978 yiyangensis Feng 1977

\section{Asansolia Pant et Misra 1976}

Asterotheca golondrinensis Herbst 1978 primorskiensis Burago 1980

Belemnopteris pellucida Pant et Choudhury 1977 sagittifolia Pant et Choudhury 1977

\section{Bifariusotheca Zhao 1980}

Bifariusotheca qinglongensis Zhao 1980 Callipteris biforma Huang 1977 conferta (Sternberg 1833) Brongniart 1849 var. supaioides Haubold 1980 heilongjiangensis Huang 1977 hongshanensis Huang 1977 obese Huang 1977 pseudoshenshuensis Huang 1977 shenshuensis Huang ex Huang 1976 tangwangheensis Huang 1977 tongxingensis Huang 1980 Cathaysiopteris huapingensis Feng 1977 Cladophlebis connexa Zhang 1978 fuyuanensis Zhang 1980 
gorskii Vladimirovich 1980

heishanensis Bohlin 1976

henanensis Zhang et Mo 1979

licharevii Vladimirovich 1980

melnikovii Burago 1980

mongolica Durante 1976

parapermica Chang MS ex Zhang 1978

xiaohinganensis Huang 1980

yunnanica Zhang 1980

Comia cangfanggouensis $\mathrm{Hu} 1980$

microphylla Huang 1977

multinervis Huang 1977

obese Huang 1977

shenshuensis Huang 1977

tenueaxis Huang 1977

Compsopteris guangdongensis Feng 1977 punctinervis Mo 1980

Corynepteris kansuensis Bohlin 1976 parilobata Bohlin 1976 plicata Bohlin 1976

\section{Damudosorus Pant et Misra 1977}

Damudosorus searsolensis Pant et Misra 1977

Danaeites guangdongensis Feng 1977 guangxingense Huang 1980 qujiangensis Feng 1977

Dichotomopteris ovata Maithy 1977 qubuensis Hsü 1976

Dictyopteridium flabellatum Benecke 1976

\section{Diphyllopteris Srivastava 1978}

Diphyllopteris verticillata Srivastava 1978

Dizeugotheca bortoluzzii Cazzulo-Klepzig 1978 qubuensis Hsü 1976

\section{Dunedoonia Holmes 1977}

Dunedoonia reticulata Holmes 1977

Emplectopteris neimongolensis Huang 1976 sinensis Huang 1976

\section{Fascipteridium Zhang et Mo 1980}

Fascipteridium ellipticum Zhang et Mo 1979

Fascipteris kaishantunensis Huang 1980

Gigantonoclea microphylla Huang 1980 minor Feng 1977 plumosa Mo 1980 tieyingensis Huang 1980 unita Huang 1980 yujiaensis Huang 1980

Gigantopteris meganetes Tian 1980 paradoxa Mo 1980

\section{Huihuipuia Bohlin 1976}

Huihuipuia reticulata Bohlin 1976

\section{Kashmiropteris Kapoor 1979}

Kashmiropteris meyenii Kapoor 1979

Lesleya gimmii Remy et Remy 1978

\section{Linophyllum Zhao 1980}

Linophyllum xuanweiense Zhao 1980

Linopteris semianastomosa Tenchov 1977

\section{Lophotheca Zhao 1980}

Lophotheca panxianensis Zhao 1980 Marattiopsis orientalis Burago 1977

\section{Marginopteris Sal'menova 1978}

Marginopteris kasachstanica Sal'menova 1978 Mariopteris crassipinnulata Bohlin 1976 yuemenensis Bohlin 1976

\section{Millaya Mapes et Schabilion 1979}

Millaya tularosana Mapes et Schabilion 1979 Neomariopteris barakarensis Srivastava 1978 khanii Maithy 1977

\section{Neurophyllites Zhang 1980}

Neurophylites pecopteroides Zhang 1980

Neuropteridium guizhouense Zhang 1980 guizhouensis Zhang 1980 tieyingensis Huang 1980

Neuropteris dagangensis Huang 1980 daheshenensis Huang 1980

Odontopteris henanensis Xi 1977 xinganensis Huang 1977 yiyangensis Feng 1977

Osmundacaulis stumkei Erasmus 1977 (?)

Pecopteris anbangensis Huang 1977 deducans Huang 1977 dissimilaris Burago 1980 elegantula Zhang 1980 fuyuanensis Zhang 1980 guizhouensis Chang MS ex Zhang 1978 hongshanensis Huang 1977 lingulata Zhang 1980 longifolioides Zhang 1980 mucronata Durante 1976 niamdensis Zalessky et Chirkova 1937 fa. makuchinae Burago 1976 opposita Cazzulo-Klepzig 1978 punicoides Zhang et Mo 1979 qingyunensis Zhang 1980 shuichengensis Zhang 1978 tangwangheensis Huang 1980 tumenjiangensis Huang 1980

\section{Ponsotheca Bernardes de Oliveira 1980}

Ponsotheca roesleri Bernardes de Oliveira 1980 Prynadaeopteris minuta Vladimirovich 1980 rhomboidea Vladimirovich 1980 
Prionophyllopteris Mo 1980

Prionophyllopteris spiniformis Mo 1980

Psygmophyllum ussuriense Burago 1976

Rajahia guizhouensis Zhang 1980

major Zhang 1980

minor Zhang 1980

Rhaphidopteris praecursoria Meien 1979

Santhalea Maithy 1977

Santhalea bansloiensis Maithy 1977

Senftenbergia saxonica Barthel, Götzelt et Urban 1976

Sphenopecopteris Zhang et Mo 1979

Sphenopecopteris beaniata Zhang et Mo 1979

Sphenopteris gongxianensis Xi 1977

heilongjiangensis Huang 1977

huihuipuensis Bohlin 1976

sellardsii Baxter 1978

yichunensis Huang 1977

\section{Stockmansia Bohlin 1976}

Supaia shenshuensis Huang 1977

tieliensis Huang 1977

Taeniopteris crassinervis Mo 1980 guangxingensis Huang 1980 primorjensis Burago 1978 punctifera Appert 1977 rarinervis Zhao 1980 sinegorkiensis Burago 1978

Tatarina conspicua Goman'kov et Meien 1979 lobata Meien 1980

pinnata Goman'kov et Meien 1979 sadounikovii Meien 1980 sinuosa Goman'kov 1980 verrucosa Goman'kov 1980

Tingia yichuanensis Feng 1977

\section{Trithecopteris Pant et Misra 1977}

Trithecopteris gondwanensis Pant et Misra 1977

Tychtopteris arunensis Huang 1980 densinervis $\mathrm{Hu} 1980$ obesa $\mathrm{Hu} 1980$

Validopteris kokonoriensis Bohlin 1976

\section{Wumengopteris Tian 1980}

Wumengopteris crassirachis Tian 1980.

Zamiopteris daubichites Burago 1979 gorelovae Zimina 1978 rotunda Zimina 1977

\section{"PTERIDOSPERMAE"}

(incl. Caytoniales, Glossopterales, Pentoxylales)

\section{Elatra Appert 1977}

E. bella Appert 1977

Fertiliger Schopf 1976

\section{Fetura Benecke 1976}

\section{Gansucladus Bohlin 1976}

Gansucladus parvifolia Bohlin 1976

Glossopteris andreanskyi Kovács-Endrödy 1977 bosei Chandra et Surange 1979 churiensis Srivastava 1977 karharbariensis Chandra et Surange 1979 maccoyi Kovács-Endrödy 1977 mohudaensis Chandra et Surange 1979 nakkarea Chandra et Surange 1979 nimishea Chandra et Surange 1979 pantii Chandra et Surange 1979 pseudocommunis Kovács-Endrödy 1976 raniganjensis Chandra et Surange 1979 rewaensis Chandra et Surange 1979 saksenae Chandra et Surange 1979 syaldiensis Chandra et Surange 1979 taeniensis Chandra et Surange 1979 talchirensis Chandra et Surange 1979

Glossotheca immanis Chandra et Surange 1977

Jambadostrobus Chandra et Surange 1977

Jambadostrobus pretiosus Chandra et Surange 1977

\section{Nesowalesia Pant 1977}

Nesowalesia edwardsii Pant 1977

Ottokaria biharensis Srivastava 1978 raniganjensis Banerjee 1978

Partha belmontensis White 1978 sessilis White 1978

Peltaspermum buevichae Goman'kov et Meien 1979 Plumsteadia lanceolata Srivastava 1978 semnes Rigby 1978

\section{Plumsteadiostrobus Chandra et Surange 1977}

Plumsteadiostrobus ellipticus Chandra et Surange 1977 pretiosus Chandra et Surange 1977

Rhabdocarpos olivaeformis Mo 1980

Rubidgea emarginata Maithy 1977

\section{Scirroma Chandra et Surange 1977}

Scirroma angusta Chandra et Surange 1977 ventilebra Chandra et Surange 1977

\section{Scopus Benecke 1976}

Scopus confertus Benecke 1976 didiscus Benecke 1976 
gibbosus Benecke 1976

obscurus Benecke 1976

\section{Squamella White 1978}

Squamella ampla White 1978 australis White 1978 ovulifera White 1978

\section{Venustostrobus Chandra et Surange 1977}

Venustostrobus diademus Chandra et Surange 1977 indicus Chandra et Surange 1977

\section{CYCADOPHYTA}

\section{Cheirophyllum Pant et Singh 1978}

Cycadospadix yochelsonii Mamay 1976 Nilsonia hongshanensis Huang 1977

Noeggerathiopsis lianzhushanensis Huang 1980 mishanensis Huang 1980 tieliensis Huang 1977 xinganensis Huang 1977

Pterophyllum fromagetii Vozenin-Serra 1979

Sphenozamites permicus Burago 1977

\section{GINKGOPHYTA}

\section{Chapchalia Bohlin 1976}

Chapchalia tsaidamensis Bohlin 1976 Ginkgoites xinhuaensis Feng 1977 Sphenobaiera biloba Feng 1977 macrofolia Burago 1977

\section{CONIFEROPHYTA}

(incl. Cordaitales)

Cardiocarpus cardioformis Verbitskaya 1980 Conchophyllum tsaidamense Bohlin 1976

Cordaites buragoi Zimina 1977 odontophyllus Glukhova 1978 primorskiensis Zimina 1976 tologoiensis Durante 1976 torulosus Glukhova 1980 yujiaensis Huang 1980

Crassinervia grammii Zimina 1977 mishanensis Huang 1980 strelokensis Zimina 1977

Dadoxylon malaimbandense Marguerier 1976 Dolianitia madagascariensis Appert 1977

\section{Henanophyllum Xi et Feng 1977}

Henanophyllum palamifolium Xi et Feng 1977 Lepeophyllum liaoningense Huang 1980 ovatum $\mathrm{Hu} 1980$

\section{Protopodocarpitys Mussa 1976}

Protopodocarpitys roesleri Mussa 1976 Quadrocladus dvinensis Meien 1978 Rhipidopsis guizhouensis Tian 1980 hongshanensis Huang 1977 lobulata Mo 1980 minor Feng 1977 minutus Zhang 1978 multifurcata Tian 1980 shifaensis Huang 1980 schuichengensis Tian 1980 langwangheensis Huang 1980 xinganensis Huang 1977

Rufloria bakanasica Sal'menova 1978 ilimpejica Glukhova 1976 kerulenica Durante 1976 lepeophylloides Durante 1976 magnifolia Durante 1976 marginata Men'shikova 1980 mira Glukhova et Men'shikova 1980 seljatitskii Men'shikova 1980 sublanceolata Zimina 1976 tomentella Glukhova 1976 ulannurica Durante 1976 ussurica Zimina 197 ; vassilyevii Zimina 1977

\section{Sashinia Meien 1978}

Sashinia aristovensis Meien 1978 Scleromedulloxylon batoviense Guerra 1976 Sparsistomites communis Glukhova 1980 Taxopitys indica Prasad et Chandra 1978

SPERMATOPHYTA INCERTAE SEDIS AND PROBLEMATICA

Antarticoxylon sewardii Mussa 1980 Araucarioxylon loharense Agashe et Gowda 1978

\section{Arberiopsis Bernardes de Oliveira 1978}

Arberiopsis boureaui Bernardes de Oliveira 1978 Australoxylon kanhargaoense Prasad et Chandra 1978

\section{Austroscleromedulloxylon Mussa 1980}

Austroscleromedulloxylon geraldinii Mussa 1980

\section{Bageopitys Dohms 1976}

Bageopitys articulata Dohms 1976 Barakaroxylon resiniferum Guerra 1978

\section{Brasilestiloxylon Mussa 1978}

Brasilestiloxylon piracicabense Mussa 1978 Carpolithes glansiformis Mo 1980 minutus Zhao 1980 punctatus Zhao 1980 speculatus Mo 1980 
Cathaysioxylon Vozenin-Serra 1978

Caulophyllites Pant et Singh 1979

Caulophyllites indica Pant et Singh 1979

\section{Chulakia Sal'menova 1979}

Chulakia alveolata Sal'menova 1979 pilifera Sal'menova 1979

Cordaicarpus irapuensis Bernardes de Oliveira 1977 madagascariensis Appert 1977 rocha-camposii Bernardes de Oliveira 1977 trapezoideus Verbitskaya 1980

Cornucarpus cerquilhensis Millan 1977

Damudoxylon iratiensis Guerra-Sommer 1977

Dzungariella fucoides Sal'menova 1979 kizilkainarica Sal'menova 1979

Ginkgophyllum zhongguoense Feng 1977

Ginkgophyton giganteum Burago 1976

Ginkgophytopsis gigantea (Burago 1976) Burago 1977 xinganensis Huang 1977

Guizhoua Zhao 1980

Guizhoua gregalis Zhao 1980

Kawizophyllum Kapoor 1979

Kawizophyllum dunpathriensis Kapoor 1979

Lopadiangium Zhao 1980

Lopadiangium acmodontum Zhao 1980

\section{Geographical distribution and chronostratigraphy of the taxa}

\section{Antarctica (-)}

\section{Permian (unspecified)}

Eretmonia sp.

Glossopteris sp.

Nummulospermum sp.

\section{Lower Gondwana}

Glossopteris indica

\section{Argentina (Buenos Aires)}

Permian (unspecified)

Ottokaria bengalensis

\section{Argentina (Chubut)}

Permian (unspecified)

Lycopodiopsis derbyi
Myelontordoxylon Mussa 1978

Myelontordoxylon brasiliense Mussa 1978 vittii Mussa 1978

Nephropsis elegans Huang 1980 pseudosimilis Burago 1979

Palaeospiroxylon Prasad et Chandra 1980

Palaeospiroxylon heterocellularis Prasad et Chandra 1980

\section{Paratordoxylon Mussa 1978}

Paratordoxylon camposi Mussa 1978

Pelourdea contracta Mo 1980

Phylladoderma aequalis Meien 1977 stenuifolia Sal'menova 1978

Samaropsis dolianitii Millan 1977 millaniana Bernardes de Oliveira 1977 rigbyi Millan 1977 sancti-marci Bernardes de Oliveira 1977

\section{Solenobrasilioxylon Mussa 1978}

Solenobrasilioxylon irinei Mussa 1978

\section{Squamocarpus Mo 1980}

Squamocarpus papilioformis Mo 1980 Tordoxylon sanpaulense Mussa 1978 Trigonocarpum laguncula Bohlin 1976 Vojnouskya elegans Zimina 1977

\section{Xinganphyllum Huang 1977}

Xinganphyllum aequale Huang 1977 inaequale Huang 1977

Zalesskyoxylon zambesiensis Maithy 1977

\section{Lower Permian}

Lycopodiopsis millanii

Lycopodiopsis pedroanus

\section{Argentina (La Rioja)}

Permian (unspecified)

Lycopodiopsis derbyi

\section{Lower Permian}

Lycopodiopsis millanii

Lycopodiopsis pedroanus

\section{Argentina (San Juan)}

\section{Lower Permian}

Lycopodiopsis pedroanus

\section{Argentina (San Luis)}

\section{Lower Permian}

Euryphyllum whittianum 


\section{Argentina (Santa Cruz)}

\section{Lower Permian}

Asterotheca golondrinensis

\section{Australia (-)}

\section{Permian (unspecified)}

Gangamopteris sp.

Glossopteris browniana

Thamnopteris sp.

\section{Australia (New South Wales)}

\section{Permian (unspecified)}

\section{Cistella ampla}

Cordaites spathulata

Glossopteris ampla

Glossopteris angustifolia

Glossopteris browniana

Glossopteris clarkei

Glossopteris communis

Glossopteris conspicua

Glossopteris cordata

Glossopteris elongata

Glossopteris formosa.

Glossopteris indica

Glossopteris intermittens

Glossopteris linearis

Glossopteris retusa

Neomariopteris lobifolia

Phyllotheca australis

Samaropsis sp.

Samaropsis goraiensis

Scutum sp.

Scutum sahnil

\section{Upper Permian}

Austroglossa walkomii

Brachyphyllum sp.

Cyclodendron leslii

Dictyopteridium sporiferum

Dunedoonia reticulata

Eretmonia sp.

Eretmonia cooyalensis

Eretmonia natalensis

Gangamopteris cyclopteroides

Gangamopteris major

Gangamopteris spathulata

Glossopteris acuta

Glossopteris ampla

Glossopteris conspicua

Glossopteris linearis

Glossopteris parallela

Glossopteris rectinervis

Glossopteris spathulato-cordata

Glossopteris taeniopteroides
Glossopteris wilkinsonii

Isodictyopteridium costatum

Lelstotheca robusta

Lidgettonia $\mathrm{sp}$.

Neomariopteris lobifolia

Noeggerathiopsis spathulata

Ottokaria sp.

Partha belmontensis

Partha sessilis

Phyllotheca australis

Rigbya arberioides

Rusangea elegans

Scutum sp.

Squamella ampla

Squamella australis

Squamella ovulifera

Selaginella harrisiana

Senotheca murulidihensis

Thinnfeldia callipteroides

Vannus sp

Vertebraria indica

Voltziopsis africana

Voltziopsis angusta

Voltziopsis wolganensis

Walkomiella australis

\section{Lower Permian}

Austroglossa walkomii

Botrychiopsis ovata

Botrychiopsis plantiana

Botrychiopsis weissiana

Cyclodendron leslit

Eretmonia cooyalensis

Gangamopteris cyclopteroides

Gangamopteris major

Gangamopteris spathulata

Glossopteris clarkei

Glossopteris conspicua

Glossopteris elegans

Glossopteris linearis

Lelstotheca robusta

Neomariopteris lobifolia

Noeggerathiopsis spathulata

Phyllotheca australis

Selaginella harrisiana

Vertebraria indica

\section{Lower Gondwana}

Glossopteris angustifolia

Glossopteris browniana.

Glossopteris indica

Nesowalesia edwardsii

\section{Australia (Queensland)}

\section{Permian (unspecified)}

Cistella ampla 


\section{Upper Permian}

Arberiella sp.

Belemnopteris elongata

Glossopteris sp.

Glossopteris communis

Glossopteris indica

Isodictyopteridium walkomii

Plumsteadia ampla

Plumsteadia microsacca

Plumsteadia ovata

Rigbya arberioides

\section{Lower Permian}

Plumsteadia semnes

\section{Australia (Victoria)}

\section{Permian (unspecified)}

Glossopteris cyclopteroides

Glossopteris maccoyi

Glossopteris obliqua

\section{Austria (Carinthia)}

\section{Lower Permian}

Callipteris conferta

\section{Brazil (Piaui)}

\section{Permian (unspecified)}

Psaronius brasiliensis

\section{Brazil (Paraná)}

\section{Upper Permian}

Dizeugotheca sp.

Sphenophyllum speciosum

\section{Lower Permian}

Asterotheca sp.

Paranocladus fallax

Pecopteris cambuhyensis

Tylodendron speciosum

\section{Brazil (Rio Grande Do Sul)}

Permian (unspecified)

Bageopitys articulata

Glossopteris obovata

Polysolenoxylon bageense

Polysolenoxylon bortoluzzii

Polysolenoxylon whitei

Scleromedulloxylon batoviense

Vertebraria indica

\section{Upper Permian}

Barakaroxylon brasiliense

Barakaroxylon resiniferum

Damudoxylon iratiensis

\section{Middle Permian}

Damudoxylon iratiensis

\section{Lower Permian}

Botrychiopsis sp.

Botrychiopsis plantiana (LPSakm)

Brasilodendron pedroanum

Buriadia sp. (LPSakm)

Buriadia heterophylla (LPSakm, LPSakm-Arti)

Chiropteris sp. (LPSakm)

Chiropteris reniformis (LPSakm)

Cordaicarpus sp. (LPSakm)

Cordaites sp. (LPSakm)

Cordaites hislopii (LPSakm)

Cornucarpus patagonicus (LPSakm, LPSakm-Arti)

Fertiliger sp. (LPSakm)

Gangamopteris sp. (LPSakm)

Gangamopteris angustifolia (LPSakm, LPSakm-Arti)

Gangamopteris buriadica (LPSakm, LPSakm-Arti)

Gangamopteris obovata (LPSakm, LPSakm-Arti)

Ginkgophyllum criciumensis (LPSakm, LPSakm-Arti)

Glossopteris sp. (LPSakm)

Glossopteris indica (LPSakm, LPSakm-Arti)

Koretrophyllites sp. (LPSakm)

Nephropsis sp. (LPSakm-Arti)

Noeggerathiopsis hislopii (LPSakm-Arti)

Ottokaria sp.

Paracalamites australis (LPSakm, LPSakm-Arti)

Rubidgea sp. (LPSakm)

Samaropsis sp. (LPSakm)

Samaropsis seixasi (LPSakm, LPSakm-Arti)

Vertebraria indica

\section{Brazil (Santa Catarina)}

Permian (unspecified)

Chiropteris reniformis

\section{Upper Permian (all UPKaza-Tata)}

Dizeugotheca bortoluzzii

Glossopteris indica

Noeggerathiopsis hislopii

Paracalamites australis

Pecopteris sp.

Pecopteris opposita

Pecopteris pedrasica

Schizoneura gondwanensis

Sphenopteris alata

Sphenopteris lobifolia

\section{Middle Permian}

Arberia sp.

Arberia brasiliensis 
Arberia minasica

Arberiopsis sp.

Arberiopsis boureaui

\section{Lower Permian}

Arberia sp.

Arberia brasiliensis

Arberia minasica

Arberiopsis sp.

Arberiopsis boureaui

Cordaicarpus sp. (LPArti)

Cordaicarpus irapuensis (LPArti)

Cordaicarpus rocha-camposii (LPArti)

Cordaicarpus zeilleri (LPArti)

Cyclodendron brasiliensis

Noeggerathiopsis sp. (LPArti)

Noeggerathiopsis hislopii (LPArti)

Ponsotheca roesleri (LPArti Kung)

Samaropsis sp. (LPArti)

Samaropsis millaniana (LPArti)

Samaropsis sancti-marci (LPArti)

\section{Brazil (São Paulo)}

\section{Permian (unspecified)}

Antarticoxylon sewardil

Artisia sp.

Austroscleromedulloxylon geraldinii

Brasilestiloxylon piracicabense

Polysolenoxylon sp.

Protopodocarpitys roesleri

Solenobrasilioxylon irine

\section{Upper Permian}

Myelontordoxylon brasiliense

Myelontordoxylon vittii

Paratordoxylon camposi

Tordoxylon sanpaulense

\section{Middle Permian}

Myelontordoxylon brasiliense

Myelontordoxylon vittii

Paratordoxylon camposi

Tordoxylon sanpaulense

\section{Lower Permian}

Cornucarpus cerquilhensis

Fertiliger sp.

Phyllotheca australis

Samaropsis dolianitii

Samaropsis rigbyi

Stephanophyllites sanpaulensis

\section{Bulgaria (Northwest)}

\section{Lower Permian}

Callipteris conferta

Callipteris lodevensis
Callipteris naumannii

Callipteris nicklesii

Ernestiodendron filiciformia

Gomphostrobus bifidus

Lebachia frondosa

Lebachia parvifolia

Lebachia piniformis

Linopteris semianastomosa

Neuropteris auriculata

Neuropteris planchardii

Sphenopteris fossorum

\section{Cambodia (-)}

\section{Upper Permian}

Cathaysioxylon khmerinum (UPKaza)

\section{Canada (-)}

\section{Permian}

Lepidodendropsis sp. (?)

\section{Chile (Valdivia)}

Lower Permian

Glossopteris sp. (LP?)

Neuropteris sp. (LP?)

\section{China (-)}

\section{Permian (unspecified)}

Gigantonoclea lagrelii

Konnoa penchihuensis

\section{Upper Permian}

Emplectopteris triangularis

Konnoa penchihuensis

\section{Lower Permian}

Lepidodendron sp.

\section{China (Chinghai)}

\section{Lower Permian}

Ammatophyllum sp. Angaridium sp.

Annularia sp.

Asolanus chapchaliensis

Asterophyllites sp.

Calamites $\mathrm{sp.}$

Cardiopteris sp.

Chapchalia sp.

Chapchalia tsaidamensis 
Conchophyllum sp.

Conchophyllum tsaidamense

Cordaites sp.

Corynepteris sp.

Corynepteris parilobata

Corynepteris plicata

Corynepteris sternbergii

Corynepteris tridentata

Glossopteris sp.

Lepidodendron $\mathrm{sp}$.

Lepidodendron latiligulatum

Neuropteris sp.

Odontopteris laceratifolia

Pecopteris sp.

Plagiozamites sp.

Rhabdocarpus sp.

Rhodea sp.

Schizoneura sp.

Sphenophyllum $\mathrm{sp}$.

Sphenopteris sp.

Taeniopteris sp.

Tingia sp.

Validopteris sp.

Validopteris kokonoriensis

Zamiopteris sp.

\section{China (Fukien)}

\section{Upper Permian}

Bicoemplectopteris hallei

\section{China (Guangdong)}

\section{Upper Permian}

Annularia pingloensis Asterotheca cottoni Cathaysiopteris huapingensis Compsopteris contracta Compsopteris guangdongensis Danaeites guangdongensis Danaeites rigida Danaeites qujiangensis Fascipteris hallei Fascipteris stena Gigantonoclea acuminatiloba Gigantonoclea minor Gigantopteris dictyophylloides Ginkgophyllum zhongguoense Lepidodendron acutangulum Lepidodendron oculus-felis Lepidodendron xingningense Lobatannularia heianensis Lobatannularia huapingensis Lobatannularia longifolia Lobatannularia multifolia Neuropteridium coreanicum
Neuropteridium polymorphum

Otofolium ovatum

Otofolium polymorphum

Paracalamites stenocostatus

Pecopteris anderssonii

Pecopteris arborescens

Pecopteris hemitelioides

Pecopteris hirta

Pecopteris orientalis

Pecopteris wongii

Rhipidopsis panii

Sphenopteridium pseudogermanicum

Stigmaria rugulosa

Tingia carbonica

Ullmannia bronnii

Ullmannia frumentaria

\section{China (Guangxi)}

\section{Upper Permian}

Gigantonoclea sp.

Neuropteridium nervosum

Schizoneura lingshanensis

Sigillaria ichthyolepis

Ullmannia bronnii

\section{Ching (Guizhou)}

\section{Upper Permian}

Abrotopteris guizhouensis Acitheca cupressoides Acitheca unifurcata Alethopteris firmata Amyelon sp. Annularia pingloensis Annularia shirakii

Bifariusotheca qinglongensis Calamites sp.

Cardiocarpus sp.

Carpolithes sp.

Carpolithes glansiformis

Carpolithes minutus

Carpolithes punctatus

Carpolithes speculatus

Chansitheca sp.

Chansitheca kidstonii

Cladophlebis connexa

Cladophlebis fuyuanensis

Cladophlebis ozakii

Cladophlebis parapermica

Cladophlebis permica

Compsopteris sp.

Compsopteris contracta

Compsopteris imparis

Compsopteris wongii

Compsopteris punctinervis 
Cornucarpus sp.

Cladophlebis ozakii

Cladophlebis parapermica

Cladophlebis permica

Cladophlebis yunnanica

Danaeites sp.

Danaeites mirabilis

Danaeites rigida

Danaeites saraepontanus

Discinites orientalis

Distichotheca crossothecoides

Fascipteris densata

Fascipteris hallei

Fascipteris stena

Gigantonoclea dictyophylloides

Gigantonoclea guizhouensis

Gigantonoclea hallei

Gigantonoclea lagrelii

Gigantonoclea longifolia

Gigantonoclea plumosa

Gigantonoclea rosulata

Gigantopteris cordata

Gigantopteris dictyophylloides

Gigantopteris meganetes

Gigantopteris nicotianaefolia

Gigantopteris paradoxa

Gigantospermum sp.

Glossopteris guizhouensis

Guizhoua gregalis

Heterangium sp.

Knorria sp.

Lepidodendron sp.

Lepidodendron acutangulum

Lepidodendron lepidophloides

Lepidodendron oculus-felis

Lepidodendron xuanweiense

Lepidostrobus acutisquamus

Linophyllum xuanweiense

Lobatannularia cathaysiana

Lobatannularia fusiformis

Lobatannularia heianensis

Lobatannularia lingulata

Lobatannularia multifolia

Lopadiangium acmodontum

Lophotheca panxianensis

Neurophyllites pecopteroides

Neuropteridium coreanicum

Neuropteridium guizhouense

Neuropteridium guizhouensis

Nilsonia sp.

Otofolium ovatum

Paracalamites sp.

Paracalamites stenocostatus

Pecopteris arcuata

Pecopteris cyathea

Pecopteris echinata

Pecopteris elegantula

Pecopteris fuyuanensis

Pecopteris guizhouensis

Pecopteris hemitelioides
Pecopteris heteropinna

Pecopteris lativenosa

Pecopteris lingulata

Pecopteris longifolioides

Pecopteris marginata

Pecopteris norinii

Pecopteris polymorpha

Pecopteris qingyunensis

Pecopteris sahnii

Pecopteris shuichengensis

Pecopteris taiyuanensis

Pelourdea contracta

Phyllotheca etheridgei

Plagiozamites sp.

Plagiozamites linearis

Plagiozamites oblongifolius

Prionophyllopteris spiniformis

Psaronius sp.

Psaronius sinensis

Pterophyllum eratum

Rajahia guizhouensis

Rajahia major

Rajahia minor

Rajahia mirabilis

Rajahia rigida

Rhabdocarpos olivaeformis

Rhipidopsis sp.

Rhipidopsis ginkgoides

Rhipidopsis gondwanensis

Rhipidopsis guizhouensis

Rhipidopsis lobulata

Rhipidopsis minutus

Rhipidopsis multifurcata

Rhipidopsis panii

Rhipidopsis shuichengensis

Rhizomopsis gemmifera

Samaropsis sp.

Schizoneura brevifolia

Schizoneura manchuriensis

Sigillaria sp.

Sphenophyllum sp.

Sphenophyllum koboense

Sphenophyllum sino-coreanum

Sphenophyllum speciosum

Sphenopteridium pseudogermanicum

Sphenopteris sp.

Sphenopteris tenuis

Squamocarpus papilioformis

Stigmaria sp.

Stigmaria ficoides

Stigmaria rugulosa

Strigillotheca fasciculata

Taeniopteris sp.

Taeniopteris crassinervis

Taeniopteris nystroemii

Taeniopteris rarinervis

Thallites sp.

Ullmannia bronnii

Ullmannia frumentaria

Wumengopteris crassirachis 


\section{China (Heilongjiang)}

\section{Permian (unspecified)}

Noeggerathiopsis mishanensis

\section{Upper Permian}

Cladophlebis xiaohinganensis

Comia major

Comia microphylla

Comia multinervis

Comia obese

Comia shenshuensis

Comia tenueaxis

Comia yichunensis

Compsopteris adzvensis

Compsopteris contractus

Compsopteris tchirkovae

Ginkgophyllum sp.

Ginkgophytopsis xinganensis

Iniopteris sibirica

Lepeophyllum trigonum

Lepidophyllum sp.

Lobatannularia heianensis

Lobatannularia multifolia

Nilsonia hongshanensis

Noeggerathiopsis angustifolia

Noeggerathiopsis insignis

Noeggerathiopsis synensis

Noeggerathiopsis tieliensis

Noeggerathiopsis xinganensis

Odontopteris xinganensis

Pecopteris anbangensis

Pecopteris cyathea

Pecopteris deducans

Pecopteris hongshanensis

Pecopteris tangwangheensis

Pecopteris yabei

Prynadaeopteris anthriscifolia

Pterophyllum slobodskiensis

Radicites sp.

Rhipidopsis hongshanensis

Rhipidopsis shifaensis

Rhipidopsis tangwangheensis

Rhipidopsis xinganensis

Schizoneura manchuriensis

Sphenophyllum sp.

Sphenopteris heilongjiangensis

Sphenopteris yichunensis

Supaia shenshuensis

Supaia tieliensis

Tychtopteris arunensis

Tychtopteris cuneata

Viatcheslavia sp.

Viatcheslavia vorcutensis

Xinganphyllum sp.

Xinganphyllum aequale

Xinganphyllum inaequale

Zamites kryshtofovichii

\section{Lower Permian}

Annularia longissima Callipteris angustata Callipteris biforma Callipteris confluens Callipteris congermana Callipteris heilongjiangensis Callipteris hongshanensis Callipteris jerunakovensis Callipteris obese Callipteris pseudoshenshuensis Callipteris shenshuensis Callipteris tangwangheensis Callipteris tatianaeana Callipteris zeilleri Crassinervia mishanensis Nephropsis cochlearis Nephropsis elegans Nephropsis rhomboidea Neuropteris daheshenensis Noeggerathiopsis aequalis Noeggerathiopsis batschatensis Noeggerathiopsis brevifolia Noeggerathiopsis derzavinii Noeggerathiopsis ensiformis Noeggerathiopsis kryshtofovichii Noeggerathiopsis latifolia

Noeggerathiopsis lianzhushanensis

Noeggerathiopsis obovata Noeggerathiopsis theodori Sphenopteris incrassata Zamiopteris glossopteroides Zamiopteris lanceolata Zamiopteris tailuganensis

\section{China (Henan)}

\section{Upper Permian}

\author{
Alethopteris shengi \\ Bergeria sp. \\ Chiropteris reniformis \\ Cladophlebis henanensis \\ Cladophlebis permica \\ Compsopteris imparis \\ Compsopteris wongii \\ Fascipteridium ellipticum \\ Gigantonoclea lagrelii \\ Gigantonoclea rosulata \\ Ginkgoites xinhuaensis \\ Henanophyllum palamifolium \\ Knorria sp. \\ Lepidodendron cervicisum \\ Lepidodendron polygonale \\ Lobatannularia ensifolia \\ Lobatannularia heianensis \\ Lobatannularia lingulata \\ Lobatannularia sinensis \\ Otofolium ovatum \\ Pseudorhipidopsis brevicaulis
}


Psygmophyllum multipartitum Sphenophyllum koboense Taeniopteris hunanensis

Taeniopteris taiyuanensis

Taeniopteris tingii

Tingia yichuanensis

\section{Lower Permian}

Alethopteris yiyangensis Annularia gracilescens Bothrodendron henanense Cathaysiopteris whitei Cladophlebis nystroemii Emplectopteridium alatum Emplectopteris triangularis Lepidodendron henanense Lepidodendron incertum Lepidodendron pingdingshanense Odontopteris henanensis Odontopteris orbicularis Odontopteris yiyangensis Pecopteris punicoides Plagiozamites oblongifolius Sphenopecopteris beaniata Sphenophyllum elagatiefolium Sphenophyllum gongxianense Sphenophyllum thonit Sphenophyllum verticillatum Sphenophyllum yiyangense Sphenopteris gongxianensis Sphenopteris tenuis

Tingiostachya $\mathrm{sp}$.

\section{China (Hunan)}

\section{Upper Permian}

Annularia hunanensis

Annularia mucronata

Calamites sp.

Cathaysiopteris sp.

Cordaites principalis

Gigantonoclea sp.

Gigantonaclea yabei

Gigantopteris nicotianaefolia

Rhipidopsis minor

Sphenobaiera biloba

\section{Lower Permian}

Sigillaria sp.

\section{China (Inner Mongolia)}

\section{Upper Permian}

Callipteris sp.

Callipteris shenshuensis

Comia sp.

Fa'scipteris densata
Fascipteris hallei

Pecopteris anthriscifolia

Pecopteris lativenosa

Pecopteris polymorpha

Pterophyllum daihoense

Sphenobaiera spirata

Sphenophyllum minor

Sphenophyllum spathulatum

Sphenopteris sp.

Sphenopteris firmata

Sphenopteris rotundata

\section{Lower Permian}

Alethopteris kaipingiana

Alethopteris norinii

Annularia mucronata

Annularia stellata

Calamites cistii

Cladophlebis manchurica

Cladophlebis yongwolensis

Emplectopteris neimongolensis

Emplectopteris sinensis

Emplectopteris triangularis

Lepidodendron carinum

Lepidodendron tachingshanense

Pecopteris anderssonii

Pecopteris arcuato

Pecopteris feminaeformis

Pecopteris lativenosa

Pecopteris orientalis

Pecopteris polymorpha

Pecopteris tuberculata

Pecopteris wongii

Pterophyllum diahoense

Rhacopteris bertrandii

Sphenobaiera spirata

Sphenophyllum kawasakil

Sphenophyllum minor

Sphenophyllum oblongifolium

Sphenophyllum scopulatum

Sphenophyllum spathulatum

Sphenopteridium pseudogermanicum

Sphenopteris firmata

Sphenopteris rotundata

Taeniopteris norinii

Taeniopteris tingil

Tingia hamaguchii

\section{China (Jiangsu)}

\section{Upper Permian}

Pecopteris nervosa

\section{China (Jilin)}

\section{Upper Permian}

Callipteris jerunakovensis

Crassinervia sp. 
Fascipteris kaishantunensis

Fascipteris robusta

Paraschizoneura sibirica

Pecopteris tumenjiangensis

Pecopteris yabei

Sphenophyllum koboense

Sphenophyllum speciosum

\section{Lower Permian}

Neuropteris dagangensis

Neuropteris daheshenensis

Sphenophyllum oblongifolium

Sphenophyllum thonii

Taeniopteris taiyuanensis

\section{China (Kansu)}

\section{Lower Permian}

Annularia sp.

Asterotheca sp.

Bowmanites sp.

Calamites sp.

Carpolithes sp.

Carpolithes galopinii

Chiropteris sp.

Cladophlebis sp.

Cladophlebis heishanensis

Conchophyllum sp.

Conchophyllum parvifolium

Cordaianthus sp.

Cordaicarpus sp.

Cordaites sp.

Corynepteris sp.

Corynepteris kansuensis

Corynepteris sternbergii

Crossotheca sp.

Equisetites sp.

Gansucladus parvifolia

Glossopteris sp.

Gondwanidium sp.

Huihuipuia reticulata

Linopteris sp.

Macrostachya sp.

Mariopteris crassipinnulata

Mariopteris yuemenensis

Mixoneura sp.

Nemejcopteris feminaeformis

Neurodontopteris sp.

Neuropteris sp.

Neuropteris pseudovata

Pecopteris sp.

Pecopteris saraepontana

Phylladoderma arberi

Phyllotheca sp.

Plagiozamites sp.

Plagiozamites oblongifolius

Psaliangium sp.

Rhacopteris sp.
Samaropsis sp.

Sphenophyllum sp.

Sphenophyllum emarginatum

Sphenopteris sp.

Sphenopteris damesii

Sphenopteris huihuipuensis

Stockmansia salviniaefolia

Sylvella sp.

Taeniopteris sp.

Trigonocarpum laguncula

Validopteris sp.

Walchia sp.

\section{China (Liaoning)}

\section{Upper Permian}

Callipteris pseudoshenshuensis

Callipteris shenshuensis

Callipteris tongxingensis

Cordaites principalis

Cladophlebis manchurica

Cladophlebis permica

Cladophlebis yongwolensis

Comia osinowskiensis

Comia tenueaxis

Comia yichunensis

Danaeites guangxingense

Fascipteris hallei

Fascipteris sinensis

Gigantonoclea microphylla

Gigantonoclea tieyingensis

Gigantonoclea unita

Lepeophyllum gemmatum

Lepeophyllum liaoningense

Lobatannularia heianensis

Lobatannularia lingulata

Neuropteridium tieyingensis

Pecopteris candolleana

Pterophyllum eratum

Schizoneura manchuriensis

Sphenophyllum koboense

Taeniopteris densissima

Taeniopteris guangxingensis

Taeniopteris szei

\section{Lower Permian}

Callipteridium tachingshanense

Cladophlebis manchurica

Cordaites yujiaensis

Gigantonoclea yujiaensis

Lepidodendron sp.

Linopteris sp.

Nilsonia huabeiensis

Odontopteris chui

Pecopteris candolleana

Pecopteris cyathea

Pecopteris densifolia 
Pecopteris sahnii

Pecopteris wongi

Sphenophyllum oblongifolium

Sphenophyllum juyiaense

Taeniopteris integra

Taeniopteris szei

Tingia carbonica

Tingia hamaguchii

Tingia partita

\section{China (Shanxi)}

\section{Permian (unspecified)}

Alethopteris norinii

Emplectopteris triangularis

Sagittapteris belemnopteroides

Sphenopteris tenuis

\section{Upper Permian}

Bicoemplectopteris hallei

Gigantonoclea lagrelii

Tricoemplectopteris taiyuanensis

\section{Lower Permian}

Cathaysiopteris whitet

Emplectopteris triangularis

Gigantonoclea lagrelii

\section{China (Sichuan)}

\section{Upper Permian}

Annularia pingloensis Carpolithes sp.

Compsopteris contracta

Cordaites principalis

Danaeites rigida

Gigantonoclea guizhouensis

Gigantopteris dictyophylloides

Gigantopteris nicotianaefolia

Lobatannularia ensifolia

Lobatannularia heianensis

Lobatannularia lingulata

Lobatannularia multifolia

Neuropteridium coreanicum

Paracalamites stenocostatus

Pecopteris orientalis

Pecopteris sahnii

Pecopteris tenuicostata

Psaronius hexagonus

Rhipidopsis panii

Samaropsis taiyuanensis

Schizoneura manchuriensis

Sphenobaiera tenuistriata

Stigmaria ficoides

Taeniopteris multinervis
Taeniopteris norinii

Taeniopteris taiyuanensis

\section{China (Xinjiang)}

\section{Upper Permian}

Comia cangfanggouensis Lepeophyllum ovatum Supaia serrata Tychtopteris densinervis Tychtopteris obesa

\section{China (Xizang)}

\section{Upper Permian}

Brongniartites sp.

Callipteris sp.

Callipteris sahnii

Compsopteris sp.

Dichotomopteris qubuensis

Dizeugotheca qubuensis

Ginkgophytopsis sp.

Glossopteris angustifolia

Glossopteris communis

Glossopteris indica

Iniopteris sp.

Peltaspermum sp.

Pursongia sp.

Raniganjia qubuensis

Sphenophyllum speciosum

Sylvella sp.

Syniopteris sp.

\section{China (Yunnan)}

\section{Upper Permian}

Abrotopteris guizhouensis Annularia pingloensis Annularia shirakii

Bifariusotheca qinglongensis Calamites sp.

Carpolithes glansiformis

Carpolithes minutus

Carpolithes punctatus

Carpolithes speculatus

Chansitheca kidstonii

Cladophlebis fuyuanensis

Compsopteris punctinervis

Cornucarpus sp.

Cladophlebis ozakii

Cladophlebis parapermica

Cladophlebis permica

Cladophlebis yunnanica

Discinites orientalis

Discinites crossothecoides

Fascipteris densata 
Fascipteris hallei

Fascipteris stena

Gigantonoclea guizhouensis

Gigantonoclea hallei

Gigantonoclea lagrelii

Gigantonoclea plumosa

Gigantopteris dictyophylloides

Gigantopteris paradoxa

Guizhoua gregalis

Lepidodendron acutangulum

Lepidodendron lepidophloides

Lepidodendron oculus-felis

Lepidodendron xuanweiense

Lepidostrobus acutisquamus

Linophyllum xuanweiense

Lobatannularia cathaysiana

Lobatannularia lingulata

Lobatannularia multifolia

Lopadiangium acmodontum

Lophotheca panxianensis

Neurophyllites pecopteroides

Neuropteridium guizhouense

Otofolium ovatum

Paracalamites stenocostatus

Pecopteris echinata

Pecopteris elegantula

Pecopteris fuyuanensis

Pecopteris guizhouensis

Pecopteris lingulata

Pecopteris longifolioides

Pecopteris marginata

Pecopteris qingyunensis

Pecopteris sahnii

Pelourdea contracta

Plagiozamites oblongifolius

Prionophyllopteris spiniformis

Pterophyllum eratum

Rajahia guizhouensis

Rajahia major

Rajahia minor

Rajahia mirabilis

Rajahia rigida

Rhabdocarpos olivaeformis

Rhipidopsis ginkgoides

Rhipidopsis lobulata

Rhipidopsis panii

Rhizomopsis gemmifera

Schizoneura brevifolia

Schizoneura manchuriensis

Sphenophyllum sp.

Sphenophyllum koboense

Sphenophyllum sino-coreanum

Squamocarpus papilioformis

Stigmaria ficoides

Strigillotheca fasciculata

Taeniopteris crassinervis

Taeniopteris rarinervis

Thallites sp.

Ullmannia bronnii

Ullmannia frumentaria
C.S.S.R. (Ceské Zemé - "Bohemia" -)

\author{
Lower Permian \\ Callipteris sp. (LPAutu) \\ Callipteris conferta (LPAutu)
}

\section{France (-)}

Permian (unspecified)

Lebachia sp.

\section{Lower Permian}

Callipteris conferta

Walchia piniformis

France (Allier)

Lower Permian

Dicranophyllum striatum (LPAutu)

France (Aveyron)

Lower Permian

Lebachia piniformis (LPAutu)

France (Hérault)

Lower Permian

Callipteris conferta

Lebachia piniformis (LPAutu)

Supaia thinnfeldioides (LPAutu?)

Trichopitys heteromorpha (LPAutu)

\section{France (Loire)}

\section{Lower Permian}

Dicranophyllum gallicum (LPAutu) Sphenopteris germanica (LPAutu)

\section{France (Saône-et-Loire)}

\section{Lower Permian}

Arthropitys bistriata

Biscalitheca dubius (LPAutu)

Callipteris conferta (LPAutu)

Dadoxylon sp. (LPAutu)

Dadoxylon rollei (LPAutu)

Dicranophyllum gallicum (LPAutu)

Etapteris lacattei

Palaeoxylon sp.

Poroxylon sp. 
Scleromedulloxylon varollense (LPAutu)

Taeniopteris doubingeri (LPAutu)

\section{France (Var)}

\section{Upper Permian}

Trichopitys gracilis

\section{Lower Permian}

Trichopitys gracilis (LPSaxo)

\section{France (Vosges)}

\section{Lower Permian}

Cyclopteris sp. (LPAutu)

Neuropteris neuropteroides (LPAutu)

Pecopteris polypodioides (LPAutu)

Poa-Cordaites sp. (LPAutu)

Taeniopteris multinervis (LPAutu)

\section{G.B. (Falkland Islands)}

\section{Permian (unspecified)}

Barakaroxylon lafoniense

\section{G.D.R. (-)}

\section{Lower Permian}

Psaronius sp.

Scolecopteris cyathea (LPRotl)

\section{G.D.R. (Dresden)}

\section{Lower Permian}

Asterotheca sternbergii (LPRotl) Callipteris flabellifera (LPRotl) Callipteris naumannii (LPRotl) Carpentiera frondosa (LPRotl) Cyclopteris rarinervia Dicranophyllum gallicum (LPRotl) Lebachia frondosa (LPRotl) Nemejcopteris feminaeformis (LPAutu) Pterispermostrobus wanderianus (LPRotl) Pterophyllum cotteanum (LPRotl) Weissites pinnatifidus (LPRotl)

\section{G.D.R. (Erfurt)}

\section{Lower Permian}

Alethopteris schneideri (LPAutu) Calamites gigas (LPRotl) Calamostachys dumasii (LPRotl)
Callipteris scheibei var. scheibei (LPAutu)

Lebachia piniformis (LPRotl)

Trichopitys heteromorpha (LPAutu)

Walchia germanica (LPAutu)

\section{G.D.R. (Gera)}

\section{Lower Permian}

Annularia carinata (LPRotl)

Annularia spicata (LPRotl)

Annularia stellata (LPRotl)

Aphlebia erdmannii (LPRotl)

Asterophyllites dumasii (LPRotl)

Asterophyllites equisetiformis fa. equisetiformis (LPAutu, LPRotl)

Asterotheca arborescens (LPAutu)

Asterotheca cyathea (LPAutu)

Asterotheca hemitelioides (LPAutu)

Calamariophyllum zeaeforme (LPRotl)

Calamites gigas (LPRotl)

Calamites multiramis (LPRotl)

Calamites suckowii (LPRotl)

Calamitina sp. (LPRotl)

Calamitopsis multiramis (LPAutu)

Calamostachys tuberculata (LPAutu, LPRot1)

Callipteris conferta (LPRot1)

Cardiocarpus gutbieri (LPAutu)

Dicksoniites pluckenetii (LPRotl)

Dicranophyllum gallicum (LPAutu)

Filicites aquilinus

Nemjcopteris feminaeformis (LPRotl)

Odontopteris osmundaeformis (LPAutu)

Odontopteris schlotheimii (LPRot])

Palaeostachya thuringiaca

Pecopteris monyi

Pecopteris polypodioides

Pecopteris potoniei

Psaronius sp. (LPRotl)

Schizostachys spiciformis (LPRotl)

Scolecopteris arborescens (LPRotl)

Scolecopteris candolleana (LPRotl)

Scolecopteris cyathea (LPRotl)

Scolecopteris densifolia (LPRotl)

Scolecopteris hemitelioides (LPRotl)

Scolecopteris oreopteridia (LPRotl)

Scolecopteris polymorpha (LPRotl)

Scolecopteris pseudobucklandii (LPRotl)

Sphenophyllum angustifolium (LPAutu)

Sphenophyllum thonii (LPAutu)

Taeniopteris jejunata (LPAutu)

Walchia filiciformia (LPAutu)

\section{G.D.R. (Halle)}

Permian (unspecified)

Sphenobaiera digitata 


\section{Upper Permian}

Callipteris martinsii (UPKupf)

Neocalamites mansfeldicus (UPKupf)

Sphenobaiera digitata (UPKupf)

Taeniopteris eckardtii (UPKupf)

Ullmannia frumentaria (UPKupf)

\section{Lower Permian}

Callipteris naumannii (LPAutu)

Plagiozamites sp. (LPAutu)

Plagiozamites planchardii (LPAutu)

\section{G.D.R. (KarI-Marx-Stadt)}

\section{Lower Permian}

Alethopteris schneideri (LPAutu, LPRotl) Alethopteris subelegans (LPRotl)

Ankyropteris scandens (LPRotl)

Annularia sp. (LPRotl)

Annularia carinata (LPRotl)

Annularia sphenophylloides (LPRotl)

Annularia spicata (LPRotl)

Annularia stellata (LPRotl)

Arthropitys bistriata (LPRotl)

Artisia approximata (LPRotl)

Asterochlaena laxa (LPRotl)

Asterophyllites equisetiformis (LPRotl)

Botryopteris burgkensis (LPRotl)

Calamitea bistriata (LPRotl)

Calamites sp. (LPRotl)

Calamites crassicaulis (LPRot1)

Calamites gigas (LPRotl)

Calamites infractus (LPRotl)

Calamites multiramis (LPRotl)

Calamites suckowii (LPRotl)

Calamodendron striatum (LPRotl)

Calamostachys sp. (LPRotl)

Calamostachys dumasii (LPRotl)

Calamostachys tuberculata (LPRotl)

Callipteridium gigas (LPRotl)

Callipteris conferta (LPRotl)

Callipteris naumannii (LPRotl)

Callipteris weberi (LPAutu)

Cardiocarpus elongatus (LPRotl)

Cardiocarpus reniforme (LPRotl)

Cordaianthus baccifer (LPRotl)

Cordaianthus gemmifer (LPRotl)

Cordaites sp. (LPRotl)

Cordaites foliolatus (LPRotl)

Cyclopteris sp. (LPRotl)

Dactylotheca elaverica (LPRotl)

Dadoxylon sp. (LPRotl)

Dicalamophyllum altendorfense (LPRotl)

Dicalamophyllum validum (LPRotl)

Dicksoniites pluckenetii (LPRotl)

Dicranophyllum sp. (LPRotl)

Dicranophyllum gallicum (LPRotl)

Ernestiodendron filiciformia (LPRotl)
Grammatopteris baldaufii (LPRotl)

Holcospermum sulcatum (LPRotl)

Lebachia sp. (LPRotl)

Macrostachya carinata (LPRotl)

Medullosa elegans (LPRotl)

Medullosa leuckartii (LPRotl)

Medullosa stellata (LPRotl, LPAutu)

Myeloxylon sp. (LPAutu)

Myeloxylon elegans (LPRotl)

Nemejcopteris feminaeformis (LPRotl)

Neuropteris cordata (LPRotl)

Neuropteris neuropteroides (LPRotl)

Neuropteris planchardii (LPRotl)

Noeggerathia zamitoides (LPRotl)

Odontopteris lingulata (LPRotl)

Palaeostachya superba

Pecopteris sp.

Pecopteris integra

Pecopteris mentiens

Peltostachys altendorfense (LPRotl)

Poa-Cordaites sp. (LPRotl)

Psaronius sp. (LPRotl, LPAutu)

Psaronius asterolithus

Psaronius infarctus (LPRotl)

Psaronius polyphyllus (LPRot1)

Psaronius simplex

Psaronius ungeri (LPRotl)

Psaronius weberi (LPRotl)

Pseudomariopteris ribeyronii (LPRotl)

Reticulopteris germari (LPRotl)

Scolecopteris arborescens (LPRotl)

Scolecopteris candolleana (LPRotl)

Scolecopteris densifolia (LPRotl)

Scolecopteris hemitelioides (LPRotl)

Scolecopteris lepidorhachis (LPRotl)

Scolecopteris permica (LPRotl)

Scolecopteris polypodioides (LPRotl)

Senftenbergia saxonica (LPRotl)

Sigillaria brardii (LPRotl)

Sphenophyllum sp. (LPRotl)

Sphenophyllum angustifolium (LPRotl)

Sphenophyllum oblongifolium (LPRotl)

Sphenophyllum thonii (LPRotl)

Sphenopteris guetzoldii (LPRotl)

Sphenopteris mathetii (LPRotl)

Taeniopteris abnormis (LPRotl)

Taeniopteris jejunata (LPRotl)

Trigonocarpum sp. (LPRotl)

Trigonocarpum noeggerathii (LPRotl)

Tubicaulis solenites (LPRotl)

Walchiopremnon sp. (LPRotl)

Weissites pinnatifidus (LPRotl)

Zygopteris primaria (LPRotl)

\section{G.D.R. (Leipzig)}

\section{Lower Permian}

Callipteris naumannii (LPRotl) Callipteris polymorpha (LPRotl) 
Lebachia goeppertiana (LPRotl) Odontopteris lingulata (LPRotl)

Pachytesta sp. (LPRotl)

Sphenopteris germanica (LPRotl)

\section{G.D.R. (Suhl)}

\section{Upper Permian}

Ullmannia lycopodioides (UPZech)

\section{Lower Permian}

Alethopteris grandinii (LPAutu) Alethopteris schneideri (LPAutu)

Annularia stellata (LPAutu)

Calamites gigas (LPRotl)

Callipterianthus arnhardtii (LPAutu)

Callipteris sp. (LPAutu)

Callipteris conferta (LPAutu)

Callipteris conferta var. supaioides (LPAutu)

Callipteris flabellifera (LPAutu)

Dicranophyllum gallicum (LPAutu, LPRotl)

Dicranophyllum hallei (LPAutu)

Lebachia piniformis (LPAutu)

Lilpopia crockensis (LPAutu)

Paracalamostachys heterospora (LPRotl)

Sphenopteris germanica (LPAutu)

\section{G.F.R. (Baden-Württemberg)}

\section{Lower Permian}

Callipteris conferta

Pterophyllum blechnoides (LPAutu)

\section{G.F.R. (Bayern)}

Permian (unspecified)

Myeloxylon $\mathrm{sp}$.

\section{Lower Permian}

Lesleya gimmii (LPAutu)

\section{G.F.R. (Hessen)}

Permian (unspecified)

Cupressites hulmannii

\section{Upper Permian}

Callipteris martinsii (UPZech, UPKupf)

Culmitzschia florinii (UPKupf)

Neocalamites mansfeldicus (UPKupf)

Pseudovoltzia sp. (UPKupf)

Pseudovoltzia liebeana (UPThur, UPKupf)
Sphenobaiera digitata (UPKupf)

Sphenopteris dichotoma (UPKupf)

Sphenopteris patens (UPKupf)

Taeniopteris eckardtii (UPKupf)

Ullmannia bronnii (UPKupf)

Ullmannia frumentaria (UPKupf)

\section{G.F.R. (Rheinland-Pfalz)}

Lower Permian

Callipteris conferta (LPRotl)

Cordaites sp. (LPRotl)

Odontopteris lingulata (LPAutu)

\section{G.F.R. (Rheinland-Westfalen)}

Upper Permian

Ullmannia bronnii (UPKupf)

\section{G.F.R. (Saarland)}

\section{Lower Permian}

Callipteris conferta (LPRotl, LPAutu)

Lebachia piniformis (LPRotl)

Lebachia speciosa (LPRotl)

Mesocalamites gigas (LPAutu)

Pecopteris gigantea

Taeniopteris multinervis (LPAutu)

Walchia piniformis (LPRot1)

\section{Greenland (East)}

Permian (unspecified)

Cordaites $\mathrm{sp}$.

\section{India (-)}

Permian (unspecified)

Denkania indica

Eretmonia ovoides

Glossopteris nimishea

Partha spathulata

Venustostrobus sp.

Upper Permian

Alethopteris sp. (UPBara Rani)

\section{Lower Permian}

Paratrizygia rhodesii (LPBara)

Lower Gondwana

Arberiella africana 
Glossopteris angustifolia

Glossopteris pantii (LGRani)

Glossopteris sastrii

Ottokaria sp.

Ottokaria bengalensis (LGKarh)

Vertebraria sp.

\section{India (Assam)}

\section{Lower Gondwana}

Glossopteris browniana

Glossopteris communis

Glossopteris parallela

Glossopteris stricta

\section{India (Bihar)}

\section{Upper Permian}

Buriadia sp. (UPKarh)

Glossopteris sp. (UPRani)

Glossopteris browniana (UPRani)

Glossopteris communis (UPRani)

Glossopteris conspicua (UPRani)

Glossopteris decipiens (UPRani)

Glossopteris divergens (UPRani)

Glossopteris fusa (UPRani)

Glossopteris leptoneura (UPRani)

Glossopteris nidpurensis (UPRani)

Glossopteris retifera (UPRani)

Glossopteris stricta (UPRani)

Palmatophyllites wardii (UPKarh)

Plumsteadia lanceolata (UPRani)

Rubidgea emarginata (UPKarh)

\section{Lower Permian}

Diphyllopteris verticillata (LPBara) Glossopteris decipiens (LPKarh)

Glossopteris longicaulis (LPKarh)

Neomariopteris barakarensis (LPBara)

Ottokaria biharensis (LPBara)

\section{Lower Gondwana}

Barakaroxylon jahriense (LGBara)

Barakaroxylon monocanalosum (LGBara)

Caulophyllites indica (LGKarh)

Cheirophyllum lacerata (LGKarh)

Dichotomopteris ovata (LGRani?)

Euryphyllum whittianum (LGKarh)

Gangamopteris angustifolia (LGKarh)

Gangamopteris clarkeana (LGKarh)

Gangamopteris cyclopteroides (LGKarh)

Glossopteris sp.

Glossopteris ampla (LGBara)

Glossopteris angusta (LGKarh)

Glossopteris angustifolia (LGRani, LGBara)

Glossopteris barakarensis (LGBara)
Glossopteris browniana (LGBara)

Glossopteris churiensis (LGBara)

Glossopteris communis (LGKarh, LGBara)

Glossopteris conspicua (LGBara)

Glossopteris damudica (LGKarh)

Glossopteris decipiens (LGKarh)

Glossopteris divergens (LGRani)

Glossopteris emarginata (LGBara)

Glossopteris fuchsii (LGBara)

Glossopteris giridihensis (LGKarh)

Glossopteris indica (LGBara, LGRani)

Glossopteris intermedia (LGBara, LGRani)

Glossopteris intermittens (LGBara)

Glossopteris karanpurensis (LGBara)

Glossopteris karharbariensis (LGKarh)

Glossopteris leptoneura (LGBara)

Glossopteris longicaulis (LGKarh)

Glossopteris nakkarea (LGBara)

Glossopteris nimishea (LGBara)

Glossopteris pandurata (LGBara, LGKarh)

Glossopteris pantii (LGRani)

Glossopteris raniganjensis (LGBara, LGBarM, LGRani)

Glossopteris recurva (LGKarh)

Glossopteris retifera (LGBara)

Glossopteris rewaensis (LGRani)

Glossopteris saksenae (LGBara)

Glossopteris stenoneura (LGBara)

Glossopteris stricta (LGKamt, LGRani)

Glossopteris syaldiensis (LGBara)

Glossopteris taeniensis (LGBara, LGKarh)

Glossopteris taenioides (LGKarh)

Glossopteris talchirensis (LGTalc)

Glossopteris tortuosa (LGBara)

Glossopteris varia (LGRani)

Glossopteris vulgaris (LGRani)

Glossopteris zeilleri (LGKarh)

Neomariopteris hughesii (LGKarh)

Noeggerathiopsis densinervis (LGKarh)

Ottokaria bengalensis (LGKarh)

Samaropsis ganjrensis (LGKarh)

Santhalea bansloiensis (LGRani?)

Stellotheca robusta

Vertebraria indica (LGBara, LGKarh)

\section{India (Jammu and Kashmir)}

Permian (unspecified)

Kashmiropteris meyenii

Kawizophyllum dunpathriensis

Pecopteris sp.

\section{India (Madhya Pradesh)}

Permian (unspecified)

Glossopteris indica

Upper Permian

Cistella sp. 
Glossopteris sp.

Glossopteris angustifolia

Glossopteris communis

Glossopteris feistmantelii (UPRani)

Glossopteris indica

Glossopteris leptoneura

Glossopteris longicaulis

Glossopteris retifera

Glossopteris stenoneura

Neomariopteris lobifolia

Schizoneura gondwanensis

Sphenopteris sp.

Vertebraria $\mathrm{sp}$.

\section{Lower Permian}

Glossopteris taenioides (LPKarh)

\section{Lower Gondwana}

Glossopteris bosei (LGRani) Glossopteris communis (LGKarh) Glossopteris feistmantelii (LGRani) Glossopteris indica (LGKarh) Glossopteris raniganjensis (LGRani) Glossopteris rewaensis (LGKarh)

Glossopteris saksenae (LGBara)

Glossopteris spatulata (LGKarh)

\section{India (Maharashtra)}

\section{Upper Permian}

Glossopteris stricta (UPRani)

Neomariopteris polymorpha

Rhipidopsis gonduanensis

Taxopitys indica (UPKamt)

\section{Lower Gondwana}

Araucarioxylon loharense Glossopteris indica (LGKamt)

Glossopteris raniganjensis (LGKamt)

\section{India (Orissa)}

\section{Permian (unspecified)}

Dictyopteridium sporiferum Eretmonia utkalensis

Glossotheca utkalensis

\section{Upper Permian}

Denkania indica (UPRani) Eretmonia hinjridaensis (UPRani) Eretmonia ovoides (UPRani) Eretmonia utkalensis (UPRani) Lidgettonia mucronata (UPRani) Neomariopteris khanii (UPKamt) Partha spathulata (UPRani)

\section{Lower Gondwana}

Glossopteris maculata (LGRani)

Glossotheca orissiana

Glossotheca utkalensis

Scutum sahnii (LGRani)

\section{India (Uttar Pradesh)}

\section{Lower Gondwana}

Glossopteris indica (LGKamt)

Glossopteris raniganjensis (LGKamt)

Glossopteris stricta (LGKamt)

Glossopteris taeniopteroides

\section{India (West Bengal)}

\section{Upper Permian}

Asansolia phegopteroides (UPRani) Belemnopteris pellucida (UPRani) Belemnopteris sagittifolia (UPRani) Cornuspermum pennatus (UPRani) Damudosorus searsolensis (UPRani) Dictyopteridium feistmantelii (UPRani) Dictyopteridium sporiferum (UPRani) Glossopteris angustifolia (UPRani) Glossopteris browniana (UPRani) Glossopteris clarkei (UPRani) Glossopteris communis (UPRani) Glossopteris conspicua (UPRani) Glossopteris contracta (UPRani) Glossopteris divergens (UPRani) Glossopteris formosa (UPRani) Glossopteris ghusikensis (UPRani) Glossopteris indica (UPRani) Glossopteris intermedia (UPRani) Glossopteris orbicularis (UPRani) Glossopteris retifera (UPRani) Glossopteris tenuinervis (UPRani) Jambadostrobus pretiosus (UPRani) Kendostrobus cylindricus (UPRani) Ottokaria raniganjensis (UPRani) Palaeospiroxylon heterocellularis (UPRanil Parasphenophyllum crenulatum (UPRani) Platycardia bengalensis (UPRani)

Scutum leslium (UPRani)

Senotheca sp. (UPRani)

Senotheca murulidihensis (UPRani)

Trithecopteris gondwanensis (UPRani)

Trizygia speciosa (UPRani)

Venustostrobus diademus (UPRani)

\section{Middle Permian}

Glossopteris damudica

\section{Lower Permian}

Glossopteris intermittens (LPBara) 


\section{Lower Gondwana}

Arberiella vulgaris (LGRani)

Dictyopteridium feistmantelii (LGRani)

Eretmonia sp. (LGRani)

Eretmonia emarginata (LGRani)

Glossopteris sp. (LGRani)

Glossopteris angustifolia (LGRani)

Glossopteris arberi (LGRani)

Glossopteris bosei (LGRani)

Glossopteris brongniartii (LGRani)

Glossopteris browniana

Glossopteris conspicua (LGRani)

Glossopteris cordiformis (LGRani)

Glossopteris damudica (LGBarM)

Glossopteris divergens (LGRani)

Glossopteris euryneura (LGRani)

Glossopteris formosa (LGRani)

Glossopteris gigas (LGRani)

Glossopteris gondwanensis (LGRani)

Glossopteris indica (LGRani)

Glossopteris intermedia (LGRani)

Glossopteris intermittens (LGBara)

Glossopteris lanceolata (LGRani)

Glossopteris maculata (LGRani)

Glossopteris major (LGRani)

Glossopteris mohudaensis (LGRani)

Glossopteris nakkarea (LGBara)

Glossopteris nautiyalii (LGRani)

Glossopteris nimishea (LGBara)

Glossopteris oldhamii (LGRani)

Glossopteris orbicularis (LGRani)

Glossopteris pantii (LGRani)

Glossopteris radiata (LGRani)

Glossopteris raniganjensis (LGRani)

Glossopteris retifera (LGRani)

Glossopteris retusa (LGRani)

Glossopteris rewaensis (LGRani)

Glossopteris rhabdotaenioides (LGRani)

Glossopteris sahnii (LGRani)

Glossopteris sastrii (LGRani)

Glossopteris searsolensis (LGRani)

Glossopteris spatulata (LGRani)

Glossopteris srivastavae (LGRani)

Glossopteris stenoneura (LGBara)

Glossopteris subtilis (LGRani)

Glossopteris taenioides (LGRani)

Glossopteris tenuifolia (LGRani)

Glossopteris tenuinervis (LGRani)

Glossopteris tortuosa (LGRani)

Glossotheca immanis (LGRani)

Jambadostrobus pretiosus (LGRani)

Plumsteadiostrobus sp. (LGRani)

Plumsteadiostrobus ellipticus (LGRani)

Pterygospermum raniganjense (LGRani)

Scirroma sp. (LGRani)

Scirroma angusta (LGRani)

Scirroma ventilebra (LGRani)

Venustostrobus diademus (LGRani)

Venustostrobus indicus (LGRani)

\section{Indonesia (Sumatra)}

\section{Lower Permian}

Gothanopteris bosschana

Palaeogoniopteris mengkarangensis

\section{Italy (Bolzano)}

\section{Upper Permian}

Masculostrobus sp.

Ullmannia sp.

Ullmannia bronnii

Ullmannia frumentaria

Voltzia sp.

Voltzia hungarica

\section{Italy (Brescia)}

\section{Lower Permian}

Callipteris nicklesii (LPSaxo)

Culmitzschia sp. (LPSaxo)

Lebachia hypnoides (LPSaxo)

Lepeophyllum sp. (LPSaxo)

Lesleya eckhardtii (LPSaxo)

Quadrocladus sp. (LPSaxo)

Sphenopteris interrupte-pinnata (LPSaxo)

Sphenopteris kukukiana (LPSaxo)

Sphenopteris patens (LPSaxo)

Sphenopteris suessii (LPSaxo)

Telangium sp. (LPSaxo)

Ullmannia frumentaria (LPSaxo)

Walchia geinitzii (LPSaxo)

Walchia germanica (LPSaxo)

Walchia stricta (LPSaxo)

Walchiostrobus sp. (LPSaxo)

\section{Japan (-)}

\section{Lower Permian}

Ginkgophyton maiyaense

\section{Laos (-)}

\section{Permian (unspecified)}

Bicoemplectopteris hallei

Cladophlebis ozakii

Pecopteris hemitelioides

Pterophyllum fromagetii

Sphenozamites sp.

\section{Madagascar (Sakamena)}

\author{
Upper Permian \\ Dadoxylon malaimbandense
}




\section{Madagascar (Sakoa Basin)}

\section{Lower Gondwana}

Cordaicarpus sp. (LGKarh-Bara?)

Cordaicarpus madagascariensis (LGKarh-Bara?)

Dolianitia madagascariensis (LGKarh-Bara?)

Elatra bella (LGKarh-Bara?)

Gangamopteris sp. (LGKarh-Bara?)

Gangamopteris cyclopteroides (LGTalc- Karh?)

Gangamopteris oblanceolata (LGTalc-Karh?)

Glossopteris sp. (LGKarh Bara?)

Glossopteris browniana (LGKarh-Bara?)

Glossopteris communis LGKarh-Bara?)

Glossopteris damudica (LGKarh-Bara?)

Glossopteris indica (LGKarh Bara?)

Lesleya sp. (LGTalc Karh?)

Noeggerathiopsis hislopi (LGKarh-Bara?)

Sakoarota polyangiata (LGKarh -Bara?)

Sphenophyllum sakoense (LGKarh-Bara?)

Sphenopteris sp. (LGKarh Bara?)

Taeniopteris punctifera (LGKarh-Bara?)

\section{Malaysia (-)}

\section{Permian (unspecified)}

Ptychocarpus malaianus

Rajahia linggiuensis

\section{Mongolia (-)}

\section{Upper Permian}

Bachtia sp.

Callipteris sp.

Callipteris altaica

Callipteris confluens

Callipteris jerunakovensis

Callipteris mongoliaensis

Callipteris orientalis

Callipteris sahnii

Carpolithes sp.

Cladophlebis sp.

Cladophlebis mongolica

Cladostrobus lutuginii

Comia sp.

Comia enisejevensis

Compsopteris sp.

Cordaicladus sp

Cordaites sp.

Cordaites adleri

Cordaites gracilentus

Cordaites kuznetskianus

Cordaites tologoiensis

Cordaites truncatus

Crassinervia sp.

Crassinervia ovifolia

Eichualdia sp.

Equisetum sp.

Ginkgoites sp
Glottophyllum sp.

Glottophyllum cuneatum

Glottophyllum karpovii

Iniopteris sp.

Laxostrobus sp.

Lepeophyllum sp.

Lepeophyllum actaeonelloides

Lepeophyllum belovoense

Niazonaria sp.

Pecopteris anthriscifolia

Pecopteris bobrovii

Pecopteris julii

Pecopteris laxifolia

Pecopteris leninskiensis

Pecopteris mucronata

Rhipidopsis palmata

Rufloria brevifolia

Rufloria delicata

Rufloria lepeophylloides

Rufloria magnifolia

Rufloria ulannurica

Samaropsis kureikaensis

Skokia elongata

Sphenopteris grabaui

Sphenopteris taiyuanensis

Taeniopteris sp.

Tchernovia sp.

Tungussocarpus tychtensis

Tychtopteris cuneata

Yavorskyia hebetata

Zamiopteris sp.

Zamiopteris dubia

\section{Lower Permian}

Annularia undulata

Bardocarpus tunguskaensis

Cordaites latifolius

Crassinervia oblongifolia

Rufloria sp.

Rufloria derzavinii

Rufloria kerulenica

Rufloria rasskasovae

Rufloria tuberculosa

Samaropsis sp.

Skokia elongato

Sphenopteris tunguscana

Vojnovskya sp.

Vojnouskya minima

\section{Mozambique (Zambesi Basin)}

Permian (unspecified)

Zalesskyoxylon zambesiensis

\section{Namibia (-)}

Permian (unspecified)

Paracalamites sp. 


\section{Upper Permian}

Brasilodendron africanum (U?P)

Brasilodendron pedroanum (U?P)

Lycopodiopsis costilatus (U?P)

Lycopodiopsis derbyi (U?P)

\section{Karroo}

Glossopteris sp.

Protophyllocladoxylon sp.

\section{New Zealand (South Island)}

Permian (unspecified)

Glossopteris sp.

$$
\text { Upper Permian }
$$

Glossopteris ampla (UPKaza)

\section{North Korea (Kaisen, Heian-Nando)}

\section{Lower Permian}

Bicoemplectopteridium longifolium

\section{North Korea (Tokusen)}

\section{Lower Permian}

Gigantopteris nicotianaefolia

\section{Poland (Promnik Basin) \\ Upper Permian}

Voltzia liebeana (UPZech)

\section{Rhodesia (-)}

Permian (unspecified)

Glossopteris sp.

\section{Saudi Arabia ('Unayzah)}

\section{Lower Permian}

Annularia stellata

Cordaites principalis

Marattiopsis sp.

Pecopteris sp.

Pecopteris hemitelioides

\section{South Africa (-)}

Permian (unspecified)

Glossopteris sp.

\section{Lower Gondwana}

Lycopodiopsis sp.

Lycopodiopsis costilatus

Lycopodiopsis derbyi

\section{South Africa (Cape Province)}

\section{Karroo}

Gangamopteris sp.

Gondwanidium sp.

Ecca

Glossopteris sp.

\section{South Africa (Natal)}

Permian (unspecified)

Arberiella sp.

Equisetum sp.

Eretmonia sp.

Lidgettonia sp.

Lidgettonia africana

\section{Upper Permian}

Arberiella sp.

Belemnopteris elongata

Dictyopteridium fabellatum

Dictyopteridium sporiferum

Eretmonia natalensis

Fetura natalensis

Lidgettonia africana

Mooia lidgettonioides

Phyllotheca australis

Plumsteadia sp.

Plumsteadia natalensis

Rigbya arberioides

Rusangea elegans

Shizoneura gondwanensis

Scopus confertus

Scopus didiscus

Scopus gibbosus

Scopus obscurus

Sphenophyllum speciosum

Sphenopteris sp.

Sphenopteris alata

\section{Karroo}

Dadoxylon arberi (UK)

South Africa (Orange Free State)

Permian (unspecified)

Glossopteris sp.

Karroo

Gondwanidium sp. 
Ecca

Tordoxylon steynspruitense

\section{South Africa (Transvaal)}

Permian (unspecified)

Glossopteris sp.

Glossopteris ampla

Glossopteris andreanskyi

Glossopteris brouniana

Glossopteris communis

Glossopteris cyclopteroides

Glossopteris damudica

Glossopteris divergens

Glossopteris indica

Glossopteris intermittens

Glossopteris kashmirensis

Glossopteris maccovi

Glossopteris major

Glossopteris obliqua

Glossopteris obovata

Glossopteris pseudocommunis

Glossopteris taeniopteroides

Glossopteris tortuosa

Gondwanidium sp.

Rubidgea sp.

\section{Lower Permian}

Cladophlebis nebbensis

Pecopteris plumsteadiae

Pecopteris singeri

Psygmophyllum kidstonii

Sphenophvllum thonii

\section{Lower Gondwana}

Lycopodiopsis sp.

Lycopodiopsis derbvi

\section{South Africa (Zululand)}

Permian (unspecified)

Osmundacaulis stumkei

\section{South Korea (Seizen)}

\section{Upper Permian}

Emplectopteridium alatum

\section{Spain (Gerona)}

\section{Lower Permian}

Ernestiodendron filiciformia (LPAutu)

Lebachia piniformis (LPAutu)

Ullmannia frumentaria (LPAutu)

Walchia germanica (LPAutu)

\section{Spain (Guadalajara)}

Lower Permian (all LPAutu)

Callipteris conferta

Callipteris raymondii

Odontopteris gimmii

Pecopteris pectinata

\section{Spain (Sevilla)}

Permian (unspecified)

Dadoxylon sp.

\section{Lower Permian}

Ginkgoites sp. (LPAutu?)

Ginkgophyllum sp. (LPAutu?)

Pecopteris lamuriana (LPAutu?)

Protoblechnum wongii (LPAutu?)

\section{Spain (Soria)}

\section{Lower Permian (all LPAutu)}

Callipteris conferta

Cathaysiopteris whitei

Dizeugotheca sp.

Equisetites elongatus

Gamophyllites sp.

Gigantonoclea lagrelit

Koretrophyllites crassinervis

Lebachia piniformis

Lepidodendron posthumii

Paracordaites sp.

Tingia carbonica

Umbellaphyllites annularioides

\section{Thailand (-)}

Permian (unspecified)

Ginkgophyton komalarjunii

\section{Turkey (Anatolia)}

\section{Upper Permian}

Bicoemplectopteris hallei

\section{U.S.A. (Colorado)}

Lower Permian

Sphenopteris germanica (LPAutu)

\section{U.S.A. (Kansas)}

Lower Permian

Callipteris flabellifera (LPAutu) 
Callipteris moorei (LPAutu)

Sphenopteris sellardsii

\section{U.S.A. (New Mexico)}

\section{Lower Permian}

Calamites sp.

Lepidodendron sp.

Millaya tularosana (LPWolf)

Sigillaria sp.

\section{U.S.A. (Pennsylvania)}

\section{Lower Permian}

Lescuropteris moorii (LPAutu)

\section{U.S.A. (Texas)}

Permian (unspecified)

Gigantopteris sp.

Lobatannularia $\mathrm{sp}$.

\section{Lower Permian}

Archaeocycas whitei Cycadospadix yochelsonii Gigantopteridium americanum Sandrewia texana

\section{U.S.A. (West Virginia)}

\section{Lower Permian}

Alethopteris grandini Alethopteris serlii Alethopteris virginiana Annularia asteris Annularia sphenophylloides Annularia stellata Asterophyllites equisetiformis Asterophyllites longifolius Calamites carinatus Callipteridium sp. Callipteridium gigas Callipteridium pteridium Callipteris conferta Crossotheca $\mathrm{sp.}$ Crossotheca crepinii Crossotheca sagittata Danaeites emersonii Dolerotheca pennsylvanicum Eremopteris crenulata Eremopteris missouriensis Eusphenopteris obtusiloba Eusphenopteris striata Holcospermum sp. Lepidodendron aculeatum Lepidodendron worthenii Lescuropteris moorii
Neuropteris fimbriata

Neuropteris ovata

Neuropteris scheuchzeri

Odontopteris aequalis

Odontopteris brardii

Pecopteris arborescens

Pecopteris candolleana

Pecopteris cyathea

Pecopteris feminaeformis

Pecopteris hemitelioides

Pecopteris lamuriana

Pecopteris polymorpha

Pecopteris unita

Pseudomariopteris cordato-ovata

Sigillaria brardii

Sphenophyllum angustifolium

Sphenophyllum emarginatum

Sphenophyllum majus

Sphenophyllum oblongifolium

Stigmaria ficoides

Trigonocarpum sp.

Walchia sp.

\section{U.S.S.R. (Kazakhstan)}

\section{Upper Permian}

Phylladoderma stenuifolia

\section{Lower Permian}

Marginopteris kasachstanica

Rufloria bakanasica

\section{U.S.S.R. (Kazakhstan - Dzhungariya -)}

\section{Upper Permian}

Chulakia alveolata

Chulakia pilifera

Dzungariella fucoides

Dzungariella kizilkainarica

\section{U.S.S.R. (Kemerovskaya Oblast}

\section{- Kuzbass - )}

\section{Upper Permian}

Cladophlebis licharevii

Cordaites torulosus

Ricciopsis parva

Rufloria marginata

Rufloria mira

Rufloria seljatitskii

Sparsistomites communis

\section{Lower Permian}

Jaganovia ziminkensis Koretrophyllites gracilis Koretrophyllites latifolius 


\section{U.S.S.R. (Komi A.S.S.R.)}

\section{Upper Permian}

Bachtia sp. (UPKaza)

Bajdaievia longiuscula (UPKaza)

Bajdaievia speciosa (UPKaza, UPTata)

Intia falciformis (UPTata)

Kosjunia polyedra (UPKaza)

Rhaphidopteris praecursoria (UPKaza)

Syrjagia lituata (UPKaza)

Uskatia dentata (UPKaza)

Vorcutannularia laevis (UPTata, UPKaza)

\section{Lower Permian}

Bachtia sp. (LPUfim)

Bajdaievia speciosa (LPUfim)

Intia falciformis (LPUfim)

Intia variabilis (LPUfim)

Intia vermicularis (LPUfim)

Intia vicaria (LPUfim)

Junjagia glottophylla (LPUfim)

Kosjunia polyedra (LPUfim)

Kosjunia retusa (LPUfim)

Protosphagnum nervatum (LPUfim)

Salairia confinis (LPUfim)

Salairia singularis (LPUfim)

Syrjagia rectiserialis (LPUfim)

Uskatia dentata (LPUfim)

Uskatia vicina (LPUfim)

Vorcutannularia plicata (LPUfim)

\section{U.S.S.R. (Komi A.S.S.R. - Pechora Basin-)}

\section{Upper Permian}

Cardiolepis piniformis

Cladophlebis gorskii

Nucicarpus piniformis

Prynadaeopteris minuta

Prynadaeopteris rhomboidea

Slivkovia petschorensis

Sporophyllites petschorensis

\section{Lower Permian}

Vojnouskya paradoxa

\section{U.S.S.R. (Krasnoyarskiy Kray \\ - Tungusk Basin -)}

\section{Upper Permian}

Peltaspermum sp.

Rufloria ilimpejica

Rufloria tomentella

Tatarina lobata

Tatarina sadovnikovii

Umbellaphyllites nacumilensis

\section{Lower Permian}

Cardiocarpus cardiformis Cordaicarpus trapezoideus

Ricciopsis anakitensis

\section{U.S.S.R. (Primorskiy Kray)}

\section{Upper Permian}

Angaropteridium tyrganicum Asterotheca primorskiensis Cladophlebis melnikovii

Cordaites sp.

Cordaites buragoi

Cordaites primorskiensis

Crassinervia pentagonata

Gangamopteris sp.

Gangamopteris cyclopteroides

Gangamopteris pacifica

Gangamopteris ussuriensis

Ginkgophyton giganteum

Glossopteris sp.

Glossopteris indica

Glossopteris orientalis

Glossopteris tunguscana

Marattiopsis orientalis

Palaeovittaria sp.

Pecopteris dissimilaris

Pecopteris niamdensis fa. makuchinae Phylladoderma sp.

Psygmophyllum ussuriense

Rufloria derzavinii

Rufloria sublanceolata

Rufloria vassilyevii

Sphenobaiera macrofolia

Sphenozamites permicus

Taeniopteris sp.

Taeniopteris primorjensis

Taeniopteris sinegorkiensis

Zamiopteris dubia

Zamiopteris gorelovae

\section{Lower Permian}

Angaridium sp.

Angaropteridium sp.

Annularia tenuifolia

Annularia planifolia fa. crassinervia

Cardioneura pulchra

Cardioneura tebenjkovit

Cordaites sp.

Cordaites latifolius

Cordaites primorskiensis

Crassinervia sp.

Crassinervia grammii

Crassinervia neuburgiana

Crassinervia pentagonata

Crassinervia strelokensis

Crassinervia tunguscana

Gaussia scutellata 
Lepeophyllum trigonum

Nephropsis sp.

Nephropsis acuminata

Nephropsis asiatica

Nephropsis integerrima

Nephropsis lampadiformis

Nephropsis pospeloviensis

Nephropsis pseudosimilis

Paracalamites crassus

Paracalamites decoratus

Prynadaeopteris tunguscana

Rufloria sp.

Rufloria derzavinii

Rufloria mirabilis

Rufloria recta

Rufloria sublanceolata

Rufloria theodorii

Rufloria ussurica

Sphenophyllum sp.

Sphenophyllum meyenii

Sphenophyllum osipoviense

Vojnovskya elegans

Vojnouskya pacifica

Zamiopteris sp.

Zamiopteris daubichites

Zamiopteris glossopteroides

Zamiopteris gorelovae

Zamiopteris lanceolata

Zamiopteris rotunda

Zamiopteris schmalhausenii

Zamiopteris subglossopteroides

\section{U.S.S.R. (Vologda - Aristovo - )}

\section{Upper Permian}

Peltaspermum sp. (UPTata)

Peltaspermum buevichae (UPTata)

Phylladoderma aequalis (UPTata)

Quadrocladus dvinensis (UPTata)

Sashinia aristovensis (UPTata)

Tatarina conspicua (UPTata)

Tatarina pinnata (UPTata)

Tatarina sinuosa

Tatarina verrucosa

\section{U.S.S.R. (West Ural)}

Upper Permian

Sphenopteris interrupte-pinnata (UPKupf)

\section{U.S.S.R. (Yakutskaya A.S.S.R. - Verkhoyansk -)}

\section{Upper Permian}

Cordaites gracilentus

Cordaites minax

Laxostrobus sp.

Phyllotheca turnaensis

\section{Acknowledgements}

Apart from the contributors to the IFPM we would like to thank the librarians who assisted greatly in the realization of this volume: Mr. J.G. de Bruijn (Teyler Stichting Haarlem); Mrs. M.C. Draisma-van den Houten, Mr. J.C. Jansen, Mr. M.W.J.M. Stelling and Mrs. S. van Ingen-van Vliet (Inst. Aardwetenschappen Utrecht); Miss A. Lum (British Museum Nat. Hist. London); Mr. F.J.R. Öry Bertha and Mr. H. Kingma Boltjes (Rijksmus. Geol. Miner. Leiden); Mr. H.J. van den Berg (Lab. Palaeobot. Palynol. Utrecht); Mr. P.A. van den Berg (Inst. Syst. Plantkunde Utrecht) and Mrs. K. Willems-Dreezen (Rijks Geol. Dienst Heerlen).

\section{References}

Agashe, S.N. and Gowda, P.R.V., 1978. Anatomical study of a gymnospermous wood from Lower Gondwana strata of Maharashtra. Phytomorphology, 28(3): 269-275.

Alvarez-Ramis, C. and Doubinger, J., 1976. Sphénopteridées du bassin houiller de Saint-Etienne (Loire, France). C.R. 101ème Congr. Natl. Soc. Savantes, Sect. Sci., 1: 23-27.

Anderson, A.M. and McLachlan, I.R., 1976. The plant record of the Dwyka and Ecca Series (Permian) of the south-western half of the Great Karroo Basin, South Africa. Palaeontol. Afr., 19: 31-42.

Andreis, R.R., Cazzulo-Klepzig, M., Guerra-Sommer, M. and Zimmermann, L., 1980. Consideraçóes sobre um affloramento fossilífero do Grupo Itararé: Fazenda Goulart, Francisquinho, Município de São Jeronimo, RS. Bol. Inst. Geocienc. Univ. São Paulo, 11: 85-97 (with English summary).

Andrews, H.N., 1980. The Fossil Hunters: In Search of Ancient Plants. Cornell Univ. Press, Ithaca, N.Y., $421 \mathrm{pp}$.

Appert, O., 1977. Die Glossopteris-Flora der Sakoa in Südwest-Madagascar. Palaeontographica, B, 162(1/3): 1-50 (with French and English summaries).

Arrondo, O.G. and Petriella, B., 1979. The genus Lycopodiopsis (Lycopodiopsidaceae, Lepidodendrales) of the Middle Carboniferous-Lower Permian of Argentina. Ameghiniana, 16(1/2): 121-134 (in Spanish with English summary).

Asama, K., 1976. Gigantopteris flora in Southeast Asia and its phytopaleogeographic significance. Contrib. Geol. Palaeontol. Southeast Asia, 175: 191-207.

Asama, K., 1979. Evolution and phylogeny of vascular plants. Ann. Rep. Inst. Evol. Biol., 4/5: 3-48 (in Japanese).

Asama, K., 1980. Similarities in the patterns of macroevolution of vascular plants and vertebrates. In: Professor Saburo Kanno Mem. Vol. Tsukuba Univ., pp. 415-434. 
Azcuy, C.L., Cesari, S. and Longobucco, M., 1980. Nueva Licopsida fertil del Bajo de Veliz, San Luis, Bol. IGCP Project Upper Paleozoic of South America, Project 73/1/42-3, 4 pp.

Babin, C., 1980. Elements of Paleontology. Wiley, New York, N.Y., pp.168-195.

Balasundaram, M.S., Sastry, M.V.A., Shah, S.C. and Gopal Singh, 1976. Glossopteris flora in India. Rec. Geol. Surv. India, 107(2): 91117.

Banerjee, M., 1978a. Genus Glossopteris Brongniart and its stratigraphic significance in the paleozoics of India.1. A revisional study of some species of the genus Glossopteris. Bull. Bot. Soc. India, 32(1/2): 81-125.

Banerjee, M., 1978b. Glossopteridian fructifications.2. On the revision of Ottokaria Zeiller and the occurrence of $O$. raniganjensis Banerjee from the Raniganj Formation (Upper Permian) of India. Indian J. Earth Sci., 5(2): 129140.

Banerjee, M., 1979. Seed-bearing glossopteridean fertile organs. Proc. 4th Int. Gondwana Symp., Calcutta, 1977 1: $122 \cdots 132$.

Banerjee, M., Mitra, P. and Chakravorty, D.K., 1979. Occurrence of Lower Gondwana rocks in Western Garo Hills, India. Proc. 4th Int. Gondwana Symp., Calcutta, 1977, 1: 7179 .

Banerji, J., Maheshwari, H.K. and Bose, M.N., 1976, Some plant fossils from the Gopad River section near Nidpur, Sidhi district, Madhya Pradesh. Palaeobotanist, 23(1): 59.71

Bariand, N. and Pajaud, D., 1978. Fossilien. Versteinertes Leben aus Jahrmillionen. Franckh'sche Verlagshand lung, Stuttgart, $106 \mathrm{pp}$.

Barthel, M., 1976a. Alethopteris schneideri Sterzel -... eine stratigraphisch wichtige Form des Autunien in Mittel europa. Z. Geol. Wiss., 6: 881 889 (with Russian and English summaries).

Barthel, M., 1976b. Ergebnisse der floristischen Neubear beitung des Rotliegenden in Sachsen. Z. Geol. Wiss., 4(6): 921933 (with Russian and English summaries).

Barthel, M., 1977. Die Gattung Dicranophyllum Gr. Eury in den varistischen Innensenken der DDR. Hallesches Jahrb. Geowiss., 2: 73-86 (with English and Russian summaries).

Barthel, M., 1980a. Calamiten aus dem Oberkarbon und Rotliegenden des Thüringer Waldes. In: W. Vent (Editor), 100 Jahre Arboretum (1879-1979). Akademie-Ver lag, Berlin; pp.237-258 (with English summary).

Barthel, 1980b. Pecopteris (Scolecopteris) Arten aus dem Rotliegenden von Manebach in Thüringen. Wiss. Z Humboldt-Univ. Berlin. Math.-Naturwiss. Reihe, 29(3): 351-366 (with Russian, English and French summaries)

Barthel, M., 1980c. Pecopteris-Arten von E.F. von Schlot. heim aus Typuslokalitäten in der DDR. Schriftenr. Geol. Wiss., 16: 274--304 (with French, English and Russian summaries).

Barthel, M. and Fischer, H., 1980. Ein Psaronius-Fund aus pleistozänen Gera-Schottern. Fundgrube, 26(2): 63 .

Barthel, M., Götzelt, V. and Urban, G., 1976. Die Rotliegendflora Sachsens. Abh. Staatl. Mus. Mineral. Geol
Dresden, 24: $287 \mathrm{pp}$. (with English and Russian summaries).

Barthel, M. and Haubold, H., 1980. Zur Gattung Callipteris Brongniart.1. Die Ausbildung von Callipteris conferta (Sternberg) Brongniart im mitteleuropäischen Rotliegenden. Schriftenr. Geol. Wiss., 16: 49 105 (with French, English and Russian summaries).

Baxter, R.W., 1978. Sphenopteris sellardsii, a pteridosperm from the Permian of Kansas. Univ. Kans. Paleontol. Contrib. Pap., 94: 17

Belot, V.R., 1978. Guide des Minéraux, Coquillages et Fossiles: où les trouver en France, comment les Connaître et les Collectionner. Horay. Paris, $222 \mathrm{pp}$

Benecke, A.K., 1976. Several new forms of Glossopteris fructifications from the Beaufort Daptocephalus-zone (Upper Permian) of Natal. South Africa. Palaeontol. Afr., 19: 97-125.

Bernardes de Oliveira. M.E.C., 1978. Fructificaçòes de pteridospermófitas eogondvánicas da "Gamada Irapuá". Formação Rio Bonito, nos arredores de Criciúma, SC. An. 30 Congr. Bras. Geol, 2: 986-1001 (with English summary).

Bernardes de Oliveira, M.E.C., 1980. Nouveau genre néomarioptéridien fertile de la flore à Glossopteris du Bassin de Paraná au Brésil. Bol. Inst. Geocienc. Univ. São Paulo, 11: 113119.

Bernardes de Oliveira, M.E.C. and Da Silva Pontes, C.E., 1977. Algumas observaçòes sobre as Cordaitófitas da Formação Rio Bonita, Grupo Tubarão, Bacia do Paraná, Brasil. Actas Primer Congr. Geol. Chileno, 3: 21-81 (with Spanish summary).

Boersma, M., 1980. Problemen, mogelijkheden en doelstellingen van het pre-Tertiaire macropalaeobotanisch onderzoek. Grondboor Hamer, 34(4): 114-120 (with English summary).

Bohlin, B., 1976. Four localities with Late Palaeozoic plants in the chinese provinces Kansu and Kokonor. Rep. Sci. Exped. NW Prov. China, 53, 167 pp.

Bohlin, B., 1978. Pseudolycopsids from the Rocky Mountains of Canada. Bol. Inst. Geocienc. Univ. São Paulo, 9: $133-135$.

Bohlin, B., 1980. The Pseudolycopsids and the Glossopteris problem. A supplement to the author's Pseudolycopsids from the Rocky Mountains of Canada (1979). Privately printed, distributed by the Univ. Library Uppsala, 9 pp.

Bold, H.C., Alexopoulos, C.J. and Delevoryas, T., 1980. Morphology of Plants and Fungi. Harper and Row, New York, N.Y., $819 \mathrm{pp}$.

Bose. M.N., Banerji, J. and Maithy, P.K., 1977. Some fossil plant remains from Ramkola-Tatapani coalfield, Madhya Pradesh. Palaeobotanist, 24(2): 108117

Broutin. J., 1977. Nouvelles données sur la flore des bassins autuno-stéphaniens des environs de Guadalcanal (Province de Séville, Espagne). Cuad. Geol. Ibér., 4: 91-98.

Broutin, J., 1978. Dadoxylon (Cordaioxylon) sp. Grand' Eury, 1877. Note préliminaire sur les bois fossiles de Permien de Rio Viar (Province de Séville, Espagne). C.R. 103ème Congr. Natl. Soc. Savantes, Sect. Sci., 2: 47 52 .

Brown, J.T., 1977. Evidence for epidogenetic growth in Paracalamites from southwest Africa. S. Afr. J. Sci.. 73(8): $235 \cdot 237$. 
Burago, V.I., 1976. The floristic links between eastern and western parts of Angarida in the Permian. Paleontol. Zh., 1976(1): 94-103 (in Russian).

Burago, V.I., 1977. Elements of the Mesozoic flora in the Late Permian flora of southern Primor'ye. In: V.A. Krasilov (Editor), Palaeobotany of the Far East. Tr. Biol.-Pochv. Inst. Dal'nevost. Nauch. Tsentr Akad. Nauk S.S.S.R., 45-51 (in Russian).

Burago, V.I., 1978. Taeniopteris from Permian sediments in southern Primor'ye. Paleontol. Zh., 1978(1): 127-137 (in Russian).

Burago, V.I., 1979. Early Permian flora of the Daubikhinsk zone. Tr. Biol.-Pochv. Inst. Dal'nevost. Nauch. Tsentr Akad. Nauk S.S.S.R., 53(156): 49-67 (in Russian).

Burago, V.I., 1980a. New Late Permian ferns from southern Primor'ye. In: I.N. Srebrodol'skaya, G.A. Stukalina, A.F. Abushik and N.N. Bobkova (Editors), New species of ancient plants and invertebrates in the U.S.S.R.: Plants. Tr. Vses. Nauchno-Issled. Geol. Inst. Leningrad, 204: 38-40 (in Russian).

Burago, V.I., 1980b. New fern species from the Late Permian of southern Primor'ye. In: I.N. Srebrodol'skaya, G.A. Stukalina, A.F. Abushik and N.N. Bobkova (Editors), New species of ancient plants and invertebrates in the U.S.S.R.: Plants. Tr. Vses. Nauchno.-Issled. Geol. Inst. Leningrad, 204: 51-54 (in Russian).

Cazzulo-Klepzig, M., 1978. Estudo da tafoflórula do Membro Morro Pelado na sua localidade-tipo. Pesquisas, Inst. Geocienc. U.F.R.G.S., Porto Allegre, 11: 225-303 (with English summary).

Cazzulo-Klepzig, M., Guerra-Sommer, M. and Bossi, G.E., 1980a. Revisão fitoestratigráfica do Grupo Itararé no Rio Grande do Sul.1. Acapamento Velho, Cambaí Grande, Budó e Morro Papaléo. Bol. Inst. Geocienc. Univ. São Paulo, 11: 55 -76 (with English summary).

Cazzulo-Klepzig, M., Guerra-Sommer, M. and MarquesToigo, M., 1980b. Estudo macro e microflorístico do Grupo Itararé (Bacia de Paraná), Rio Grande do Sul. An. 31 Congr. Bras. Geol., Balneário de Camboriú, SC, 5: 3027-3040 (with English summary).

Chaloner, W.G., Leistikow, K.U. and Hill, A., 1979. Brasilodendron gen. nov. and B. pedroanum (Carruth. ers) comb.nov., a Permian lycopod from Brazil. Rev. Palaeobot. Palynol., 28(2): 117-136.

Chandra, S. and Surange, K.R., 1976. Cuticular studies of the reproductive organs of Glossopteris.1. Dictyopteridium feistmanteli sp.nov. attached on Glossopteris tenuinervis. Palaeontographica, B, 156(4/6): 87-102.

Chandra, S. and Surange, K.R., 1977a. Cuticular studies of the reproductive organs of Glossopteris.2. Cistella type fructification Plumsteadiostrobus ellipticus gen. et sp.nov. attached on Glossopteris taenioides Feistmantel. Palaeobotanist, 23(3): 161-175.

Chandra, S. and Surange, K.R., 1977b. Cuticular studies on the reproductive organs of Glossopteris.4. Venustostrobus indicus sp.nov. Palaeobotanist, 24(3): 149-160.

Chandra, S. and Surange, K.R., 1977c. Fertile bracts and scales of Glossopteris fructifications from the Lower Gondwana of India. Palaeobotanist, 24(3): 195-201.

Chandra, S. and Surange, K.R., 1977d. Some scale leaves and sporangia from the Raniganj Coalfield, India. Palaeobotanist, 24(3): 245-253.

Chandra, S. and Surange, K.R., 1977e. Cuticular studies of the reproductive organs of Glossopteris.3. Two new female fructifications - Jambadostrobus and Venustostrobus - borne on Glossopteris leaves. Palaeontographica, B, 164(4/6): 127-152.

Chandra, S. and Surange, K.R., 1979. Revision of the Indian species of Glossopteris. Monogr. Birbal Sahni Inst. Palaeobot. Lucknow, 2: $291 \mathrm{pp}$.

Chen, Y., Duan, S., Yang, X. and Guo, S., 1978. Plants. In: Palaeontological Atlas of Southwest China. Szechuan Vol.2. Geol. Publ. House, Beijing, pp.460-537 (in Chinese).

Chitnis, S.R. and Vagyani, B.A., 1979. Additions to the Glossopteris flora from the Kamthi Beds near Satnavari, district Nagpur (M.S.). Geophytology, 9(1/2): 62-64.

Correa da Silva, Z.C., 1978. Observaçōes sobre o Grupo Tubarão no Rio Grande do Sul com especial destaque á estratigrafia da Formação Itararé. Pesqui. Inst. Geocienc. U.F.R.G.S., Porto Allegre, 9: 9-61 (with English summary).

Correa da Silva, Z.C. and Arrondo, O.G., 1977. Tafoflora Permiana de Mariana Pimentel, município de Guaíba, Rio Grande do Sul, Brasil. Pesqui. Inst. Geocienc. U.F.R.G.S., Porto Allegre, 7: 27-44 (with English summary).

Craddock, C., 1978. Antarctica and Gondwanaland. In: M.A. McWhinnie (Editor), Polar Research: to the Present, and the Future. Westview Press, Boulder, Colo., 7: 63-96.

Daber, R., 1976. Entwicklungsgeschichte der Pflanzen. In: H. Böhme, R. Hagemann and R. Löther (Editors), Beiträge zur Genetik und Abstammungslehre. Volk und Wissen, Berlin, pp.471-493.

Daber, R., 1980a. Zum Problem der Gabelwedelformen des Karbons und Perms - eine Ubersicht. Schriftenr. Geol. Wiss., 16: 15-18 (with French, English and Russian summaries).

Daber, R., 1980b. 280 Millionen Jahre Ginkgo-Belegstücke zur Geschichte des Taxons Ginkgo L. aus den Sammlungen und Anlagen der Humboldt-Universität. In: W. Vent (Editor), 100 Jahre Arboretum Berlin (1879-1979). Akademie-Verlag, Berlin, pp.259-279 (with English summary).

Daber, R. and Helms, J., 1976. Mein kleines Fossilienbuch. Umschau, Frankfurt/M., 96 pp.

Daber, R. and Helms, J., 1978. Das große Fossilienbuch. Neumann-Neudamm, Melsungen, 264 pp.

De la Peña, J.A., Fonolla, F., Ramos, J.L. and Marfil, R., 1977. Identificacion del Autuniense en la rama aragonesa de la Cordillera Iberica (Provincia de Soria). Cuad. Geol. Iber., 4: 124-134 (with English and French summaries).

Delevoryas, T., 1979. Gymnosperms. In: R.W. Fairbridge and D. Jablonski (Editors), The Encyclopedia of Paleon tology, In: Encyclopedia of Earth Sciences. Dowden, Hutchinson and Ross, Stroudsburg, Pa., 7: pp.364-373.

Denizot, G., 1976. Atlas des Fossiles.1. Fossiles Primaires et Triasiques. Boubée, Paris, 6th ed., 87 pp. 
Dilcher, D.L., 1979. Early angiosperm reproduction: an introductory report. Rev. Palaeobot. Palynol., 27(3/4): 291-328.

Dobruskina, I.A., 1980. Position stratigraphique des couches floristiques du Trias en Eurasie. Tr. Geol. Inst. Akad. Nauk S.S.S.R., 346: 1-162 (in Russian).

Dohms, M.H., 1976. Nova madeira de gimnosperma do Permiano do Rio Grande do Sul. Rev. Bras. Geocienc., 6(3): 164-181 (with English summary).

Dolianiti, E., 1977. Chiropteris reniformis Kawasaki no Gondwana do Sul do Brasil. Perqui. Inst. Geocienc. U.F.R.G.S., Porto Allegre, 7: 23-26.

Doubinger, J. and Alvarez-Ramis, C., 1979. Note sur la flore autuno-stéphanienne d'Albé (Vosges moyennes, France) C.R. 104ème Congr. Natl. Soc. Savantes, Sect. Sci., 1: $133-139$.

Doubinger, J. and Heyler, D., 1976. Nouveaux fossiles dans le Permien français. Bull. Soc. Géol. Fr., Sér. 7. 17(6): $1176-1180$.

Doubinger, J., Robert, J.F. and Broutin, J., 1978. Données complémentaires sur la flore permo-carbonifère de Surroca-Ogassa (Province de Gérone, Espagne). C.R. 103ème Congr. Natl. Soc. Savantes, Paléobot., 2: 39 45.

Durante, M.V., 1976. The Carboniferous and Permian stratigraphy of Mongolia on the basis of palaeobotanical data. Trans. Sov. Mong. Sci. Res. Geol. Exped., 19 279 pp. (in Russian).

Durante, M.V., 1980. On the correlation of the Late Permian flora of Nan'shan with contemporary Angara floras. Paleontol. Zh., 1980(1): 125-135 (in Russian).

Durante, M.V. and Biterman, I.M., 1978. An analysis of floral data in connection with questions on the Permian-Triassic boundary in the western Verkhoyansk Range. In: L.I. Popeko (Editor), The Upper Paleozoic of northeastern Asia. Izd. Akad. Nauk Dal'nevost. Nauchn. Tsentr: 76-89 (in Russian).

El-Khayal, A.A., Chaloner, W.G. and Hill, C.R., 1980. Palaeozoic plants from Saudi Arabia. Nature, 285(5759): 33.34 .

Erasmus, T., 1976. On the anatomy of Dadoxylon arberi Seward with some remarks on the phylogenetical tendencies of its tracheid pits. Palaeontol. Afr., 19: $127-133$.

Escher, A. and Watt, W.S., 1976. Geology of Greenland. Geol. Surv. Greenland, Copenhagen, 603 pp.

Fefilova, L.A., 1978a. Sporophyllites from Upper Permian sediments in northern Priural and its taxonomic position. Tr. Inst. Geol. Komi Fil. Akad. Nauk S.S.S.R., 25: 29- 41 (in Russian).

Fefilova, L.A., 1978b. Permian Mosses from the Permian of the European Part of northern U.S.S.R. Nauka, Leningrad, $120 \mathrm{pp}$. (in Russian).

Feng, S., Chen, G., Xi, Y. and Zhang, C., 1977. Plants. In: Fossil Atlas of Central-South China, 2. Geol. Publ. House, Beijing, Hupei Inst. Geol. Sci., pp.622-674 (in Chinese).

Fischer, J.C. and Gayrard-Valy, Y., 1976. Fossiles de tous les Temps. Editions Pacifique, Papeete, 199 pp.

Fischer, J.-C. and Gayrard-Valy, Y., 1977. Je découvre les Fossiles. Leson, Paris, 137 pp.
Fittipaldi, F.C. and Rösler, O., 1978. Paranocladus? fallax (Conifera) estudos cuticulares. Bol. Inst. Geocienc. Univ. São Paulo, 9: 109-113 (with English summary).

Galtier, J., 1978. Prếcisions sur "Zygopteris lacattei" et "Botryopteris dubius", fougères très rares de l'Autunien d'Autun. Bull. Soc. Hist. Nat. Autun, 88: 18-24.

Gillespie, W.H., Glendenning, J.A. and Pfefferkorn, H.W., 1978. Plant Fossils of West Virginia and Adjacent Areas. State W. Va, Educ. Ser., ED-3A, W. Va. Geol. Econ. Surv., Morgantown, W.Va., 172 pp.

Gillespie, W.H. and Pfefferkorn, H.W., 1979. Distribution of commonly occurring plant megafossils in the proposed Pennsylvanian system stratotype. In: K.J. Englund, H.H. Arndt and T.W. Henry (Editors), Proposed Pennsylvani. an System Stratotype Virginia and West Virginia (Am. Geol. Inst. Guidebook Ser., 1), 138 pp.

Glukhova, L.V., 1976. On the systematics of the genus Rufloria. Paleontol. Zh., 1976(2): 116-121 (in Russian).

Glukhova, L.V., 1978. On the systematics of cordaitalean leaves (without dorsal grooves). Paleontol. Zh., 1978(4): $115-121$ (in Russian).

Glukhova, L.V. and Men'shikova, L.V., 1980. Cordaitalean microstructures from Late Permian sediments in the Kuznetzk Basin. Paleontol. Zh., 1980(3): 107-117 (in Russian).

Goman'kov, A.V. and Meien, S.V., 1979. On representa. tives of the family Peltaspermaceae from Permian deposits of the Russian platform. Paleontol. Zh., 1979(2): 124138 (in Russian).

Goman'kov, A. V. and Meien, S.V., 1980. On the correlation of macro- and micro-plantfossil complexes in the Permian of Angarida. Paleontol. Zh., 1980(4): 114-122 (in Russian).

Gordon-Gray, K.D., Van Dijk, D.E. and Lacey, W.S., 1976. Preliminary report on equisetalean plants from Lidgetton, Natal. Palaeontol. Afr., 19;43-57.

Gould, R.E., 1976. The succession of Australian pre. Tertiary megafossil floras. Bot. Rev., 41(4): 453-483.

Gould, R.E. and Delevoryas, T., 1977. The biology of Glossopteris: evidence from petrified seed-bearing and pollen-bearing organs. Alcheringa, 1(3/4): 387-399.

Gould, R. and Shibaoka, M., 1980. Some aspects of the formation and petrographic features of coal members in Australia, with special reference to the Tasman Orogenic Zone. Coal Geol. Aust., 2(1): $1 \cdots 29$.

Grauvogel-Stamm, L., 1978. La flore du Grès à Voltzia (Buntsandstein supérieur) des Vosges du Nord (France). Morphologie, anatomie, interprétations phylogénique et paléogéographie. Mém. Sci. Géol., 50: 1-225 (with English and German summaries).

Grzimek, B., 1976. Grzimek's Encyclopedia of Evolution. Van Nostrand and Reinhold, New York, N.Y., 560 pp.

Guerra, M., 1976. Vertebraria (Royle) Schopf 1965, un genre présent dans la Formation Irati du Rio Grande do Sul (Brésil). C.R. 101ème Congr. Natl. Soc. Savantes. Sect. Sci., 1: 51-68.

Guerra, M., 1977. Comentario sobre a estrutura de Vertebraria indica (Royle)Schopf 1965. Bol. Asoc. Latinoamer. Paleobot. Palinol., 4: $2-7$ (with English summary).

Guerra, M., 1978. Uma nova madeira petrificada na 
Formação Irati do Rio Grande do Sul: Ameghiniana, 13(3/4): 254-267 (with English summary).

Guerra-Sommer, M., 1977. Damudoxylon (Maheshwari) Maheshwari, 1972, um género ocorrente no Gondwana do Brasil. Pesqui. Inst. Geocienc. U.F.R.G.S., Porto Allegre, 7: 131-144 (with English summary).

Guerra-Sommer, M., 1978. Ocorrência de género nórdico conífera no Gondwana sul brasileiro. Pesqui. Inst. Geocienc. U.F.R.G.S., Porto Allegre, 10: 65-76 (with English summary).

Guerra-Sommer, M., 1980. Contribução ao estudo das Coníferas do Gondwana brasileiro. Pesqui. Inst. Geocienc. U.F.R.G.S., Porto Allegre, 13: 128-135 (with English summary).

Guerra-Sommer, M., Cazzulo-Klepzig, M. and MarquesToigo, M., 1980. Revisáo fitoestratigráfica do Grupo Itararé no Rio Grande do Sul.3. Área de Faxinal, Município de Guaíba, Rio Grande do Sul. Bol. Inst. Geocienc. Univ. São Paulo, 11: 76-85 (with English summary).

Harris, T.M., 1976. The Mesozoic gymnosperms. Rev. Palaeobot. Palynol., 21(1): 119-134.

Haubold, H., 1980. Zur Gattung Callipteris Brongniart. 2. Indizien für nordamerikanische Callipteriden - Tinsleya, Yakia und Supaia - im Rotliegenden des Thüringer Waldes und der Innersudetischen Mulde. Z. Geol. Wiss., 8(6): 747-763 (with Russian and English summaries).

He, Y., Wu, X., Wu, X., Li, P., Li, H. and Guo, S., 1979. Plants. In: Nanjing Inst. Geol. Palaeontol., Acad. Sin., Qinghai Inst. Geosci. (Editor), Palaeontological Atlas of Northwest China. Qinghai Vol.2. Geol. Publ. House, Beijing, pp.129-167 (in Chinese).

Herbst, R., 1978 Asterotheca golondrinensis n.sp. from the Lower Permian Golondrina Series of the Bajo de la Leona, Santa Cruz, Argentina. Palaeobotanist, 25: 126-130.

Heron, H., 1979. Osmundacaulis stumkei: a memorial to a dedicated collecting team. S. Afr. Lapidary Mag., 13(1): $2-4$.

Holmes, W.B.K., 1977. A pinnate leaf with reticulate venation from the Permian of New South Wales. Proc. Linn. Soc. N.S.W., 102(2): 52-57.

Hsü, J., 1976. On the discovery of a Glossopteris flora in southern Xizang, China and its significance in geology and paleogeography. Sci. Geol. Sin., 1976(4): 323-331 (in Chinese with English summary).

Hsü, J., 1978. On the palaeobotanical evidence for continental drift and Himalayan uplift. Palaeobotanist, 25: 131-145.

Hu, Y., 1980. The feature of fossil plants from the Lower Cangfanggou Group of Upper Permian, northern Xinjiang. Acta Bot. Sin., 22(1): 80-86 (in Chinese with English summary).

Huang, B., 1976. Plants. In: Fossils of North China. Inner Mongolia Vol.1. Palaeozoic. Geol. Publ. House, Beijing, pp.355-379. (in Chinese; edited by Geol. Bur. Inner Mongolia Autonomous Region and NE Inst. Geol. Sci.).

Huang, B., 1977. Permian Flora from the Southeastern Part of the Xiao Hinggan Lin (Lesser Khingan Mt.), NE
China. Geol. Publ. House, Beijing, 79 pp. (in Chinese). Huang, B., 1980. The Plant Kingdom. In: Shenyang Inst. Geol. Miner. Resour. (Editor), Palaeontological Atlas of Northeast China.1. Palaeozoic. Geol. Publ. House, Beijing, pp.526-573 (in Chinese).

Jeyasingh, D.E.P., 1979. A new pattern of cuticular structure in glossopterid leaves. Curr. Sci., India, 48(12): 538.

Jung, W., 1977. Oberpermische Koniferenreste aus dem Sandsteinsockel des Schlernmassivs (Südtiroler Dolomiten). Mitt. Bayer. Staatssamml. Paläontol. Hist. Geol., 17: 235-247 (with English summary).

Kämpfe, L. (Editor), 1980. Evolution und Stammesgeschichte der Organismen. Gustav Fischer, Stuttgart, $411 \mathrm{pp}$.

Kapoor, H.M., 1979. Gondwana of Kashmir - a reap praisal. Proc. 4th Int. Gondwana Symp., Calcutta, 1977, 2: $443-462$.

Keng, H., 1980. A new interpretation of the compressed strobilar structures of Cordaites and conifers. Reinwardtia, 9(4): 377-384.

Kovács-Endrödy, E., 1976. Notes on some Glossopteris species from Hammanskraal (Transvaal). Palaeontol. Afr., 19: 67-95.

Kovács-Endrödy, E., 1977a. The taxonomic status of the genus Rubidgea. Bothalia, 12(2): 313-317.

Kovács-Endrödy, E., 1977b. The systematic position of the genus Gangamopteris McCoy. Bull. Geol. Surv. S. Afr., 60: 19-71.

Kovács-Endrödy, 1979. A re-evaluation of the venation structure of Glossopteris. Ann. Geol. Opname, S. Afr., 12: 107-141.

Krumbiegel, G. and Walther, H., 1977. Fossilien. Sammeln, Präparieren, Bestimmen, Auswerten. Enke, Stuttgart, $336 \mathrm{pp}$.

Lacey, W.S., 1979. Bryophyta. In: R.W. Fairbridge and D. Jablonski (Editors), The Encyclopedia of Paleontology (Encyclopedia of Earth Sciences, 7). Dowden, Hutchinson and Ross, Stroudsburg, Pa., pp.141-148.

Leguizamón, R., 1979. Euryphyllum whittianum Feistman. tel, nuevo integrante de la tafoflora Permico de Baja de Veliz, Prov. San Luis. Bol. Asoc. Latinoamer. Paleobot. Palinol., 6: 5-9 (with English summary)

Lejal-Nicol., A. and Bernardes de Oliveira, M.E.C., 1979. Sur une nouvelle espèce de Cyclodendron Kräusel 1928 du Permien inférieur de l'état de Santa Catarina au Brésil. C.R. 104ème Congr. Natl. Soc. Savantes, 1: 121-132.

Lele, K.M., 1976a. Late Palaeozoic and Triassic floras of India and their relation to the floras of northern and southern hemisphere. Palaeobotanist, 23(2): 89-115.

Lele, K.M., 1976b. Palaeoclimatic implications of Gondwana floras. Geophytology, 6(2): 207-229.

Lemoigne, Y. and Brown, J.T., 1980a. Sur une flore à Glossopteropsida et Lycopsida de Namibie (Sud-Ouest africain). Géobios, 13(4): 541-553 (with English summary).

Lemoigne, Y. and Brown, J.T., 1980b. Révision du genre Lycopodiopsis B. Renault 1890. Géobios, 13(4): 555-577 (with English summary). 
Le Roux, S.F., 1976. On some "northern" elements in the Lower Gondwana flora of Vereeniging, Transvaal. Palaeontol. Afr., 19: 59 65.

Lichter. G., 1979. Fossilien bergen, präparieren und ausstellen. Geräte und Techniken unter besonderer Berücksichtigung der Gesteinsarten. Ein Praktikum für den Fossilienfreund und -sammler. Franckh'sche Verlagshandlung, Stuttgart, $144 \mathrm{pp}$.

Lichter, G., 1980. Fossielen verzamelen, prepareren en tentoonstellen. Thieme, Zutphen, $144 \mathrm{pp}$.

Maithy, P.K.,.1977a. A new species of Zalesskioxylon from the Glossopteris bearing beds of Zambesi Basin, Mozambique. Geophytology, 7(1): 24-28.

Maithy. P.K., 1977b. Three new fern fronds from the Glossopteris flora of India. Palaeobotanist, 24(2): 96-101

Maithy, P.K., 1977c. Revision of some fossil plants from the Karharbari Formation, Giridih Coalfield, Bihar. Palaeobotanist, 23(3): 220222 .

Maithy, P.K., 1978. Further observations on Indian Lower Gondwana Sphenophyllales. Palaeobotanist, 25 $266 \cdot 278$.

Maithy, P.K. and Mandal, J., 1978. Further observations on Stellotheca robusta Surange and Prakash. Palaeobotanist. 25: 279289 .

Major, A., 1979. Collecting Fossils. Bartholomew, Edinburgh, $124 \mathrm{pp}$.

Mamay, S.H., 1976. Paleozoic origin of the cycads. Prof. Pap. Geol. Surv. U.S.A., 934: 1-48.

Mamay, S.H., 1978. Vojnovskyales in the Lower Permian of North America. Palaeobotanist, 25: 290-297.

Mapes, G. and Schabilion J.T., 1979. Millaya gen.n., an Upper Paleozoic genus of Marattialean synangia. Am. J. Bot., 66(10): 1164-1.172.

Marguerier, J., 1976. Paléoxylologie du Karroo malgache étude d'un bois fossile de la Sakamena (District de Mahabo) Dadoxylon (Araucarioxylon) malaimbandense n.sp. C.R. 97 Congr. Natl. Soc. Savantes, Sect. Sci.: 87-105.

Marguerier, J., 1977. Sur la ponctuation simple dans le champs de croisement de quelques structures paléozoï ques et mesozoïques. C.R. 102ème Congr. Natl. Soc Savantes, Sect. Sci., 1: 79-97.

Marguerier, J. and Pacaud, G., 1980. La 3e zone de bois silicifiés de l'Autunien du bassin d'Autun (France): Nouvelles données sur un gisement de bois silicifiés. Caractères paléobotaniques. Bull. Soc. Hist. Nat. Autun. 95: 4160

Meien, S.V., 1977. Cardiolepidaceae -- new Permian conifer family from northern Eurasia. Paleontol. Zh., 1977(3): 128-138 (in Russian).

Meien, S.V., 1978. Permian conifers of West Angaraland and new puzzles in the coniferalean phylogeny. Palaeobotanist, 25: 298-313.

Meien, S.V., 1979. Permian predecessors of the Mesozoic pteridosperms in western Angaraland, U.S.S.R. Rev. Palaeobot. Palynol., 28(2): 191-201.

Meien, S.V. and Goman'kov, A.V., 1980. The peltaspermous pteridosperms of the genus Tatarina. Paleontol. $\mathrm{Zh}$., 1980(2): 116 132 (in Russian).

Mildenhall, D.C., 1976. Glossopteris ampla Dana from New
Zealand Permian sediments. New Zealand J. Geol. Geophys., 19(1): 130-132

Millan, J.H.. 1977. Sementes platispérmicas do Eogondwana de Cerquilho, São Paulo (Bacia do Paraná). An. Acad. Bras. Cienc., 49(4): $581-595$ (with English summary).

Millan, J.H. and Dolianiti, E., 1979. Esfenófitas do Eogondwana de Cerquilho São Paulo (Bacia do Paraná).3. Gênero Phyllotheca. Bol. Asoc. Latinoamer. Paleobot. Palinol., 6: 1 4 (with English summary).

Millan, J.H. and Dolianiti, E., 1980a. Sobre um novo "Fertiliger" no Eogondwâna de Cerquilho, São Paulo (Bacia de Paraná), Brasil. In: Actas 2 Congr. Argent. Paleontol. Bioestratigr. y 1 Congr. Latinoamer. Paleontol., Buenos Aires, 1978, 4. Simp. Carbónico-Pérmico, 103111 (with English summary).

Millan, J.H. and Dolianiti, E., 1980b. Esfenófitas do Eogondwana de Cerquilho, São Paulo (Bacia de Paraná). Brasil. 2. Género Stephanophyllites, gen. nov. In: Actas 2 Congr. Argent. Paleontol. Bioestratigr. y 1 Congr. Latinoamer. Paleontol., Buenos Aires, 1978, 4. Simp. Carbónico-Pérmico, pp.113-127 (with English summary).

Millay, M.A. and Taylor, T.N., 1979. Paleozoic seed fern pollen organs. Bot. Rev., 45(3): 301-375 (with French, German, Spanish and Russian summaries).

Miller, C.N., 1978. Mesozoic conifers. Bot. Rev., 43(2): 217280

Minato, M., 1977. Carboniferous-Permian plant remains found at the border of Lake Panguipulli, Valdivia Province, Chile, with a description of the flora by Dr. T. Tanai. In: T. Ishikawa and L. Arguirre (Editors). Comparative studies on the Geology of the Circum. Pacific orogenic Belt in Japan and Chile. First report. Jpn. Soc. Promot. Sci., Tokyo, pp.69-79 (with Spanish summary).

Mundlos, R., Adam, K.D., Bloos, G., Dietl, G., Urlichs, M. Warth, M., Wild, R. and Ziegler, B., 1976. Wunderwelt in Stein. Fossilfunde - Zeugen der Urzeit. Bertelsmann, Gütersloh, $280 \mathrm{pp}$

Mussa, D., 1976. Paleoxiloanatomia Brasileiro. 2. Novo gênero de lenho fóssil da Formaçào Irati, Estado de Sào Paulo. An. Acad. Bras. Cienc., 46(3/4): 617 634 (with English summary)

Mussa, D., 1978a. Estruturas vasculares iniciais em lignospécimes Gondwanicos interpretadas à luz das pesquisas ontogenéticas modernas. Bol. Inst. Geocienc. Univ. São Paulo, 9: 95 104 (with English summary).

Mussa, D., 1978b. Brasilestiloxylon e Solenobrasilioxylon, dois novos géneros Gondwànicos na Formação Irati, Estado de Sào Paulo, Brasil. Bol. Inst. Geocienc. Univ. São Paulo, 9: 118-127 (with English summary).

Mussa, D., 1978c. On the anatomy of wood showing affinities with the genus Vertebraria Royle, from the Irati Formation, State of São Paulo, Brazil. Bol. Inst. Geocienc. Univ. São Paulo, 9: 153-201 (with English summary).

Mussa, D., 1980. Ocorréncia do genero Antarticoxylon Seward, na Formação Irati (Permiano) do Estado de São Paulo, Brasil. In: Actas 2 Congr. Argent. Paleontol. 
Bioestratigr. y 1. Congr. Latinoamer. Paleontol., Buenos Aires, 1978, 4. Simp. Carbónico-Pérmico, pp.139-155 (with English summary).

Mussa, D., De Carvalho, R.G. and Dos Santos, P.R., 1980. Estudio estratigráfico e paleoecológico em ocorréncias fossilíferas da Formaçao Iratí, Estado de São Paulo, Brasil. Bol. Inst. Geocienc. Univ. São Paulo, 11: 142-149.

Mussa, D., De Carvalho, R.G. and Saad, A.R., 1978. Medula do tipo Artisia na Formação Iratí (Permiano), Estado de São Paulo, Brasil. Bol. Inst. Geocienc. Univ. São Paulo, 9: 114-117 (with English summary).

Niklas, K.J., 1977. Ontogenetic constructions of some fossil plants. Rev. Palaeobot. Palynol., 23(5): 337-357.

Paliwal, G.S., Gary, M. and Harjal, N., 1976. Evolution of foliar venation in embryobionta. Curr. Sci., India, 45(5): 171-174.

Pant, D.D., 1977. The plant of Glossopteris. J. Indian Bot. Soc., 56(1): 1-23.

Pant, D.D. and Choudhury, A., 1977. On the genus Belemnopteris Feistmantel. Palaeontographica B, 164(5/6): 153-166

Pant, D.D. and Misra, L., 1976. Compressions of a new type of pteridophyll, Asansolia gen. nov. from the Lower Gondwanas of the Raniganj Coalfield, India. Palaeontographica B, 155(5/6): 129-139.

Pant, D.D. and Misra, L., 1977. On two genera of pteridophylls: Damudosorus gen. nov, and Trithecopteris gen. nov, from the Lower Gondwanas of the Raniganj coalfield. Palaeontographica B, 164(1/3): 76-86.

Pant, D.D. and Singh, S., 1978. Cuticular structure and affinities of Cheirophyllum lacerata (Feistmantel) n.comb. Palaeobotanist, 25: 353-362.

Pant, D.D. and Singh, S., 1979. Caulophyllites indica gen. et sp.nov. from the Giridih coalfield, India. Palaeontographica $\mathrm{B}, 169(4 / 6)$ : 107-115.

Pawlowska, K., 1978. Characteristics of the Permian of the Promnik Basin based on data from the Ruda Strawczyn. ska borehole. Biul. Inst. Geol., 309(13): 65-98.

Pfefferkorn, H.W. and Resnik, D.A., 1980. The occurrence of Sphenopteris germanica (Pteridosperm, Autunian, Lower Permian) in North America. J. Paleontol., 54(1): 81- 84 .

Prasad, M.N.V. and Chandra, S., 1978a. First record of Taxopitys from the Indian Lower Gondwana. Curr. Sci. India, 47(10): 347-348.

Prasad, M.N.V. and Chandra, S., 1978b. Australoxylon from the Kamthi Beds of Lower Gondwana, India. Curr. Sci. India, 47(16): 597.

Prasad, M.N.V. and Chandra, S., 1980. Palaeospiroxylon, a new gymnospermous wood from Raniganj coalfield, India. Palaeobotanist, 26(3): 230-236 (with Hindi summary).

Quenstedt, F.A., 1885. Atlas zum Handbuch der Petrefaktenkunde. Geschiebesammler, 13(1): 39-63. (facsimile of plates; 3rd. ed., 1979).

Ratkevich, R. and La Fon, N., 1978. Field guide to New Mexico fossils. Dinograph Southwest, Alamogordo, N. Mex., $78 \mathrm{pp}$.

Remy, W., 1978. Die Sphenopteris germanica-Gruppe in den "Süplinger Schichten", Flechtinger Höhenzug. Ein Be- leg für das Autun-Alter. Argumenta Palaeobot., 5: 161-165 (with English summary).

Remy, W. and Remy, R., 1977. Die Floren des Erdaltertums. Glückauf, Essen, 468 pp.

Remy, W. and Remy, R., 1978a. Beiträge zur Flora des Stefans und Autuns. Argumenta Palaeobot., 5: 195-204 (with English summary).

Remy, W. and Remy, R., 1978b. Calamitopsis n.gen. und die Nomenklatur und Taxonomie von Calamites Brongniart, 1828. Argumenta Palaeobot., 5: 1-10 (with English summary).

Remy, W. and Remy, R., 1978c. Die Flora des Perms im Trompia-Tal, und die Grenze Saxon/Thuring in den Alpen. Argumenta Palaeobot., 5: 57 90 (with English summary).

Remy, W. and Remy, R., Leisman, G.A. and Hass, H., 1980 Der Nachweis von Callipteris flabellifera (Weiss 1879) Zeiller 1898 in Kansas, U.S.A. Argumenta Palaeobot., 6: 1-36 (with English summary).

Retallack, G.J., 1977. Reconstructing Triassic vegetation of eastern Australasia: a new approach for the biostratigraphy of Gondwanaland. Alcheringa, 1(3/4): 247-277.

Retallack, G., 1980. Late Carboniferous to Triassic megafossil floras from the Sydney Basin. In: Guide Sydney Basin: Geol. Surv. N.S.W. Dep. Mines Miner. Resour., 26: $384-430$.

Richter, A.E., 1979. Südfrankreich und seine Fossilien. Geologie und Paläontologie von Causses und Provence. Kosmos, Stuttgart, $192 \mathrm{pp}$.

Rigby, J.F., 1978a. Permian glossopterid and other cycadopsid fructifications from Queensland. Publ. Geol. Surv. Qld., 367: 1-21.

Rigby, J.F., 1978b. The Permian plant Belemnopteris in Queensland. Publ. Geol. Surv. Qld., 367: 25-27.

Rigby, J.F., Maheshwari, H.K. and Schopf, J.M., 1980. Revision of Permian plants collected by J.D. Dana during 1839-1840 in Australia. Publ. Geol. Surv. Qld., 376: $25 \mathrm{pp}$.

Rösler, O., 1978. Novas ocorréncias na Formação Rio do Rasto, Permiano Superior, Estado do Paraná. Bol. Inst. Geocienc. Univ. São Paulo, 9: 127-132 (with English summary).

Rösler, O., 1979. Plantas fosseis de São João de Triunfo (PR), Formação Rio Bonito e suas implicações geológicas. Actas 2 Simp. Reg. Geol., Río Claro, 1979, 1: 181-194 (with English summary).

Rothe, H.W., 1976. Kleine Versteinerungskunde. Hallwag, Bern, 5 ed., 64 pp.

Rumpel, D., 1980. Ein Myeloxylon-Fund aus ErlangenDechsendorf. Geol. B1. Nordost Bayern, 30(3/4): 224-225.

Sal'menova, K.Z., 1978. Permian flora from northern Pribalkhas'ya. Paleontol. Zh., 1978(4): 122-127 (in Russian).

Sal'menova, K.Z., 1979. Characteristics of Permian flora of the southern Kazakh S.S.S.R., U.S.S.R., and its relationships with neighbouring floras. Paleontol., Zh., 1979(4): 119-127 (in Russian)

Sarkar, S.S., 1978. Gondwana link between India and Australia evidenced by Glossopteris correlation. Pac Geol., Jpn., 13: 77-86. 
Schaumberg, G., 1977. Der Richelsdorfer Kupferschiefer und seine Fossilien. 4. Die pflanzlichen Fossilien des Kupferschiefers, sonstige Lebensspuren, Aufrechnung der Fundhäufigkeit. Aufschluss, 28(11): 427-442.

Scheven, J., 1979. Daten zur Evolutionslehre im Biolo gieunterricht. Hänssler, Neuhausen-Stuttgart, $132 \mathrm{pp}$.

Schniepp, H., 1979. Leitfossilien des Perm. Kosmos, 75(10): 787.794.

Schopf. J.M., 1976. Morphologic interpretation of fertile structures in glossopterid gymnosperms. Rev. Palaeobot. Palynol., 21(1): 2564.

Schweitzer, H.-J., 1977. Die raeto-jurassischen Floren des Iran und Afghanistans. 4. Die raetische Zwitterblüte Irania hermaphroditica nov. spec. und ihre Bedeutung für die Phylogenie der Angiospermen. Palaeontographica B, 161(1/4): 98 145 (with English summary).

Schweitzer, H.-J., 1978. The derivation of the hermaphroditic flower of the angiosperms by consideration of new fossils from the Rhaetic of Iran. Cour. Forsch. -Inst. Senckenberg, 30: 16 $\cdots 26$.

Sopeña, A., Virgili, C., Doubinger, J. and Adloff, M.C., 1977. El Permico y el Triasico del borde sur-este del Sistema Central. Cuad. Geol. Ibér., 4: 623 648.

Srivastava, A.K., 1977a. Palaeobotanical evidence for the presence of Karharbari Stage in the Auranga coalfield, Bihar: Megaflora. Palaeobotanist. 23(3): 206 219.

Srivastava, A.K., 1977b. Studies in the Glossopteris flora of India. 42. Barakar plant megafossils and miospores fromAuranga coalfield, Bihar. Palaeobotanist, 24(1): 5069 .

Srivastava, A.K., 1978. Studies in the Glossopteris flora of India. 43. Some new plant fossils from the Lower Gondwana sediments of Auranga coalfield, Bihar. Palaeobotanist, 25: 486 495 .

Srivastava, A.K., 1979. Studies in the Glossopteris flora of India. 44. Raniganj plant megafossils and miospores from Auranga coalfield, Bihar. Palaeobotanist, 26(1): 72 94

Stewart. W.N., 1976. Polystely, primary xylem and the Pteropsida. In: Twenty-first Sir Albert Charles Seward Memorial Lecture. Birbal Sahni Inst. Palaeobot. Palynol., Lucknow, 13 pp.

Steel, R. and Harvey, A.P., 1979. The Encyclopaedia of Prehistoric Life. Mitchell Beazley, London, $380 \mathrm{pp}$.

Storch, D., 1980. Sphenophyllum-Arten aus drei intramontanen Karbonbecken -- pflanzengeographische Besonderheiten im mitteleuropäischen Karbon. Schriftenr. Geol. Wiss., 16: 171-273 (with French, English and Russian summaries)

Storch, D. and Barthel, M., 1980. Adventivwurzeln bei Sphenophyllum-Arten. Z. Geol. Wiss., 8(12): 1571-1579.

Strasburger, E., 1978. Lehrbuch der Botanik für Hochschulen. Fischer, Stuttgart, 31 Ed., 1078 pp.

Surange, K.R. and Chandra, S., 1978. Morphology and affinities of Glossopteris. Palaeobotanist, 25: 509-524.

Taylor, T.N. and Millay, M.A., 1979. Pollination biology and reproduction in early seed plants. Rev. Palaeobot. Palynol., 27(3/4): $329 \cdot 355$

Teixeira, C. and Pais, J., 1976. Introdução à Paleobotanica. As grandes Fases da Evolução dos Vegetais. Livraria escolar, Lisboa, $210 \mathrm{pp}$.
Tenchov, Y.G., 1977a. Fossil flora from the Stephanian Permian outcrops in northwestern Bulgaria. 3. Sphenopteris. Neuropteridaceae, Taeniopteris. Paleontol. Stratigr. Litol, 6(2): $15 \cdot 26$ (in Bulgarian with Russian and English summaries).

Tenchov, Y.G., 1977b. Fossil flora from the Stephanian Permian outcrops in northwestern Bulgaria. 4. Alethopteridacea, Callipteris, Odontopteris, Gymnospermophyta, seminae. Paleontol. Stratigr. Litol., 7: 3-14 (in Bulgarian with Russian and English summaries).

Tian, B. and Zhang, L., 1980. Fossil Atlas of Wangjiazhai Mine Region in Shuicheng, Guizhou Province. Publ. House Coal Ind., Beijing, 98 pp. (in Chinese).

Van Amerom, H.W.J., Boersma, M., Niedermayr, G. and Scheriau-Niedermayr, E., 1976. Das permische Alter der "Karbon"-Flora von Kötschach (Kärnten, Österreich). Carinthia II, 86: 93-101 (with English summary).

Van Dijk, D.E., Gordon-Gray, K.D. and Lacey, W.S., 1978. SEM studies of Karoo fossils. Proc. Electron Microsc. Soc. S. Afr.. 8: 119-120.

Van Dijk, D.E., Gordon-Gray, K.D., Reid, C. and Lacey, W.S., 1979. Contribution to knowledge of the Lower Beaufort (Upper Permian) Flora of Natal, South Africa. Proc. 4th Int. Gondwana Symp., Calcutta, 1977. 1: 109121

Verbitskaya, N.G., 1980a. New Permian liverworts from Siberia. In: I.N. Srebrodol'skaya, G.A. Stukalina, A.F. Abushik and N.N. Bobkova (Editors), New species of ancient plants and invertebrates in the U.S.S.R.: Plants. Tr. Vses. Nauchno-Issled. Geol. Inst. Leningrad, 204: 2126 (in Russian).

Verbitskaya, N.G., 1980b. New Permian species of Koretrophyllites from Siberia. In: I.N. Srebrodol'skaya, G.A. Stukalina, A.F. Abushik and N.N. Bobkova (Editors), New species of ancient plants and invertebrates in the U.S.S.R.: Plants. Tr. Vses. Nauchno-Issled. Geol. Inst. Leningrad, 204: 29 32 (in Russian).

Verbitskaya, N.G., 1980c. New late Permian articulate plants from Siberia. In: I.N. Srebrodol'skaya, G.A. Stukalina, A.F. Abushik and N.N. Bobkova (Editors), New species of ancient plants and invertebrates in the U.S.S.R.: Plants. Tr. Vses. Nauchno-Issled. Geol. Inst. Leningrad, 204: 36-37 (in Russian).

Verbitskaya, N.G., 1980d. New seeds from the Lower Permian of the Tungusk Basin. In: I.N. Srebrodol'skaya, G.A. Stukalina, A.F. Abushik and N.N. Bobkova (Editors), New species of ancient plants and invertebrates in the U.S.S.R.: Plants. Tr. Vses. Nauchno-Issled. Geol. Inst. Leningrad, 204: 6970 (in Russian).

Vladimirovich, V.P., 1980. New Late Permian ferns from the Priural and the Kuznetsk Basin. In: I.N. Srebrodol'. skaya, G.A. Stukalina, A.F. Abushik and N.N. Bobkova (Editors), New species of ancient plants and invertebrates in the U.S.S.R.: Plants. Tr. Vses. Nauchno-Issled. Geol. Inst. Leningrad, 204: $43-47$ (in Russian).

Vogellehner, D., 1977a. Paläontologie, Grundlagen, Erkenntnisse: Geschichte der Organismen. Herder, Frejburg i/Br., $110 \mathrm{pp}$.

Vogellehner, D., 1977b. Die systematische Stellung der 
Koniferen. In: P. Parey (Editor), Die Fichte, 1: Parey, Hamburg, pp.1-8.

Vozenin-Serra, C., 1978. Sur un nouveau gère de gymnosperme du Permien supérieur cambodgien: Cathaysioxylon khmerinum (Serra 1966) Vozenin-Serra, nov.nom. C.R. 103ème Congr. Natl. Soc. Savantes, Paléobot., 2: $53-63$.

Vozenin-Serra, C., 1979. Etude de quelques empreintes de végétaux fossiles du Paléozoïque supérieur du Laos. C.R. 104ème Congr. Natl. Soc. Savantes, 1: 155-174.

Wang, R., 1979. On the occurrence of a bipinnate frond of Neuropteridium ? nervosum Halle from the Upper Shihhotse Formation of northern Jiangsu. Acta Palaeontol. Sin., 18(2): 202-203 (in Chinese with English summary).

Waterhouse, J.B., 1978. Late Paleozoic. In: R.O. Suggate and G.R. Stevens (Editors), The Geology of New Zealand; the Fossil Record and Paleogeography; Interpretation of the Fossil Record. Publ. New Zealand Geol. Surv., 2: 706- 710 .

Wesley, A., 1977. Pterophytes, Cycadophytes, Coniferophytes, Angiosperms. In: A. Hallam (Editor), Planet Earth - an Encyclopedia of Geology. Elsevier-Phaidon, Oxford, pp.251-253.

White, M.E., 1978. Reproductive structures of the Glossopteridales in the plant fossil collection of the Australian museum. Rec. Aust. Mus., 31(10/12): 473-505.

$\mathrm{X}$, 1980. Réconstruction d'un paysage de l'Autunien d'Autun (France). Minéraux et Fossiles, Meung-surLoire, 14 pp. (with French, English and German text).

Yuasa, Kadzuo, 1979. The evolution of gymnosperms and palaeontology. Iden (Heredity), 33(6): 38-45 (in Japanese).
Zhang, J., 1978. Plants. In: Fossil Atlas of Southwest China. Guizhou Vol.2. Geol. Publ. House, Beijing, pp.458-491 (in Chinese; edited by Stratigr. Geol. Working Team Guizhou Prov.)

Zhang, S., 1980. Note on the ecological significance of some Cathaysian floral elements. In: Pap. First Conf. IOP, London, Reading, 1980. Nanjing Inst. Geol. Palaeontol. Acad. Sinica, $6 \mathrm{pp}$.

Zhang, S. and Mo. Z., 1979. New forms of seed-bearing fronds from the Cathaysia flora in Henan, China. Pap. 9 Int. Congr. Carboniferous Stratigr. Geol., Nanjing Inst. Geol. Palaeontol. Acad. Sinica, 5 pp.

Zhao, X., Mo, Z., Zhang, S. and Yao, Z., 1980. Late Permian flora from W. Guizhou and E. Yunnan. In: Stratigraphy and Palaeontology of Upper Permian Coal Measures in W. Guizhou and E. Yunnan. Sci. Press. Beijing; pp.70-122 (in Chinese; edited by Nanjing Inst. Geol. Palaeontol. Acad. Sinica)

Zimina, V.G., 1976a. Relict plants in the Late Permian flora of Primor'ye. Ocherki Geol. Paleontol. Dal'nego Vostoka, Vladivostok: 30-33 (in Russian).

Zimina, V.G., 1976b. Additional material to the Permian cordaites from Primor'ye. Ocherki Geol. Paleontol. Dal'nego Vostoka, Vladivostok: 34-38 (in Russian).

Zimina, V.G., 1977. The Flora in the Early and Early Late Permian of southern Primor'ye. Nauka, Moscow, 127 pp.

Zimina, V.G., 1978. Systematic composition of the Early Permian flora of southern Primor'ye and some questions regarding the morphology of cordaitalean leaves. In: A.G. Ablaev, B.V. Poyarkov and Z.N. Poyarkova (Editors), Biostratigraphy of the southern Far East (Phanerozoic). Akad. Nauk Dal'nevost. Nauchn. Tsentr., Vladivostok, pp.36-45 (in Russian). 